\title{
Direct Catalytic Chemoselective $\alpha$-Amination of Acylpyrazoles: A Concise Route to Unnatural $\alpha$-Amino Acid Derivatives
}

\author{
Keisuke Tokumasu, Ryo Yazaki* and Takashi Ohshima* \\ Graduate School of Pharmaceutical Sciences, Kyushu University, Fukuoka, Japan \\ ohshima@phar.kyushu-u.ac.jp, yazaki@phar.kyushu-u.ac.jp
}

\author{
1. General \\ 2. Instrumentation \\ 3. Materials \\ 4. Substrate Syntheses and Characterization \\ 5. General Procedure and Characterization of the Products \\ 6. Transformation of the Product \\ 7. References \\ 8. NMR Spectra of New Compounds
}

\section{General}

All reactions were carried out using heat gun dried glassware under a positive pressure of dry argon unless otherwise noted. The test tubes were fitted with a 3-way glass stopcock and catalytic reactions were run under argon atmosphere. Air- and moisture-sensitive liquids were transferred via a syringe and a stainless-steel needle. Reactions were magnetically stirred and monitored by thin layer chromatography using Merck Silica Gel 60 F254 plates. All work-up and purification procedures were carried out with reagent-grade solvents under ambient atmosphere. Flash chromatography was 
performed using silica gel 60N (spherical neutral, particle size 40-50 $\mu \mathrm{m}$ ) purchased from Kanto Chemical Co. Ltd.

\section{Instrumentation}

NMR was recorded on $500 \mathrm{MHz}$ Bruker Advanced III. Chemical shifts for proton are reported in parts per million downfield from tetramethylsilane and are referenced to residual protium in the NMR solvent $\left(\mathrm{CDCl}_{3}: \delta 7.26 \mathrm{ppm}\right)$. For ${ }^{13} \mathrm{C} \mathrm{NMR}$, chemical shifts were reported in the scale relative to NMR solvent $\left(\mathrm{CDCl}_{3}: 77.0 \mathrm{ppm}\right)$ as an internal reference. NMR data are reported as follows: chemical shifts, multiplicity (s: singlet, d: doublet, dd: doublet of doublets, t: triplet, q: quartet, sep: septet, m: multiplet, br: broad signal), coupling constant $(\mathrm{Hz})$, and integration. Infrared (IR) spectra were recorded on with Shimadzu FTIR-8400. High-resolution mass spectroscopy (HRMS) was obtained with Waters ACQUITY UPLC $^{\circledR}-$ LCT-Premier $^{\text {TM }}$ XE system and Bruker MicrOTOF II.

\section{Materials}

DMF was dried over MS4A. Cu(OTf $)_{2}$ and 3,5-dimethylpyraole and commercially available carboxylic acids were purchased from $\mathrm{TCl}$ and used as received. $\mathrm{Cu}(\mathrm{OTf})_{2}$ was stored in a dry box. Dichloromethane and THF were purchased from Wako Pure Chemical Industries, Ltd. (Deoxidized) and dried over MA 4A. Molecular Sieves 4A was purchased from Nacalai Tesque, Inc. Magnesium powder was purchased from Aldrich and stored in in a dry box. Iminoiodinane was prepared by following reported procedure. ${ }^{1}$

\section{Substrate Syntheses and Characterization}

General Procedure for the synthesis acylpyrazole (acid chloride method) (1a-1g) ${ }^{2}$ : To a solution of 3,5dimethylpyrazole (1.0 equiv) and triethylamine (2.0 equiv) in dry toluene $(1.0-0.2 \mathrm{M})$ was added corresponding acid chloride (1.1 equiv) at $0{ }^{\circ} \mathrm{C}$ under argon atmosphere. The suspended reaction mixture was stirred under reflux conditions (oil bath $120^{\circ} \mathrm{C}$ ) for $1 \mathrm{~h}$. After the reaction mixture was cooled to room temperature, the crude mixture was filtered through silica short column and washed with toluene. After evaporation of organic solvent under reduced pressure, the crude mixture was purified by distillation or silica gel column chromatography to give the desired acylpyrazole 1. 


\section{General Procedure for the synthesis acylpyrazole (EDC/HOBt method) (1h-1ס):}

The round bottom flask equipped with a magnetic stirring bar and 3-way glass stopcock was evacuated and filled with argon (three cycles). To the solution of carboxylic acid (1.0 equiv) in dry DMF (1.0 M) was added EDC. $\mathrm{HCl}$ (1.2 equiv), $\mathrm{HOBt} \cdot \mathrm{H}_{2} \mathrm{O}$ (1.2 equiv), 3,5-dimethylpyrazole (1.1 equiv) and $N$ methylmorpholine (2.0 equiv) at $0{ }^{\circ} \mathrm{C}$. After stirring for $24 \mathrm{~h}$ at room temperature, it was quenched by $0.1 \mathrm{M} \mathrm{HCl}$ or $10 \%$ citric acid aq. The resultant mixture was extracted with EtOAc, and combined organic layer was washed with sat. $\mathrm{NaHCO}_{3}$ solution and brined successively. The resulting organic layers were dried over $\mathrm{Na}_{2} \mathrm{SO}_{4}$. After removal of solvent under reduced pressure, the crude mixture was purified by silica gel column chromatography to afford desired acylpyrazole $\mathbf{1}$.

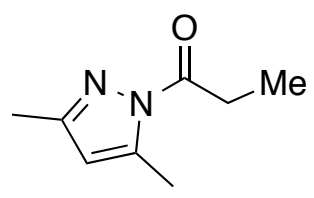

1-(3,5-dimethyl-1H-pyrazol-1-yl)propan-1-one (1a) (colorless oil) (CAS Registry Number 131275-69-9)

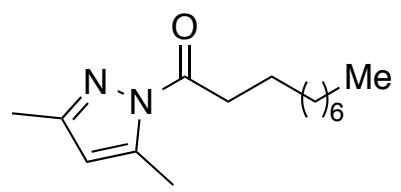

1-(3,5-dimethyl-1H-pyrazol-1-yl)decan-1-one (1b) (colorless oil) ; ${ }^{1} \mathrm{H}-\mathrm{NMR}\left(500 \mathrm{MHz}, \mathrm{CDCl}_{3}\right.$ ) (ppm) $\delta$ $5.94\left(\mathrm{~s}, 1 \mathrm{H}\right.$, pyrazole), $3.09\left(\mathrm{t}, J=7.5 \mathrm{~Hz}, 2 \mathrm{H}, \mathrm{COCH}_{2}\right), 2.54\left(\mathrm{~s}, 3 \mathrm{H}, \mathrm{ArCH}_{3}\right), 2.24\left(\mathrm{~s}, 3 \mathrm{H}, \mathrm{ArCH}_{3}\right), 1.76-1.70$ $(\mathrm{m}, 2 \mathrm{H}), 1.41-1.27(\mathrm{~m}, 12 \mathrm{H}), 0.88\left(\mathrm{t}, J=7.0 \mathrm{~Hz}, 3 \mathrm{H}, \mathrm{CH}_{3}\right) ;{ }^{13} \mathrm{C} \mathrm{NMR}\left(125 \mathrm{MHz}, \mathrm{CDCl}_{3}\right) \delta$ 174.2, 151.6, 143.9, $110.9,35.1,31.8,29.41,29.38,29.3,29.1,24.3,22.7,14.6,14.1,13.8$; IR (thin film, NaCl) 2926, 2854, $1730,1582,1456,1412,1379,1343,1252,961 \mathrm{~cm}^{-1}$; HRMS (ESI, Na) $\mathrm{m} / \mathrm{z}$ calc'd for $\mathrm{C}_{15} \mathrm{H}_{27} \mathrm{~N}_{2} \mathrm{O}(\mathrm{M}+\mathrm{H})^{+}$ 251.2118 , found 251.2123<smiles>Cc1cc(C)n(C(=O)CCC(C)C)n1</smiles>

1-(3,5-dimethyl-1H-pyrazol-1-yl)-4-methylpentan-1-one (1c) (colorless oil); ${ }^{1} \mathrm{H}-\mathrm{NMR}\left(500 \mathrm{MHz}, \mathrm{CDCl}_{3}\right.$ ) 
(ppm) $\delta 5.94\left(\mathrm{~s}, 1 \mathrm{H}\right.$, pyrazole), 3.10, (t, $\left.J=8.0 \mathrm{~Hz}, 2 \mathrm{H}, \mathrm{C}(\mathrm{O}) \mathrm{CH}_{2} \mathrm{CH}_{2}\right), 2.53\left(\mathrm{~s}, 3 \mathrm{H}, \mathrm{ArCH}_{3}\right), 2.24(\mathrm{~s}, 3 \mathrm{H}$, $\left.\left.\mathrm{ArCH}_{3}\right), 1.69-1.61\left(\mathrm{~m}, 3 \mathrm{H}, \mathrm{CH}_{2} \mathrm{CH}\left(\mathrm{CH}_{3}\right)_{2}\right), 0.95\left(\mathrm{~d}, J=6.0 \mathrm{~Hz}, 6 \mathrm{H}, \mathrm{CH}\left(\mathrm{CH}_{3}\right)_{2}\right)\right) ;{ }^{13} \mathrm{C} \mathrm{NMR}\left(125 \mathrm{MHz}, \mathrm{CDCl}_{3}\right) \delta$ 174.5, 151.7, 144.0, 110.9, 33.22, 33.19, 27.7, 22.4, 14.6, 13.8; IR (thin film, NaCl) 2957, 2923, 2870, $1730,1584,1470,1412,1379,1346,1314,963 \mathrm{~cm}^{-1} \mathrm{HRMS}(\mathrm{ESI}, \mathrm{H}) \mathrm{m} / \mathrm{z}$ calc'd for $\mathrm{C}_{11} \mathrm{H}_{19} \mathrm{~N}_{2} \mathrm{O}(\mathrm{M}+\mathrm{H})^{+}$ 194.1492, found 195.1494 .

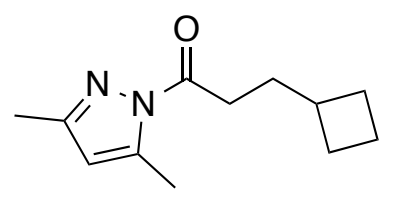

3-cyclobutyl-1-(3,5-dimethyl-1H-pyrazol-1-yl)propan-1-one (1d) (colorless oil); ${ }^{1} \mathrm{H}-\mathrm{NMR}$ (500MHz, $\left.\mathrm{CDCl}_{3}\right)(\mathrm{ppm}) \delta 5.94\left(\mathrm{~s}, 1 \mathrm{H}\right.$, pyrazole), $3.00\left(\mathrm{t}, J=7.5 \mathrm{~Hz}, 2 \mathrm{H}, \mathrm{C}(\mathrm{O}) \mathrm{CH}_{2} \mathrm{CH}_{2}\right), 2.53\left(\mathrm{~s}, 3 \mathrm{H}, \mathrm{ArCH}_{3}\right.$ ), 2.36 (sep, J $\left.=7.5 \mathrm{~Hz}, 1 \mathrm{H}, \mathrm{CH}_{2} \mathrm{CH}\left(\mathrm{CH}_{2}\right) \mathrm{CH}_{2}\right), 2.23\left(\mathrm{~s}, 3 \mathrm{H}, \mathrm{ArCH}_{3}\right), 2.10-2.04(\mathrm{~m}, 2 \mathrm{H}), 1.88-1.79(\mathrm{~m}, 4 \mathrm{H}), 1.68-1.60(\mathrm{~m}$, $2 \mathrm{H}) ;{ }^{13} \mathrm{C} \mathrm{NMR}\left(125 \mathrm{MHz}, \mathrm{CDCl}_{3}\right) \delta 174.2,151.7,144.0,110.9,35.4,33.1,31.5,28.1,18.3,14.6,13.8 ; \mathrm{IR}$ (thin film, NaCl) 2928, 2862, 1732, 1582, 1416, 1377, 1348, 1325, 1248, $961 \mathrm{~cm}^{-1}$ HRMS (ESI, H) $\mathrm{m} / \mathrm{z}$ calc'd for $\mathrm{C}_{12} \mathrm{H}_{19} \mathrm{~N}_{2} \mathrm{O} 207.1492(\mathrm{M}+\mathrm{H})^{+}$, found 207.1496.

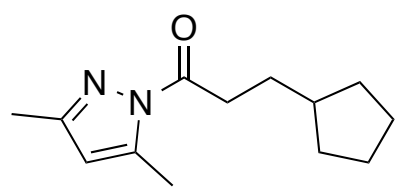

3-cyclopentyl-1-(3,5-dimethyl-1H-pyrazol-1-yl)propan-1-one (1e) (colorless oil); ${ }^{1} \mathrm{H}-\mathrm{NMR}\left(500 \mathrm{MHz}, \mathrm{CDCl}_{3}\right.$ ) (ppm) $\delta 5.94\left(\mathrm{~s}, 1 \mathrm{H}\right.$, pyrazole), $3.11\left(\mathrm{t}, J=7.0 \mathrm{~Hz}, 2 \mathrm{H}, \mathrm{COCH}_{2}\right), 2.53\left(\mathrm{~s}, 3 \mathrm{H}, \mathrm{ArCH}_{3}\right), 2.24\left(\mathrm{~s}, 3 \mathrm{H}, \mathrm{ArCH}_{3}\right)$, 1.90-1.48 (m, 9H), 1.18-1.12(m, 2H) ; ${ }^{13} \mathrm{C} \mathrm{NMR}\left(125 \mathrm{MHz}, \mathrm{CDCl}_{3}\right) \delta 174.4,151.7,144.0,110.9,39.6,34.5$, 32.6, 30.6, 25.2, 14.6, 13.8; IR (thin film, NaCl) 2949, 2866, 1730, 1582, 1412, 1379, 1335, $963 \mathrm{~cm}^{-1}$ HRMS (ESI, H) $\mathrm{m} / \mathrm{z}$ calc'd for $\mathrm{C}_{13} \mathrm{H}_{21} \mathrm{~N}_{2} \mathrm{O} 221.1654(\mathrm{M}+\mathrm{H})^{+}$, found 221.1653

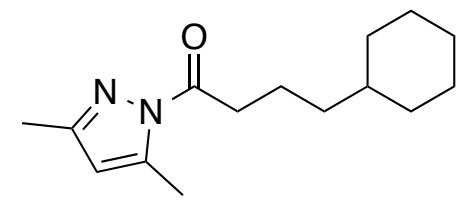

4-cyclohexyl-1-(3,5-dimethyl-1H-pyrazol-1-yl)butan-1-one (1f) (colorless oil); ${ }^{1} \mathrm{H}-\mathrm{NMR}\left(500 \mathrm{MHz}, \mathrm{CDCl}_{3}\right.$ ) 
(ppm) $\delta 5.94\left(\mathrm{~s}, 1 \mathrm{H}\right.$, pyrazole), $3.06\left(\mathrm{t}, J=7.5 \mathrm{~Hz}, 2 \mathrm{H}, \mathrm{COCH}_{2}\right), 2.53\left(\mathrm{~s}, 3 \mathrm{H}, \mathrm{ArCH}_{3}\right), 2.23\left(\mathrm{~s}, 3 \mathrm{H}, \operatorname{ArCH}_{3}\right)$, 1.77-1.62 (m, 7H), 1.30-1.10 (m, 6H), 0.93-0.86 (m, 2H) ; $\left.{ }^{13} \mathrm{C} \mathrm{NMR} \mathrm{(125} \mathrm{MHz,} \mathrm{CDCl}_{3}\right) \delta 174.3,151.7$, 144.0, 110.9, 37.4, 36.9, 35.4, 33.3, 26.7, 26.4, 21.7, 14.6, 13.8; IR (thin film, $\mathrm{NaCl}$ ) 2924, 2851, 1732, $1582,1447,1412,1379,1325,1240,963 \mathrm{~cm}^{-1}$; HRMS (ESI, H) $\mathrm{m} / z$ calc'd for $\mathrm{C}_{15} \mathrm{H}_{25} \mathrm{~N}_{2} \mathrm{O}(\mathrm{M}+\mathrm{H})^{+} 249.1961$, found 249.1966 .

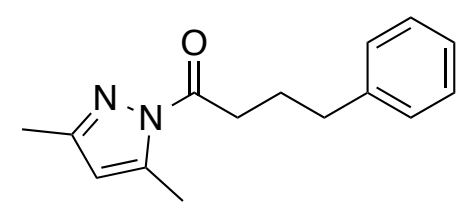

1-(3,5-dimethyl-1H-pyrazol-1-yl)-4-phenylbutan-1-one (1g) (white solid); ${ }^{1} \mathrm{H}-\mathrm{NMR}$ (500 $\mathrm{MHz}, \mathrm{CDCl}_{3}$ ) (ppm) $\delta$ 7.29-7.25 (m, 2H, Ph), 7.23-7.16 (m, 3H, Ph), 5.94 (s, 1H, pyrazole), 3.14 (t, J = $7.5 \mathrm{~Hz}, 2 \mathrm{H}$, $\left.\mathrm{COCH}_{2}\right), 2.73\left(\mathrm{t}, J=7.5 \mathrm{~Hz}, 2 \mathrm{H}, \mathrm{PhCH}_{2}\right), 2.53\left(\mathrm{~s}, 3 \mathrm{H}, \mathrm{ArCH}_{3}\right), 2.23\left(\mathrm{~s}, 3 \mathrm{H}, \mathrm{ArCH}_{3}\right), 2.08(\mathrm{t}, J=7.5 \mathrm{~Hz}, 2 \mathrm{H}$, $\left.\mathrm{CH}_{2}\right) ;{ }^{13} \mathrm{C} \mathrm{NMR}\left(125 \mathrm{MHz}, \mathrm{CDCl}_{3}\right) \delta 173.8,151.8,144.0,141.7,128.5,128.4,125.9,111.0,35.2,34.7,25.9$, 14.6, 13.8; IR (KBr) 3026, 2961, 2862, 1728, 1580, 1478, 1441, 1379, 1344, 1242, 993, 963, $920 \mathrm{~cm}^{-1}$; HRMS (ESI, Na) $m / z$ calc'd for $\mathrm{C}_{15} \mathrm{H}_{19} \mathrm{~N}_{2} \mathrm{O}(\mathrm{M}+\mathrm{H})^{+} 243.1492$, found 243.1499 .

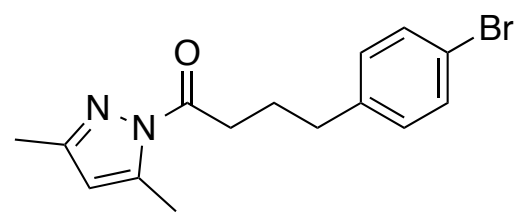

4-(4-bromophenyl)-1-(3,5-dimethyl-1H-pyrazol-1-yl)butan-1-one (1h) (white solid); ${ }^{1} \mathrm{H}-\mathrm{NMR}$ (500MHz, $\left.\mathrm{CDCl}_{3}\right)(\mathrm{ppm}) \delta 7.39$ (d, $\left.J=8.5 \mathrm{~Hz}, 2 \mathrm{H}, \mathrm{Ar}\right), 7.09$ (d, $\left.J=8.5 \mathrm{~Hz}, 2 \mathrm{H}, \mathrm{Ar}\right), 5.94$ (s, 1H, pyrazole), 3.12 (t, $J=7.0$ $\left.\mathrm{Hz}, 2 \mathrm{H}, \mathrm{COCH}_{2}\right), 2.68\left(\mathrm{t}, J=8.0 \mathrm{~Hz}, 2 \mathrm{H}, \mathrm{CH}_{2} \mathrm{Ar}\right), 2.52\left(\mathrm{~s}, 3 \mathrm{H}, \mathrm{ArCH}_{3}\right), 2.23\left(\mathrm{~s}, 3 \mathrm{H}, \mathrm{ArCH}_{3}\right), 2.05$ (q, J = $7.5 \mathrm{~Hz}$, $\left.2 \mathrm{H}, \mathrm{CH}_{2}\right) ;{ }^{13} \mathrm{C}$ NMR $\left(125 \mathrm{MHz}, \mathrm{CDCl}_{3}\right) \delta 173.2,151.4,143.5,140.1,130.9,129.8,119.2,110.6,34.1,34.0$, 25.3, 14.1, 13.3; IR (NaCl) 2928, 2862, 1728, 1584, 1431, 1384, 1312, 1072, 1011, $961 \mathrm{~cm}^{-1}$; HRMS (ESI, H) $m / z$ calc'd for $\mathrm{C}_{15} \mathrm{H}_{18} \mathrm{BrN}_{2} \mathrm{O}(\mathrm{M}+\mathrm{H})^{+} 321.0597$, found 321.0606 . 


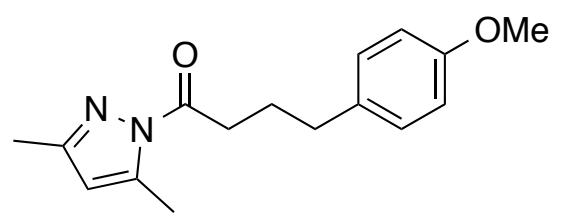

1-(3,5-dimethyl-1H-pyrzol-1-yl)-4-(4-methoxyphenyl)butan-1-one (1i) (colorless oil); ${ }^{1} \mathrm{H}-\mathrm{NMR}(500 \mathrm{MHz}$, $\left.\mathrm{CDCl}_{3}\right)(\mathrm{ppm}) \delta 7.13(\mathrm{~d}, J=8.5 \mathrm{~Hz}, 2 \mathrm{H}, \mathrm{Ar}), 6.82(\mathrm{~d}, J=8.5 \mathrm{~Hz}, 2 \mathrm{H}, \mathrm{Ar}), 5.94(\mathrm{~s}, 1 \mathrm{H}$, pyrazole), $3.78(\mathrm{~s}, 3 \mathrm{H}$, $\mathrm{OCH}_{3}$ ), $3.12\left(\mathrm{t}, J=7.5 \mathrm{~Hz} 2 \mathrm{H}, \mathrm{COCH}_{2}\right), 2.67\left(\mathrm{t}, J=7.5 \mathrm{~Hz}, 2 \mathrm{H}, \mathrm{ArCH}_{2}\right), 2.53\left(\mathrm{~s}, 3 \mathrm{H}, \mathrm{ArCH}_{3}\right), 2.23(\mathrm{~s}, 3 \mathrm{H}$, $\mathrm{ArCH}_{3}$ ), 2.04 (q, $\left.J=7.5 \mathrm{~Hz}, 2 \mathrm{H}, \mathrm{CH}_{2}\right) ;{ }^{13} \mathrm{CNMR}\left(125 \mathrm{MHz}, \mathrm{CDCl}_{3}\right) \delta 173.9,157.9,151.7,144.0,133.8,129.4$, $113.8,111.0,55.3,34.6,34.3,26.2,14.6,13.8$; IR (thin film, $\mathrm{NaCl}$ ) 2928, 1722, 1613, 1584, 1512, 1443, $1373,1319,1244,1175,1030,957,804 \mathrm{~cm}^{-1}$; HRMS (ESI, H) $\mathrm{m} / z$ calc'd for $\mathrm{C}_{16} \mathrm{H}_{21} \mathrm{~N}_{2} \mathrm{O}_{2}(\mathrm{M}+\mathrm{H})^{+} 273.1598$, found 273.1603 .

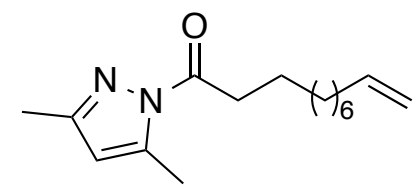

1-(3,5-dimethyl-1H-pyrazol-1-yl)undec-10-en-1-one (1j) (colorless oil); ${ }^{1} \mathrm{H}-\mathrm{NMR}\left(500 \mathrm{MHz}, \mathrm{CDCl}_{3}\right)(\mathrm{ppm})$

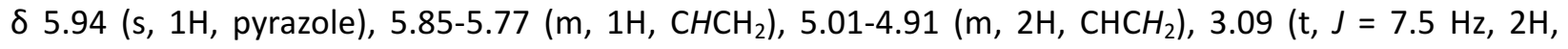
$\mathrm{COCH}_{2}$ ), $2.54\left(\mathrm{~s}, 3 \mathrm{H}, \mathrm{ArCH}_{3}\right), 2.24\left(\mathrm{~s}, 3 \mathrm{H}, \mathrm{ArCH}_{3}\right), 2.06-2.01\left(\mathrm{~m}, 2 \mathrm{H}, \mathrm{CH}_{2} \mathrm{CH}_{2} \mathrm{CH}\right), 1.73$ (quin, $J=7.5 \mathrm{~Hz}, 2 \mathrm{H}$, $\left.\mathrm{COCH}_{2} \mathrm{CH}_{2}\right), 1.42-1.28\left(\mathrm{~m}, 10 \mathrm{H}\right.$, alkyl chain); $\left.{ }^{13} \mathrm{C} \mathrm{NMR} \mathrm{(125} \mathrm{MHz,} \mathrm{CDCl}_{3}\right) \delta 174.2,151.7,144.0,139.2$, $114.1,110.9,35.2,33.8,29.3,29.12,29.09,29.0,28.9,24.3,14.6,13.8$; IR (thin film, NaCl) 2928, 2855, $1730,1641,1582,1412,1383,1344,963,910 \mathrm{~cm}^{-1}$; HRMS (ESI, H) $\mathrm{m} / \mathrm{z}$ calc'd for $\mathrm{C}_{16} \mathrm{H}_{27} \mathrm{~N}_{2} \mathrm{O}(\mathrm{M}+\mathrm{H})^{+}$ 263.2118, found 263.2111 .

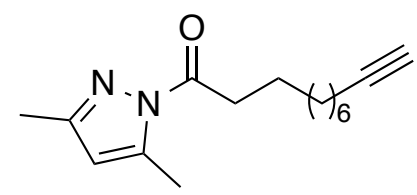

1-(3,5-dimethyl-1H-pyrazol-1-yl)undec-10-yn-1-one (1k) (colorless oil); ${ }^{1} \mathrm{H}-\mathrm{NMR}\left(500 \mathrm{MHz}, \mathrm{CDCl}_{3}\right)(\mathrm{ppm})$ $\delta 5.94\left(\mathrm{~s}, 1 \mathrm{H}\right.$, pyrazole), 3.09 (t, J = $7.5 \mathrm{~Hz}, 2 \mathrm{H}, \mathrm{COCH}_{2}$ ), $2.54\left(\mathrm{~s}, 3 \mathrm{H}, \mathrm{ArCH}_{3}\right), 2.24\left(\mathrm{~s}, 3 \mathrm{H}, \mathrm{ArCH}_{3}\right), 2.18$ (dt, J $=7.5,3.0 \mathrm{~Hz}, 2 \mathrm{H}, \mathrm{CH}_{2} \mathrm{CCH}$ ), $1.93\left(\mathrm{t}, J=3.0 \mathrm{~Hz}, 1 \mathrm{H}, \mathrm{CH}_{2} \mathrm{CCH}\right.$ ), 1.73 (q, $J=7.5 \mathrm{~Hz}, 2 \mathrm{H}, \mathrm{CH}_{2}$ ), 1.52 (q, $J=7.5$ $\left.\mathrm{Hz}, 2 \mathrm{H}, \mathrm{CH}_{2}\right), 1.43-1.31$ (m, 8H, alkyl chain); $\left.{ }^{13} \mathrm{C} \mathrm{NMR} \mathrm{(125} \mathrm{MHz,} \mathrm{CDCl}_{3}\right) \delta 174.2,151.7,144.0,110.9,84.8$, 
68.1, 35.1, 29.2, 29.1, 28.9, 28.7, 28.5, 24.3, 18.4, 14.6, 13.8; IR (KBr) 3248, 2923, 2853, 1726, 1468, $1385,1333,1312,964,839 \mathrm{~cm}^{-1}$; HRMS $(E S I, H) \mathrm{m} / z$ calc'd for $\mathrm{C}_{16} \mathrm{H}_{25} \mathrm{~N}_{2} \mathrm{O}(\mathrm{M}+\mathrm{H})^{+} 261.1961$, found 261.1967.

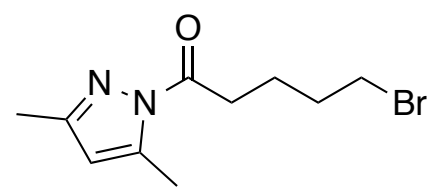

5-bromo-1-(3,5-dimethyl-1H-pyrazol-1-yl)pentan-1-one (1l) (white solid); ${ }^{1} \mathrm{H}-\mathrm{NMR}$ (500MHz, $\mathrm{CDCl}_{3}$ ) (ppm) $\delta 5.95$ (s, 1H, pyrazole), 3.56 (t, $J=6.5 \mathrm{~Hz}, 2 \mathrm{H}, \mathrm{CH}_{2} \mathrm{CH}_{2} \mathrm{Br}$ ), 3.46 (t, $J=6.5 \mathrm{~Hz}, 2 \mathrm{H}, \mathrm{CH}_{2} \mathrm{CH}_{2} \mathrm{Br}$ ), 3.14 (t, $\left.J=7.5,2 \mathrm{H}, \mathrm{COCH}_{2}\right), 2.54\left(\mathrm{~s}, 3 \mathrm{H}, \mathrm{ArCH}_{3}\right), 2.23\left(\mathrm{~s}, 3 \mathrm{H}, \mathrm{ArCH}_{3}\right), 2.01-1.87(\mathrm{~m}, 4 \mathrm{H}) ;{ }^{13} \mathrm{C} \mathrm{NMR}\left(125 \mathrm{MHz}, \mathrm{CDCl}_{3}\right)$ $\delta x x x ; \operatorname{IR~}(\mathrm{KBr}) 3111,2949,2926,1724,1584,1454,1387,1363,1329,1319,964,841 \mathrm{~cm}^{-1}$; HRMS (ESI, H) $\mathrm{m} / \mathrm{z}$ calc'd for $\mathrm{C}_{10} \mathrm{H}_{16} \mathrm{BrN}_{2} \mathrm{O}(\mathrm{M}+\mathrm{H})^{+} 259.0441$ found 259.0435

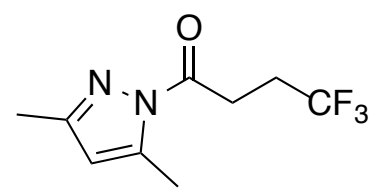

1-(3,5-dimethyl-1H-pyrazol-1-yl)-4,4,4-trifluorobutan-1-one (1m) (colorless oil); ${ }^{1} \mathrm{H}-\mathrm{NMR}$ (500MHz, $\left.\mathrm{CDCl}_{3}\right)(\mathrm{ppm}) \delta 5.98\left(\mathrm{~s}, 1 \mathrm{H}\right.$, pyrazole), $3.40\left(\mathrm{~m}, 2 \mathrm{H}, \mathrm{C}(\mathrm{O}) \mathrm{CH}_{2} \mathrm{CH}_{2}\right), 2.60-2.52\left(\mathrm{~m}, 5 \mathrm{H}, \mathrm{ArCH}_{3}, \mathrm{CH}_{2} \mathrm{CF}_{3}\right), 2.24$ (s, $\left.3 \mathrm{H}, \mathrm{ArCH}_{3}\right) ;{ }^{13} \mathrm{CNMR}\left(125 \mathrm{MHz}, \mathrm{CDCl}_{3}\right) \delta 171.0,152.6,144.2,126.8(J=275 \mathrm{~Hz}), 111.4$, , $28.7(J=29.9$ $\mathrm{Hz}), 28.5(\mathrm{~J}=2.88 \mathrm{~Hz}), 14.4,13.8$; IR (KBr) 2934, 1730, 1587, 1414, 1379, 1356, 1310, 1263, 1142, 982, $963 \mathrm{~cm}^{-1}$; HRMS (ESI, H) $\mathrm{m} / z$ calc'd for $\mathrm{C}_{9} \mathrm{H}_{12} \mathrm{~F}_{3} \mathrm{~N}_{2} \mathrm{O}(\mathrm{M}+\mathrm{H})^{+} 221.0896$, found 221.0909 .

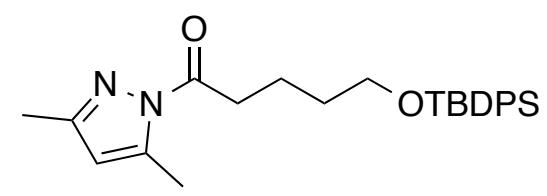

5-((tert-butyldiphenylsilyl)oxy)-1-(3,5-dimethyl-1H-pyrazol-1-yl)pentan-1-one (1n) (white solid); ${ }^{1} \mathrm{H}$ NMR (500 MHz, $\mathrm{CDCl}_{3}$ ) (ppm) ס 7.68-7.66 (m, 4H, Ph), 7.42-7.35 (m, 6H, Ph), 5.94 (s, 1H, pyrazole), 3.71 $\left(\mathrm{t}, J=6.5 \mathrm{~Hz}, 2 \mathrm{H}, \mathrm{OCH}_{2}\right), 3.10\left(\mathrm{t}, J=7.5 \mathrm{~Hz}, 2 \mathrm{H}, \mathrm{COCH}_{2}\right), 2.53\left(\mathrm{~s}, 3 \mathrm{H}, \mathrm{ArCH}_{3}\right), 2.23\left(\mathrm{~s}, 3 \mathrm{H}, \mathrm{ArCH}_{3}\right), 1.87-1.81$ $\left(\mathrm{m}, 2 \mathrm{H}, \mathrm{CH}_{2}\right), 1.69-1.63\left(\mathrm{~m}, 2 \mathrm{H}, \mathrm{CH}_{2}\right), 1.04\left(\mathrm{~s}, 9 \mathrm{H}, \mathrm{C}\left(\mathrm{CH}_{3}\right)_{3}\right) ;{ }^{13} \mathrm{C} \mathrm{NMR}\left(125 \mathrm{MHz}, \mathrm{CDCl}_{3}\right) \delta$ 174.0, 151.7, 
143.9, 135.6, 134.0, 129.5, 127.6, 110.9, 63.5, 35.0, 32.0, 26.7, 20.8, 19.2, 14.6, 13.8; IR (KBr) 2957, 2934, 2913, 2859, 1736, 1580, 1427, 1387, 1371, 1358, 1327, 1111, 1084, 988, $963 \mathrm{~cm}^{-1}$; HRMS (ESI, H) $m / z$ calc'd for $\mathrm{C}_{26} \mathrm{H}_{35} \mathrm{~N}_{2} \mathrm{O}_{2} \mathrm{Si}(\mathrm{M}+\mathrm{H})^{+} 435.2462$, found 435.2469 .

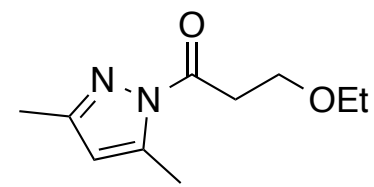

1-(3,5-dimethyl-1H-pyrazol-1-yl)-3-ethoxypropan-1-one (10) (colorless oil); ${ }^{1} \mathrm{H}-\mathrm{NMR}\left(500 \mathrm{MHz}, \mathrm{CDCl}_{3}\right.$ ) (ppm) $\delta 5.95\left(\mathrm{~s}, 1 \mathrm{H}\right.$, pyrazole), $3.84\left(\mathrm{t}, J=6.5 \mathrm{~Hz}, 2 \mathrm{H}, \mathrm{COCH}_{2} \mathrm{CH}_{2} \mathrm{O}\right.$ ), 3.54 (q, $\left.J=7.0 \mathrm{~Hz}, 2 \mathrm{H}, \mathrm{OCH}_{2} \mathrm{CH}_{3}\right), 3.38$ $\left(\mathrm{t}, J=6.5 \mathrm{~Hz}, 2 \mathrm{H}, \mathrm{COCH}_{2}\right), 2.54\left(\mathrm{~s}, 3 \mathrm{H}, \mathrm{ArCH}_{3}\right), 2.23\left(\mathrm{~s}, 3 \mathrm{H}, \mathrm{ArCH}_{3}\right), 1.20\left(\mathrm{t}, J=7.0 \mathrm{~Hz}, 2 \mathrm{H}, \mathrm{OCH}_{2} \mathrm{CH}_{3}\right) ;{ }^{13} \mathrm{C}$ NMR $\left(125 \mathrm{MHz}, \mathrm{CDCl}_{3}\right) \delta 172.0,152.0,144.0,111.1,66.4,65.4,35.9,15.1,14.5,13.8 ; \mathrm{IR}(\mathrm{KBr}) 2972$, $2886,1724,1478,1418,1387,1350,1240,1117,963 \mathrm{~cm}^{-1}$; HRMS $(E S I, H) \mathrm{m} / z$ calc'd for $\mathrm{C}_{10} \mathrm{H}_{17} \mathrm{~N}_{2} \mathrm{O}_{2}(\mathrm{M}+$ $\mathrm{Na})^{+} 219.1109$, found 219.1104 .

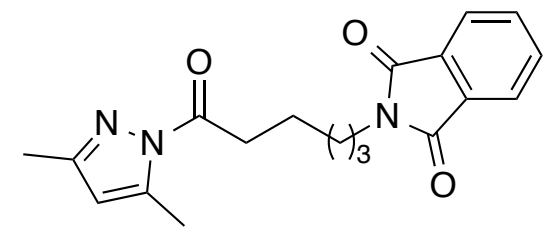

2-(6-(3,5-dimethyl-1H-pyrazol-1-yl)-6-oxohexyl)isoindoline-1,3-dione (1p) (white solid); ${ }^{1} \mathrm{H}-\mathrm{NMR}$ $\left(500 \mathrm{MHz}, \mathrm{CDCl}_{3}\right)(\mathrm{ppm}) \delta 7.84(\mathrm{dd}, J=5.5,3.0 \mathrm{~Hz}, 2 \mathrm{H}, \mathrm{Ar}), 7.71$ (dd, $\left.J=5.5,3.0 \mathrm{~Hz}, 2 \mathrm{H}, \mathrm{Ar}\right), 5.93(\mathrm{~s}, 1 \mathrm{H}$, pyrazole), $3.70\left(\mathrm{t}, J=7.0 \mathrm{~Hz}, 2 \mathrm{H}, \mathrm{CH}_{2} \mathrm{~N}\right), 3.10\left(\mathrm{t}, J=7.0 \mathrm{~Hz}, 2 \mathrm{H}, \mathrm{COCH}_{2}\right), 2.52\left(\mathrm{~s}, 3 \mathrm{H}, \mathrm{ArCH}_{3}\right), 2.22(\mathrm{~s}, 3 \mathrm{H}$, $\left.\mathrm{ArCH}_{3}\right), 1.81-1.71\left(\mathrm{~m}, 4 \mathrm{H}\right.$, alkyl chain), 1.49-1.43 (m, 2H, alkyl chain); $\left.{ }^{13} \mathrm{C} \mathrm{NMR} \mathrm{(125} \mathrm{MHz,} \mathrm{CDCl}_{3}\right) \delta 173.8$, $168.4,151.8,144.0,133.9,132.2,123.2,111.0,37.9,35.0,28.5,26.4,23.9,14.6,13.8 ; \operatorname{IR}(\mathrm{KBr}) 2945$, 2928, 1769, 1724, 1703, 1579, 1437, 1397, 1377, 1327, 1316, 1254, 1040, 961, $916 \mathrm{~cm}^{-1}$; HRMS (ESI, H) $m / z$ calc'd for $\mathrm{C}_{19} \mathrm{H}_{22} \mathrm{~N}_{3} \mathrm{O}_{3}(\mathrm{M}+\mathrm{H})^{+} 340.1656$, found 340.1665 . 


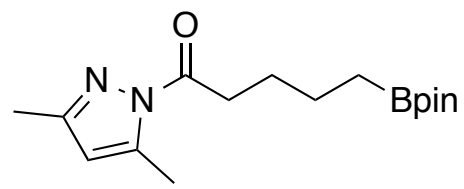

1-(3,5-dimethyl-1H-pyrazol-1-yl)-5-(4,4,5,5-tetramethyl-1,3,2-dioxaborolan-2-yl)pentan-1-one

(1q)

(colorless oil); ${ }^{1} \mathrm{H}-\mathrm{NMR}\left(500 \mathrm{MHz}, \mathrm{CDCl}_{3}\right)(\mathrm{ppm}) \delta 5.93(\mathrm{~s}, 1 \mathrm{H}$, pyrazole), $3.09(\mathrm{t}, J=7.5 \mathrm{~Hz}, 2 \mathrm{H}$, $\mathrm{C}(\mathrm{O}) \mathrm{CH}_{2} \mathrm{CH}_{2}$ ), $2.53\left(\mathrm{~s}, 3 \mathrm{H}, \mathrm{ArCH}_{3}\right.$ ), $2.22\left(\mathrm{~s}, 3 \mathrm{H}, \mathrm{ArCH}_{3}\right.$ ), 1.74 (quin, $J=7.5 \mathrm{~Hz}, 2 \mathrm{H}, \mathrm{C}(\mathrm{O}) \mathrm{CH}_{2} \mathrm{CH}_{2}$ ), 1.53 (quin, $J$ $=7.5 \mathrm{~Hz} 2 \mathrm{H}$ overlapped with water, $\left.\mathrm{CH}_{2} \mathrm{CH}_{2} \mathrm{~B}\right), 1.24\left(\mathrm{~s}, 12 \mathrm{H}, \mathrm{OC}\left\{\left(\mathrm{CH}_{3}\right)_{2}\right\} \mathrm{C}\left\{\left(\mathrm{CH}_{3}\right)_{2}\right\} \mathrm{O}\right), 0.83(\mathrm{t}, J=8.0,2 \mathrm{H}$, $\left.\mathrm{CH}_{2} \mathrm{CH}_{2} \mathrm{~B}\right) ;{ }^{13} \mathrm{C}$ NMR $\left(125 \mathrm{MHz}, \mathrm{CDCl}_{3}\right) \delta 174.2,151.6,143.9,110.9,82.9,35.1,26.9,24.8,23.7,14.6,13.8$, 11.0; IR (thin film, NaCl) 2980, 2932, 2251, 1728, 1379, 1321, 1146, 964, 910, $847 \mathrm{~cm}^{-1}$; HRMS (ESI, H) $m / z$ calc'd for $\mathrm{C}_{16} \mathrm{H}_{28} \mathrm{BN}_{2} \mathrm{O}_{3}(\mathrm{M}+\mathrm{H})^{+} 307.2187$, found.307.2193

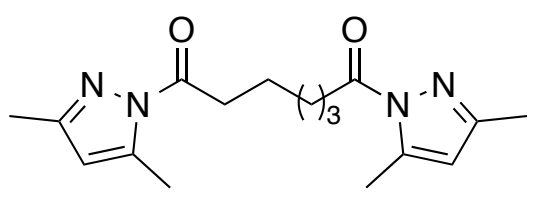

1,7-bis(3,5-dimethyl-1H-pyrazol-1-yl)heptane-1,7-dione (1r) (white solid); ${ }^{1} \mathrm{H}-\mathrm{NMR}$ (500MHz, $\mathrm{CDCl}_{3}$ ) (ppm) $\delta 5.94$ (s, 2H, pyrazole), $3.12\left(\mathrm{t}, J=7.5 \mathrm{~Hz}, 4 \mathrm{H}, \mathrm{COCH}_{2}\right), 2.53\left(\mathrm{~s}, 6 \mathrm{H}, \mathrm{ArCH}_{3}\right), 2.23\left(\mathrm{~s}, 6 \mathrm{H}, \mathrm{ArCH}_{3}\right), 1.80$ (q, $\left.J=7.5 \mathrm{~Hz}, 4 \mathrm{H}, \mathrm{COCH}_{2} \mathrm{CH}_{2}\right), 1.56-1.49(\mathrm{~m}, 2 \mathrm{H}) ;{ }^{13} \mathrm{C} \mathrm{NMR}\left(125 \mathrm{MHz}, \mathrm{CDCl}_{3}\right) \delta 174.0,151.8,144.0,111.0$, 35.0, 28.6, 24.1, 14.6, 13.8; IR (KBr) 3115, 2923, 1874 1732, 1586, 1412, 1387, 1337, 1312, 1263, 959, $849 \mathrm{~cm}^{-1} ; \mathrm{HRMS}(\mathrm{ESI}, \mathrm{H}) \mathrm{m} / z$ calc'd for $\mathrm{C}_{17} \mathrm{H}_{25} \mathrm{~N}_{4} \mathrm{O}_{2}(\mathrm{M}+\mathrm{H})^{+}$317.1972, found 317.1975.

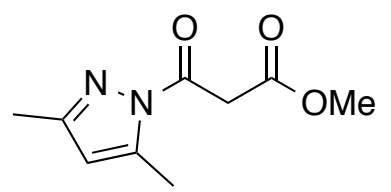

methyl 3-(3,5-dimethyl-1H-pyrazol-1-yl)-3-oxopropanoate (1s) (colorless oil); ${ }^{1} \mathrm{H}-\mathrm{NMR}\left(500 \mathrm{MHz}, \mathrm{CDCl}_{3}\right.$ ) (ppm) $\delta 5.97\left(\mathrm{~s}, 1 \mathrm{H}\right.$, pyrazole), $4.11\left(\mathrm{~s}, 2 \mathrm{H}, \mathrm{COCH}_{2}\right), 3.76\left(\mathrm{~s}, 3 \mathrm{H}, \mathrm{OCH}_{3}\right), 2.55\left(\mathrm{~s}, 3 \mathrm{H}, \mathrm{ArCH}_{3}\right), 2.21(\mathrm{~s}, 3 \mathrm{H}$, $\left.\mathrm{ArCH}_{3}\right) ;{ }^{13} \mathrm{C} \mathrm{NMR}\left(125 \mathrm{MHz}, \mathrm{CDCl}_{3}\right) \delta 167.6,166.6,152.7,144.3,111.8,52.5,42.8,14.3,13.8$; IR (thin film, NaCl) 3341, 2955, 2870, 2577, 1748, 1586, 1439, 1358, 1333, 1279, 1153, 1022, $964 \mathrm{~cm}^{-1}$; HRMS (ESI, Na) $m / z$ calc'd for $\mathrm{C}_{9} \mathrm{H}_{12} \mathrm{~N}_{2} \mathrm{NaO}_{3}(\mathrm{M}+\mathrm{Na})^{+} 219.0740$, found 219.0745 . 


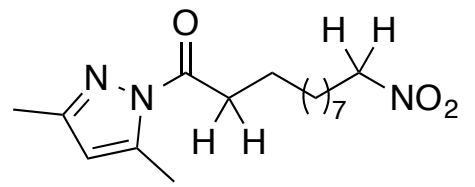

1-(3,5-dimethyl-1H-pyrazol-1-yl)-11-nitroundecan-1-one (1t) (white solid); ${ }^{1} \mathrm{H}-\mathrm{NMR}$ (500MHz, $\mathrm{CDCl}_{3}$ ) (ppm) $\delta 5.94\left(\mathrm{~s}, 1 \mathrm{H}\right.$, pyrazole), $4.37\left(\mathrm{t}, J=7.0 \mathrm{~Hz}, 2 \mathrm{H}, \mathrm{CH}_{2} \mathrm{NO}_{2}\right), 3.09\left(\mathrm{t}, J=7.5 \mathrm{~Hz}, 2 \mathrm{H}, \mathrm{COCH}_{2}\right), 2.54(\mathrm{~s}, 3 \mathrm{H}$, $\mathrm{ArCH}_{3}$ ), $2.24\left(\mathrm{~s}, 3 \mathrm{H}, \mathrm{ArCH}_{3}\right), 2.00\left(\mathrm{q}, J=7.0 \mathrm{~Hz}, 2 \mathrm{H}, \mathrm{CH}_{2} \mathrm{CH}_{2} \mathrm{NO}_{2}\right.$ ), 1.72 (q, J= 7.5 Hz, 2H, $\mathrm{COCH}_{2} \mathrm{CH}_{2}$ ), $1.41-$ $1.30\left(\mathrm{~m}, 12 \mathrm{H}\right.$, alkyl chain); ${ }^{13} \mathrm{C}$ NMR $\left(125 \mathrm{MHz}, \mathrm{CDCl}_{3}\right) \delta$ 174.2, 151.7, 144.0, 110.9, 75.7, 35.1, 29.29, 29.25, 29.2, 29.1, 28.8, 27.4, 26.2, 24.3, 14.6, 13.8; IR (KBr) 2922, 2853, 1723, 1584, 1551, 1474, 1410, 1391, 1321, 1250, $961 \mathrm{~cm}^{-1}$; HRMS (ESI, H) m/z calc'd for $\mathrm{C}_{16} \mathrm{H}_{28} \mathrm{~N}_{3} \mathrm{O}_{3}(\mathrm{M}+\mathrm{H})^{+} 310.2125$, found 310.2130.

\section{Procedure for the synthesis of $1 \mathrm{t}\left(d^{2}\right)^{3}$}

A $20 \mathrm{~mL}$ shlenk flask equipped with a magnetic stirring bar and 3-way glass stopcock was charged with 1t $(62.0 \mathrm{mg}, 0.2 \mathrm{mmol})$, THF $(2.0 \mathrm{ml})$ and $\mathrm{D}_{2} \mathrm{O}(2.0 \mathrm{ml})$. To the solution was added triethylamine $(5.6 \mu \mathrm{l}$, $0.04 \mathrm{mmol})$. The resulting mixture was stirred at the same temperature for $24 \mathrm{~h}$ at room temperature. The crude reaction mixture was directly purified by silica gel column chromatography ( $n$-hexane: EtOAc $=$ $6: 1)$ to give the desired product) to give desired product $1 \mathbf{t}\left(d^{2}\right)$.

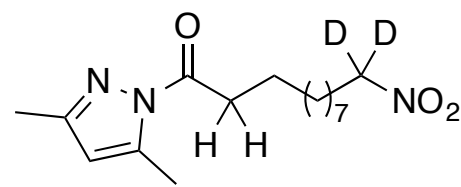

1-(3,5-dimethyl-1H-pyrazol-1-yl)-11-nitroundecan-1-one-11,11-d2 $\quad\left(1 \mathrm{t}\left(\boldsymbol{d}^{2}\right)\right) \quad$ (white solid); ${ }^{1} \mathrm{H}-\mathrm{NMR}$ (500MHz, CDCl $\left.{ }_{3}\right)(\mathrm{ppm}) \delta 5.94\left(\mathrm{~s}, 1 \mathrm{H}\right.$, pyrazole), 3.09 (t, $\left.J=7.5 \mathrm{~Hz}, 2 \mathrm{H}, \mathrm{COCH}_{2}\right), 2.54\left(\mathrm{~s}, 3 \mathrm{H}, \operatorname{ArCH}_{3}\right), 2.24$ $\left(\mathrm{s}, 3 \mathrm{H}, \mathrm{ArCH}_{3}\right), 2.00\left(\mathrm{t}, J=7.0 \mathrm{~Hz}, 2 \mathrm{H}, \mathrm{CH}_{2} \mathrm{CD}_{2} \mathrm{NO}_{2}\right), 1.73\left(\mathrm{q}, J=7.5 \mathrm{~Hz}, 2 \mathrm{H}, \mathrm{COCH}_{2} \mathrm{CH}_{2}\right), 1.41-1.30(\mathrm{~m}, 12 \mathrm{H}$, alkyl chain) ; ${ }^{13} \mathrm{C}$ NMR $\left(125 \mathrm{MHz}, \mathrm{CDCl}_{3}\right) \delta^{3} \mathrm{C} \mathrm{NMR}\left(125 \mathrm{MHz}, \mathrm{CDCl}_{3}\right) \delta 174.2,151.7,144.0,110.9$, $75.1(\mathrm{~J}=$ $22.0 \mathrm{~Hz}$ ), 35.1, 29.3, 29.25, 29.19, 29.06, 28.82, 27.2, 26.2, 24.3, 14.6, 13.8; IR (KBr) 2922, 2853, 1723, 1539, 1474, 1391, 1329, $961 \mathrm{~cm}^{-1}$; HRMS $(E S I, H) \mathrm{m} / z$ calc'd for $\mathrm{C}_{16} \mathrm{H}_{26} \mathrm{D}_{2} \mathrm{~N}_{3} \mathrm{O}_{3}(\mathrm{M}+\mathrm{H})^{+} 312.2251$, found 312.2252 . 


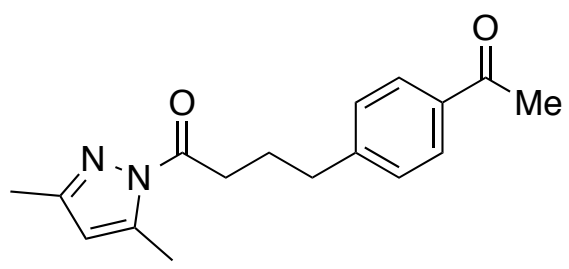

4-(4-acetylphenyl)-1-(3,5-dimethyl-1H-pyrazol-1-yl)butan-1-one (1u) (white solid) ; ${ }^{1} \mathrm{H}-\mathrm{NMR}$ (500 MHz, $\left.\mathrm{CDCl}_{3}\right)(\mathrm{ppm}) \delta 7.88(\mathrm{~d}, J=8.0 \mathrm{~Hz}, 2 \mathrm{H}, \mathrm{Ar}), 7.31(\mathrm{~d}, J=8.0 \mathrm{~Hz}, 2 \mathrm{H}, \mathrm{Ar}), 5.95(\mathrm{~s}, 1 \mathrm{H}$, pyrazole), $3.14(\mathrm{t}, J=7.5$ $\left.\mathrm{Hz}, 2 \mathrm{H}, \mathrm{COCH}_{2}\right), 2.79\left(\mathrm{t}, J=7.5 \mathrm{~Hz}, 2 \mathrm{H}, \mathrm{ArCH}_{2}\right), 2.58\left(\mathrm{~s}, 3 \mathrm{H}, \mathrm{COCH}_{3}\right), 2.53\left(\mathrm{~s}, 3 \mathrm{H}, \mathrm{ArCH}_{3}\right), 2.23(\mathrm{~s}, 3 \mathrm{H}$, $\left.\mathrm{ArCH}_{3}\right) 2.10\left(\mathrm{~m}, 2 \mathrm{H}, \mathrm{CH}_{2}\right) ;{ }^{13} \mathrm{C} \mathrm{NMR}\left(125 \mathrm{MHz}, \mathrm{CDCl}_{3}\right) \delta 197.8,173.6,151.9,147.5,144.0,135.2,128.7$, 128.6, 111.1, 35.1, 34.5, 26.6, 25.5, 14.6, 13.8; IR (KBr) 3750, 3648, 2926, 1728, 1682, 1604, 1584, 1391, 1351, 1329, 1267, $964 \mathrm{~cm}^{-1}$; HRMS (ESI, Na) $\mathrm{m} / z$ calc'd for $\mathrm{C}_{17} \mathrm{H}_{21} \mathrm{~N}_{2} \mathrm{O}_{2}(\mathrm{M}+\mathrm{H})^{+}$285.1598, found 285.1595 .

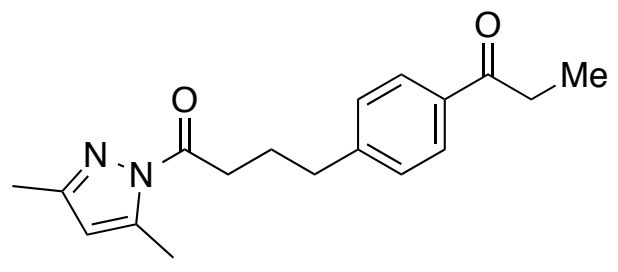

1-(3,5-dimethyl-1H-pyrazol-1-yl)-4-(4-propionylphenyl)butan-1-one (1v) (pale yellow solid): ${ }^{1} \mathrm{H}-\mathrm{NMR}$ $\left(500 \mathrm{MHz}, \mathrm{CDCl}_{3}\right)(\mathrm{ppm}) \delta 7.89$ (d, J = $8.5 \mathrm{~Hz}, 2 \mathrm{H}, \mathrm{Ar}$ ), 7.31 (d, J = 8.5 Hz, 2H, Ar), 5.95 (s, 1H, pyrazole), $3.14\left(\mathrm{t}, J=7.5 \mathrm{~Hz}, 2 \mathrm{H}, \mathrm{COCH}_{2}\right), 3.00-2.96(\mathrm{~m}, 2 \mathrm{H}), 2.79\left(\mathrm{t}, J=8.0 \mathrm{~Hz}, 2 \mathrm{H}, \mathrm{CH}_{2}\right), 2.53\left(\mathrm{~s}, 3 \mathrm{H}, \mathrm{ArCH}_{3}\right), 2.23(\mathrm{~s}$, $\left.3 \mathrm{H}, \mathrm{ArCH}_{3}\right), 2.13-2.06\left(\mathrm{~m}, 2 \mathrm{H}, \mathrm{CH}_{2}\right), 1.22\left(\mathrm{t}, J=7.5 \mathrm{~Hz}, 3 \mathrm{H}, \mathrm{CH}_{3}\right) ;{ }^{13} \mathrm{C} \mathrm{NMR}\left(125 \mathrm{MHz}, \mathrm{CDCl}_{3}\right) \delta$ 200.6, 173.6, $151.9,147.2$, 144.0, 135.0, 128.7, 128.2, 111.1, 35.1, 34.5, 31.7, 25.6, 14.6, 13.8, 8.33; IR (KBr) 2970, 2934, 1728, 1682, 1607, 1410, 1375, 1323, 1252, 1224, 1211, 1181, $957 \mathrm{~cm}^{-1}$; HRMS (ESI, H) m/z calc'd for $\mathrm{C}_{18} \mathrm{H}_{23} \mathrm{~N}_{2} \mathrm{O}_{2}(\mathrm{M}+\mathrm{H})^{+} 299.1754$, found 299.1760 .

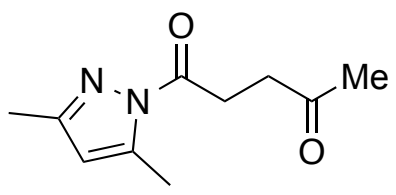

1-(3,5-dimethyl-1H-pyrazol-1-yl)pentane-1,4-dione (1w) (white solid): ${ }^{1} \mathrm{H}-\mathrm{NMR}\left(500 \mathrm{MHz}, \mathrm{CDCl}_{3}\right)(\mathrm{ppm})$ $\delta 5.95\left(\mathrm{~s}, 1 \mathrm{H}\right.$, pyrazole), $3.39\left(\mathrm{t}, J=6.0 \mathrm{~Hz}, 2 \mathrm{H}, \mathrm{COCH}_{2}\right), 2.85\left(\mathrm{t}, J=6.0 \mathrm{~Hz}, 2 \mathrm{H}, \mathrm{COCH}_{2}\right), 2.51\left(\mathrm{~s}, 3 \mathrm{H}, \mathrm{ArCH}_{3}\right)$, $2.24\left(\mathrm{~s}, 3 \mathrm{H}, \mathrm{CH}_{3}\right), 2.23\left(\mathrm{~s}, 3 \mathrm{H}, \mathrm{CH}_{3}\right) ;{ }^{13} \mathrm{C}$ NMR $\left(125 \mathrm{MHz}, \mathrm{CDCl}_{3}\right) \delta 206.7,172.9,152.1,144.0,111.1,37.3$, 
29.9, 29.5, 14.4, 13.8; IR (KBr) 3119, 2924, 1724, 1587, 1487, 1437, 1375, 1317, 1260, 1188, 1161, 1145, 999, 959, $831 \mathrm{~cm}^{-1}$; HRMS (ESI, H) $\mathrm{m} / z$ calc'd for $\mathrm{C}_{10} \mathrm{H}_{15} \mathrm{~N}_{2} \mathrm{O}_{2}(\mathrm{M}+\mathrm{H})^{+}$195.1128, found 195.1134 .

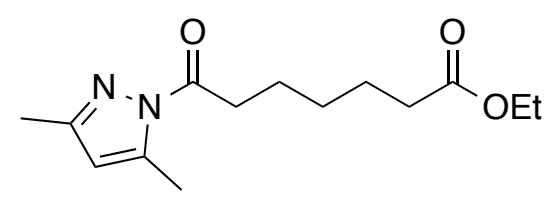

ethyl 7-(3,5-dimethyl-1H-pyrazol-1-yl)-7-oxoheptanoate (1x) (colorless oil) ; ${ }^{1} \mathrm{H}-\mathrm{NMR}\left(500 \mathrm{MHz}, \mathrm{CDCl}_{3}\right.$ ) (ppm) $\delta 5.94\left(\mathrm{~s}, 1 \mathrm{H}\right.$, pyrazole), $4.12\left(\mathrm{q}, J=7.5 \mathrm{~Hz}, 2 \mathrm{H}, \mathrm{OCH}_{2}\right), 3.10\left(\mathrm{t}, J=8.0 \mathrm{~Hz}, 2 \mathrm{H}, \mathrm{COCH}_{2}\right), 2.53(\mathrm{~s}, 3 \mathrm{H}$, $\mathrm{ArCH}_{3}$ ), $2.32\left(\mathrm{t}, J=7.5 \mathrm{~Hz}, 2 \mathrm{H}, \mathrm{COCH}_{2}\right.$ ), $2.23\left(\mathrm{~s}, 3 \mathrm{H}, \mathrm{ArCH}_{3}\right.$ ), 1.75 (quin, $J=7.5 \mathrm{~Hz}, 2 \mathrm{H}$ ), 1.69 (quin, $J=7.5$ $\mathrm{Hz}, 2 \mathrm{H}), 1.46-1.42(\mathrm{~m}, 2 \mathrm{H}), 1.25\left(\mathrm{t}, J=7.5 \mathrm{~Hz}, 3 \mathrm{H}, \mathrm{CH}_{3}\right) ;{ }^{13} \mathrm{C} \mathrm{NMR}\left(125 \mathrm{MHz}, \mathrm{CDCl}_{3}\right) \delta 173.9,173.7,151.8$, 144.0, 111.0, 60.2, 35.0, 34.2, 28.6, 24.7, 23.9, 14.6, 14.2, 13.8; IR (thin film, NaCl) 2932, 2667, 1730, 1712, 1582, 1445, 1413, 1385, 1344, 1312, 1176, 1141, 1096, 1032, $961 \mathrm{~cm}^{-1}$; HRMS (ESI, H) m/z calc'd for $\mathrm{C}_{14} \mathrm{H}_{23} \mathrm{~N}_{2} \mathrm{O}_{3}(\mathrm{M}+\mathrm{H})^{+} 267.1703$, found 267.1709.

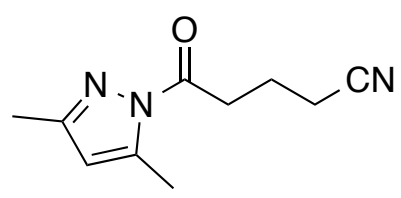

5-(3,5-dimethyl-1H-pyrazol-1-yl)-5-oxopentanenitrile (1y) (white solid) ; ${ }^{1} \mathrm{H}-\mathrm{NMR}$ (500 $\mathrm{MHz}, \mathrm{CDCl}_{3}$ ) (ppm) $\delta 5.97\left(\mathrm{~s}, 1 \mathrm{H}\right.$, pyrazole), $3.28\left(\mathrm{t}, J=7.0 \mathrm{~Hz}, 2 \mathrm{H}, \mathrm{COCH}_{2}\right), 2.54-2.51\left(\mathrm{~m}, 5 \mathrm{H}, \mathrm{CH}_{2}\right.$ and $\left.\mathrm{ArCH}_{3}\right), 2.23(\mathrm{~s}$, $3 \mathrm{H}, \mathrm{ArCH}_{3}$ ), 2.10 (quin, $J=7.0 \mathrm{~Hz}, 2 \mathrm{H}, \mathrm{CH}_{2}$ ); ${ }^{13} \mathrm{C} \mathrm{NMR}\left(125 \mathrm{MHz} \mathrm{CDCl}_{3}\right) \delta 172.2,152.3,144.0,119.2$, 111.3, 33.6, 20.3, 16.5, 14.4, 13.7; IR (KBr) 3725, 3628, 2972, 2945, 2932, 2241, 1726, 1582, 1481, 1437, 1391, 1348, 1252, $964 \mathrm{~cm}^{-1}$; HRMS (ESI, Na) $\mathrm{m} / z$ calc'd for $\mathrm{C}_{10} \mathrm{H}_{14} \mathrm{~N}_{3} \mathrm{O}(\mathrm{M}+\mathrm{H})^{+} 192.1131$, found 192.1133<smiles>Cc1cc(C)n(C(=O)CCCS(=O)(=O)c2ccccc2)n1</smiles>

1-(3,5-dimethyl-1H-pyrazol-1-yl)-4-(phenylsulfonyl)butan-1-one (1z) (white solid); ${ }^{1} \mathrm{H}-\mathrm{NMR}$ (500MHz, $\left.\mathrm{CDCl}_{3}\right)(\mathrm{ppm}) \delta 7.94(\mathrm{~d}, J=8.5 \mathrm{~Hz}, 2 \mathrm{H}, \mathrm{Ar}), 7.66(\mathrm{tt}, J=7.5,1.5 \mathrm{~Hz}, 1 \mathrm{H}, \mathrm{Ar}), 7.58(\mathrm{t}, J=7.5 \mathrm{~Hz}, 2 \mathrm{H}, \mathrm{Ar}), 5.94$ (s, $1 \mathrm{H}$, pyrazole), 3.27-3.22 (m, $\left.4 \mathrm{H}, \mathrm{COCH}_{2} \mathrm{CH}_{2} \mathrm{SO}_{2}\right), 2.50\left(\mathrm{~s}, 3 \mathrm{H}, \mathrm{ArCH}_{3}\right), 2.20\left(\mathrm{~s}, 3 \mathrm{H}, \mathrm{ArCH}_{3}\right), 2.17-2.10(\mathrm{~m}$, 
$\left.2 \mathrm{H}, \mathrm{COCH}_{2} \mathrm{CH}_{2} \mathrm{CH}_{2}\right) ;{ }^{13} \mathrm{C}$ NMR $\left(125 \mathrm{MHz}, \mathrm{CDCl}_{3}\right) \delta 172.3,152.3,144.0,139.0,133.7,129.3,128.2,111.3$, 55.3, , 33.4, 17.9, 14.4, 13.8; IR (KBr) 2962, 2928, 1726, 1582, 1452, 1414, 1385, 1346, 1314, 1150, 961 $\mathrm{cm}^{-1}$; HRMS (ESI, H) $\mathrm{m} / z$ calc'd for $\mathrm{C}_{15} \mathrm{H}_{19} \mathrm{~N}_{2} \mathrm{O}_{3} \mathrm{~S}(\mathrm{M}+\mathrm{H})^{+} 307.1111$, found 307.1112 .

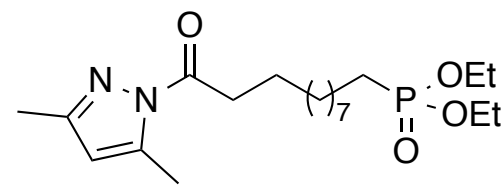

diethyl (11-(3,5-dimethyl-1H-pyrazol-1-yl)-11-oxoundecyl)phosphonate (1 $\alpha)$ (colorless oil); ${ }^{1} \mathrm{H}-\mathrm{NMR}$ $\left(500 \mathrm{MHz}, \mathrm{CDCl}_{3}\right)(\mathrm{ppm}) \delta 5.94\left(\mathrm{~s}, 1 \mathrm{H}\right.$, pyrazole), 4.15-4.03 (m, 4H, $\left.\mathrm{P}(\mathrm{O})\left(\mathrm{OCH}_{2} \mathrm{CH}_{3}\right)_{2}\right), 3.09$ (t, $J=7.5 \mathrm{~Hz}$, $\left.2 \mathrm{H}, \mathrm{COCH}_{2}\right), 2.54\left(\mathrm{~s}, 3 \mathrm{H}, \mathrm{ArCH}_{3}\right), 2.24\left(\mathrm{~s}, 3 \mathrm{H}, \mathrm{ArCH}_{3}\right), 1.75-1.54(\mathrm{~m}, 6 \mathrm{H}$, overlapped with water), 1.40-1.28 $(\mathrm{m}, 18 \mathrm{H}) ;{ }^{13} \mathrm{C}$ NMR $\left(125 \mathrm{MHz}, \mathrm{CDCl}_{3}\right) \delta 174.2,151.7,144.0,110.9,61.4(J=6.49 \mathrm{~Hz}), 35.2,30.6(J=68.3$ $\mathrm{Hz}), 16.5(\mathrm{~J}=24.0 \mathrm{~Hz})$, ; $^{31} \mathrm{P} \mathrm{NMR}\left(200 \mathrm{MHz}, \mathrm{CDCl}_{3}\right) \delta 32.7 \mathrm{ppm}$; IR $(\mathrm{KBr}) 2962,2928,1726,1582,1452$, $1414,1385,1346,1314,1150,961 \mathrm{~cm}^{-1}$; $\operatorname{HRMS}(\mathrm{ESI}, \mathrm{H}) \mathrm{m} / z$ calc'd for $\mathrm{C}_{20} \mathrm{H}_{38} \mathrm{~N}_{2} \mathrm{O}_{4} \mathrm{P}(\mathrm{M}+\mathrm{H})^{+} 401.2564$, found 401.2570 .

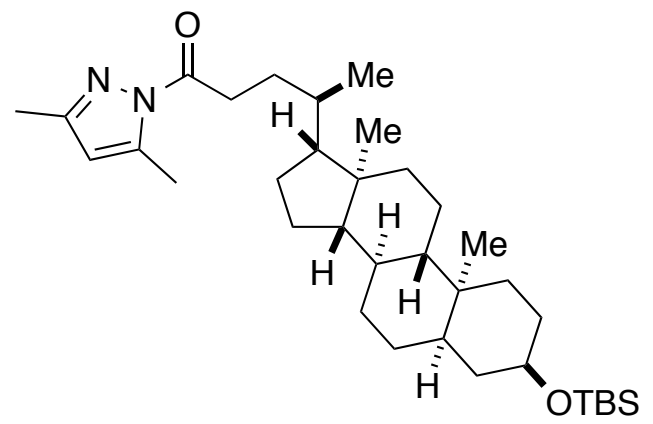

(R)-4-((3R,5R,8R,9S,10S,13R,14S,17R)-3-((tert-butyldimethylsilyl)oxy)-10,13-dimethylhexadecahydro-

1H-cyclopenta[a]phenanthren-17-yl)-1-(3,5-dimethyl-1H-pyrazol-1-yl)pentan-1-one (1ß) (white solid); ${ }^{1} \mathrm{H}-\mathrm{NMR}\left(500 \mathrm{MHz}, \mathrm{CDCl}_{3}\right)$ (ppm) $\delta 5.80\left(\mathrm{~s}, 1 \mathrm{H}\right.$, pyrazole), 3.61-3.55 $(\mathrm{m}, 1 \mathrm{H}), 3.01-3.02\left(\mathrm{~m}, 1 \mathrm{H}, \mathrm{COCH}_{2}\right)$, 3.00-2.93 (m, 1H, $\left.\mathrm{COCH}_{2}\right), 1.91-1.67(\mathrm{~m}, 6 \mathrm{H}), 1.55-0.97(\mathrm{~m}, 22 \mathrm{H}), 0.91(\mathrm{~d}, J=6.5 \mathrm{~Hz}, 3 \mathrm{H}), 0.84(\mathrm{~s}, 3 \mathrm{H})$, $0.84(\mathrm{~s}, 4 \mathrm{H}), 0.83\left(\mathrm{~s}, 9 \mathrm{H}, \mathrm{C}\left(\mathrm{CH}_{3}\right)_{3}\right), 0.59(\mathrm{~s}, 3 \mathrm{H}) ;{ }^{13} \mathrm{CNMR}\left(125 \mathrm{MHz}, \mathrm{CDCl}_{3}\right)$ 8174.7, 151.6, 143.9, 110.9, 72.8, 56.4, 56.0, 42.8, 42.3, 40.2, 40.1, 37.0, 35.9, 35.6, 35.4, 34.6, 32.2, 31.1, 30.4, 28.2 , 27.3, 26.4, 26.0, 24.3, 23.4, 20.8, 18.6, 18.3, 14.6, 13.8, 12.0, -4.57; IR (KBr) 2928, 2883, 2858, 1732, 1582, 1462, 1449, 1377, 1341, 1252, 1096, 1080, $961 \mathrm{~cm}^{-1}$; HRMS (ESI, H) $\mathrm{m} / z$ calc'd for $\mathrm{C}_{35} \mathrm{H}_{61} \mathrm{~N}_{2} \mathrm{O}_{2} \mathrm{Si}(\mathrm{M}+\mathrm{H})^{+} 569.4496$, 
found 569.4502 .

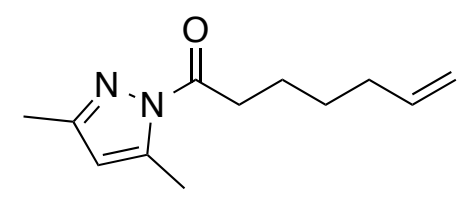

1-(3,5-dimethyl-1 H-pyrazol-1-yl)hept-6-en-1-one (1 $\gamma$ ) (colorless oil); ${ }^{1} \mathrm{H}-\mathrm{NMR}\left(500 \mathrm{MHz}, \mathrm{CDCl}_{3}\right)(\mathrm{ppm})$ $\delta 5.94\left(\mathrm{~s}, 1 \mathrm{H}\right.$, pyrazole), 5.86-5.78 (m, $\left.1 \mathrm{H}, \mathrm{CHCH}_{2}\right), 5.04-4.94\left(\mathrm{~m}, 2 \mathrm{H}, \mathrm{CHCH}_{2}\right), 3.11(\mathrm{t}, J=7.5 \mathrm{~Hz}, 2 \mathrm{H}$, $\left.\mathrm{COCH}_{2}\right), 2.54\left(\mathrm{~s}, 3 \mathrm{H}, \mathrm{ArCH}_{3}\right), 2.24\left(\mathrm{~s}, 3 \mathrm{H}, \mathrm{ArCH}_{3}\right), 2.11(\mathrm{~m}, 2 \mathrm{H}), 1.79-1.72(\mathrm{~m}, 2 \mathrm{H}), 1.54-1.47(\mathrm{~m}, 2 \mathrm{H}) ;{ }^{13} \mathrm{C}$ NMR $\left(125 \mathrm{MHz}, \mathrm{CDCl}_{3}\right) \delta 174.0,151.7,144.0,138.5,114.6,110.9,35.0,33.5,28.3,23.8,14.6,13.8 ; \mathrm{IR}$ (thin film, NaCl) 3077, 2930, 2861, 1730, 1642, 1582, 1142, 1081, 1344, 1246, 963, $912 \mathrm{~cm}^{-1}$; HRMS (ESI, $\mathrm{Na}) \mathrm{m} / \mathrm{z}$ calc'd for $\mathrm{C}_{12} \mathrm{H}_{19} \mathrm{~N}_{2} \mathrm{O}(\mathrm{M}+\mathrm{H})^{+} 207.1492$ found 207.1495 .

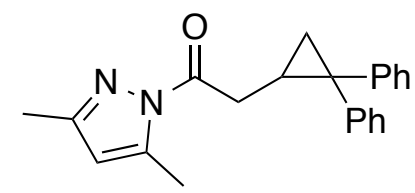

1-(3,5-dimethyl-1H-pyrazol-1-yl)-2-(2,2-diphenylcyclopropyl)ethan-1-one (18) ${ }^{4} \quad$ (white solid); ${ }^{1} \mathrm{H}-\mathrm{NMR}$ $\left(500 \mathrm{MHz}, \mathrm{CDCl}_{3}\right)(\mathrm{ppm}) \delta 7.41(\mathrm{~m}, 2 \mathrm{H}, \mathrm{Ar}), 7.34(\mathrm{~m}, 2 \mathrm{H}, \mathrm{Ar}), 7.26-7.20(\mathrm{~m}, 4 \mathrm{H}, \mathrm{Ar}), 7.15-7.11(\mathrm{~m}, 2 \mathrm{H}, \mathrm{Ar})$, $5.89\left(\mathrm{~s}, 1 \mathrm{H}\right.$, pyrazole), 3.02-2.85 (m, 2H, $\left.\mathrm{COCH}_{2}\right), 2.56\left(\mathrm{~s}, 3 \mathrm{H}, \mathrm{ArCH}_{3}\right), 2.16-2.10\left(\mathrm{~m}, 4 \mathrm{H}, \mathrm{ArCH}_{3}\right.$, $\left.\mathrm{CH}_{2} \mathrm{CH}\left(\mathrm{CH}_{2}\right) \mathrm{C}\right), 1.41-1.39(\mathrm{t}, J=5.5 \mathrm{~Hz}, 1 \mathrm{H}), 1.34-1.31(\mathrm{~m}, 1 \mathrm{H}) ;{ }^{13} \mathrm{C} \mathrm{NMR}\left(125 \mathrm{MHz}, \mathrm{CDCl}_{3}\right) \delta 173.6,151.6$, 146.9, 143.8, 141.3, 130.2, 128.4, 128.3, 128.2, 126.5, 125.9, 110.9, 36.7, 35.4, 20.9, 18.7, 14.6, 13.7.; IR $(\mathrm{KBr}) \mathrm{cm}^{-1} 3017,1723,1584,1495,1412,1377,1354,1303,1242,963$; HRMS (ESI, H) $\mathrm{m} / z$ calc'd for $\mathrm{C}_{22} \mathrm{H}_{23} \mathrm{~N}_{2} \mathrm{O}(\mathrm{M}+\mathrm{H})^{+} 331.1810$ found 331.1805 .

\section{General Procedure and Characterization of the Products}

General procedure for catalytic $\alpha$-amination of acylpyrazoles: A $20 \mathrm{~mL}$ shlenk flask equipped with a magnetic stirring bar and 3-way glass stopcock was charged with 4A crushed molecular sieves (MA 4A $100.0 \mathrm{mg}$ ) and flamed-dried under vacumm. After cooling down to room temperature, $\mathrm{Cu}(\mathrm{OTf})_{2}(7.2 \mathrm{mg}$, $0.02 \mathrm{mmol}$ ) was added to the flask under inert atmosphere in a dry box. To the flask was added dry 
$\mathrm{CH}_{2} \mathrm{Cl}_{2}(0.2 \mathrm{~mL})$ via syringe with stainless-steel needle followed by the addition of acylpyrazole 1 (0.4 mmol). Then iminoiodinane $2(0.2 \mathrm{mmol})$ was added to the mixture as a solid at $0{ }^{\circ} \mathrm{C}$. The resulting greenish suspension was stirred at the same temperature for $48 \mathrm{~h}$ under Ar and diluted with EtOAc. The diluted solution was filtered through silica short column and washed with EtOAc (ca. $30 \mathrm{ml}$ ). After evaporation of the organic solvent under reduced pressure, the crude mixture was purified by silica gel column chromatography to give the desired product 3.

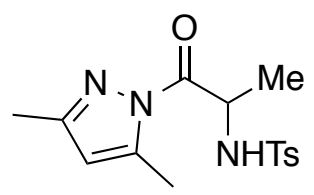

$N$-(1-(3,5-dimethyl-1H-pyrazol-1-yl)-1-oxopropan-2-yl)-4-methylbenzenesulfonamide (3a) (white solid, 20\% EtOAc/n-hexane, 76\% yield); ${ }^{1} \mathrm{H}-\mathrm{NMR}\left(500 \mathrm{MHz}, \mathrm{CDCl}_{3}\right)(\mathrm{ppm}) \delta 7.63(\mathrm{~d}, J=8.5 \mathrm{~Hz}, 2 \mathrm{H}, \mathrm{Ar}), 7.13(\mathrm{~d}, J$ $=8.5 \mathrm{~Hz}, 2 \mathrm{H}, \mathrm{Ar}), 5.89(\mathrm{~s}, 1 \mathrm{H}$, pyrazole), $5.67(\mathrm{~d}, J=10.5 \mathrm{~Hz}, 1 \mathrm{H}, \mathrm{NH}), 5.15-5.08(\mathrm{~m}, 1 \mathrm{H}, \mathrm{CHN}), 2.34(\mathrm{~s}, 3 \mathrm{H}$, $\mathrm{ArCH}_{3}$ ), $2.22\left(\mathrm{~s}, 6 \mathrm{H}, \mathrm{ArCH}_{3}\right), 1.48\left(\mathrm{~d}, \mathrm{~J}=7.0 \mathrm{~Hz}, 3 \mathrm{H}, \mathrm{C}(\mathrm{O}) \mathrm{CH}_{2} \mathrm{CH}_{3}\right) ;{ }^{13} \mathrm{C} \mathrm{NMR}\left(125 \mathrm{MHz}, \mathrm{CDCl}_{3}\right) \delta 172.2$, 153.1, 144.4, 143.4, 136.6, 129.3, 127.3, 111.4, 52.4, 21.4, 20.3, 13.83, 13.81; IR (KBr) 3271, 1730, 1587, $1385,1354,1329,1165,1147,1092,947 \mathrm{~cm}^{-1}$; HRMS (ESI, Na) $\mathrm{m} / z$ calc'd for $\mathrm{C}_{15} \mathrm{H}_{20} \mathrm{~N}_{3} \mathrm{O}_{3} \mathrm{~S}+(\mathrm{M}+\mathrm{H})^{+}$ 322.1220, found 322.1220 .

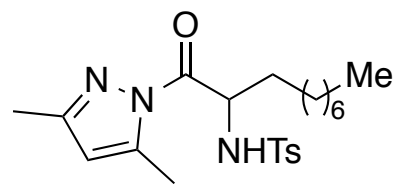

N-(1-(3,5-dimethyl-1H-pyrazol-1-yl)-1-oxodecan-2-yl)-4-methylbenzenesulfonamide (3b) (white solid, 20\% EtOAc/n-hexane, 76\% yield) ; ${ }^{1} \mathrm{H}-\mathrm{NMR}\left(500 \mathrm{MHz}, \mathrm{CDCl}_{3}\right)(\mathrm{ppm}) \delta 7.61$ (d, J = 8.5 Hz, 2H, Ar), 7.10 (d, J $=8.5 \mathrm{~Hz}, 2 \mathrm{H}, \mathrm{Ar}), 5.87(\mathrm{~s}, 1 \mathrm{H}$, pyrazole), $5.63(\mathrm{~d}, J=11.0 \mathrm{~Hz}, 1 \mathrm{H}, \mathrm{NH}), 5.00-4.95(\mathrm{~m}, 1 \mathrm{H}, \mathrm{CHN}), 2.33(\mathrm{~s}, 3 \mathrm{H}$, $\left.\mathrm{ArCH}_{3}\right), 2.22\left(\mathrm{~s}, 3 \mathrm{H}, \mathrm{ArCH}_{3}\right), 2.19\left(\mathrm{~s}, 3 \mathrm{H}, \mathrm{ArCH}_{3}\right), 1.86-1.78(\mathrm{~m}, 1 \mathrm{H}), 1.65-1.59(\mathrm{~m}, 1 \mathrm{H}$, overlapped with $\left.\mathrm{H}_{2} \mathrm{O}\right), 1.46-1.39(\mathrm{~m}, 2 \mathrm{H}), 1.30-1.23(\mathrm{~m}, 11 \mathrm{H}), 0.87\left(\mathrm{t}, J=7.0 \mathrm{~Hz}, 3 \mathrm{H}, \mathrm{CH}_{2} \mathrm{CH}_{3}\right) ;{ }^{13} \mathrm{C} \mathrm{NMR}\left(125 \mathrm{MHz}, \mathrm{CDCl}_{3}\right) \delta$ 171.9, 152.9, 144.3, 143.3, 136.5, 129.2, 127.3, 111.3, 56.5, 33.5, 31.8, 29.24, 29.17, 28.8, 25.2, 22.6, 21.4, 14.1, 13.8; IR (KBr) 3262, 2922, 2851, 1732, 1377, 1356, 1331 1163, $961 \mathrm{~cm}^{-1}$; HRMS (ESI, Na) m/z calc'd for $\mathrm{C}_{22} \mathrm{H}_{34} \mathrm{~N}_{3} \mathrm{O}_{3} \mathrm{~S}(\mathrm{M}+\mathrm{H})^{+} 420.2315$, found 420.2322 
<smiles>Cc1cc(C)n(C(=O)C(N)CC(C)C)n1</smiles>

$N$-(1-(3,5-dimethyl-1H-pyrazol-1-yl)-4-methyl-1-oxopentan-2-yl)-4-methylbenzenesulfonamide

(white solid, 20\% EtOAc/n-hexane, 63\% yield); ${ }^{1} \mathrm{H}-\mathrm{NMR}\left(500 \mathrm{MHz}, \mathrm{CDCl}_{3}\right)(\mathrm{ppm}) \delta 7.60$ (d, $J=8.5 \mathrm{~Hz}, 2 \mathrm{H}$, Ar- $H$ ), 7.08 (d, $J=8.5 \mathrm{~Hz}, 2 \mathrm{H}, \operatorname{Ar}-H), 5.86(\mathrm{~s}, 1 \mathrm{H}$, pyrazole), 5.60 (d, $J=11.5 \mathrm{~Hz}, 1 \mathrm{H}, \mathrm{C}(\mathrm{O}) \mathrm{CHNH}$ ), 5.06-5.01 $(\mathrm{m}, 1 \mathrm{H}, \mathrm{C}(\mathrm{O}) \mathrm{CHNH}), 2.32\left(\mathrm{~s}, 3 \mathrm{H}, \mathrm{ArCH}_{3}\right), 2.23\left(\mathrm{~s}, 3 \mathrm{H}, \mathrm{ArCH}_{3}\right), 2.15\left(\mathrm{~s}, 3 \mathrm{H}, \mathrm{ArCH}_{3}\right), 1.92(\mathrm{~m}, 1 \mathrm{H}$, $\left.\left.\mathrm{CH}_{2} \mathrm{CH}\left(\mathrm{CH}_{2}\right) \mathrm{CH}_{2}\right), 1.56\left(\mathrm{t}, J=7.5 \mathrm{~Hz}, 2 \mathrm{H}, \mathrm{CHCH}_{2} \mathrm{CH}\right), 0.98\left(\mathrm{~d}, J=6.5 \mathrm{~Hz}, 3 \mathrm{H}, \mathrm{CH}\left(\mathrm{CH}_{3}\right) \mathrm{CH}_{3}\right)\right), 0.93(\mathrm{~d}, J=6.5$ $\left.\left.\mathrm{Hz}, 3 \mathrm{H}, \mathrm{CH}\left(\mathrm{CH}_{3}\right) \mathrm{CH}_{3}\right)\right) ;{ }^{13} \mathrm{C}$ NMR (125 MHz, $\left.\mathrm{CDCl}_{3}\right) \delta 1742.1$, 152.8, 144.2, 143.2, 136.4, 129.2, 127.3, 111.2, 55.4, 42.4, 24.7, 23.1, 21.4, 21.0, 13.84, 13.75; IR (KBr) 3277, 2955, 2872, 1728, 1589, 1379, 1356, 1331, 1302, 1165, 1090, $963 \mathrm{~cm}^{-1}$; HRMS (ESI, H) m/z calc'd for $\mathrm{C}_{18} \mathrm{H}_{26} \mathrm{~N}_{3} \mathrm{O}_{3} \mathrm{~S}(\mathrm{M}+\mathrm{H})^{+} 364.1695$, found 364.1695 .

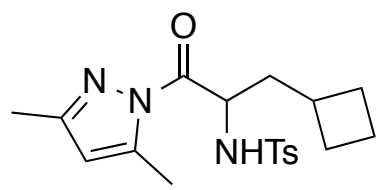

$N$-(3-cyclobutyl-1-(3,5-dimethyl-1H-pyrazol-1-yl)-1-oxopropan-2-yl)-4-methylbenzenesulfonamide (3d) (white solid, 20\% EtOAc/n-hexane, 63\% yield); ${ }^{1} \mathrm{H}-\mathrm{NMR}\left(500 \mathrm{MHz}, \mathrm{CDCl}_{3}\right)(\mathrm{ppm}) \delta 7.60(\mathrm{~d}, J=8.0 \mathrm{~Hz}, 2 \mathrm{H}$, Ar), $7.10(\mathrm{~d}, J=8.0 \mathrm{~Hz}, 2 \mathrm{H}, \operatorname{Ar}), 5.87(\mathrm{~s}, 1 \mathrm{H}$, pyrazole), $5.60(\mathrm{~d}, J=10.5 \mathrm{~Hz}, 1 \mathrm{H}, \mathrm{NH}), 4.93-4.88(\mathrm{~m}, 1 \mathrm{H}$, $\mathrm{COCH}), 2.56-2.46(\mathrm{~m}, 1 \mathrm{H}), 2.32\left(\mathrm{~s}, 3 \mathrm{H}, \mathrm{ArCH}_{3}\right), 2.23\left(\mathrm{~s}, 3 \mathrm{H}, \mathrm{ArCH}_{3}\right), 2.18\left(\mathrm{~s}, 3 \mathrm{H}, \mathrm{ArCH}_{3}\right), 2.08-1.53(\mathrm{~m}, 8 \mathrm{H}$ overlapped with water); ${ }^{13} \mathrm{C}$ NMR $\left(125 \mathrm{MHz} \mathrm{CDCl}_{3}\right) \delta 171.3,152.9,144.2,143.2,136.5,129.1,127.3$, $111.2,55.5,40.6,32.4,28.4,27.8,21.4,18.5,13.9,13.8$; IR (KBr) 3275, 2965, 1730, 1587, 1379, 1354, $1331,1306,1165,1090,963,816 \mathrm{~cm}^{-1}$; HRMS (ESI, H) $\mathrm{m} / z$ calc'd for $\mathrm{C}_{19} \mathrm{H}_{26} \mathrm{~N}_{3} \mathrm{O}_{3} \mathrm{~S}(\mathrm{M}+\mathrm{H})^{+} 376.1689$, found 376.1693 .

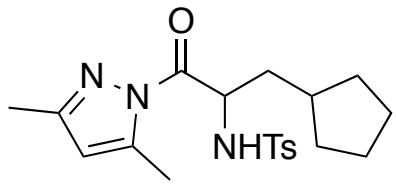

$N$-(3-cyclopentyl-1-(3,5-dimethyl-1H-pyrazol-1-yl)-1-oxopropan-2-yl)-4-methylbenzenesulfonamide (3e) (white solid, 20\% EtOAc/n-hexane, 60\% yield); ${ }^{1} \mathrm{H}-\mathrm{NMR}\left(500 \mathrm{MHz}, \mathrm{CDCl}_{3}\right)(\mathrm{ppm}) \delta 7.61$ (d, J $=8.5 \mathrm{~Hz}$, 
$2 \mathrm{H}, \operatorname{Ar}-\mathrm{H}), 7.10(\mathrm{~d}, J=8.5 \mathrm{~Hz}, 2 \mathrm{H}, \mathrm{Ar}), 5.87$ (s, $1 \mathrm{H}$, pyrazole), 5.59 (d, J = 10.5, 1H, NH), 4.98-4.93 (m, 1H), $2.33\left(\mathrm{~s}, 3 \mathrm{H}, \mathrm{ArCH}_{3}\right), 2.22\left(\mathrm{~s}, 3 \mathrm{H}, \mathrm{ArCH}_{3}\right), 2.19\left(\mathrm{~s}, 3 \mathrm{H}, \mathrm{ArCH}_{3}\right), 1.89-1.82(\mathrm{~m}, 1 \mathrm{H}), 1.70-1.58(\mathrm{~m}, 6 \mathrm{H}), 1.38-$ $1.07(\mathrm{~m}, 6 \mathrm{H}), 0.88-0.76(\mathrm{~m}, 2 \mathrm{H}) ;{ }^{13} \mathrm{C}$ NMR $\left(125 \mathrm{MHz} \mathrm{CDCl}_{3}\right) \delta 172.0,152.8,144.2,143.2,136.5,129.2$, $127.3,111.2,56.5,39.8,36.5,32.9,31.6,25.2,25.0,21.4,13.85,13.79 ; \mathrm{IR}(\mathrm{KBr}) 3289,2920,2851,1732$, $1586,1377,1350,1325,1159,1092,961,814 \mathrm{~cm}^{-1}$ : HRMS (ESI, H) $\mathrm{m} / \mathrm{z}$ calc'd for $\mathrm{C}_{22} \mathrm{H}_{32} \mathrm{~N}_{3} \mathrm{O}_{3} \mathrm{~S}(\mathrm{M}+\mathrm{H})^{+}$ 390.1846 , found 390.1851

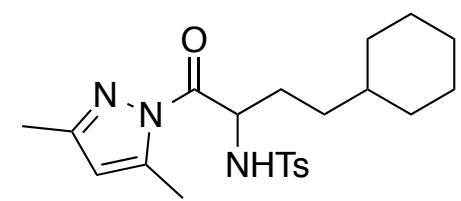

$N$-(4-cyclohexyl-1-(3,5-dimethyl-1H-pyrazol-1-yl)-1-oxobutan-2-yl)-4-methylbenzenesulfonamide (white solid, 20\% EtOAc/n-hexane, 72\% yield); ${ }^{1} \mathrm{H}-\mathrm{NMR}\left(500 \mathrm{MHz}, \mathrm{CDCl}_{3}\right)(\mathrm{ppm}) \delta 7.61(\mathrm{~d}, J=8.5 \mathrm{~Hz}, 2 \mathrm{H}$, Ar), $7.10(\mathrm{~d}, J=8.5 \mathrm{~Hz}, 2 \mathrm{H}, \mathrm{Ar}), 5.87$ (s, 1H, pyrazole), 5.59 (d, $J=10.5,1 \mathrm{H}, \mathrm{NH}), 4.98-4.93(\mathrm{~m}, 1 \mathrm{H}), 2.33$ (s, $\left.3 \mathrm{H}, \mathrm{ArCH}_{3}\right), 2.22\left(\mathrm{~s}, 3 \mathrm{H}, \mathrm{ArCH}_{3}\right), 2.19\left(\mathrm{~s}, 3 \mathrm{H}, \mathrm{ArCH}_{3}\right), 1.89-1.82(\mathrm{~m}, 1 \mathrm{H}), 1.70-1.58(\mathrm{~m}, 6 \mathrm{H}), 1.38-1.07(\mathrm{~m}$, $6 \mathrm{H}), 0.88-0.76(\mathrm{~m}, 2 \mathrm{H}) ;{ }^{13} \mathrm{C} \mathrm{NMR}\left(125 \mathrm{MHz} \mathrm{CDCl}_{3}\right) \delta 171.9,152.9,144.3,143.3,136.6,129.2,127.3$, 111.3, 56.6, 36.9, 33.3, 32.8, 32.7, 31.0, 26.6, 26.29, 26.25, 21.4, 13.8 (overlapped); IR (KBr) 3289, 2920, 2851, 1732, 1586, 1377, 1350, 1325, 1159, 1092, 961, $814 \mathrm{~cm}^{-1}$; HRMS (ESI, H) $\mathrm{m} / \mathrm{z}$ calc'd for $\mathrm{C}_{22} \mathrm{H}_{32} \mathrm{~N}_{3} \mathrm{O}_{3} \mathrm{~S}(\mathrm{M}+\mathrm{H})^{+} 418.2164$, found 418.2163

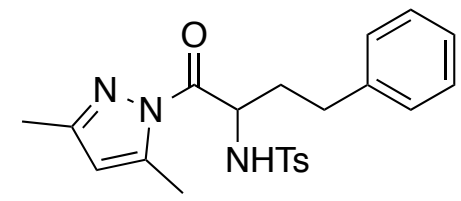

N-(1-(3,5-dimethyl-1H-pyrazol-1-yl)-1-oxo-4-phenylbutan-2-yl)-4-methylbenzenesulfonamide

(white solid, 11\%-20\% EtOAc/n-hexane, 78\% yield) ; ${ }^{1} \mathrm{H}-\mathrm{NMR}\left(500 \mathrm{MHz}, \mathrm{CDCl}_{3}\right)(\mathrm{ppm}) \delta 7.52(\mathrm{~d}, J=8.0$ $\mathrm{Hz}, 2 \mathrm{H}, \mathrm{Ar}$ ), 7.17 (d, J = 8.0 Hz, 2H, Ar), 7.19-7.02 (m, 5H, Ar), 5.79 (s, 1H, pyrazole), 5.68 (d, J = $10.5 \mathrm{~Hz}$, $1 \mathrm{H}, \mathrm{NH}), 4.98-4.92(\mathrm{~m}, 1 \mathrm{H}, \mathrm{CH}), 2.83-2.77\left(\mathrm{~m}, 1 \mathrm{H}, \mathrm{CH}_{2}\right), 2.69-2.62\left(\mathrm{~m}, 1 \mathrm{H}, \mathrm{CH}_{2}\right), 2.26\left(\mathrm{~s}, 3 \mathrm{H}, \operatorname{ArCH}_{3}\right), 2.19$ $\left(\mathrm{s}, 3 \mathrm{H}, \mathrm{ArCH}_{3}\right), 2.00-1.96\left(\mathrm{~m}, 4 \mathrm{H}, \mathrm{ArCH}_{3}, \mathrm{CH}_{2}\right), 1.93-1.85\left(\mathrm{~m}, 1 \mathrm{H}, \mathrm{CH}_{2}\right) ;{ }^{13} \mathrm{C} \mathrm{NMR}\left(125 \mathrm{MHz}, \mathrm{CDCl}_{3}\right) \delta 171.4$, 153.1, 144.3, 143.4, 140.6, 136.4, 129.3, 128.5, 128.3, 127.3, 126.0, 111.3, 56.5, 35.2, 31.6, 21.4, 13.80, 13.75; IR (KBr) 3268, 3028, 2926, 1728, 1588, 1454, 1381, 1360, 1333, 1306, 1163, 1092, $963 \mathrm{~cm}^{-1} ; \mathrm{H}$; HRMS (ESI, H) $m / z$ calc'd for $\mathrm{C}_{22} \mathrm{H}_{26} \mathrm{~N}_{3} \mathrm{O}_{3} \mathrm{~S}(\mathrm{M}+\mathrm{H})^{+} 412.1689$, found 412.1695. 
<smiles>Cc1cc(C)n(C(=O)C(CCc2ccc(Br)cc2)NS)n1</smiles>

N-(4-(4-bromophenyl)-1-(3,5-dimethyl-1H-pyrazol-1-yl)-1-oxobutan-2-yl)-4-

methylbenzenesulfonamide (3h) (white solid, $14 \% \mathrm{EtOAc} / n$-hexane, $72 \%$ yield); ${ }^{1} \mathrm{H}-\mathrm{NMR}$ (500MHz, $\left.\mathrm{CDCl}_{3}\right)(\mathrm{ppm}) \delta 7.60(\mathrm{~d}, J=8.0 \mathrm{~Hz}, 2 \mathrm{H}, \mathrm{Ar}), 7.37(\mathrm{~d}, J=8.0 \mathrm{~Hz}, 2 \mathrm{H}, \mathrm{Ar}), 7.10(\mathrm{~d}, J=8.0 \mathrm{~Hz}, 2 \mathrm{H}, \mathrm{Ar}), 7.04(\mathrm{~d}, J=$ $8.0 \mathrm{~Hz}, 2 \mathrm{H}, \mathrm{Ar}$ ), 5.86 (s, 1H, pyrazole), 5.71 (d, $J=10.5 \mathrm{~Hz}, 1 \mathrm{H}, \mathrm{NH}), 5.00-4.95(\mathrm{~m}, 1 \mathrm{H}, \mathrm{COCH}), 2.86-2.80$ $\left(\mathrm{m}, 1 \mathrm{H}, \mathrm{COCHCH}_{2}\right), 2.75-2.69\left(\mathrm{~m}, 1 \mathrm{H}, \mathrm{COCHCH}_{2}\right), 2.33\left(\mathrm{~s}, 3 \mathrm{H}, \mathrm{ArCH}_{3}\right), 2.19\left(\mathrm{~s}, 3 \mathrm{H}, \mathrm{ArCH}_{3}\right), 2.16-2.10(\mathrm{~m}$, 4H), 1.96-1.89 (m, 1H); $\left.{ }^{13} \mathrm{C} \mathrm{NMR} \mathrm{(125} \mathrm{MHz,} \mathrm{CDCl}_{3}\right) \delta 171.3,153.2,144.3,143.5,139.5,136.3,131.4$, $130.4,129.3,127.3,119.9,111.4,56.1,34.9,31.0,21.4,13.8,13.7$; IR (KBr) 3269, 2926, 1728, 1586, $1489,1484,1379,1358,1163,1090,961,816 \mathrm{~cm}^{-1}$; HRMS (ESI, H) $\mathrm{m} / \mathrm{z}$ calc'd for $\mathrm{C}_{22} \mathrm{H}_{25} \mathrm{BrN}_{3} \mathrm{O}_{3} \mathrm{~S}(\mathrm{M}+\mathrm{H})^{+}$ 490.0795 , found 490.0779 .

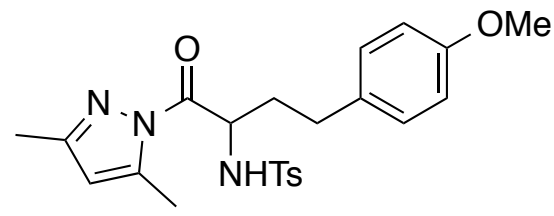

N-(1-(3,5-dimethyl-1H-pyrazol-1-yl)-4-(4-methoxyphenyl)-1-oxobutan-2-yl)-4

methylbenzenesulfonamide (3i) (white solid, 20\% EtOAc/ $n$-hexane, 69\% yield); ${ }^{1} \mathrm{H}-\mathrm{NMR}$ (500MHz, $\left.\mathrm{CDCl}_{3}\right)(\mathrm{ppm}) \delta 7.61(\mathrm{~d}, J=8.5 \mathrm{~Hz}, 2 \mathrm{H}, \mathrm{Ar}), 7.10(\mathrm{~d}, J=8.5 \mathrm{~Hz}, 2 \mathrm{H}, \mathrm{Ar}), 7.06(\mathrm{~d}, J=8.5 \mathrm{~Hz}, 2 \mathrm{H}, \mathrm{Ar}), 6.79(\mathrm{~d}, J$ $=8.5 \mathrm{~Hz}, 2 \mathrm{H}, \mathrm{Ar}), 5.85(\mathrm{~s}, 1 \mathrm{H}$, pyrazole), $5.73(\mathrm{~d}, \mathrm{~J}=10.5 \mathrm{~Hz}, 1 \mathrm{H}, \mathrm{NH}), 4.99(\mathrm{~m}, 1 \mathrm{H}, \mathrm{COCH}), 3.78(\mathrm{~s}, 3 \mathrm{H}$, $\left.\mathrm{OCH}_{3}\right), 2.84-2.78\left(\mathrm{~m}, 1 \mathrm{H}, \mathrm{C}(\mathrm{O}) \mathrm{CH}_{2} \mathrm{CH}_{2}\right), 2.70-2.64\left(\mathrm{~m}, 1 \mathrm{H}, \mathrm{COCHCH}_{2}\right), 2.33\left(\mathrm{~s}, 3 \mathrm{H}, \mathrm{ArCH}_{3}\right), 2.20(\mathrm{~s}, 3 \mathrm{H}$, $\left.\mathrm{ArCH}_{3}\right), 2.17-2.09(\mathrm{~m}, 4 \mathrm{H}), 1.97-1.90(\mathrm{~m}, 1 \mathrm{H}) ;{ }^{13} \mathrm{C} \mathrm{NMR}\left(125 \mathrm{MHz}, \mathrm{CDCl}_{3}\right) \delta$ 171.4, 158.0, 153.0, 144.3, 143.3, 136.5, 132.7, 129.4, 129.3, 127.3, 113.8, 111.3, 56.5, 55.3, 35.4, 30.7, 21.4, 13.8, 13.7; IR (KBr) $3271,2955,2928,1728,1586,1514,1379,1358,1331,1252,1161,1090,961 \mathrm{~cm}^{-1}$; HRMS (ESI, H) $\mathrm{m} / \mathrm{z}$ calc'd for $\mathrm{C}_{23} \mathrm{H}_{28} \mathrm{~N}_{3} \mathrm{O}_{4} \mathrm{~S}(\mathrm{M}+\mathrm{H})^{+} 442.1795$, found 442.1802 . 


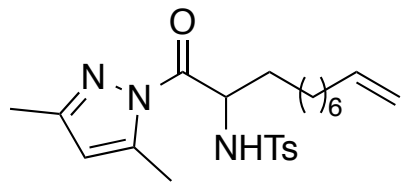

$N$-(1-(3,5-dimethyl-1H-pyrazol-1-yl)-1-oxoundec-10-en-2-yl)-4-methylbenzenesulfonamide (3j) (white solid, $16.7 \%$ EtOAc/ $n$-hexane, 58\% yield); ${ }^{1} \mathrm{H}-\mathrm{NMR}\left(500 \mathrm{MHz}, \mathrm{CDCl}_{3}\right)(\mathrm{ppm}) \delta 7.61(\mathrm{~d}, J=8.0 \mathrm{~Hz}, 2 \mathrm{H}, \mathrm{Ar})$, $7.10(\mathrm{~d}, J=8.0 \mathrm{~Hz}, 2 \mathrm{H}, \mathrm{Ar}), 5.87\left(\mathrm{~s}, 1 \mathrm{H}\right.$, pyrazole), 5.84-5.76 (m, $\left.1 \mathrm{H}, \mathrm{CHCH}_{2}\right), 5.62(\mathrm{~d}, J=11.0 \mathrm{~Hz}, 1 \mathrm{H}, \mathrm{NH})$, 5.01-4.91 (m, 3H, COCH, $\left.\mathrm{CHCH}_{2}\right), 2.32\left(\mathrm{~s}, 3 \mathrm{H}, \mathrm{ArCH}_{3}\right), 2.23\left(\mathrm{~s}, 3 \mathrm{H}, \mathrm{ArCH}_{3}\right), 2.18\left(\mathrm{~s}, 3 \mathrm{H}, \mathrm{ArCH}_{3}\right), 2.05-2.00$ (m, $2 \mathrm{H}, \mathrm{CH}_{2} \mathrm{CHCH}_{2}$ ), 1.85-1.26 (m, $12 \mathrm{H}$ overlapped with water); $\left.{ }^{13} \mathrm{C} \mathrm{NMR} \mathrm{(125} \mathrm{MHz,} \mathrm{CDCl}_{3}\right) \delta 171.9,152.9$, 144.3, 143.3, 139.2, 136.6, 129.2, 127.3, 114.2, 111.3, 56.5, 33.8, 33.5, 29.1, 29.0, 28.9, 28.7, 25.2, 21.4, 13.83, 13.82; IR (KBr) 3256, 2926, 2853, 1723, 1591, 1377, 1346, 1163, 961, $820 \mathrm{~cm}^{-1}$; HRMS (ESI, H) $m / z$ calc'd for $\mathrm{C}_{23} \mathrm{H}_{34} \mathrm{~N}_{3} \mathrm{O}_{3} \mathrm{~S}(\mathrm{M}+\mathrm{H})^{+} 432.2315$, found 432.2321 .

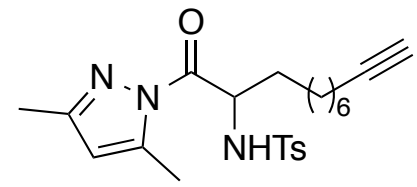

$N$-(1-(3,5-dimethyl-1H-pyrazol-1-yl)-1-oxoundec-10-yn-2-yl)-4-methylbenzenesulfonamide (3k) (white solid, 20\% EtOAc/n-hexane, 61\% yield); ${ }^{1} \mathrm{H}-\mathrm{NMR}\left(500 \mathrm{MHz}, \mathrm{CDCl}_{3}\right)(\mathrm{ppm}) \delta 7.61$ (d, J=8.5 Hz, $\left.2 \mathrm{H}, \mathrm{Ar}\right)$, $7.10(\mathrm{~d}, J=8.5 \mathrm{~Hz}, 2 \mathrm{H}, \mathrm{Ar}$ ), 5.87 (s, 1H, pyrazole), 5.61 (d, J = 11.0 Hz, 1H, NH), 5.00-4.95 (m, 1H, COCH), $2.33\left(\mathrm{~s}, 3 \mathrm{H}, \mathrm{ArCH}_{3}\right), 2.23\left(\mathrm{~s}, 3 \mathrm{H}, \mathrm{ArCH}_{3}\right), 2.18-2.15\left(\mathrm{~m}, 5 \mathrm{H}, \mathrm{ArCH}_{3} \mathrm{CH}_{2} \mathrm{CCH}\right), 1.93\left(\mathrm{t}, \mathrm{J}=3.0 \mathrm{~Hz}, 1 \mathrm{H}, \mathrm{CH}_{2} \mathrm{CCH}\right)$, 1.86-1.79 (m, 1H), 1.67-1.60 (m, 1H), 1.53-1.24 (m, 10H) ; $\left.{ }^{13} \mathrm{C} \mathrm{NMR} \mathrm{(125} \mathrm{MHz,} \mathrm{CDCl}_{3}\right) \delta$ 171.9, 152.9, 144.3, 143.3, 139.2, 136.6, 129.2, 127.3, 114.2, 111.3, 56.5, 33.8, 33.5, 29.1, 29.0, 28.9, 28.7, 25.2, 21.4, 13.83, 13.82; IR (KBr) 3279, 2930, 2857, 1732, 1377, 1356, 1167, 1153, 961, $818 \mathrm{~cm}^{-1}$; HRMS (ESI, H) m/z calc'd for $\mathrm{C}_{23} \mathrm{H}_{32} \mathrm{~N}_{3} \mathrm{O}_{3} \mathrm{~S}(\mathrm{M}+\mathrm{H})^{+} 430.2159$ found 430.2161 .

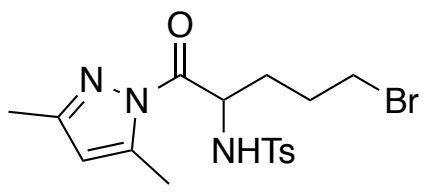

\section{N-(5-bromo-1-(3,5-dimethyl-1H-pyrazol-1-yl)-1-oxopentan-2-yl)-4-methylbenzenesulfonamide (3I)}

(white solid, 20\% EtOAc/ $n$-hexane, 73\% yield); ${ }^{1} 1 \mathrm{H}-\mathrm{NMR}\left(500 \mathrm{MHz}, \mathrm{CDCl}_{3}\right)(\mathrm{ppm}) \delta 7.61$ (d, $J=8.5 \mathrm{~Hz}, 2 \mathrm{H}$, Ar), $7.11(\mathrm{~d}, J=8.5 \mathrm{~Hz}, 2 \mathrm{H}, \mathrm{Ar}), 5.88(\mathrm{~s}, 1 \mathrm{H}$, pyrazole), $5.63(\mathrm{~d}, J=10.5,1 \mathrm{H}, \mathrm{NH}), 5.03-4.98(\mathrm{~m}, 1 \mathrm{H}, \mathrm{COCH})$, 
3.60-3.56 (m, $1 \mathrm{H}, \mathrm{CH}_{2} \mathrm{CH}_{2} \mathrm{Br}$ ), 3.46-3.44 (m, 1H, $\left.\mathrm{CH}_{2} \mathrm{CH}_{2} \mathrm{Br}\right), 2.33\left(\mathrm{~s}, 3 \mathrm{H}, \mathrm{ArCH}_{3}\right), 2.23\left(\mathrm{~s}, 3 \mathrm{H}, \mathrm{ArCH}_{3}\right), 2.19$ $\left(\mathrm{s}, 3 \mathrm{H}, \mathrm{ArCH}_{3}\right), 2.11-1.93(\mathrm{~m}, 3 \mathrm{H}), 1.79-1.72(\mathrm{~m}, 1 \mathrm{H}),{ }^{13} \mathrm{C} \mathrm{NMR}\left(125 \mathrm{MHz}, \mathrm{CDCl}_{3}\right) \delta 171.3,153.3,144.3$, 143.5, 136.3, 129.3, 127.3, 111.5, 55.8, 44.1, 32.6, 32.0, 30.8, 28.5, 21.4, 13.8 (overlapped); $\mathrm{IR} \mathrm{(KBr)}$ $3268,2926,1732,1584,1377,1352,1330,1300,1163,1090,959,816 \mathrm{~cm}^{-1} \mathrm{HRMS}$ (ESI, H) $\mathrm{m} / \mathrm{z}$ calc'd for $\mathrm{C}_{17} \mathrm{H}_{23} \mathrm{BrN}_{3} \mathrm{O}_{3} \mathrm{~S}(\mathrm{M}+\mathrm{H})^{+}$428.0638, found 428.0643 .

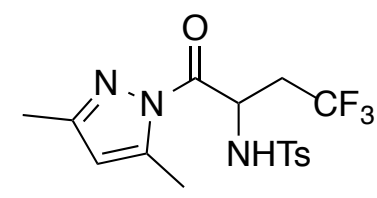

\section{$N$-(1-(3,5-dimethyl-1H-pyrazol-1-yl)-4,4,4-trifluoro-1-oxobutan-2-yl)-4-methylbenzenesulfonamide}

(3m) (white solid, 20\% EtOAc/n-hexane, 75\% yield); ${ }^{1} \mathrm{H}-\mathrm{NMR}\left(500 \mathrm{MHz}, \mathrm{CDCl}_{3}\right)(\mathrm{ppm}) \delta 7.66(\mathrm{~d}, J=8.0 \mathrm{~Hz}$, $2 \mathrm{H}, \mathrm{Ar}), 7.18(\mathrm{~d}, J=8.0 \mathrm{~Hz}, 2 \mathrm{H}, \mathrm{Ar}$ ), $5.92(\mathrm{~s}, 1 \mathrm{H}$, pyrazole), 5.81 (d, $J=10.5 \mathrm{~Hz}, 1 \mathrm{H}, \mathrm{NH}), 5.30-5.25(\mathrm{~m}, 1 \mathrm{H}$, $\mathrm{COCH}), 2.84-2.73\left(\mathrm{~m}, 2 \mathrm{H}, \mathrm{CH}_{2} \mathrm{CF}_{3}\right), 2.37\left(\mathrm{~s}, 3 \mathrm{H}, \mathrm{ArCH}_{3}\right), 2.26\left(\mathrm{~s}, 3 \mathrm{H}, \mathrm{ArCH}_{3}\right), 2.23\left(\mathrm{~s}, 3 \mathrm{H}, \mathrm{ArCH}_{3}\right) ;{ }^{13} \mathrm{CNMR}^{\mathrm{N}}$ $\left(125 \mathrm{MHz}, \mathrm{CDCl}_{3}\right) \delta 168.3,153.9,144.7,143.8,136.3,129.5,127.3,125.2(J=276 \mathrm{~Hz}), 111.8,51.8,37.6(J$ $=28.8 \mathrm{~Hz}), 21.5,13.9,13.7 ;{ }^{19} \mathrm{~F} \mathrm{NMR}\left(470 \mathrm{MHz}, \mathrm{CDCl}_{3}\right) \delta-63.0(J=10.3 \mathrm{~Hz}) ; \mathrm{IR}(\mathrm{KBr}) 3262,1734,1591$, $1379,1360,1335,1273,1159,1134,1038,963 \mathrm{~cm}^{-1}$; HRMS $(E S I, H) \mathrm{m} / z$ calc'd for $\mathrm{C}_{16} \mathrm{H}_{19} \mathrm{~F}_{3} \mathrm{~N}_{3} \mathrm{O}_{3} \mathrm{~S}(\mathrm{M}+\mathrm{H})^{+}$ 390.1094, found 390.1101 .

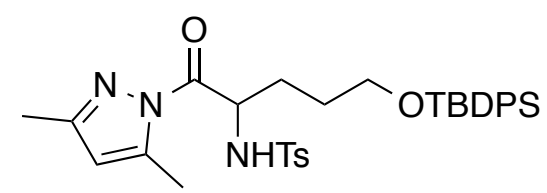

\section{$N$-(5-((tert-butyldiphenylsilyl)oxy)-1-(3,5-dimethyl-1H-pyrazol-1-yl)-1-oxopentan-2-yl)-4}

methylbenzenesulfonamide (3n) (colorless oil 14\%-50\% EtOAc/ $n$-hexane, 67\% yield) ; ${ }^{1} \mathrm{H}-\mathrm{NMR}(500$ $\left.\mathrm{MHz}, \mathrm{CDCl}_{3}\right)(\mathrm{ppm}) \delta$ 7.63-7.59 (m, 6H, Ar), 7.41-7.35 (m, 6H, Ar), 7.08 (d, J = 8.0 Hz, 2H, Ar), $5.87(\mathrm{~s}, 1 \mathrm{H}$, pyrazole), $5.66(\mathrm{~d}, J=10.5 \mathrm{~Hz}, 1 \mathrm{H}, \mathrm{NH}), 5.00-4.98(\mathrm{~m}, 1 \mathrm{H}, \mathrm{CHN}), 3.64\left(\mathrm{t}, J=6.0 \mathrm{~Hz}, 2 \mathrm{H}, \mathrm{OCH}_{2}\right), 2.32(\mathrm{~s}, 3 \mathrm{H}$, $\left.\mathrm{ArCH}_{3}\right), 2.20\left(\mathrm{~s}, 3 \mathrm{H}, \mathrm{ArCH}_{3}\right), 2.19\left(\mathrm{~m}, 3 \mathrm{H}, \mathrm{ArCH}_{3}\right), 2.03-1.98\left(\mathrm{~m}, 1 \mathrm{H}, \mathrm{CH}_{2}\right), 1.79-1.71\left(\mathrm{~m}, 2 \mathrm{H}, \mathrm{CH}_{2}\right), 1.64-1.61$ $\left(\mathrm{m}, 1 \mathrm{H}, \mathrm{CH}_{2}\right), 1.00\left(\mathrm{~s}, 9 \mathrm{H}, \mathrm{C}\left(\mathrm{CH}_{3}\right)_{3}\right) ;{ }^{13} \mathrm{C} \mathrm{NMR}\left(125 \mathrm{MHz}, \mathrm{CDCl}_{3}\right) \delta 171.6,153.0,144.3,143.3,136.5,135.5$, $133.8,129.6,129.2,127.6,127.3,111.3,62.9,56.6,30.1,28.5,26.8,21.4,19.2,13.83,13.81 ; \mathrm{IR}(\mathrm{KBr})$ 3246, 2928, 2857, 1724, 1587, 1427, 1377, 1348, 1165, 1111, 1044, 963, $904 \mathrm{~cm}^{-1} ; \mathrm{H} ;$ HRMS (ESI, H) m/z calc'd for $\mathrm{C}_{33} \mathrm{H}_{42} \mathrm{~N}_{3} \mathrm{O}_{4} \mathrm{SSi}(\mathrm{M}+\mathrm{H})^{+} 604.2660$, found 604.2667 


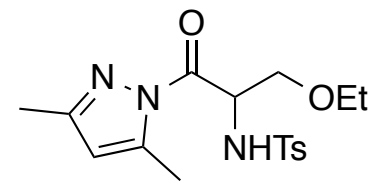

$N$-(1-(3,5-dimethyl-1H-pyrazol-1-yl)-3-ethoxy-1-oxopropan-2-yl)-4-methylbenzenesulfonamide (30) (white solid, 25\% EtOAc/n-hexane, 83\% yield); ${ }^{1} \mathrm{H}-\mathrm{NMR}\left(500 \mathrm{MHz}, \mathrm{CDCl}_{3}\right)(\mathrm{ppm}) \delta 7.67$ (d, $J=8.5 \mathrm{~Hz}, 2 \mathrm{H}$, Ar), $7.16(\mathrm{~d}, J=8.5 \mathrm{~Hz}, 2 \mathrm{H}, \mathrm{Ar}), 5.90(\mathrm{~s}, 1 \mathrm{H}$, pyrazole), 5.77 (d, $J=10.5 \mathrm{~Hz}, 1 \mathrm{H}, \mathrm{NH}), 5.29(\mathrm{~m}, 1 \mathrm{H}, \mathrm{COCH})$, 3.93-3.73 (m, 2H, CH $\mathrm{CH}_{2} \mathrm{OEt}$ ), 3.42 (q, J = $\left.7.0 \mathrm{~Hz}, 2 \mathrm{H}, \mathrm{OCH}_{2} \mathrm{CH}_{3}\right), 2.36\left(\mathrm{~s}, 3 \mathrm{H}, \mathrm{ArCH}_{3}\right), 2.28\left(\mathrm{~s}, 3 \mathrm{H}, \mathrm{ArCH}_{3}\right)$, $2.21\left(\mathrm{~s}, 3 \mathrm{H}, \mathrm{ArCH}_{3}\right), 1.06\left(\mathrm{t}, J=7.0 \mathrm{~Hz}, 3 \mathrm{H}, \mathrm{OCH}_{2} \mathrm{CH}_{3}\right) ;{ }^{13} \mathrm{CNMR}\left(125 \mathrm{MHz} \mathrm{CDCl}_{3}\right) \delta 168.9,152.9,144.4$, 143.4, 137.0, 129.4, 127.2, 111.3, 71.3, 66.9, 56.3, 21.4, 14.8, 13.82, 13.81; IR (KBr) 3275, 2976, 2928, $2868,1730,1587,1379,1356,1329,1165,1092,963,818 \mathrm{~cm}^{-1}$; HRMS (ESI, H) $\mathrm{m} / z$ calc'd for $\mathrm{C}_{17} \mathrm{H}_{24} \mathrm{~N}_{3} \mathrm{O}_{4} \mathrm{~S}$ $(\mathrm{M}+\mathrm{H})^{+} 366.1482$, found 366.1488

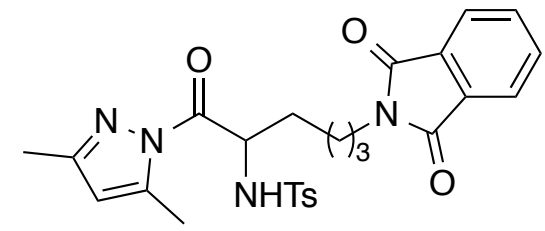

N-(1-(3,5-dimethyl-1H-pyrazol-1-yl)-6-(1,3-dioxoisoindolin-2-yl)-1-oxohexan-2-yl)-4-

methylbenzenesulfonamide (3p) (white solid, 33\% EtOAc/n-hexane, 65\% yield); ${ }^{1} \mathrm{H}-\mathrm{NMR}(500 \mathrm{MHz}$, $\left.\mathrm{CDCl}_{3}\right)(\mathrm{ppm}) \delta$ 7.85-7.83 (m, 2H, Ar), 7.72-7.70 (m, 2H, Ar), $7.61(\mathrm{~d}, J=8.5 \mathrm{~Hz}, 2 \mathrm{H}, \mathrm{Ar}), 7.10(\mathrm{~d}, J=8.5 \mathrm{~Hz}$, $2 \mathrm{H}, \operatorname{Ar}), 5.87(\mathrm{~s}, 1 \mathrm{H}$, pyrazole), $5.60(\mathrm{~d}, J=11.0 \mathrm{~Hz}, 1 \mathrm{H}, \mathrm{NH}), 5.02-4.97(\mathrm{~m}, 1 \mathrm{H}, \mathrm{COCH}), 3.66(\mathrm{t}, J=7.0 \mathrm{~Hz}$, $\left.2 \mathrm{H}, \mathrm{CH}_{2} \mathrm{~N}\right), 2.32\left(\mathrm{~s}, 3 \mathrm{H}, \mathrm{ArCH}_{3}\right), 2.22\left(\mathrm{~s}, 3 \mathrm{H}, \mathrm{ArCH}_{3}\right), 2.19\left(\mathrm{~s}, 3 \mathrm{H}, \mathrm{ArCH}_{3}\right), 1.93-1.86(\mathrm{~m}, 1 \mathrm{H}), 1.74-1.64(\mathrm{~m}$, 3H), 1.54-1.45 (m, $2 \mathrm{H}$ overlapped with water) ; ${ }^{13} \mathrm{C} \mathrm{NMR}\left(125 \mathrm{MHz}, \mathrm{CDCl}_{3}\right) \delta 171.6,168.4,153.1,144.3$, 143.3, 136.5, 133.9, 132.2, 129.2, 127.3, 123.2, 111.4, 56.2, 37.4, 32.8, 27.7, 22.5, 21.4, 13.84, 13.80; IR (KBr) 3273, 2928, 1775, 1732, 1705, 1402, 1360, 1300, 1163, 1090, 1053, $955 \mathrm{~cm}^{-1}$ HRMS (ESI, H) $\mathrm{m} / \mathrm{z}$ calc'd for $\mathrm{C}_{26} \mathrm{H}_{29} \mathrm{~N}_{4} \mathrm{O}_{5} \mathrm{~S}(\mathrm{M}+\mathrm{H})^{+} 509.1853$, found 509.1859 .

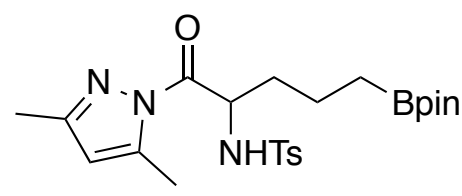

$N$-(1-(3,5-dimethyl-1H-pyrazol-1-yl)-1-oxo-5-(4,4,5,5-tetramethyl-1,3,2-dioxaborolan-2-yl)pentan-2-yl)- 
4-methylbenzenesulfonamide (3q) (white solid, 20\% EtOAc/n-hexane, 60\% yield); ${ }^{1} \mathrm{H}-\mathrm{NMR}(500 \mathrm{MHz}$, $\left.\mathrm{CDCl}_{3}\right)(\mathrm{ppm}) \delta 7.61(\mathrm{~d}, J=8.0 \mathrm{~Hz}, 2 \mathrm{H}, \mathrm{Ar}), 7.10(\mathrm{~d}, J=8.0 \mathrm{~Hz}, 2 \mathrm{H}, \mathrm{Ar}), 5.86(\mathrm{~s}, 1 \mathrm{H}$, pyrazole), 5.65, (d, $J=$ $11.0 \mathrm{~Hz}, 1 \mathrm{H}, \mathrm{NH})$, 5.01-4.96 (m, 1H, COCH), $2.32\left(\mathrm{~s}, 3 \mathrm{H}, \mathrm{ArCH}_{3}\right), 2.21\left(\mathrm{~s}, 3 \mathrm{H}, \mathrm{ArCH}_{3}\right), 2.19\left(\mathrm{~s}, 3 \mathrm{H}, \mathrm{ArCH}_{3}\right)$, 1.87-1.80 (m, 1H), 1.72-1.45 (m, 3H), $1.213\left(\mathrm{~s}, 6 \mathrm{H}, \mathrm{CH}_{3}\right), 1.210\left(\mathrm{~s}, 6 \mathrm{H}, \mathrm{CH}_{3}\right), 0.82-0.69\left(\mathrm{~m}, 2 \mathrm{H}, \mathrm{CH}_{2}\right) ;{ }^{13} \mathrm{C}$ NMR $\left(125 \mathrm{MHz}, \mathrm{CDCl}_{3}\right) \delta 171.7,152.8,144.2,143.2,136.6,129.2,127.3,111.2,83.0,56.6,35.9,24.8$, 24.8, 21.4, 19.9, 13.8 (overlapped); IR (KBr) 3244, 2980, 2923, 2874, 1728, 1589, 1375, 1356, 1325, 1165, 1146, 1092, 974, 959, $847 \mathrm{~cm}^{-1}$; HRMS (ESI, H) $\mathrm{m} / z$ calc'd for $\mathrm{C}_{23} \mathrm{H}_{35} \mathrm{BN}_{3} \mathrm{O}_{5} \mathrm{~S}(\mathrm{M}+\mathrm{H})^{+} 476.2385$, found 476.2389 .

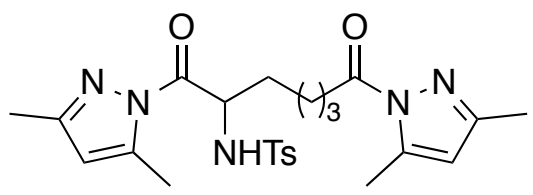

$N$-(1,7-bis(3,5-dimethyl-1H-pyrazol-1-yl)-1,7-dioxoheptan-2-yl)-4-methylbenzenesulfonamide (white solid, 14\% EtOAc/n-hexane, 60\% yield); ${ }^{1} \mathrm{H}-\mathrm{NMR}\left(500 \mathrm{MHz}, \mathrm{CDCl}_{3}\right)(\mathrm{ppm}) \delta 7.61$ (d, $J=8.5 \mathrm{~Hz}, 2 \mathrm{H}$, $\operatorname{Ar}-H), 7.10(\mathrm{~d}, J=8.5 \mathrm{~Hz}, 2 \mathrm{H}, \mathrm{Ar}-\mathrm{H}), 5.94(\mathrm{~s}, 1 \mathrm{H}$, pyrazole), 5.87 (s, 1H, pyrazole), 5.63 (d, $J=11 \mathrm{~Hz}, 1 \mathrm{H}$, $\mathrm{NH})$, 5.03-4.98 (m, $1 \mathrm{H}, \mathrm{C}(\mathrm{O}) \mathrm{CHNH}), 3.09-3.05\left(\mathrm{~m}, 2 \mathrm{H}, \mathrm{CH}_{2} \mathrm{CH}_{2} \mathrm{C}(\mathrm{O})\right), 2.53\left(\mathrm{~s}, 3 \mathrm{H}, \mathrm{ArCH}_{3}\right), 2.31(\mathrm{~s}, 3 \mathrm{H}$, $\left.\mathrm{ArCH}_{3}\right), 2.23\left(\mathrm{~s}, 3 \mathrm{H}, \mathrm{ArCH}_{3}\right), 2.22\left(\mathrm{~s}, 3 \mathrm{H}, \mathrm{ArCH}_{3}\right), 2.19\left(\mathrm{~s}, 3 \mathrm{H}, \mathrm{ArCH}_{3}\right), 1.94-1.87(\mathrm{~m}, 1 \mathrm{H}), 1.79-1.47(\mathrm{~m}, 5 \mathrm{H}$ overlapped with water) ; ${ }^{13} \mathrm{C}$ NMR $\left(125 \mathrm{MHz}, \mathrm{CDCl}_{3}\right) \delta$ 173.7, 171.7, 153.0, 151.8, 144.3, 143.0, 143.3, 136.5, 129.2, 127.3, 111.3, 111.0, 56.3, 34.8, 33.2, 24.8, 23.5, 21.3, 14.6 (overlapped), 13.82, 13.80; IR (KBr) 3258, 2942, 1728, 1591, 1582, 1412, 1392, 1362, 1165, 1390, 988, 963, $812 \mathrm{~cm}^{-1}$; HRMS (ESI, H) $m / z$ calc'd for $\mathrm{C}_{24} \mathrm{H}_{32} \mathrm{~N}_{5} \mathrm{O}_{4} \mathrm{~S}(\mathrm{M}+\mathrm{H})^{+} 486.2170$, found 486.2176 .

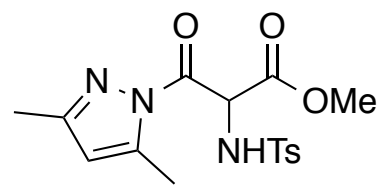

methyl 3-(3,5-dimethyl-1H-pyrazol-1-yl)-2-((4-methylphenyl)sulfonamido)-3-oxopropanoate (3s) (white solid, 33\% EtOAc/n-hexane, 70\% yield) ; ${ }^{1} \mathrm{H}-\mathrm{NMR}\left(500 \mathrm{MHz}, \mathrm{CDCl}_{3}\right)(\mathrm{ppm}) \delta 7.71(\mathrm{~d}, J=8.5 \mathrm{~Hz}, 2 \mathrm{H}$, Ar), $7.23(\mathrm{~d}, J=8.5 \mathrm{~Hz}, 2 \mathrm{H}, \mathrm{Ar}$ ), $6.02(\mathrm{~d}, J=9.5 \mathrm{~Hz}, 1 \mathrm{H}, \mathrm{COCH}$ ), 5.93 (s, 1H, pyrazole), 5.50 (d, J = $9.5 \mathrm{~Hz}$, $1 \mathrm{H}, \mathrm{NH}), 3.65\left(\mathrm{~s}, 3 \mathrm{H}, \mathrm{OCH}_{3}\right), 2.39\left(\mathrm{~s}, 3 \mathrm{H}, \mathrm{ArCH}_{3}\right), 2.36\left(\mathrm{~s}, 3 \mathrm{H}, \mathrm{ArCH}_{3}\right), 2.19\left(\mathrm{~s}, 3 \mathrm{H}, \mathrm{ArCH}_{3}\right) ;{ }^{13} \mathrm{C} \mathrm{NMR}(125$ $\left.\mathrm{MHz}, \mathrm{CDCl}_{3}\right) \delta 166.4,164.7,153.8,144.6,143.8,136.6,129.5,127.3,111.9,60.0,53.3,21.5,13.8,13.6$; 
IR (KBr) 3300, 2928, 1732, 1586, 1379, 1343, 1290, 1250, 1165, 1090, 1020, $820 \mathrm{~cm}^{-1}$; HRMS (ESI, H) $\mathrm{m} / \mathrm{z}$ calc'd for $\mathrm{C}_{16} \mathrm{H}_{20} \mathrm{~N}_{3} \mathrm{O}_{5} \mathrm{~S}(\mathrm{M}+\mathrm{H})^{+} 366.1118$, found 366.1126 .

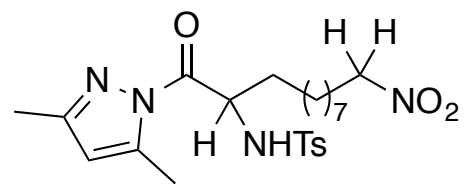

\section{$N$-(1-(3,5-dimethyl-1H-pyrazol-1-yl)-11-nitro-1-oxoundecan-2-yl)-4-methylbenzenesulfonamide (3t)}

(pale yellow solid, 20\% EtOAc/ $n$-hexane, $56 \%$ yield); ${ }^{1} \mathrm{H}-\mathrm{NMR}\left(500 \mathrm{MHz}, \mathrm{CDCl}_{3}\right)(\mathrm{ppm}) \delta 7.60(\mathrm{~d}, J=8.5 \mathrm{~Hz}$, $2 \mathrm{H}, \operatorname{Ar}$ ), 7.09 (d, J = 8.5 Hz, 2H, Ar), $5.87(\mathrm{~s}, 1 \mathrm{H}$, pyrazole), $5.63(\mathrm{~d}, J=11.0 \mathrm{~Hz}, 1 \mathrm{H}, \mathrm{NH}), 5.0-4.95(\mathrm{~m}, 1 \mathrm{H}$, $\mathrm{COCH}), 4.38\left(\mathrm{t}, J=7.0 \mathrm{~Hz}, 2 \mathrm{H}, \mathrm{CH}_{2} \mathrm{NO}_{2}\right), 2.32\left(\mathrm{~s}, 3 \mathrm{H}, \mathrm{ArCH}_{3}\right), 2.22\left(\mathrm{~s}, 3 \mathrm{H}, \mathrm{ArCH}_{3}\right), 2.18\left(\mathrm{~s}, 3 \mathrm{H}, \operatorname{ArCH}_{3}\right), 2.00$ (quin, $\left.J=7.0 \mathrm{~Hz}, 2 \mathrm{H}, \mathrm{CH}_{2} \mathrm{CH}_{2} \mathrm{NO}_{2}\right), 1.86-1.79(\mathrm{~m}, 1 \mathrm{H}), 1.67-1.60(\mathrm{~m}, 1 \mathrm{H}), 1.50-1.43(\mathrm{~m}, 2 \mathrm{H}), 1.40-1.26(\mathrm{~m}$, $10 \mathrm{H}) ;{ }^{13} \mathrm{CNMR}\left(125 \mathrm{MHz}, \mathrm{CDCl}_{3}\right) \delta 171.8,152.9,144.3,143.3,136.5,129.2,127.3,111.3,75.7,56.5$, 33.5, 29.068, 29.055, 28.8, 28.6, 27.4, 26.2, 25.2, 21.4, 13.84, 13.80; IR (KBr) 3266, 2924, 2855, 1736, $1584,1549,1377,1356,1333,1163,1092,959 \mathrm{~cm}^{-1}$; HRMS (ESI, H) $\mathrm{m} / z$ calc'd for $\mathrm{C}_{23} \mathrm{H}_{35} \mathrm{~N}_{4} \mathrm{O}_{5} \mathrm{~S}(\mathrm{M}+\mathrm{H})^{+}$ 479.2323, found 479.2326 .

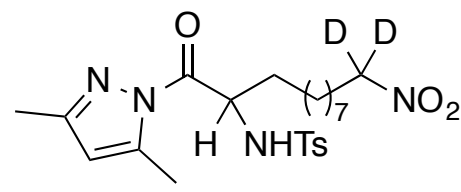

\section{$N$-(1-(3,5-dimethyl-1H-pyrazol-1-yl)-11-nitro-1-oxoundecan-2-yl-11,11- $\left.d_{2}\right)-4$}

methylbenzenesulfonamide $\left(\mathbf{3 t}\left(d^{2}\right)\right)$ (pale yellow solid, 20\% EtOAc/n-hexane, $56 \%$ yield)); ${ }^{1} \mathrm{H}-\mathrm{NMR}$ $\left(500 \mathrm{MHz}, \mathrm{CDCl}_{3}\right)(\mathrm{ppm}) \delta 7.60$ (d, $\left.J=8.0 \mathrm{~Hz}, 2 \mathrm{H}, \mathrm{Ar}\right), 7.09$ (d, $J=8.0 \mathrm{~Hz}, 2 \mathrm{H}, \mathrm{Ar}$ ), 5.87 (s, $1 \mathrm{H}$, pyrazole), $5.61(\mathrm{~d}, J=10.5 \mathrm{~Hz}, 1 \mathrm{H}, \mathrm{NH}), 5.0-4.95(\mathrm{~m}, 1 \mathrm{H}, \mathrm{COCH}), 2.32\left(\mathrm{~s}, 3 \mathrm{H}, \mathrm{ArCH}_{3}\right), 2.22\left(\mathrm{~s}, 3 \mathrm{H}, \mathrm{ArCH}_{3}\right), 2.17(\mathrm{~s}, 3 \mathrm{H}$, $\left.\mathrm{ArCH}_{3}\right), 1.99\left(\mathrm{t}, \mathrm{J}=7.0 \mathrm{~Hz}, 2 \mathrm{H}, \mathrm{CH}_{2} \mathrm{CD}_{2} \mathrm{NO}_{2}\right), 1.86-1.79(\mathrm{~m}, 1 \mathrm{H}), 1.67-1.60(\mathrm{~m}, 1 \mathrm{H}), 1.48-1.26(\mathrm{~m}, 12 \mathrm{H}) ;{ }^{13} \mathrm{C}$ $\operatorname{NMR}\left(125 \mathrm{MHz}, \mathrm{CDCl}_{3}\right) \delta 171.8,152.9,144.3,143.3,136.5,129.2,127.3,111.3,75.1(\mathrm{~J}=22.0 \mathrm{~Hz}), 56.5$, $33.5,29.068,29.056,28,8,28,6,27.2,26.1,25.2$, 21.4, 13.839, 13.803; IR (KBr) 3015, 2930, 2859, 1717, $1541,1379,1358,1165,1092,961 \mathrm{~cm}^{-1}$; HRMS (ESI, H) $\mathrm{m} / z$ calc'd for $\mathrm{C}_{23} \mathrm{H}_{33} \mathrm{D}_{2} \mathrm{~N}_{4} \mathrm{O}_{5} \mathrm{~S}(\mathrm{M}+\mathrm{H})^{+} 481.2448$, found 481.2453 . 
<smiles>CC(=O)c1ccc(CCC(NS)C(=O)n2nc(C)cc2C)cc1</smiles>

N-(4-(4-acetylphenyl)-1-(3,5-dimethyl-1H-pyrazol-1-yl)-1-oxobutan-2-yl)-4-methylbenzenesulfonamide (3u) (pale yellow solid, 25\%-40\% EtOAc/n-hexane, 70\% yield) ; ${ }^{1} \mathrm{H}-\mathrm{NMR}\left(500 \mathrm{MHz}, \mathrm{CDCl}_{3}\right.$ ) (ppm) $\delta 7.86$ (d, $J=8.5 \mathrm{~Hz}, 2 \mathrm{H}, \operatorname{Ar}$ ), $7.60(\mathrm{~d}, J=8.5 \mathrm{~Hz}, 2 \mathrm{H}, \operatorname{Ar}), 7.27$ (d, J = 8.0 Hz, 2H, Ar), 7.10 (d, J = 8.0 Hz, 2H, Ar), 5.88 (s, 1H, pyrazole), $5.76(\mathrm{~d}, J=11.0 \mathrm{~Hz}, 1 \mathrm{H}, \mathrm{NH})$, 5.03-4.98 $(\mathrm{m}, 1 \mathrm{H}, \mathrm{CHN}), 2.96-2.92(\mathrm{~m}, 1 \mathrm{H})$, 2.87-2.82 (m, $1 \mathrm{H}), 2.58\left(\mathrm{~s}, 3 \mathrm{H}, \mathrm{COCH}_{3}\right), 2.32\left(\mathrm{~s}, 3 \mathrm{H}, \mathrm{ArCH}_{3}\right), 2.24-2.15(\mathrm{~m}, 4 \mathrm{H}), 2.15\left(\mathrm{~s}, 3 \mathrm{H}, \mathrm{ArCH}_{3}\right), 2.00-1.93(\mathrm{~m}, 1 \mathrm{H}$, $\left.\mathrm{CH}_{2}\right) ;{ }^{13} \mathrm{C}$ NMR $\left(125 \mathrm{MHz}, \mathrm{CDCl}_{3}\right) \delta 197.8,171.2,153.2,146.4,144.3,143.5,136.3,135.3,129.3$, 128.8 ,128.5, 127.3, 111.4, 56.2, 34.6, 31.6, 26.6, 21.4, 13.8, 13.7.; IR (KBr) 3262, 1730, 1707, 1682, 1379, 1360, 1335, 1269, cm ${ }^{-1} ; \mathrm{H}$; HRMS $(E S I, H) ~ m / z$ calc'd for $\mathrm{C}_{24} \mathrm{H}_{28} \mathrm{~N}_{3} \mathrm{O} 4 \mathrm{~S}(\mathrm{M}+\mathrm{H})^{+}$454.1795, found 454.1804 .<smiles>CCC(=O)c1ccc(CCC(N)C(=O)n2nc(C)cc2C)cc1</smiles>

\section{$N$-(1-(3,5-dimethyl-1H-pyrazol-1-yl)-1-oxo-4-(4-propionylphenyl)butan-2-yl)-4-}

methylbenzenesulfonamide (3v) (white solid, 25\%-33\% EtOAc/ $n$-hexane, $72 \%$ yield) ; ${ }^{1} \mathrm{H}-\mathrm{NMR}$ (500 MHz, $\left.\mathrm{CDCl}_{3}\right)(\mathrm{ppm}) \delta 7.87(\mathrm{~d}, J=8.5 \mathrm{~Hz}, 2 \mathrm{H}, \mathrm{Ar}), 7.61(\mathrm{~d}, J=8.5 \mathrm{~Hz}, 2 \mathrm{H}, \mathrm{Ar}), 7.26(\mathrm{~d}, J=8.5 \mathrm{~Hz}, 2 \mathrm{H}, \mathrm{Ar}), 7.10(\mathrm{~d}, J$ $=8.5 \mathrm{~Hz}, 2 \mathrm{H}, \mathrm{Ar}), 5.86(\mathrm{~s}, 1 \mathrm{H}$, pyrazole), $5.76(\mathrm{~d}, J=10.5 \mathrm{~Hz}, 1 \mathrm{H}, \mathrm{NH}), 5.03-4.98(\mathrm{~m}, 1 \mathrm{H}, \mathrm{CHN}), 3.00-2.92$ $(\mathrm{m}, 3 \mathrm{H}), 2.86-2.80(\mathrm{~m}, 1 \mathrm{H}), 2.34\left(\mathrm{~s}, 3 \mathrm{H}, \mathrm{ArCH}_{3}\right), 2.20-2.15(\mathrm{~m}, 7 \mathrm{H}), 2.00-1.95(\mathrm{~m}, 1 \mathrm{H}), 1.22(\mathrm{t}, J=7.0 \mathrm{~Hz}$, $\left.3 \mathrm{H}, \mathrm{CH}_{3}\right) ;{ }^{13} \mathrm{C}$ NMR $\left(125 \mathrm{MHz}, \mathrm{CDCl}_{3}\right) \delta 200.5,171.2,153.2,146.1,144.3,143.5,136.3,135.0,129.3$, $128.8,128.2,127.3,111.4,56.2,34.6,31.7,31.6,21.4,13.8,13.7,8.31 ; \operatorname{IR}(\mathrm{KBr}) 3275,2928,1730,1684$, 1697, 1377, 1358, 1161, 1090, $959 \mathrm{~cm}^{-1} ; \mathrm{H}$; HRMS (ESI, H) $\mathrm{m} / z$ calc'd for $\mathrm{C}_{25} \mathrm{H}_{30} \mathrm{~N}_{3} \mathrm{O}_{4} \mathrm{~S}(\mathrm{M}+\mathrm{H})^{+} 468.1952$, found 468.1960 . 
<smiles>CC(=O)CC(N)C(=O)n1nc(C)cc1C</smiles>

$N$-(1-(3,5-dimethyl-1H-pyrazol-1-yl)-1,4-dioxopentan-2-yl)-4-methylbenzenesulfonamide (3w) (white solid, 25\%-50\% EtOAc/ $n$-hexane, $45 \%$ yield) ; ${ }^{1} \mathrm{H}-\mathrm{NMR}\left(500 \mathrm{MHz}, \mathrm{CDCl}_{3}\right)(\mathrm{ppm}) \delta 7.65$ (d, $J=8.5 \mathrm{~Hz}, 2 \mathrm{H}$, Ar), $7.18(\mathrm{~d}, J=8.5 \mathrm{~Hz}, 2 \mathrm{H}, \mathrm{Ar}$ ), $5.92(\mathrm{~d}, J=9.0 \mathrm{~Hz}, 1 \mathrm{H}, \mathrm{NH}), 5.88(\mathrm{~s}, 1 \mathrm{H}$, pyrazole), 5.23-5.18 (m, $1 \mathrm{H}, \mathrm{CHN})$, $3.16\left(\mathrm{~d}, J=5.5 \mathrm{~Hz}, 2 \mathrm{H}, \mathrm{CH}_{2} \mathrm{CO}\right), 2.36(\mathrm{~s}, 3 \mathrm{H}), 2.27(\mathrm{~s}, 3 \mathrm{H}), 2.20(\mathrm{~s}, 3 \mathrm{H}), 2.15(\mathrm{~s}, 3 \mathrm{H}) ;{ }^{13} \mathrm{C} \mathrm{NMR}(125 \mathrm{MHz}$, $\left.\mathrm{CDCl}_{3}\right) \delta 204.8,169.3,153.1,144.7,143.6,136.4,129.4,127.3,111.3,52.9,46.7,30.0,21.5,13.82$, 13.77; IR (KBr) 3279, 1732, 1587, 1379, 1358, 1302, 1159, 1092, $961 \mathrm{~cm}^{-1}$; HRMS (ESI, H) $\mathrm{m} / \mathrm{z}$ calc'd for $\mathrm{C}_{17} \mathrm{H}_{22} \mathrm{~N}_{3} \mathrm{O}_{4} \mathrm{~S}(\mathrm{M}+\mathrm{H})^{+} 364.1326$, found 364.1332 .<smiles>CCOC(=O)CCCCC(N)C(=O)n1nc(C)cc1C</smiles>

ethyl 7-(3,5-dimethyl-1H-pyrazol-1-yl)-6-((4-methylphenyl)sulfonamido)-7-oxoheptanoate (3x) (white solid, 20\%-33\% EtOAc/n-hexane, 72\% yield) ; ${ }^{1} \mathrm{H}-\mathrm{NMR}\left(500 \mathrm{MHz}, \mathrm{CDCl}_{3}\right)(\mathrm{ppm}) \delta 7.60(\mathrm{~d}, J=8.5 \mathrm{~Hz}, 2 \mathrm{H}$, $\operatorname{Ar}), 7.10(\mathrm{~d}, J=8.5 \mathrm{~Hz}, 2 \mathrm{H}, \operatorname{Ar}), 5.87(\mathrm{~s}, 1 \mathrm{H}$, pyrazole), $5.60(\mathrm{~d}, J=11.0 \mathrm{~Hz}, 1 \mathrm{H}, \mathrm{NH}), 5.00-4.95(\mathrm{~m}, 1 \mathrm{H}$, $\mathrm{CHN}), 4.12\left(\mathrm{q}, J=7.0 \mathrm{~Hz}, 2 \mathrm{H}, \mathrm{OCH}_{2}\right), 2.32\left(\mathrm{~s}, 3 \mathrm{H}, \mathrm{ArCH}_{3}\right), 2.28\left(\mathrm{dt}, J=7.5,2.0 \mathrm{~Hz}, 2 \mathrm{H}, \mathrm{COCH}_{2}\right), 2.22(\mathrm{~s}, 3 \mathrm{H}$, $\left.\mathrm{ArCH}_{3}\right), 2.18\left(\mathrm{~s}, 3 \mathrm{H}, \mathrm{ArCH}_{3}\right), 1.89-1.82(\mathrm{~m}, 1 \mathrm{H}), 1.69-1.60(\mathrm{~m}, 3 \mathrm{H}), 1.59-1.44(\mathrm{~m}, 2 \mathrm{H}$, overlapped with $\left.\mathrm{H}_{2} \mathrm{O}\right), 1.25\left(\mathrm{t}, J=7.0 \mathrm{~Hz}, 3 \mathrm{H}, \mathrm{OCH}_{2} \mathrm{CH}_{3}\right) ;{ }^{13} \mathrm{C} \mathrm{NMR}\left(125 \mathrm{MHz}, \mathrm{CDCl}_{3}\right) \delta 173.5,171.6,153.0,144.2,143.3$, $136.4,129.2,127.2,111.3,60.2,56.2,33.9,33.1,24.8,24.1,21.3,14.2,13.80,13.76$; IR (KBr) 3270, $3242,2984,2938,1732,1586,1445,1377,1348,1290,1219,1091,1032,962 \mathrm{~cm}^{-1}$; HRMS (ESI, H) m/z calc'd for $\mathrm{C}_{21} \mathrm{H}_{30} \mathrm{~N}_{3} \mathrm{O}_{5} \mathrm{~S}(\mathrm{M}+\mathrm{H})^{+} 436.1901$, found 436.1906.<smiles>Cc1cc(C)n(C(=O)C(N)CCC#N)n1</smiles>

\section{$N$-(4-cyano-1-(3,5-dimethyl-1H-pyrazol-1-yl)-1-oxobutan-2-yl)-4-methylbenzenesulfonamide} (white solid, 25\%-50\% EtOAc/n-hexane, 75\% yield) ; ${ }^{1} \mathrm{H}-\mathrm{NMR}\left(500 \mathrm{MHz}, \mathrm{CDCl}_{3}\right)(\mathrm{ppm}) \delta 7.62(\mathrm{~d}, J=8.0 \mathrm{~Hz}$, $2 \mathrm{H}, \mathrm{Ar}$ ), $7.14(\mathrm{~d}, J=8.0 \mathrm{~Hz}, 2 \mathrm{H}, \mathrm{Ar}$ ), $5.92(\mathrm{~s}, 1 \mathrm{H}, \mathrm{pyrazole}), 5.70(\mathrm{~d}, J=10.5 \mathrm{~Hz}, 1 \mathrm{H}, \mathrm{NH}$ ), 5.00 (dt, J = 10.0, 
$4.0 \mathrm{~Hz}, 1 \mathrm{H}, \mathrm{CHN}), 2.69-2.62\left(\mathrm{~m}, 1 \mathrm{H}, \mathrm{CH}_{2}\right), 2.58-2.52\left(\mathrm{~m}, 1 \mathrm{H}, \mathrm{CH}_{2}\right), 2.34\left(\mathrm{~s}, 3 \mathrm{H}, \mathrm{ArCH}_{3}\right), 2.33-2.27(\mathrm{~m}, 1 \mathrm{H}$, $\mathrm{CH}_{2}$ ), $2.24\left(\mathrm{~s}, 3 \mathrm{H}, \mathrm{ArCH}_{3}\right), 2.22\left(\mathrm{~s}, 3 \mathrm{H}, \mathrm{ArCH}_{3}\right), 2.01-1.94\left(\mathrm{~m}, 1 \mathrm{H}, \mathrm{CH}_{2}\right) ;{ }^{13} \mathrm{C} \mathrm{NMR}\left(125 \mathrm{MHz}, \mathrm{CDCl}_{3}\right) \delta 169.8$, 154.0, 144.6, 143.9, 135.9, 129.5, 127.3, 118.5, 111.9, 55.5, 29.6, 21.4, 13.93, 13.85, 13.7; IR (KBr) 3264, $1732,1589,1381,1358,1331,1163,1090,961 \mathrm{~cm}^{-1}$; HRMS (ESI, Na) $\mathrm{m} / z$ calc'd for $\mathrm{C}_{17} \mathrm{H}_{21} \mathrm{~N}_{4} \mathrm{O}_{3} \mathrm{~S}(\mathrm{M}+\mathrm{H})^{+}$ 361.1329 , found 361.1332 .

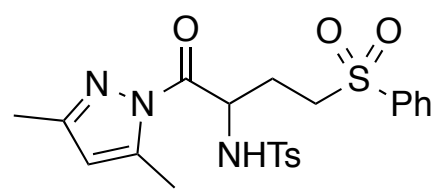

\section{$N$-(1-(3,5-dimethyl-1H-pyrazol-1-yl)-1-oxo-4-(phenylsulfonyl)butan-2-yl)-4-methylbenzenesulfonamide}

(3z) (white solid, 40\% EtOAc/n-hexane, 71\% yield); ${ }^{1} \mathrm{H}-\mathrm{NMR}\left(500 \mathrm{MHz}, \mathrm{CDCl}_{3}\right)(\mathrm{ppm}) \delta 7.92-7.90(\mathrm{~m}, 2 \mathrm{H}$, Ar), $7.70(\mathrm{~m}, 1 \mathrm{H}, \mathrm{Ar}), 7.60-7.55(\mathrm{~m}, 4 \mathrm{H}), 7.09(\mathrm{~d}, J=8.0 \mathrm{~Hz}, 2 \mathrm{H}, \mathrm{Ar}), 5.89(\mathrm{~s}, 1 \mathrm{H}$, pyrazole), $5.60(\mathrm{~d}, J=10.5$ $\mathrm{Hz}, 1 \mathrm{H}, \mathrm{NH}), 4.99(\mathrm{~m}, 1 \mathrm{H}, \mathrm{COCH}), 3.45-3.39(\mathrm{~m}, 1 \mathrm{H}), 3.28-3.21(\mathrm{~m}, 1 \mathrm{H}), 2.42(\mathrm{~m}, 1 \mathrm{H}), 2.32\left(\mathrm{~s}, 3 \mathrm{H}, \mathrm{ArCH}_{3}\right)$, $\left.2.21\left(\mathrm{~s}, 3 \mathrm{H}, \mathrm{ArCH}_{3}\right), 2.18\left(\mathrm{~s}, 3 \mathrm{H}, \mathrm{ArCH}_{3}\right), 2.07-1.99(\mathrm{~m}, 1 \mathrm{H}) ;{ }^{13} \mathrm{C} \mathrm{NMR} \mathrm{(125} \mathrm{MHz,} \mathrm{CDCl}\right) \delta 170.0,153.8$, 144.5, 143.8, 138.9, 135.9, 133.9, 129.4 (overlapped), 128.1, 127.2, 111.9, 55.1, 52.8, 26.5, 21.4, 13.9, 13.7; IR (KBr) 3268, 2926, 1728, 1587, 1379, 1358, 1310, 1161, 1086, $961 \mathrm{~cm}^{-1}$; HRMS (ESI, H) m/z calc'd for $\mathrm{C}_{22} \mathrm{H}_{26} \mathrm{~N}_{3} \mathrm{O}_{5} \mathrm{~S}_{2}(\mathrm{M}+\mathrm{H})^{+} 476.1308$, found 476.1311.

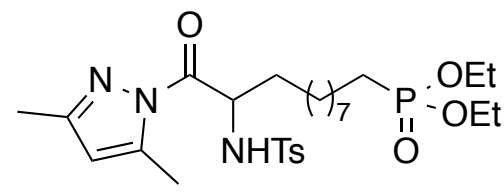

diethyl

(11-(3,5-dimethyl-1H-pyrazol-1-yl)-10-((4-methylphenyl)sulfonamido)-11-

oxoundecyl)phosphonate $(\mathbf{3} \boldsymbol{\alpha})$ (pale yellow solid, 33\% Acetone/ $n$-hexane, 33\% yield) ; ${ }^{1} \mathrm{H}-\mathrm{NMR}$ (500 $\mathrm{MHz}, \mathrm{CDCl}_{3}$ ) (ppm) $\delta 7.60$ (d, J = 7.9 Hz, 2H, Ar), 7.09 (d, J = $7.9 \mathrm{~Hz}, 2 \mathrm{H}, \mathrm{Ar}$ ), 5.87 (s, 1H, pyrazole), 5.69 (d, $J=11.0 \mathrm{~Hz}, 1 \mathrm{H}, \mathrm{NH}), 5.01-4.97(\mathrm{~m}, 1 \mathrm{H}, \mathrm{COCH}), 4.13-4.05\left(\mathrm{~m}, 4 \mathrm{H}, \mathrm{OCH}_{2}\right), 2.32\left(\mathrm{~s}, 3 \mathrm{H}, \mathrm{ArCH}_{3}\right), 2.22(\mathrm{~s}, 3 \mathrm{H}$, $\left.\mathrm{ArCH}_{3}\right), 2.18\left(\mathrm{~s}, 3 \mathrm{H}, \mathrm{ArCH}_{3}\right), 1.85-1.25\left(\mathrm{~m}, 24 \mathrm{H}\right.$, overlapped with water); ${ }^{13} \mathrm{C} \mathrm{NMR}\left(125 \mathrm{MHz}, \mathrm{CDCl}_{3}\right) \delta$ $170.8,151.9,143.2,142.2,135.5,128.17,128.21,110.3,60.4(J=6.45 \mathrm{~Hz}), 55.4,32.4,29.6(J=67.9)$, $28.2(J=4.1 \mathrm{~Hz}), 28.0,27.7,25.2,24.2,24.1,21.4(J=5.24 \mathrm{~Hz}), 20.4,15.5(J=6.13 \mathrm{~Hz}), 12.81,12.79 ;{ }^{31} \mathrm{P}$ NMR $\left(200 \mathrm{MHz}_{\mathrm{CDCl}}\right.$ ) $\delta$ 32.6; IR (thin film, NaCl) 2982, 2928, 2855, 1732, 1588, 1379, 1356, 1227, 1163, 1094, 1028, $961 \mathrm{~cm}^{-1}$; HRMS (ESI, H) $\mathrm{m} / z$ calc'd for $\mathrm{C}_{27} \mathrm{H}_{45} \mathrm{~N}_{3} \mathrm{O}_{6} \mathrm{P}(\mathrm{M}+\mathrm{H})^{+} 570.2761$, found 570.2760 


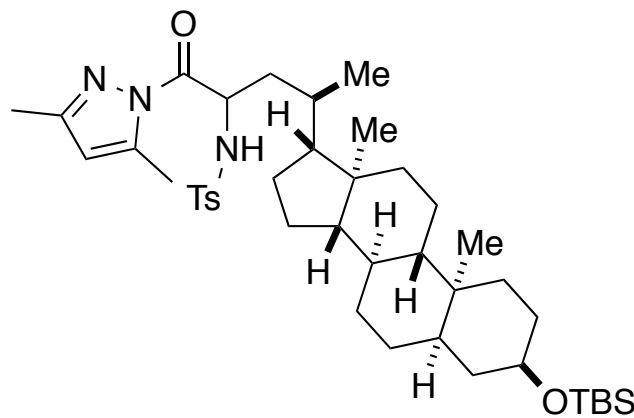

$N-((4 R)-4-((3 R, 5 R, 8 R, 9 S, 10 S, 13 R, 14 S, 17 R)-3-((t e r t-b u t y l d i m e t h y l s i l y l) 0 x y)-10,13-$

dimethylhexadecahydro-1H-cyclopenta[a]phenanthren-17-yl)-1-(3,5-dimethyl-1H-pyrazol-1-yl)-1-

oxopentan-2-yl)-4-methylbenzenesulfonamide (3ß) (diastereomixture) (white solid, $13 \% \mathrm{EtOAc} / n$ hexane, 54\% yield) ; ${ }^{1} \mathrm{H}-\mathrm{NMR}\left(500 \mathrm{MHz}, \mathrm{CDCl}_{3}\right)(\mathrm{ppm}) \delta$ 7.63-7.59 (m, 4H, Ar, diastereomers), 7.11-7.08 ( $\mathrm{m}, 4 \mathrm{H}, \mathrm{Ar}$, diastereomers), 5.87-5.85 (m, 2H, pyrazole, diastereomers), $5.76(\mathrm{~d}, J=11.0 \mathrm{~Hz}, 1 \mathrm{H}, \mathrm{NH})$, 5.48-5.40 (m, 1H, NH), 5.12-5.08 (m, 1H, CHN), 4.97-4.91 (m, 1H, CHN), 3.60-3.54 (m, 2H, diastereomers), $2.33\left(\mathrm{~s}, 3 \mathrm{H}, \mathrm{ArCH}_{3}\right), 2.32\left(\mathrm{~s}, 3 \mathrm{H}, \mathrm{ArCH}_{3}\right), 2.22$ (s, 6H, $\mathrm{ArCH}_{3}$, diastereomers), 2.17 (s, 6H, $\mathrm{ArCH}_{3}$, diastereomers), 1.98-1.72 (m, 12H, diastereomers), 1.57-0.88 (m, $70 \mathrm{H}$, diastereomers), $0.64\left(\mathrm{~s}, 3 \mathrm{H}, \mathrm{CH}_{3}\right)$, $0.54\left(\mathrm{~s}, 3 \mathrm{H}, \mathrm{CH}_{3}\right), 0.05$ (s, 12H, Si $\left(\mathrm{CH}_{3}\right)_{2}$, diastereomers) ; ${ }^{13} \mathrm{CNMR}\left(125 \mathrm{MHz}, \mathrm{CDCl}_{3}\right) \delta 172.6,171.8,152.9$, 152.7, 144.23, 144.20, 143.3, 143.2, 136.6, 136.5, 129.2, 127.4, 127.3, 111.2, 111.1, 72.9, 72.8, 56.5, 56.4, 56.30, 56.28, 43.0, 42.8, 42.3, 40.7, 40.2, 40.1, 40.0, 39.7, 36.9, 35.8, 35.60, 35.58, 34.60, 34.58, $34.0,32.9,31.0,29.7,28.4,28.2$, 27.3, 26.4, 26.0, 24.2, 23.4, 21.39, 21.37, 20.82, 20.76, 19.7, 18.4, 18.3, $17.7,13.9,13.80,13.76,12.1,11.8,-4.59,-4.60$; IR (neat) 3838, 3750, 2930, 2857, 1717, 1456, 1377, 1356, 1165, 1091, $961 \mathrm{~cm}^{-1}$; HRMS (ESI, H) $\mathrm{m} / z$ calc'd for $\mathrm{C}_{42} \mathrm{H}_{68} \mathrm{~N}_{3} \mathrm{O}_{4} \mathrm{SSi}_{2}(\mathrm{M}+\mathrm{H})^{+} 738.4694$, found 738.4693.

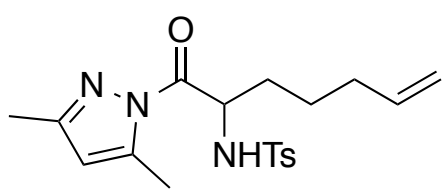

$\mathrm{N}$-(1-(3,5-dimethyl-1H-pyrazol-1-yl)-1-(|3-oxidanylidene)hept-6-en-2-yl)-4-methylbenzenesulfonamide (3y) (white solid, 16.7\% EtOAc/n-hexane, 58\% yield); ${ }^{1} \mathrm{H}-\mathrm{NMR}\left(500 \mathrm{MHz}, \mathrm{CDCl}_{3}\right)(\mathrm{ppm}) \delta 7.61(\mathrm{~d}, J=8.0 \mathrm{~Hz}$, $2 \mathrm{H}, \mathrm{Ar}), 7.10(\mathrm{~d}, J=8.0 \mathrm{~Hz}, 2 \mathrm{H}, \mathrm{Ar}), 5.87\left(\mathrm{~s}, 1 \mathrm{H}\right.$, pyrazole), 5.79-5.71 (m, $\left.1 \mathrm{H}, \mathrm{CHCH}_{2}\right), 5.62(\mathrm{~d}, J=11.0 \mathrm{~Hz}$, $1 \mathrm{H}, \mathrm{NH}), 5.02-4.92\left(\mathrm{~m}, 3 \mathrm{H}, \mathrm{COCH}, \mathrm{CHCH}_{2}\right), 2.32\left(\mathrm{~s}, 3 \mathrm{H}, \mathrm{ArCH}_{3}\right), 2.22\left(\mathrm{~s}, 3 \mathrm{H}, \mathrm{ArCH}_{3}\right), 2.18\left(\mathrm{~s}, 3 \mathrm{H}, \mathrm{ArCH}_{3}\right)$, 2.10-2.02 (m, $\left.2 \mathrm{H}, \mathrm{CH}_{2} \mathrm{CHCH}_{2}\right), 1.88-1.81(\mathrm{~m}, 1 \mathrm{H}), 1.69-1.49\left(\mathrm{~m}, 8 \mathrm{H}\right.$, overlapped with water) ; ${ }^{13} \mathrm{C}$ NMR 
$\left(125 \mathrm{MHz}, \mathrm{CDCl}_{3}\right) \delta 171.7,153.0,144.3,143.3,138.0,136.5,129.2,127.3,115.0,111.3,56.3,32.9,32.8$,

24.5, 21.4, 13.82, 13.80; IR (KBr) cm ${ }^{-1}$; $\operatorname{HRMS~(ESI,~H)~} \mathrm{m} / \mathrm{z}$ calc'd for $\mathrm{C}_{19} \mathrm{H}_{26} \mathrm{~N}_{3} \mathrm{O}_{3} \mathrm{~S}+(\mathrm{M}+\mathrm{H})^{+} 376.1689$, found 376.1689 .

\section{Transformation of the Product}

Ethanolysis of acylpyrazole: The shlenk flask equipped with a magnetic stirring bar and 3-way glass stopcock was charged with $3 a(32.0 \mathrm{mg}, 0.1 \mathrm{mmol})$. The shlenk flask was evacuated and filled with argon (three cycles). Then dry EtOH $(1.0 \mathrm{~mL}$ ) was added to the shlenk flask. To the solution was added $\mathrm{NaOEt}$ (7.49 $\mathrm{mg}, 0.11 \mathrm{mmol}$ ) at $0{ }^{\circ} \mathrm{C}$ and the mixture was stirred for $14 \mathrm{~h}$ at room temperature. After quenching with $1.0 \mathrm{M} \mathrm{HCl}$, the resultant mixture was extracted with EtOAc. The combined organic layer was washed with brine, and dried over $\mathrm{Na}_{2} \mathrm{SO}_{4}$. After evaporation of the organic solvent under reduced pressure, the crude mixture was purified by silica gel column chromatography to give the $13 a$ ( $27.1 \mathrm{mg}$, $99 \%$ yield)<smiles>CCOC(=O)C([14CH3])[15NH2]</smiles>

ethyl tosylalaninate (13a) (CAS Registry Number 91645-74-8)

$\mathrm{N}$-Boc protection of amino ester ${ }^{5}$ : The shlenk flask equipped with a magnetic stirring bar and 3-way glass stopcock was charged with 13a $(54.3 \mathrm{mg}, 0.2 \mathrm{mmol})$. The shlenk flask was evacuated and filled with argon (three cycles). To a solution of $13 \mathrm{a}$ in $\mathrm{CH}_{2} \mathrm{Cl}_{2}(1.25 \mathrm{ml})$ was added triethylamine $(30.7 \mu \mathrm{l}, 0.22$ $\mathrm{mmol}$ ) and DMAP (4.9 mg, $0.04 \mathrm{mmol})$ followed by $\mathrm{Boc}_{2} \mathrm{O}(55.1 \mu \mathrm{l}, 0.24 \mathrm{mmol})$ at $0{ }^{\circ} \mathrm{C}$ and stirred for $3 \mathrm{~h}$. The mixture was quenched with sat. $\mathrm{NH}_{4} \mathrm{Cl}$ and extracted with EtOAc. After evaporation of the organic solvent under reduced pressure, the crude mixture was purified by silica gel column chromatography to give the $\mathbf{S 1}$ (74.3 $\mathrm{mg}$, quantitative yield) 
<smiles>CCOC(=O)C([14CH3])N([13CH3])C(=O)OCc1ccccc1</smiles>

ethyl N-(tert-butoxycarbonyl)-N-tosylalaninate (S1) (CAS Registry Number 139928-91-9)

Deprotection of Ts group ${ }^{6}$ : The shlenk flask equipped with a magnetic stirring bar and 3-way glass stopcock was charged with $\mathbf{S 1}(37.1 \mathrm{mg}, 0.1 \mathrm{mmol})$. The shlenk flask was evacuated and filled with argon (three cycles). To a solution of $\mathbf{S 1}$ in dry $\mathrm{MeOH}(1.0 \mathrm{ml})$ was added magnesium powder $(12.2 \mathrm{mg}, 0.5$ $\mathrm{mmol}$ ). The mixture was sonicated for $1 \mathrm{~h}$ (reaction temperature should be kept around $30^{\circ} \mathrm{C}$ during the reaction). After quenching with $0.5 \mathrm{M} \mathrm{HCl}$, the resultant mixture was extracted with EtOAc. The combined organic layer was washed with brine and dried over $\mathrm{Na}_{2} \mathrm{SO}_{4}$. After evaporation of the organic solvent under reduced pressure, the crude mixture was purified by silica gel column chromatography to give the 11a (20.1 $\mathrm{mg}, 99 \%$ yield).<smiles>COC(=O)C(C)NC(C)(C)C</smiles>

methyl (tert-butoxycarbonyl)alaninate (11a): (CAS Registry Number 72604-32-1)

Hydrolysis of acylpyrazole: To a solution of $3 a(32.0 \mathrm{mg}, 0.1 \mathrm{mmol})$ in $\mathrm{H}_{2} \mathrm{O} / \mathrm{MeOH}(1.0 \mathrm{ml} / 0.2 \mathrm{ml})$ was added $4.0 \mathrm{M} \mathrm{NaOH}$ aq. (37.5 $\mu \mathrm{l}, 0.15 \mathrm{mmol})$. After stirring for $2 \mathrm{~h}$ at room temperature, $1.0 \mathrm{M} \mathrm{HCl}$ was added. The resultant mixture was extracted with EtOAc and combined organic layer was extracted with sat. $\mathrm{NaHCO}_{3}$ Then the combined aqueous layer was acidified with $1.0 \mathrm{M} \mathrm{HCl}$. The acidified mixture was extracted with EtOAc and the combined organic layer was concentrated under reduced pressure to give the pure carboxylic acid $12 \mathrm{a}(23.6 \mathrm{mg}, 97 \%$ yield) 
<smiles>CC(N)C(=O)O</smiles>

tosylalanine (12a)(CAS Registry Number 4816-81-3)

Direct amidation with H-Gly-OMe: To a suspension of $3 a(31.1 \mathrm{mg}, 0.1 \mathrm{mmol})$ and $\mathrm{H}-\mathrm{Gly}-\mathrm{OMe} \cdot \mathrm{HCl}(25.1$ $\mathrm{mg}, 0.2 \mathrm{mmol})$ in toluene $(0.4 \mathrm{ml})$ was added triethylamine $(28.0 \mu \mathrm{l}, 0.2 \mathrm{mmol})$ at room temperature. After $1 \mathrm{~h}$ stirring at $50{ }^{\circ} \mathrm{C}$, it was quenched by $1.0 \mathrm{~N} \mathrm{HCl}$. The resultant mixture was extracted with $\mathrm{CH}_{2} \mathrm{Cl}_{2}$, and combined organic layers were washed with sat. $\mathrm{NaHCO}_{3}$ solution and brined successively. The resulting organic layers were dried over $\mathrm{Na}_{2} \mathrm{SO}_{4}$. After removal of solvent under reduced pressure, the crude mixture was purified by silica gel column chromatography (67\%-83\% EtOAc/n-hexane) to afford desired product 14a (31.6 mg, quantitative yield).<smiles></smiles>

methyl tosylalanylglycinate (14a) (CAS Registry Number 875762-72-4)

Ketone formation with Grignard reagent ${ }^{7}$ : The shlenk flask equipped with a magnetic stirring bar and 3way glass stopcock was charged with $3 a(32.0 \mathrm{mg}, 0.1 \mathrm{mmol})$. The shlenk flask was evacuated and filled with argon (three cycles). Then dry $\mathrm{Et}_{2} \mathrm{O}(1.0 \mathrm{~mL})$ was added to the shlenk flask and the reaction mixture was cooled to $0{ }^{\circ} \mathrm{C}$. To the solution was added $1.0 \mathrm{M} \mathrm{PhMgBr}$ in THF solution $(0.2 \mathrm{ml}, 0.2 \mathrm{mmol})$ at $0{ }^{\circ} \mathrm{C}$ and the reaction mixture was stirred for $4 \mathrm{~h}$ at room temperature. After quenching with $1.0 \mathrm{M} \mathrm{HCl}$, the resultant mixture was extracted with EtOAc. The combined organic layer was washed with brine, and dried over $\mathrm{Na}_{2} \mathrm{SO}_{4}$. After evaporation of the organic solvent under reduced pressure, the crude mixture was purified by silica gel column chromatography to give the product $15 a$ ( $28.4 \mathrm{mg}, 94 \%$ yield) 
<smiles>CC(N)C(=O)c1ccccc1</smiles>

4-methyl-N-(1-oxo-1-phenylpropan-2-yl)benzenesulfonamide (15a) (CAS Registry Number 64274-26-6)

Selective reduction to aldehyde ${ }^{8}$ : The well-dried shlenk flask equipped with a magnetic stirring bar and 3-way glass stopcock was charged with $\mathrm{LiAlH}_{4}(5.54 \mathrm{mg}, 0.15 \mathrm{mmol})$ and dry THF $(1.22 \mathrm{~mL})$ at $-40{ }^{\circ} \mathrm{C}$. To the solution was added $81.0 \mathrm{mM}$ 3a solution in dry THF $(1.23 \mathrm{ml}, 0.1 \mathrm{mmol})$ was added. After TLC analysis showed the starting material was consumed $(30 \mathrm{~min}), 1.0 \mathrm{M} \mathrm{HCl}$ was added at $-40{ }^{\circ} \mathrm{C}$ and stirred for $15 \mathrm{~min}$. The reaction mixture was directly filtered through celite and washed with EtOAc. After evaporation of the organic solvent under reduced pressure, the crude mixture was purified by silica gel column chromatography to give the desired product $16 a(18.9 \mathrm{mg}, 83 \%$ yield)<smiles></smiles>

4-methyl-N-(1-oxopropan-2-yl)benzenesulfonamide (16a) (CAS Registry Number 57483-26-8)

Reduction to alcohol ${ }^{9}$ : To a solution of $3 a(32.0 \mathrm{mg}, 0.1 \mathrm{mmol})$ in $\mathrm{THF} / \mathrm{H}_{2} \mathrm{O}(1.54 \mathrm{ml} / 0.39 \mathrm{ml})$ was added $\mathrm{NaBH}_{4}(30.3 \mathrm{mg}, 0.8 \mathrm{mmol})$ at $0{ }^{\circ} \mathrm{C}$ and the mixture was stirred for $6 \mathrm{~h}$ at room temperature. After quenching with $1.0 \mathrm{M} \mathrm{HCl}$, the resultant mixture was extracted with EtOAc. The combined organic layer was washed with brine and dried over $\mathrm{Na}_{2} \mathrm{SO}_{4}$. After evaporation of the organic solvent under reduced pressure, the crude mixture was purified by silica gel column chromatography to give the 17 a $(23.0 \mathrm{mg}$, quantitative yield).<smiles>CC(CO)C[AsH3-]</smiles>

N-(1-hydroxypropan-2-yl)-4-methylbenzenesulfonamide (17a) (CAS Registry Number 114663-10-4) 


\section{References}

1. Yamada, Y.; Yamamoto, T.; Okawara, M. Chem. Lett. 1975, 361.

2. Kashima, C.; Harada, H.; Kita, I.; Fukuchi, I.; Hosomi, A. Synthesis, 1994, 61.

3. Smirnov, V. O.; Khomutova, Y. A.; Tartakovsky, V. A.; loffe, S. L. Eur. J. Org. Chem. 2012, 3377.

4. For the preparation of corresponding carboxylic acid: DeMartino, M. P.; Chen, K.; Baran, P. S. J. Am. Chem. Soc. 2008, 130, 11546

5. Aponick, A.; Li, C.-Y.; Malinge, J.; Marques, E. F. Org. Lett. 2009, 11, 4624.

6. (a) Juhl, K.; Gathergood, N.; Jørgensen, K. A. Angew. Chem. Int. Ed. 2001, 40, 2995. (b) Nyasse, B.; Grehn, L.; Ragnarsson, U. Chem. Commun. 1997,1017

7. Kashima, C.; Kita, I.; Takahashi, K.; Hosomi, A. J. Heterocyclic Chem. 1995, 32, 25.

8. Remuzon, P.; Bouzard, D.; Guiol, C.; Jacquet, J. P. J. Med. Chem. 1992, 35, 2898.

9. Ishihara, K.; Fushimi, M. J. Am. Chem. Soc. 2008, 130, 7532. 


\section{NMR Spectra of New Compounds}

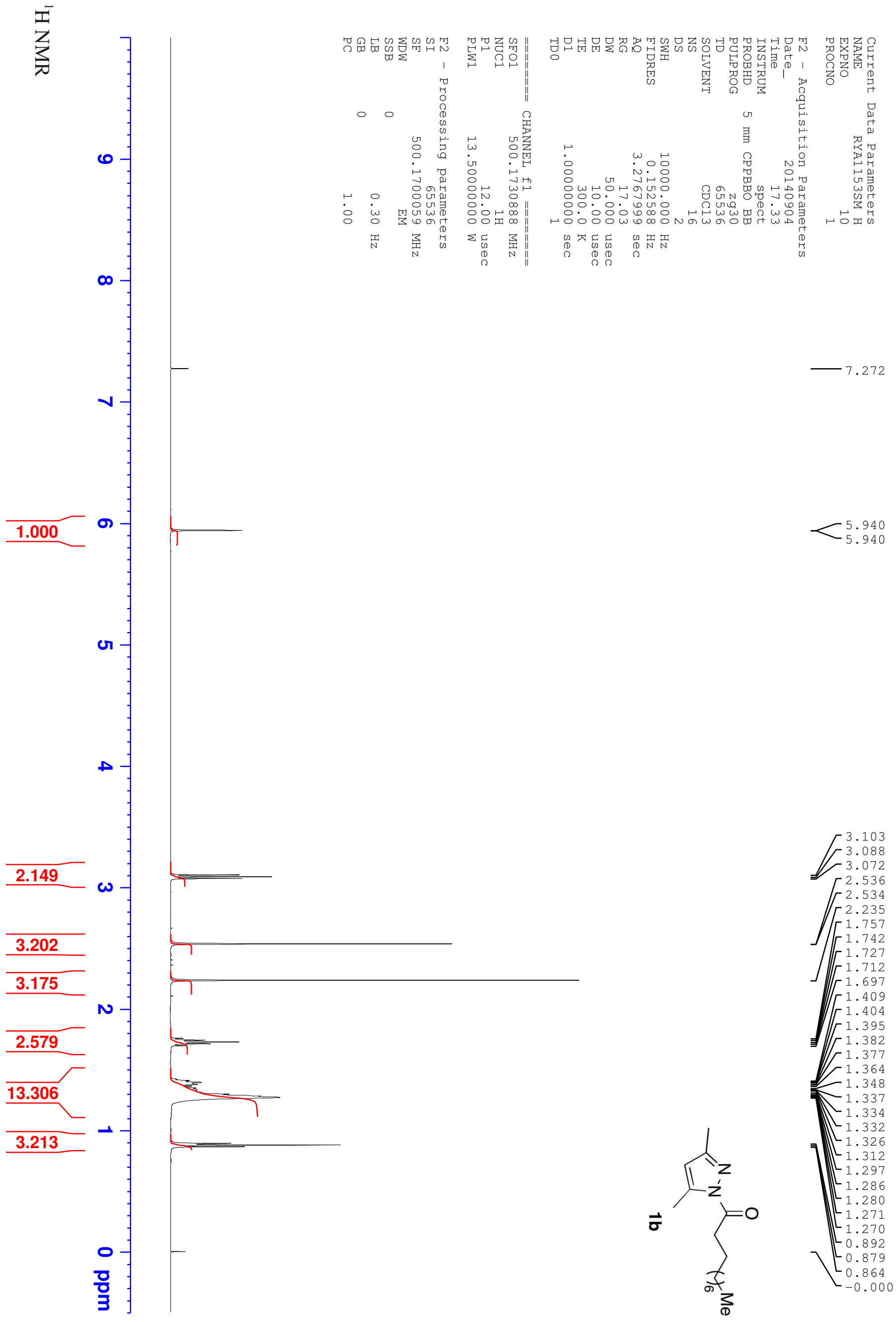


$\overbrace{0}^{\bar{\omega}}$

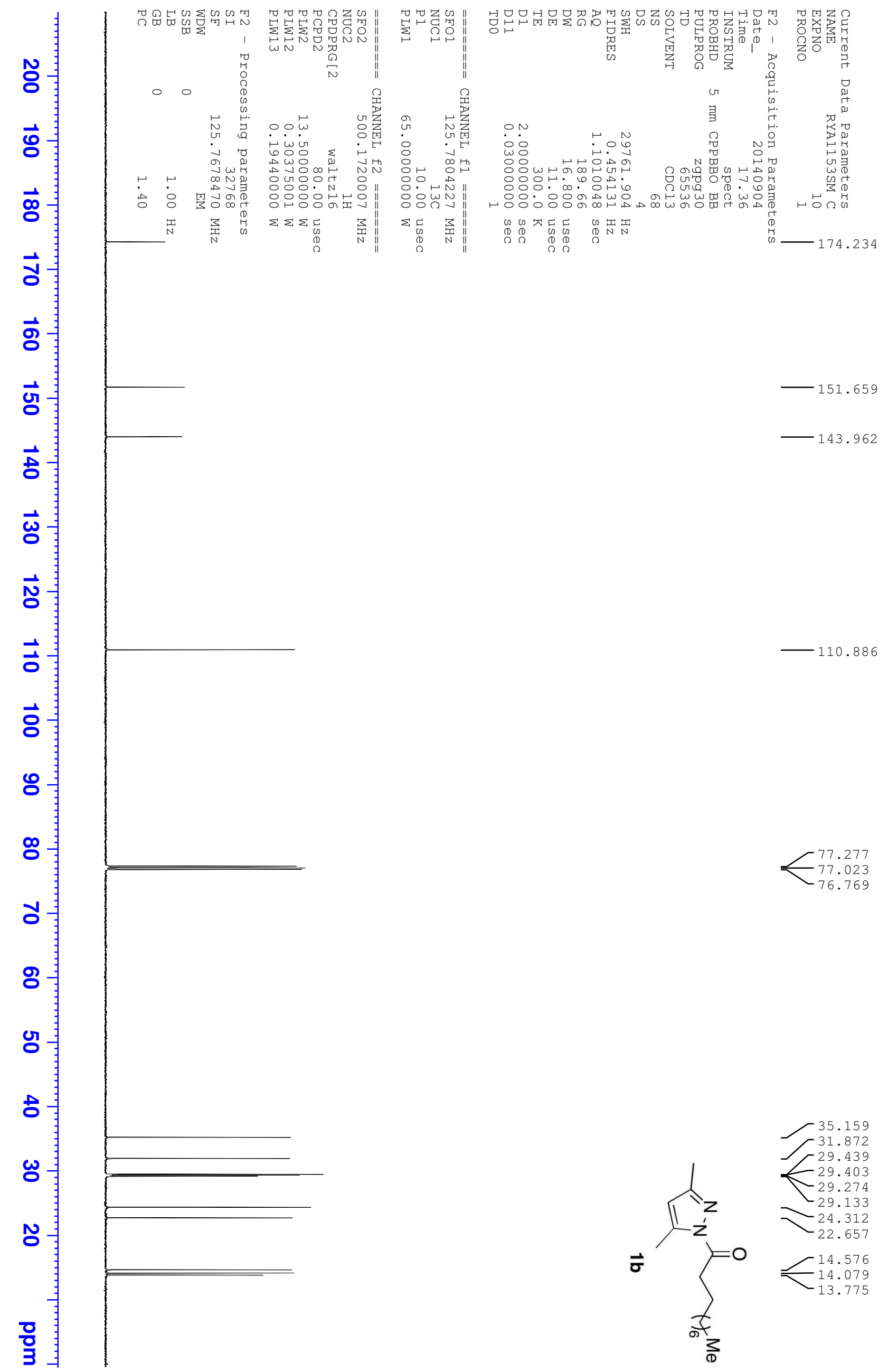




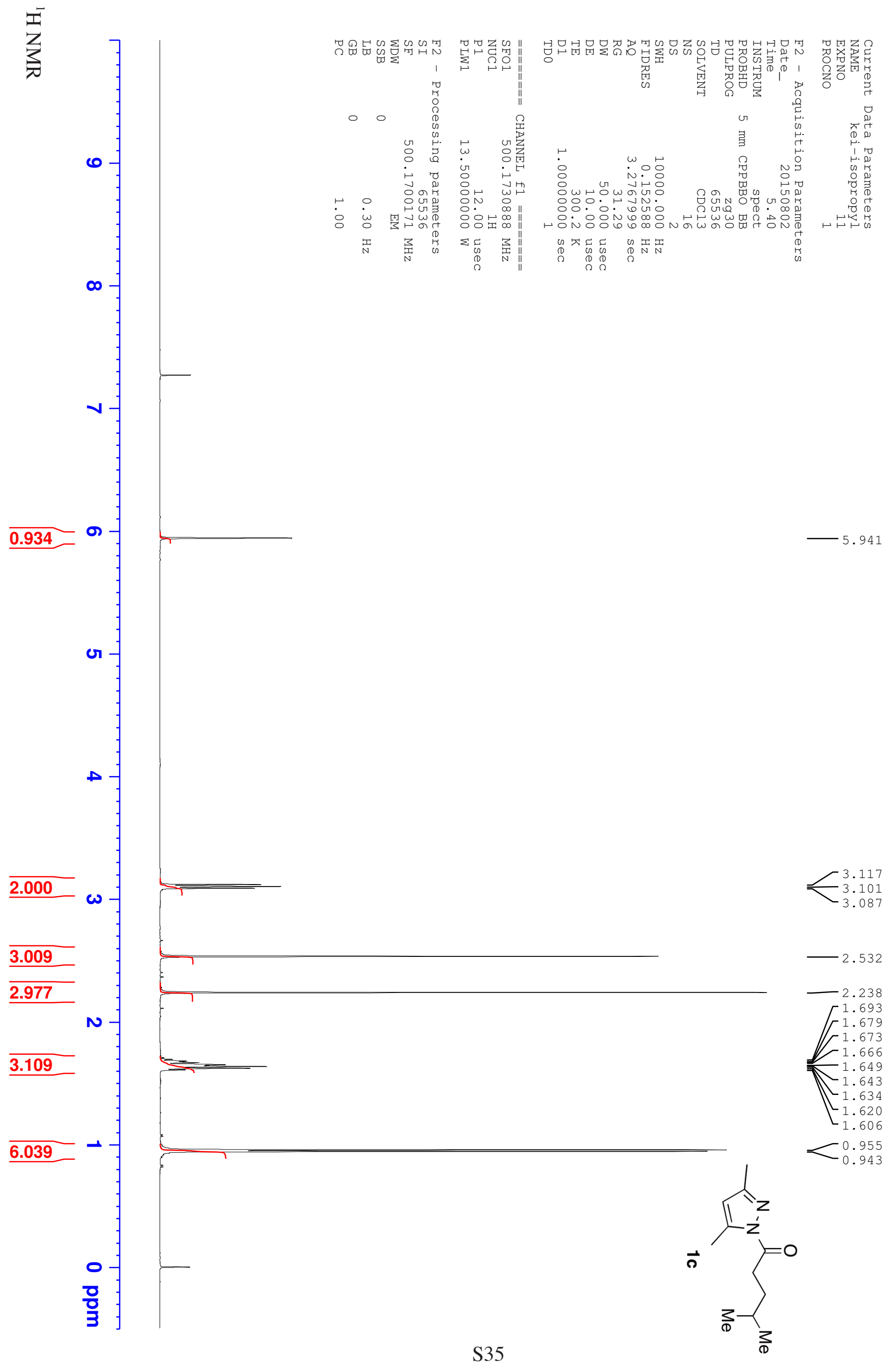


$\overbrace{0}^{\bar{\omega}}$

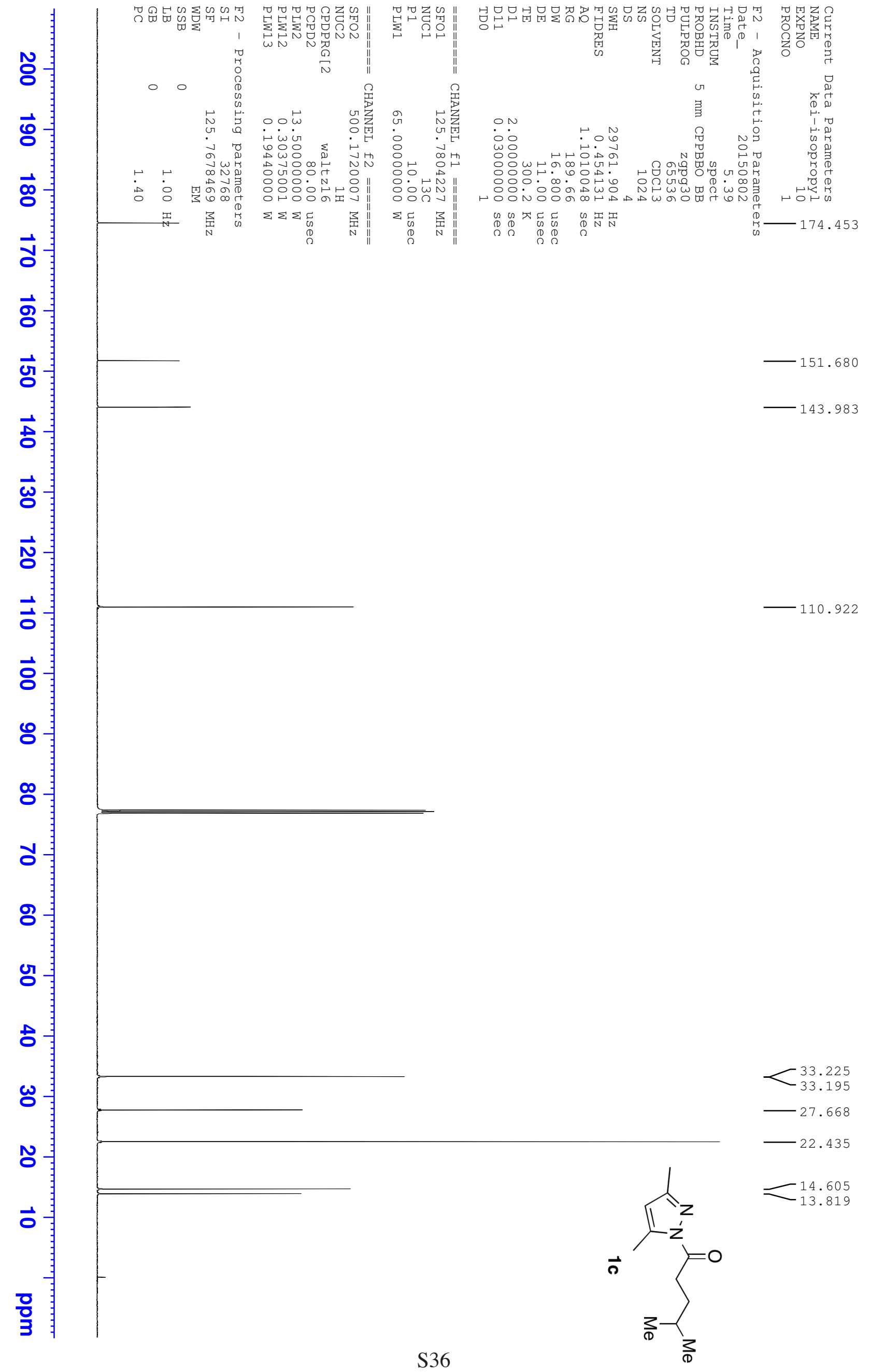




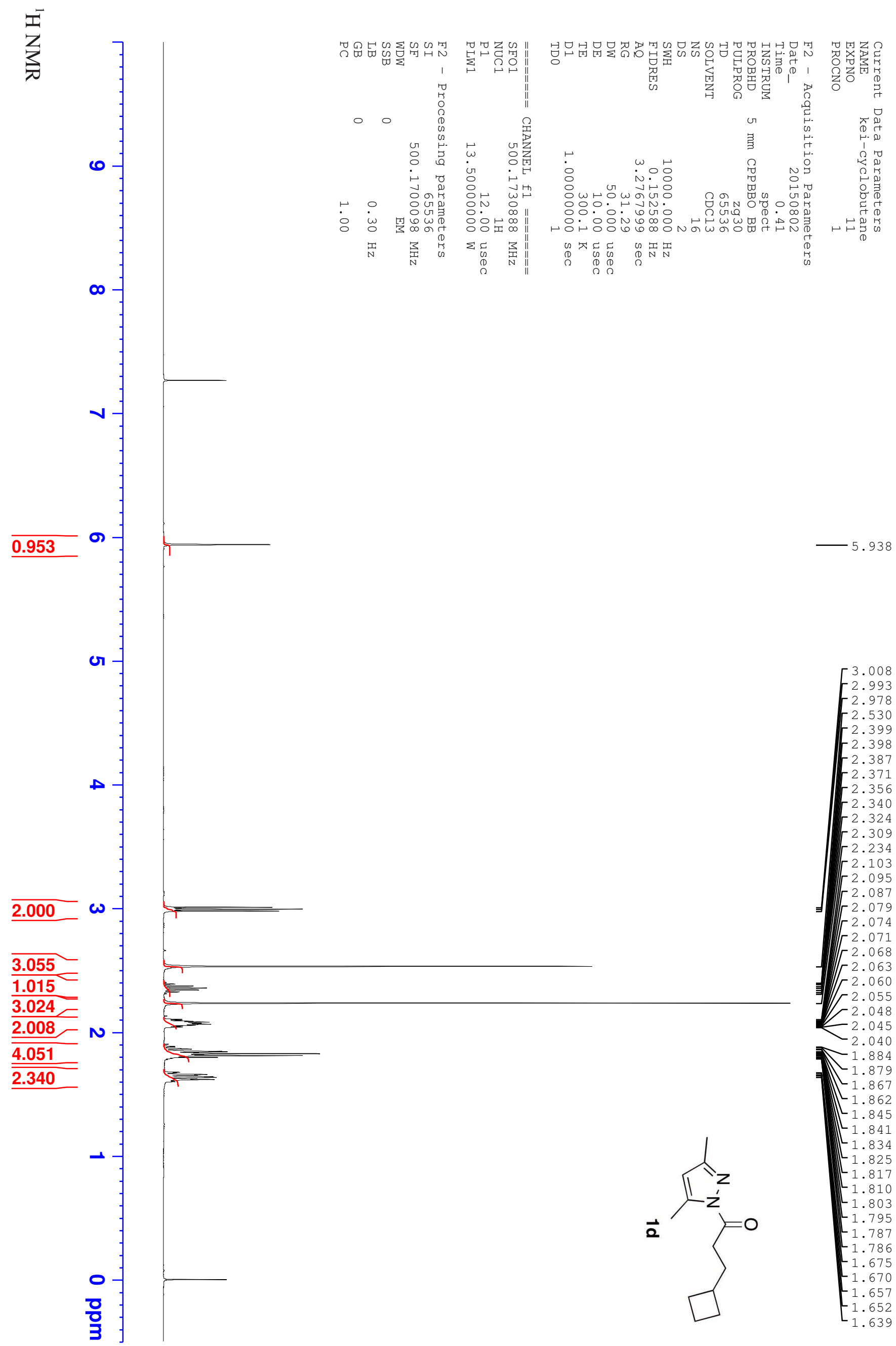


$\overbrace{}^{2}$

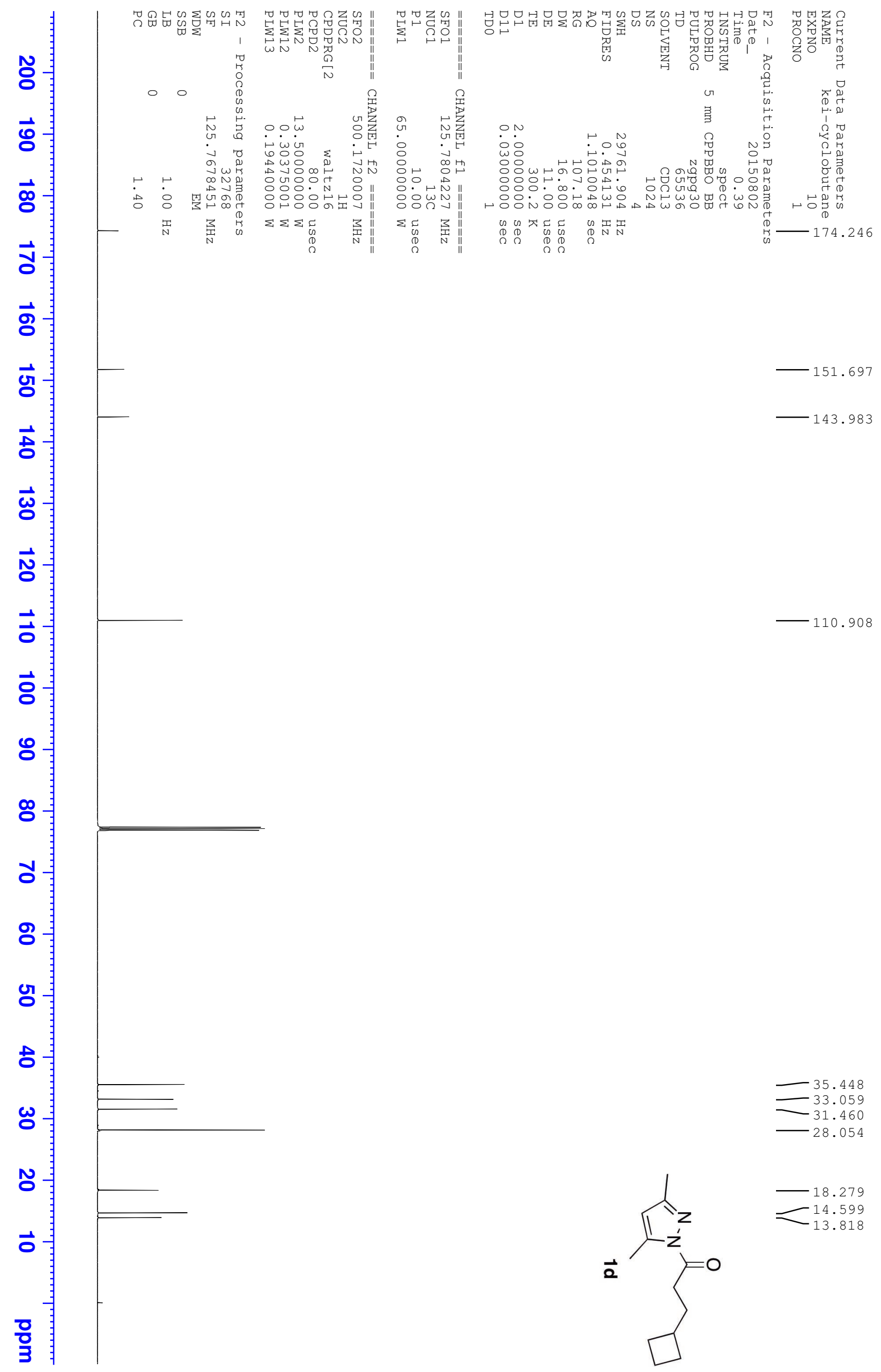




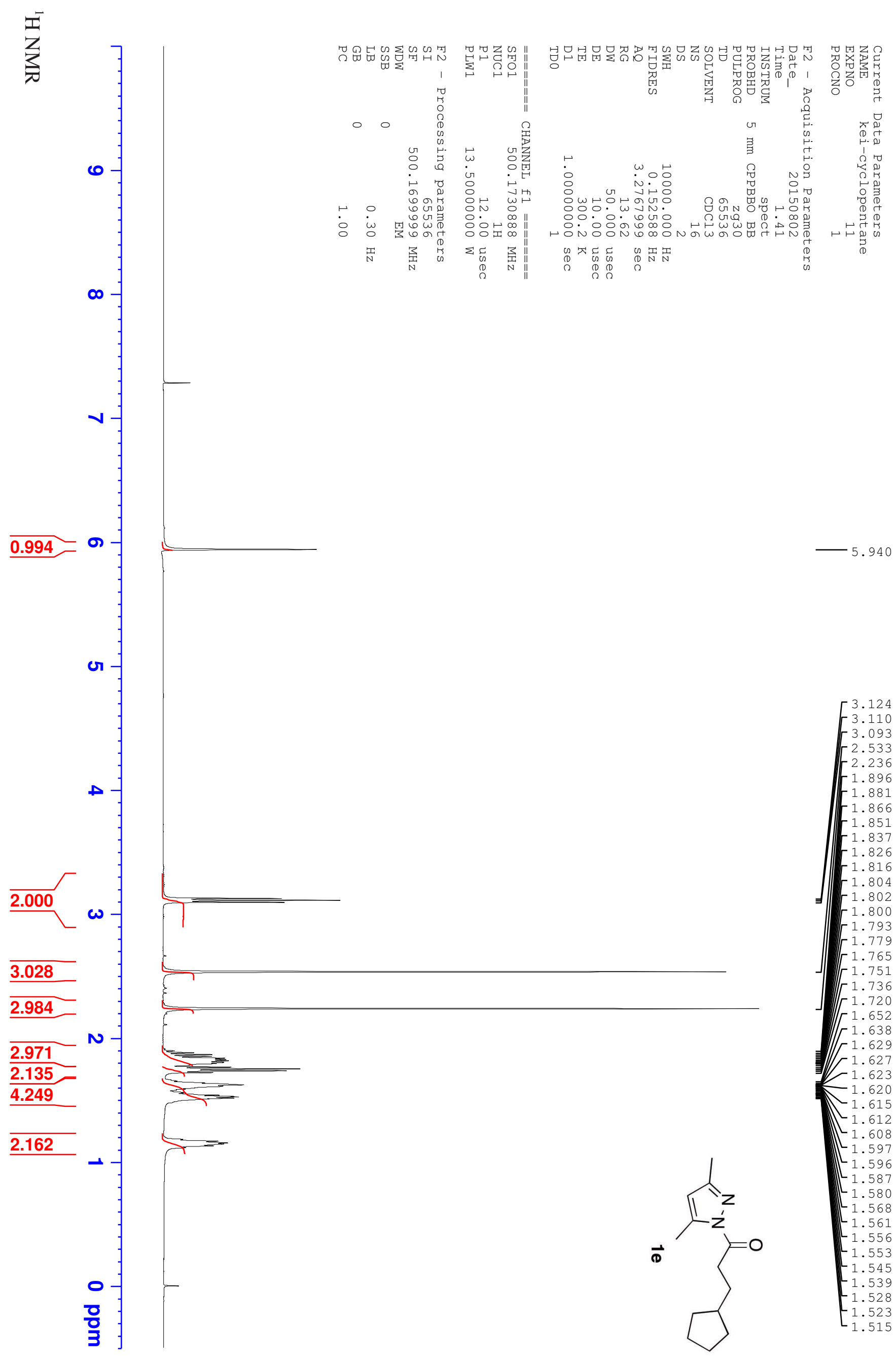


$\overbrace{}^{3}$

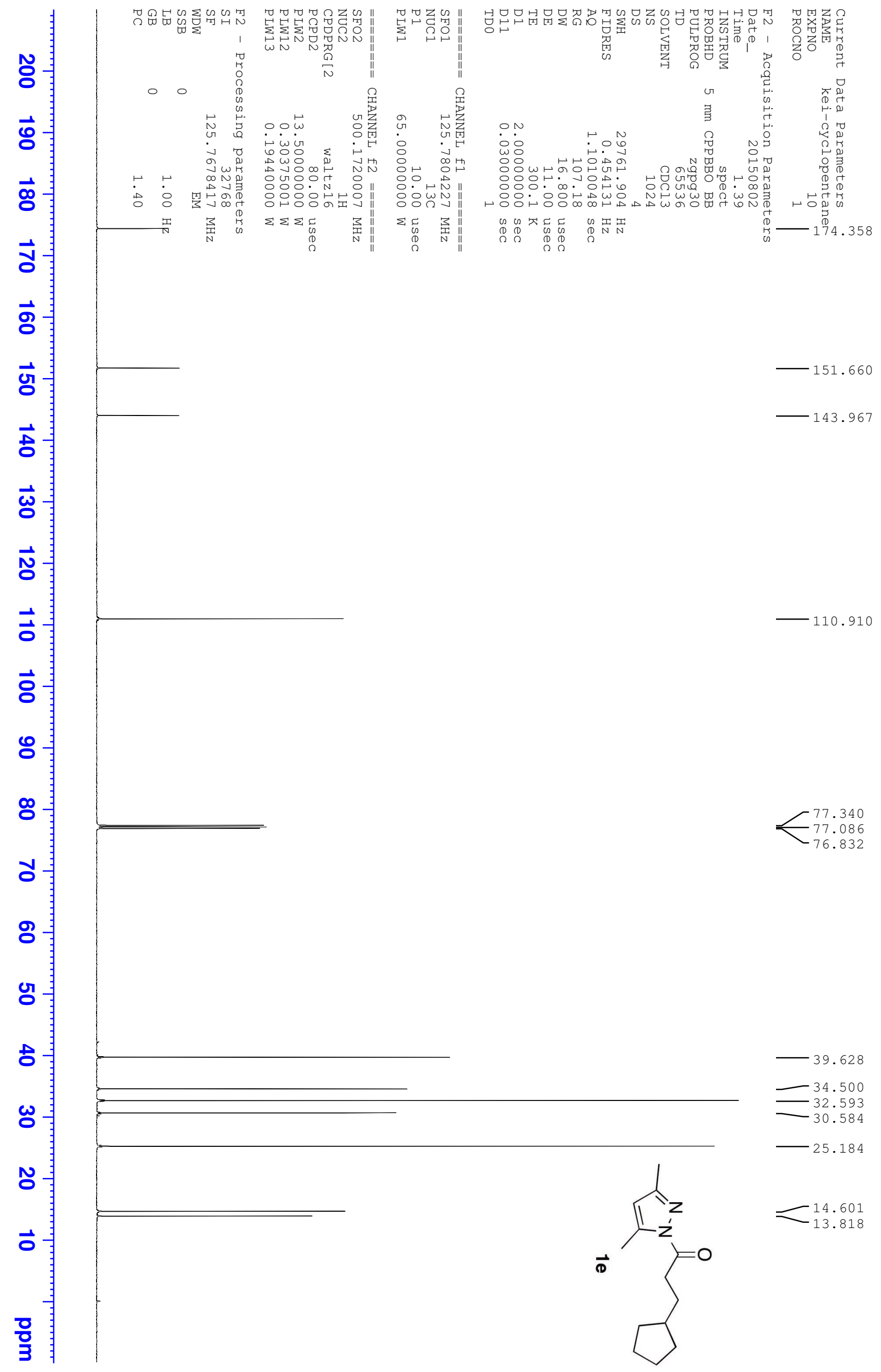




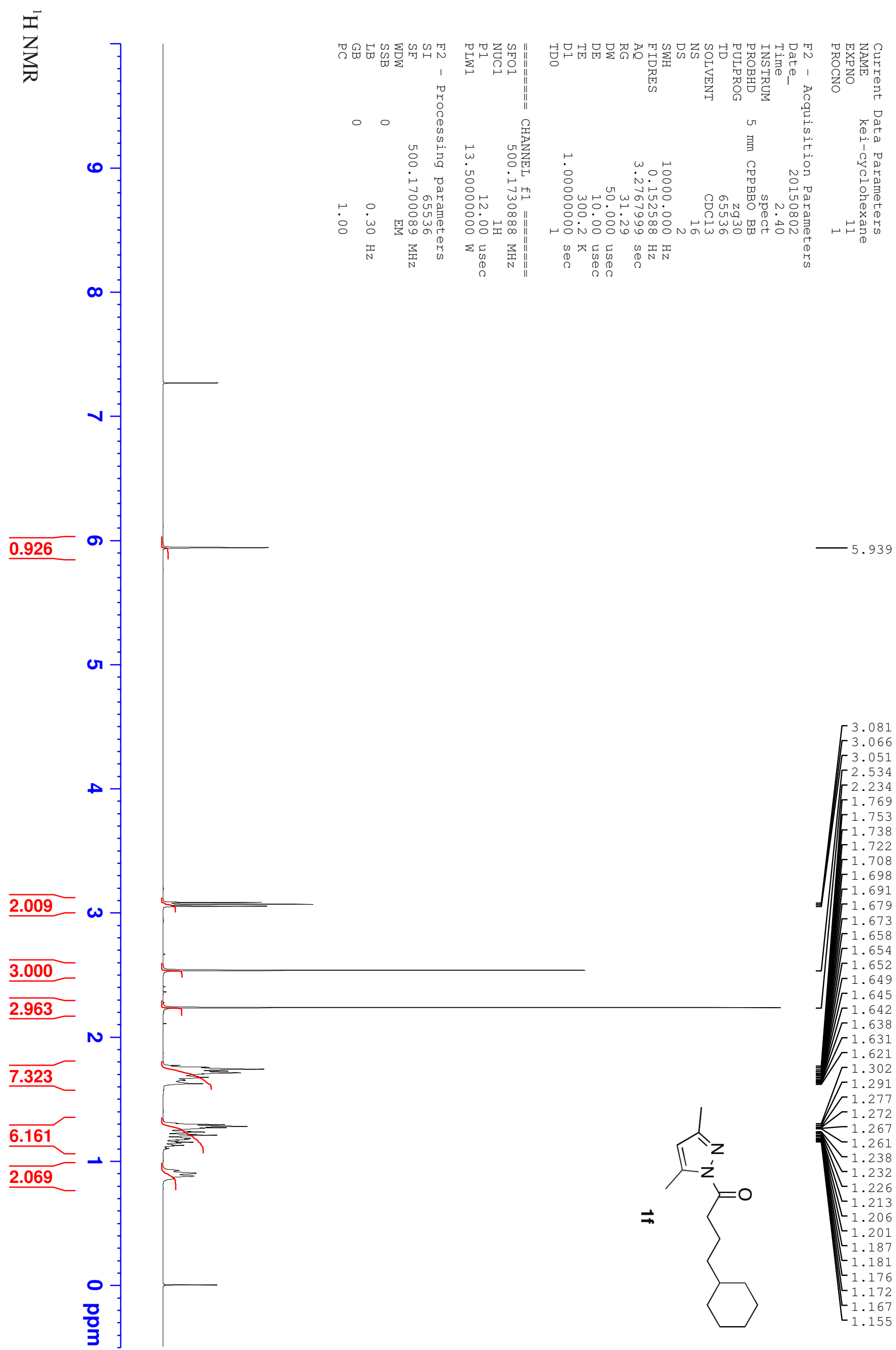


$\overbrace{}^{3}$

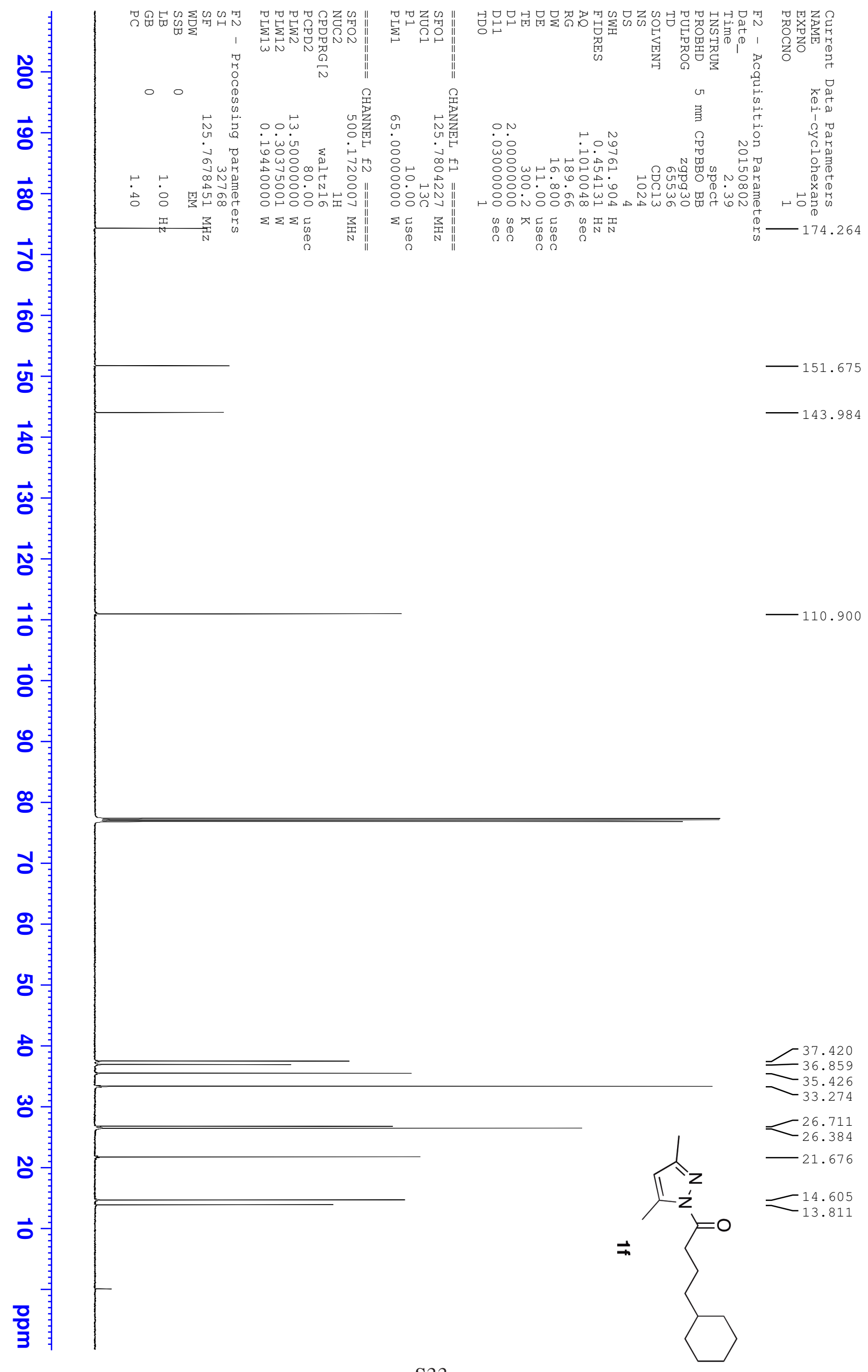




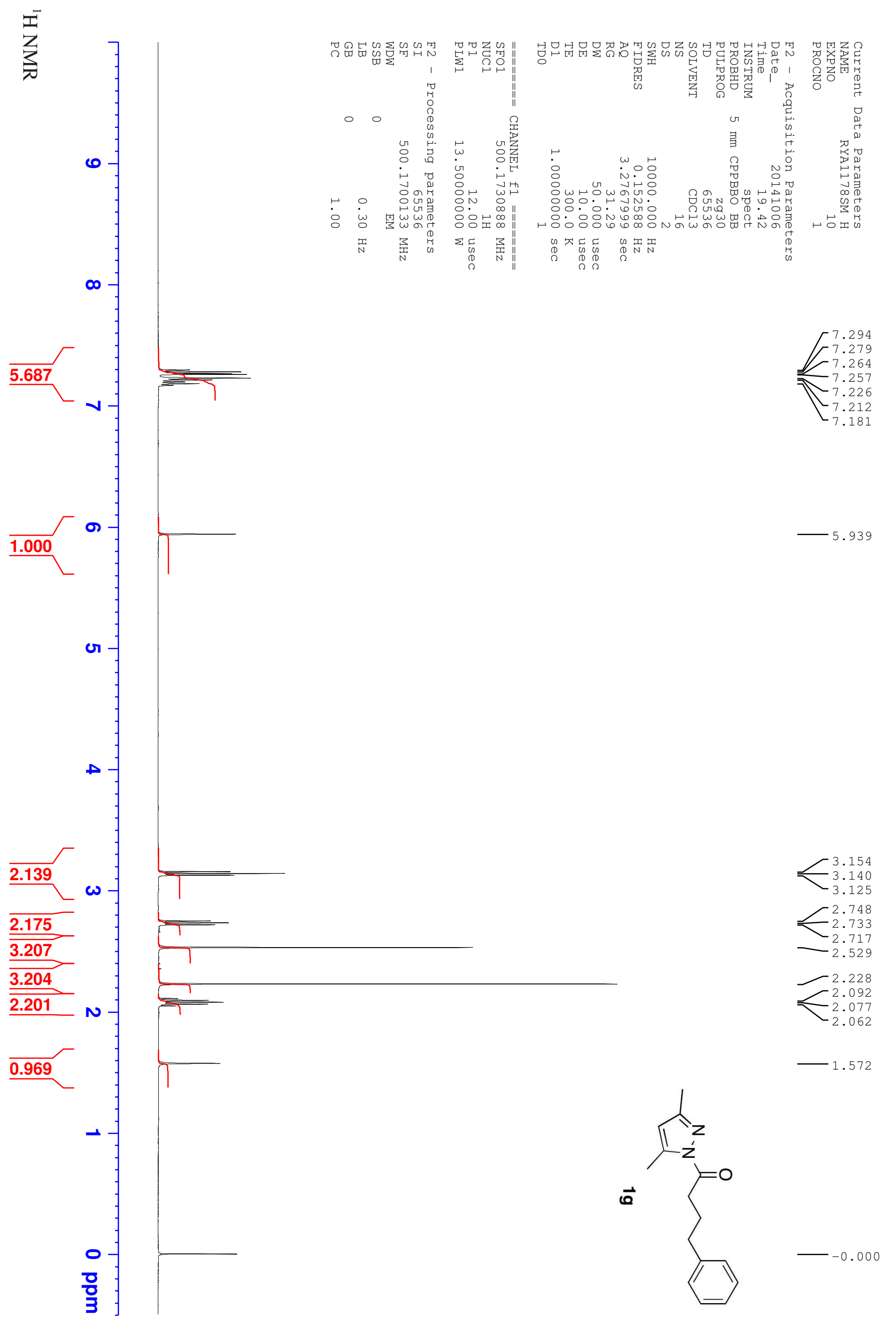


$\overbrace{}^{2}$
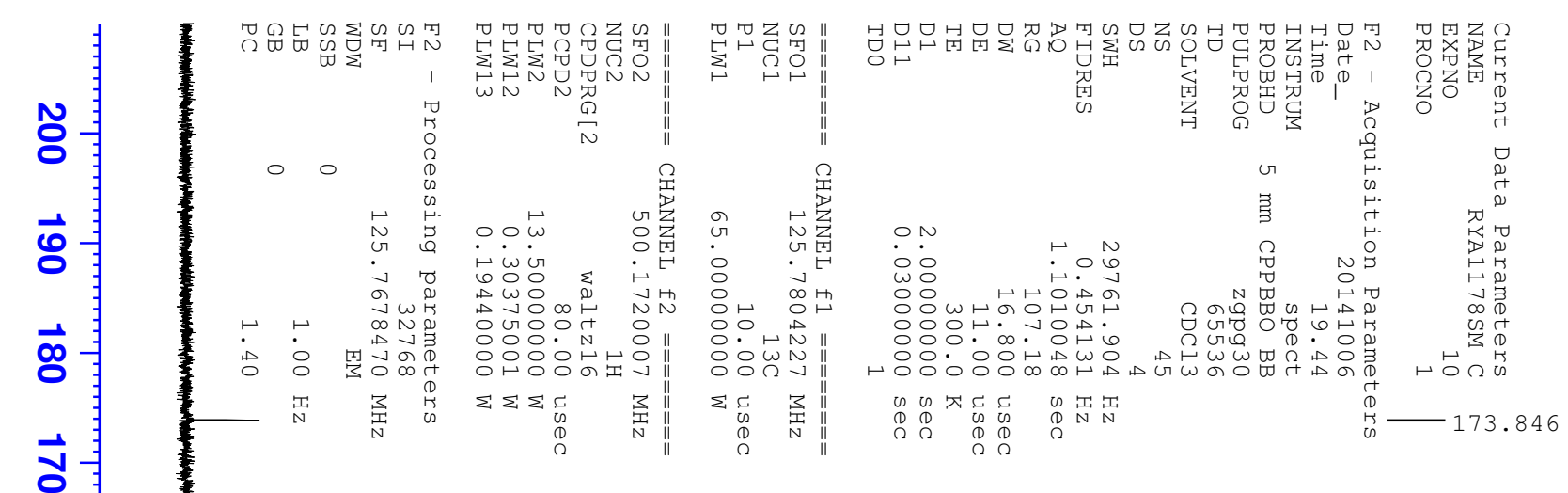

$\vec{\circ}$

ज

$\vec{f}$

$-143.993$

141.686

$\vec{\omega}$

$\vec{N}$

$\vec{a}$

$\overrightarrow{\mathrm{s}}$

8

ø

○

8

용

to

๗

ข

-

$\overrightarrow{0}$

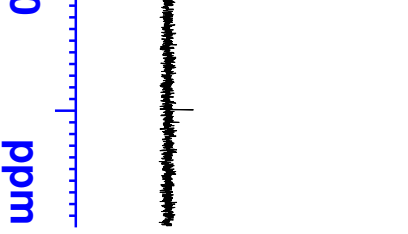

$-110.981$

77.274

77.019
-76.765
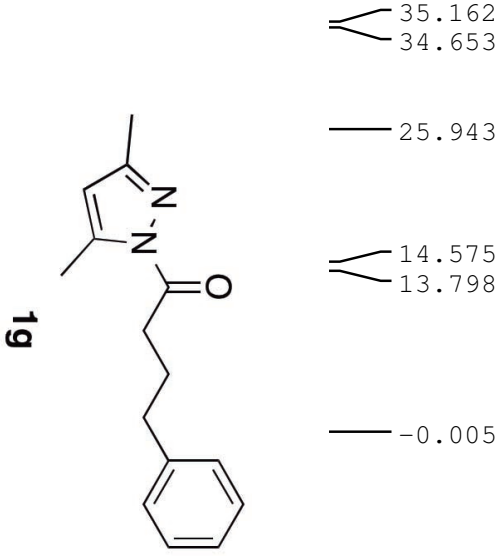

$-14.575$

13.798 


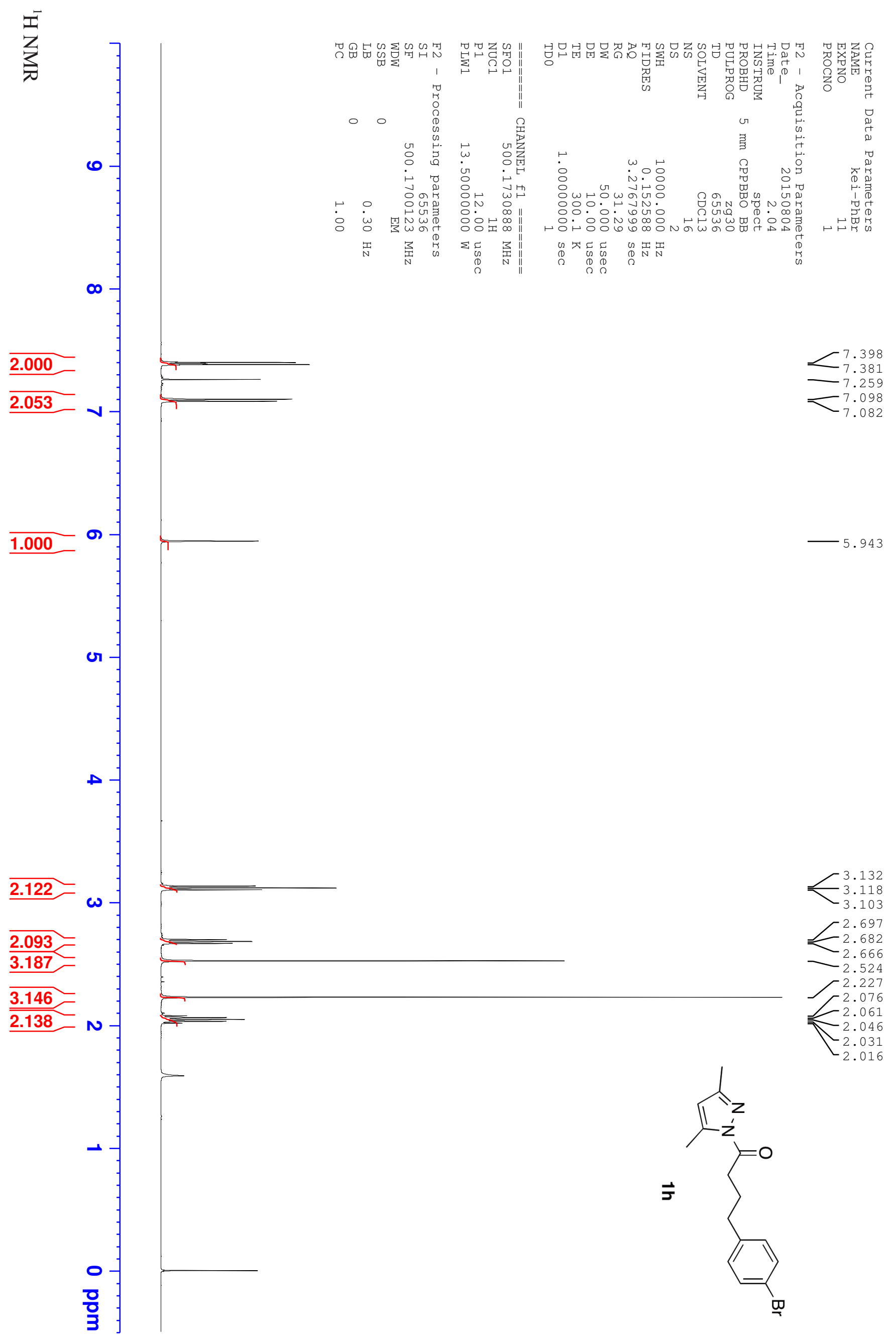


$\overbrace{}^{2}$

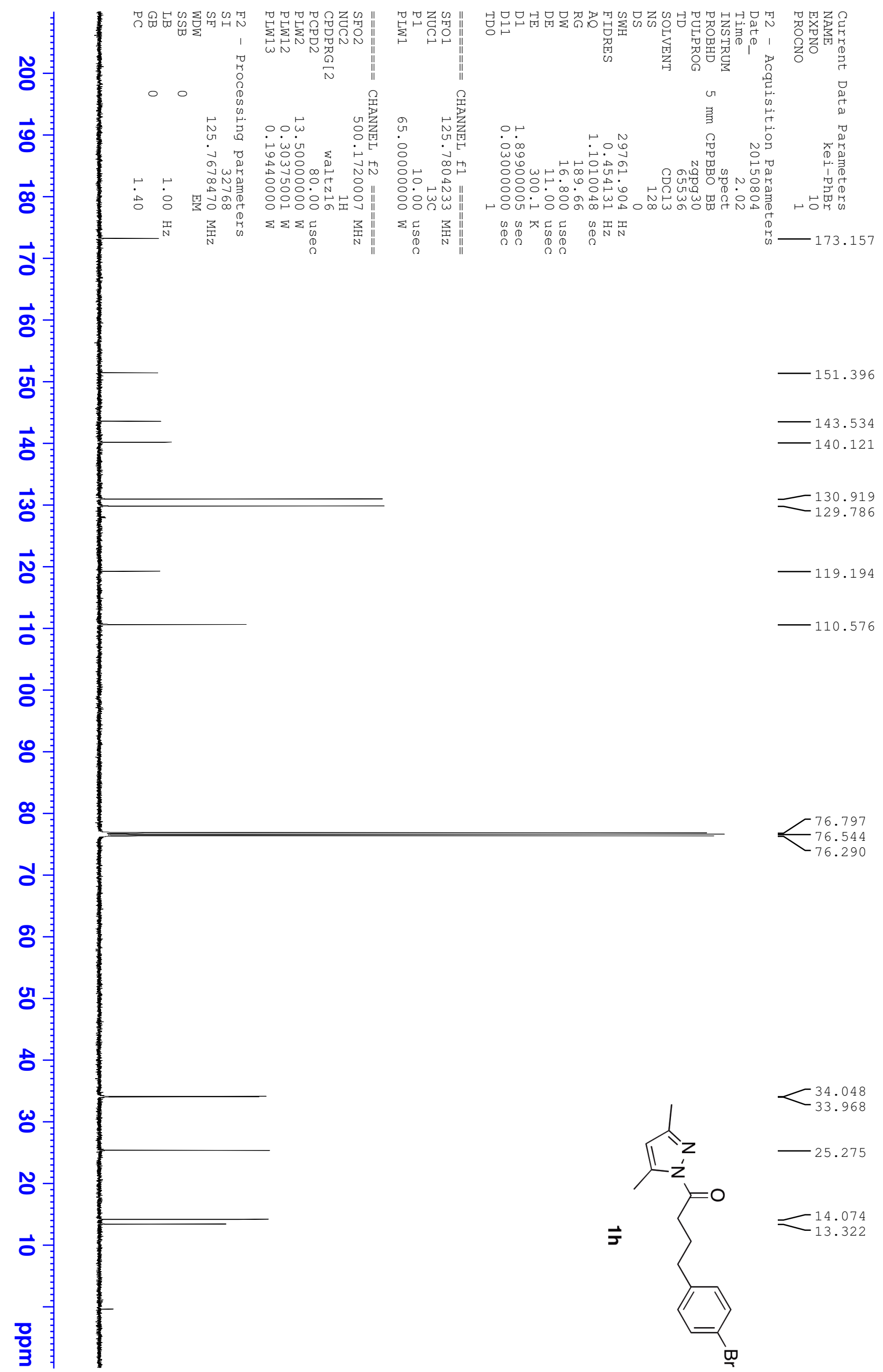




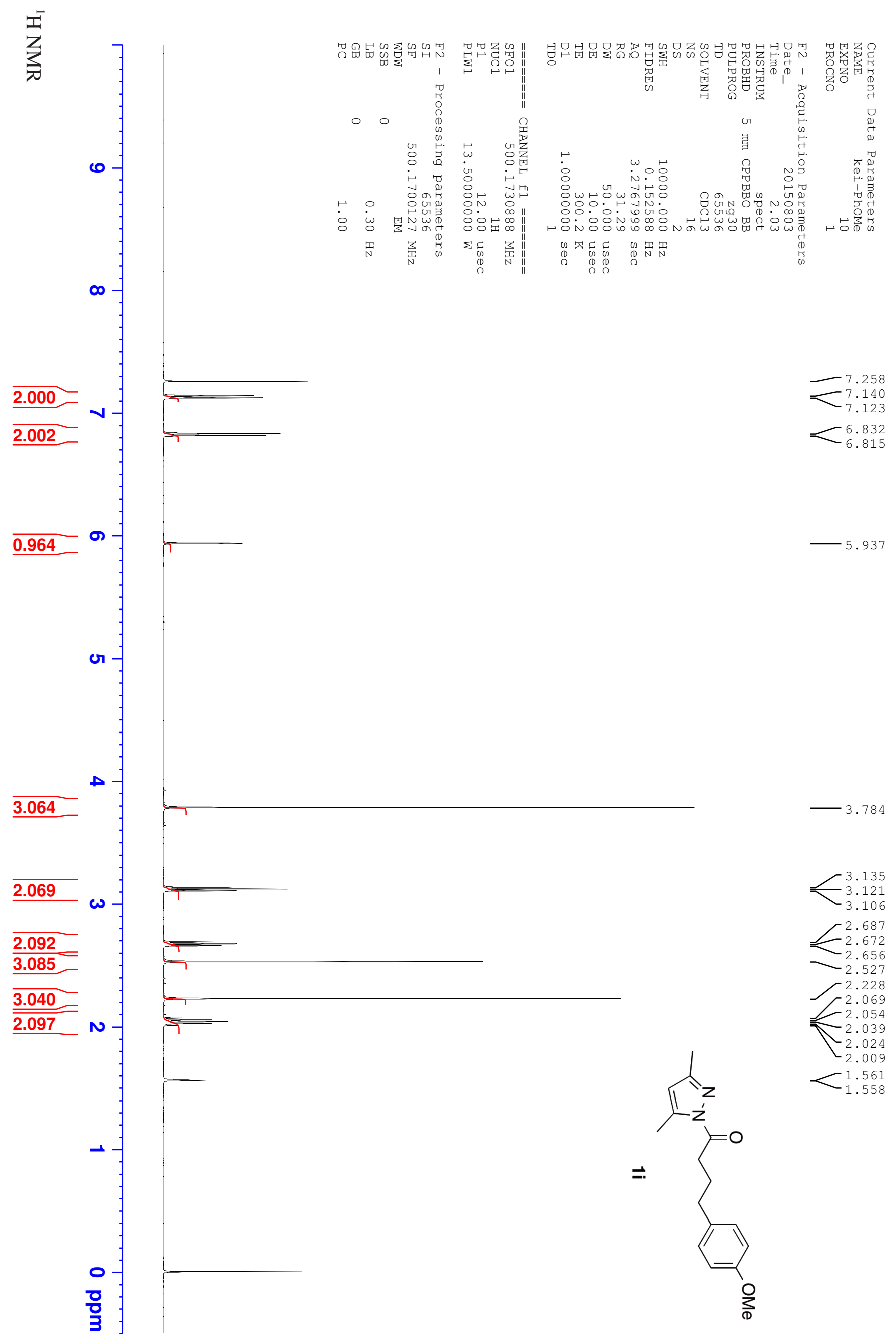


$\overbrace{0}^{\bar{\omega}}$

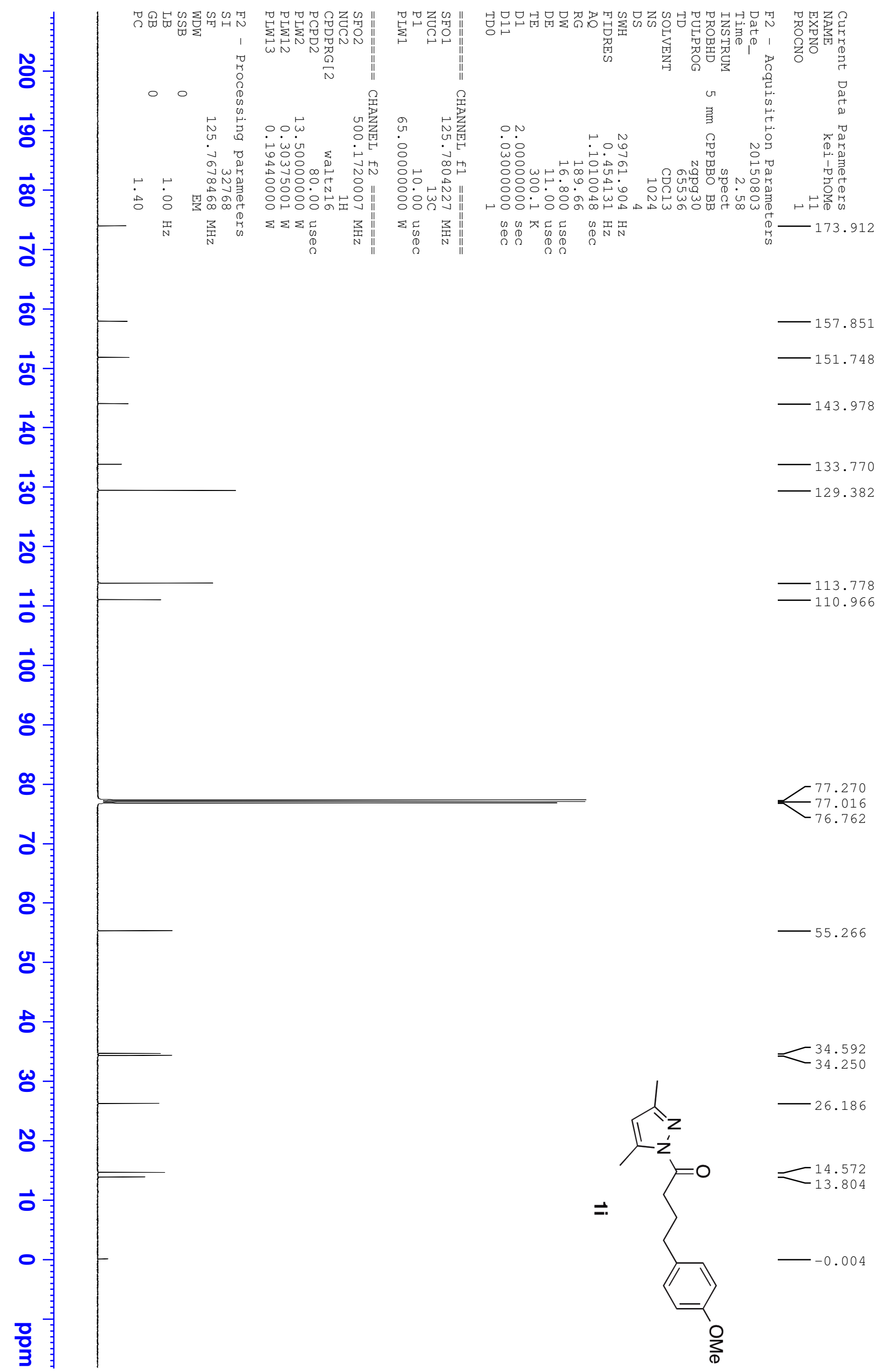




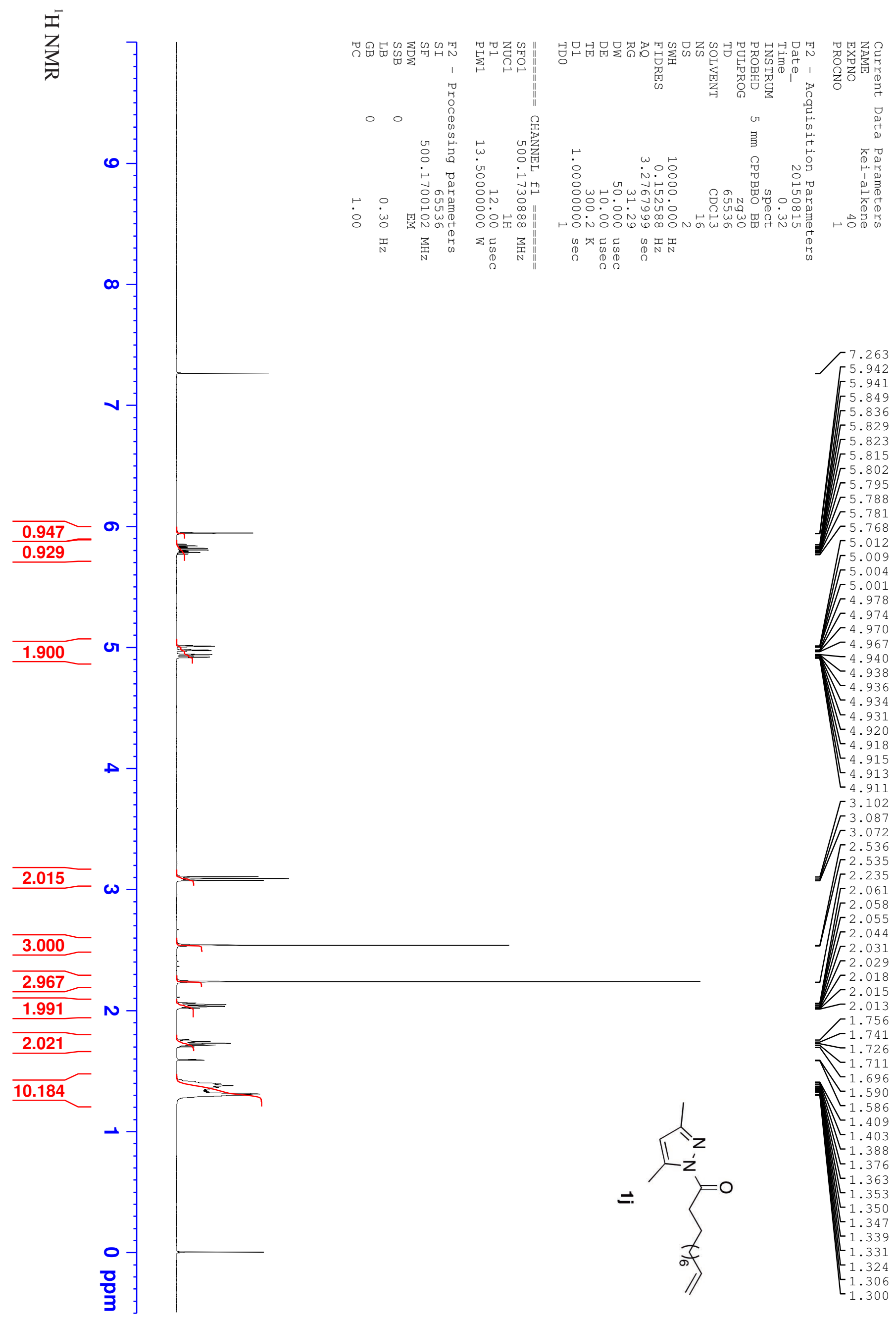


$\overbrace{}^{2}$

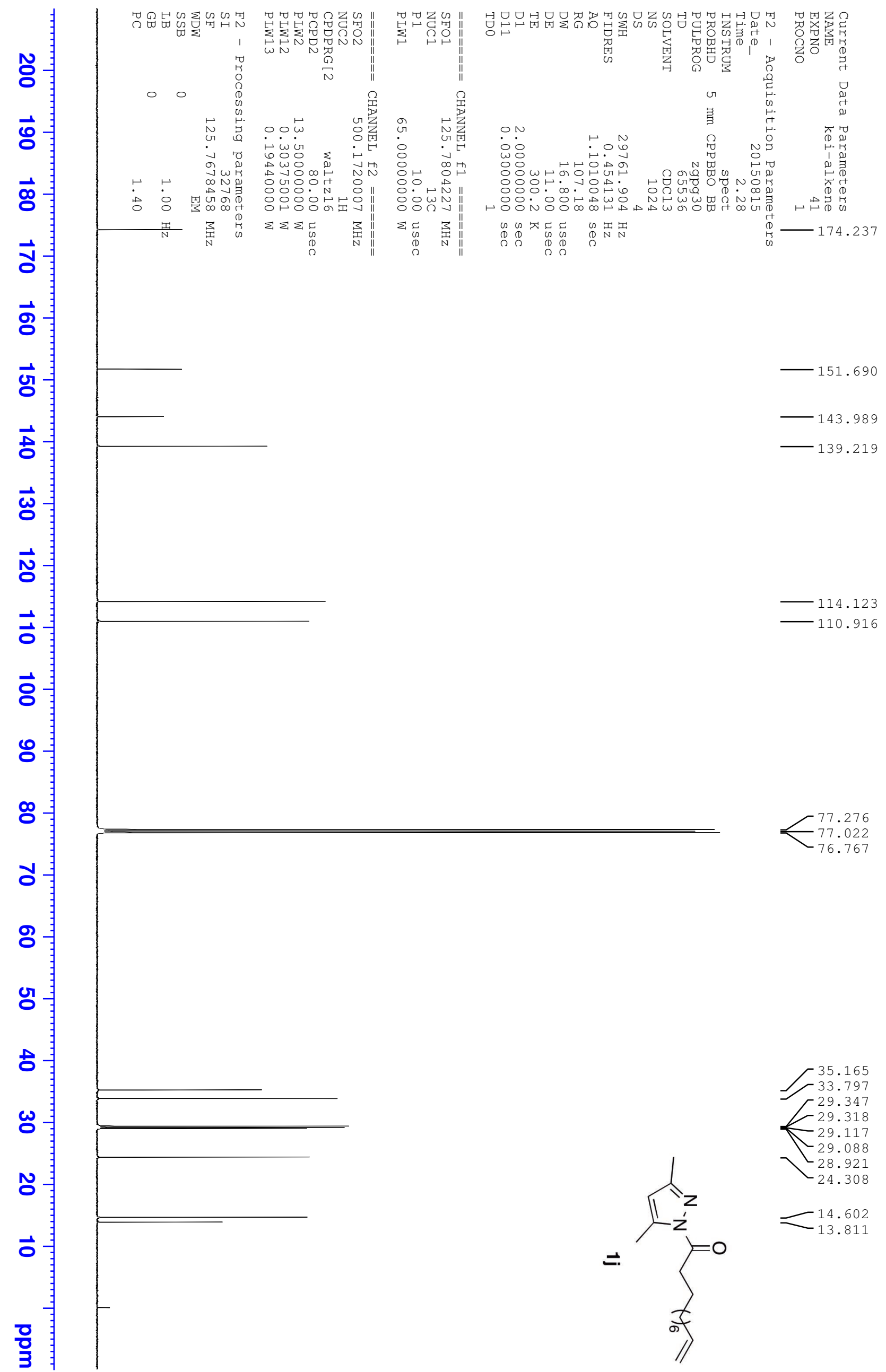




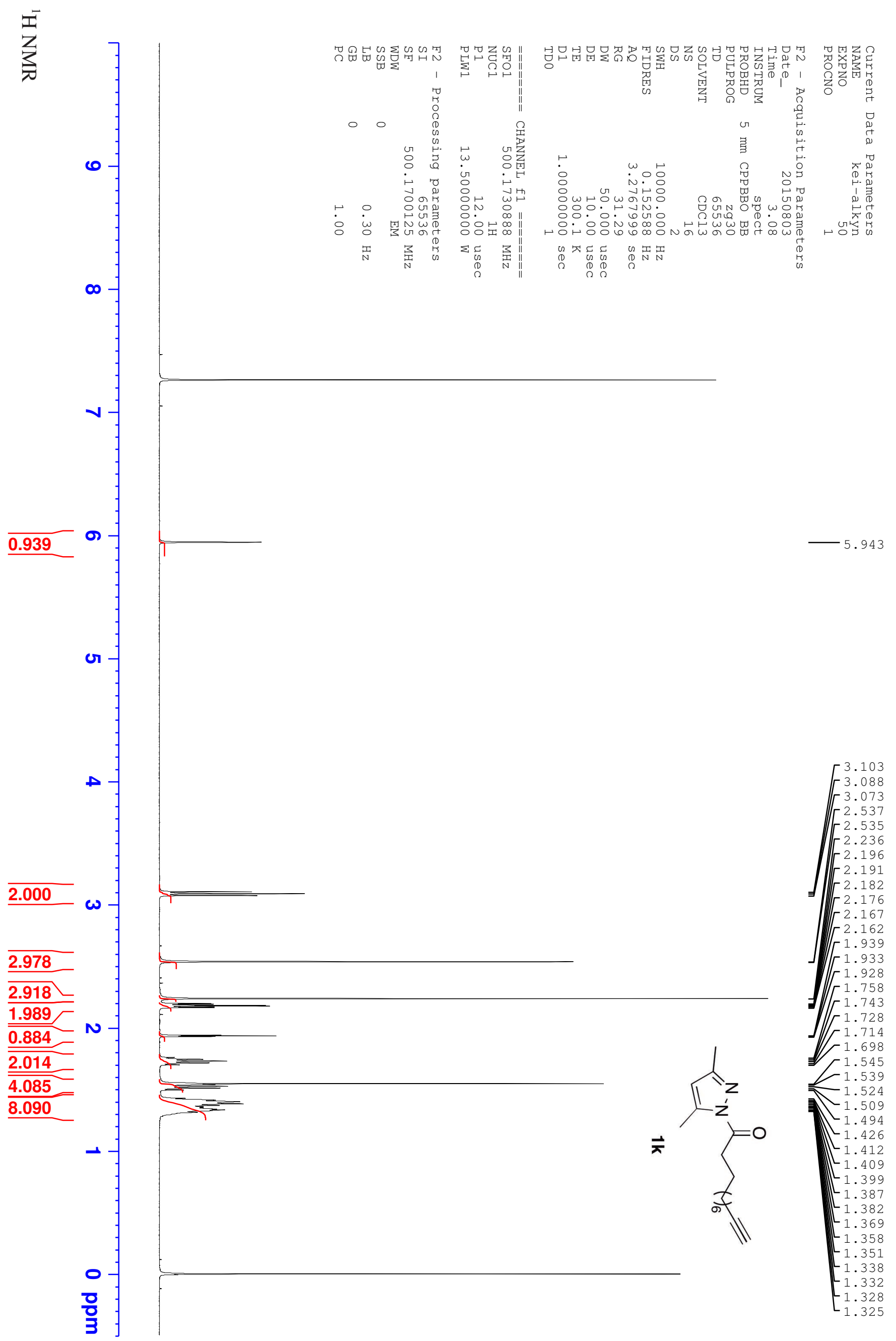


$\overbrace{}^{2}$

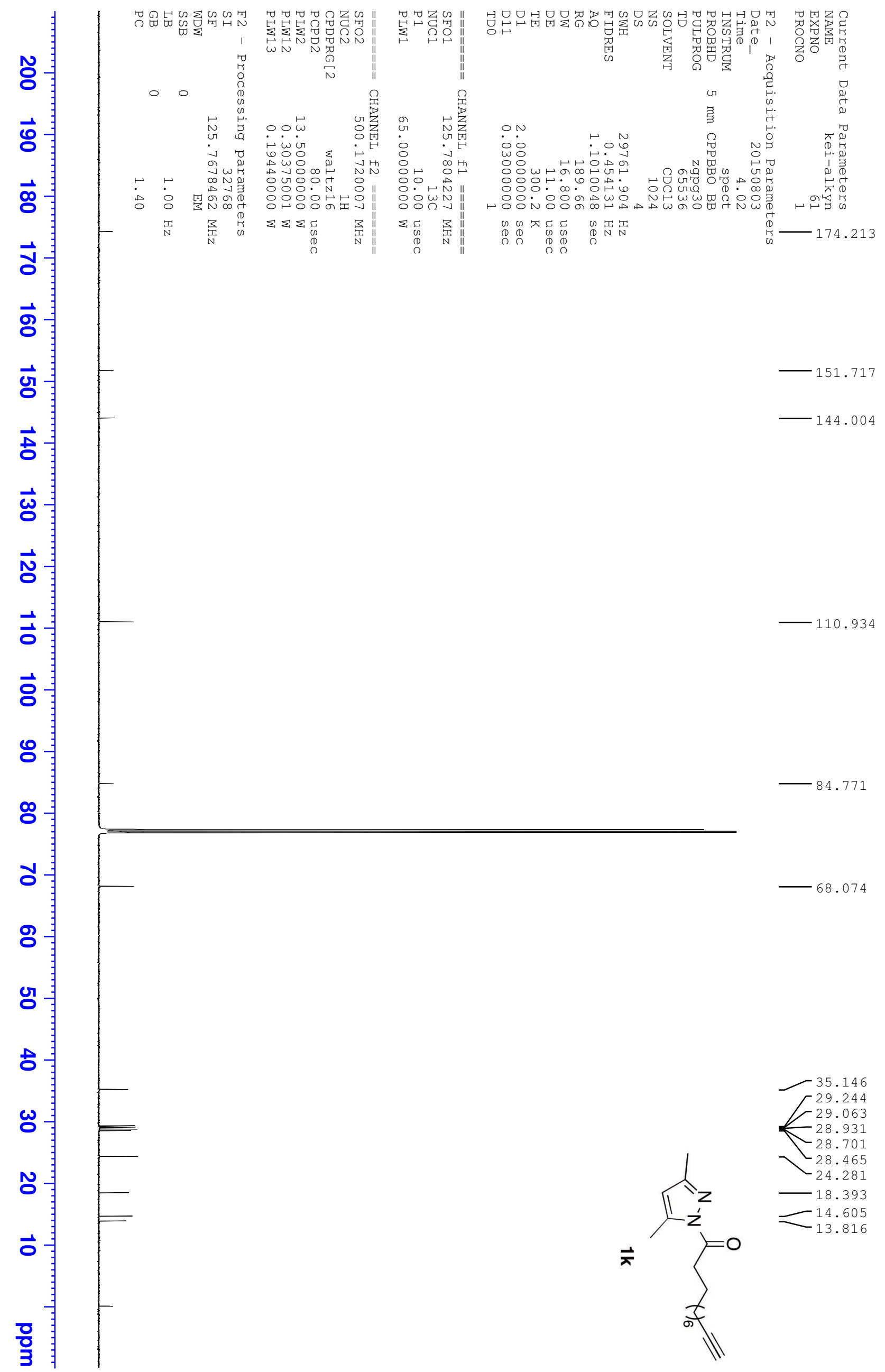




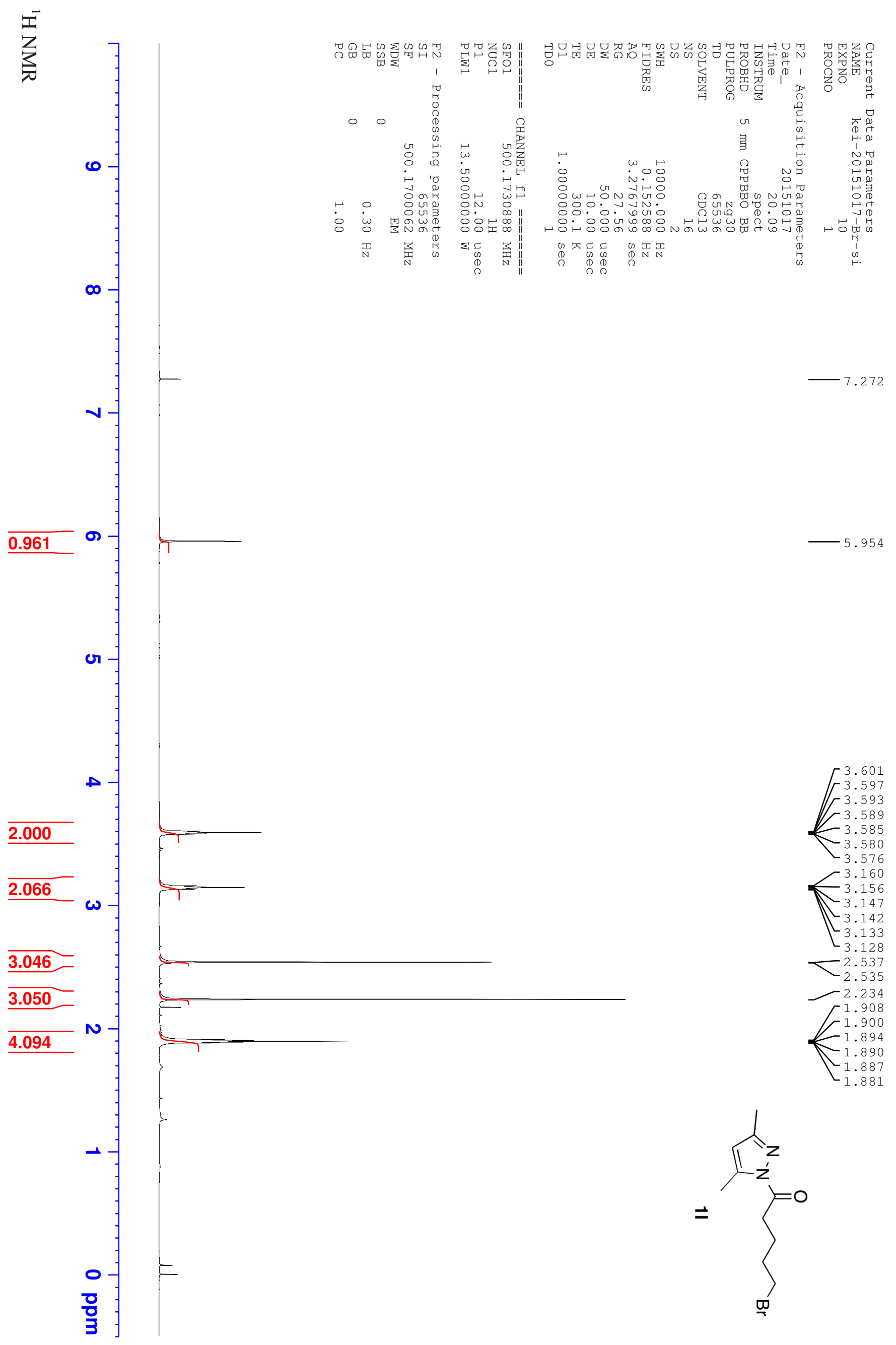


$\overbrace{}^{\bar{\omega}}$

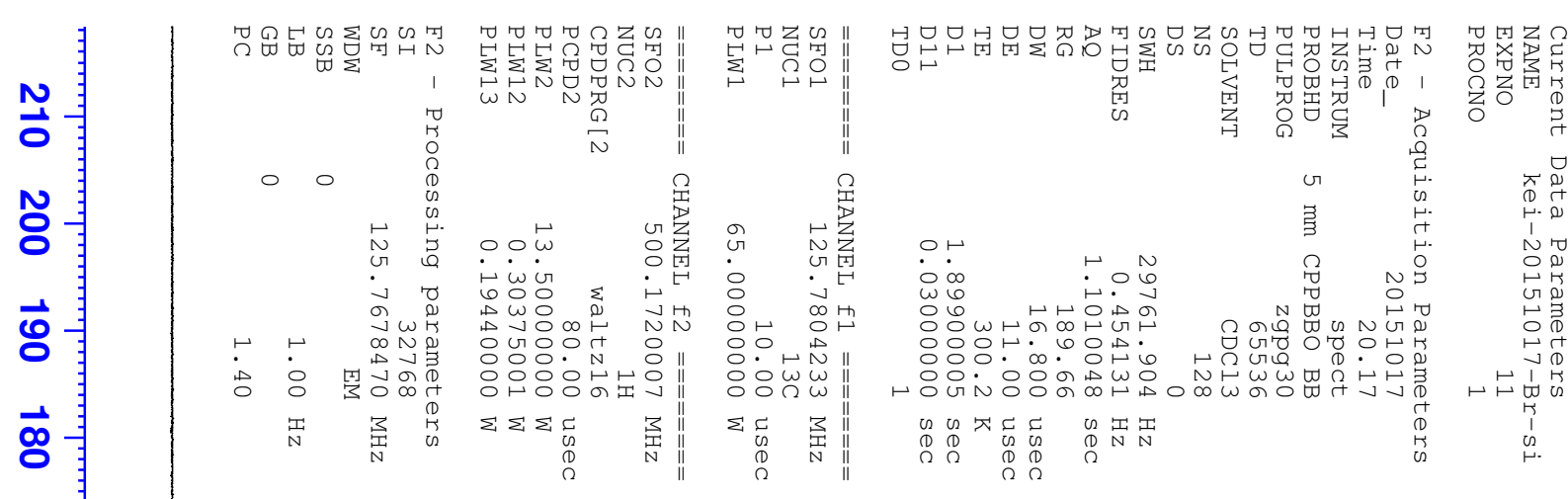

$\overrightarrow{0}$

$\overrightarrow{8}$

ज्ञ

룽

$\overrightarrow{\text { ஸे }}$

$\vec{N}$

$\overrightarrow{0}$

ฉ

$\mathscr{8}$

ळ

J

g

잉

\&

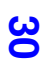

$\circ$

은

o

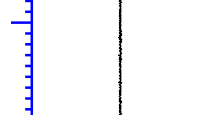

青永

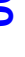

8

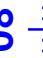

8

굥- 


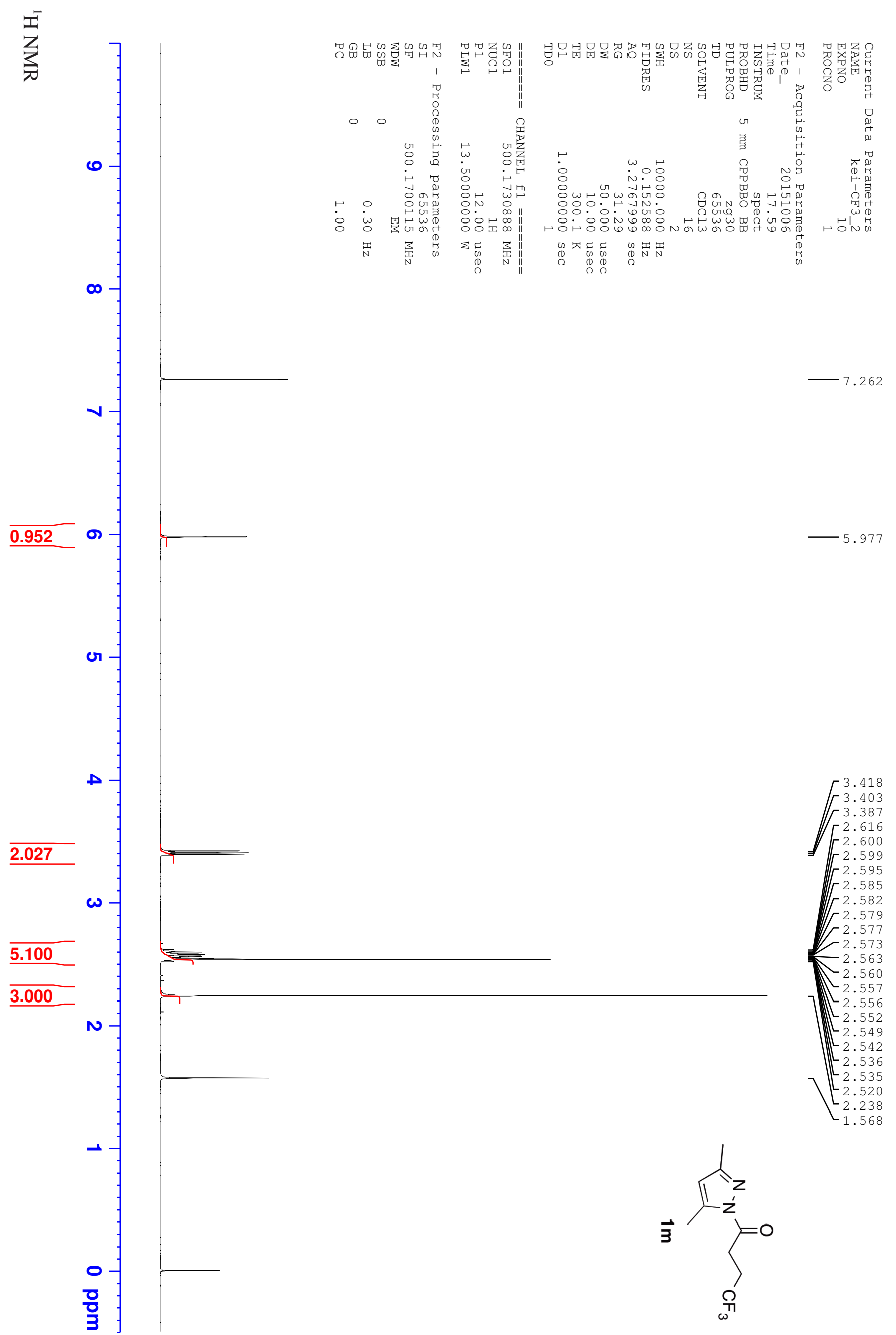


$\overbrace{}^{2}$

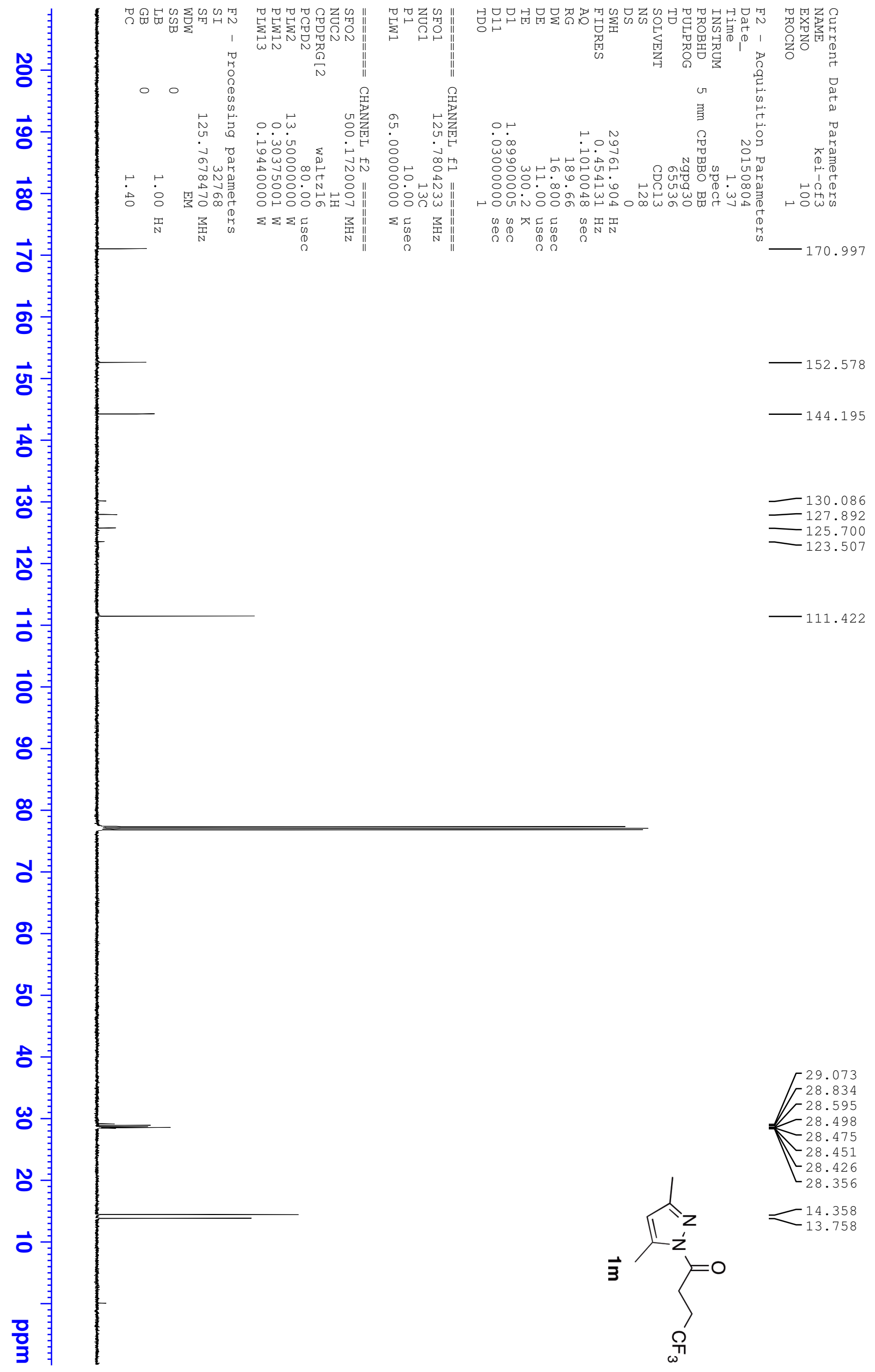




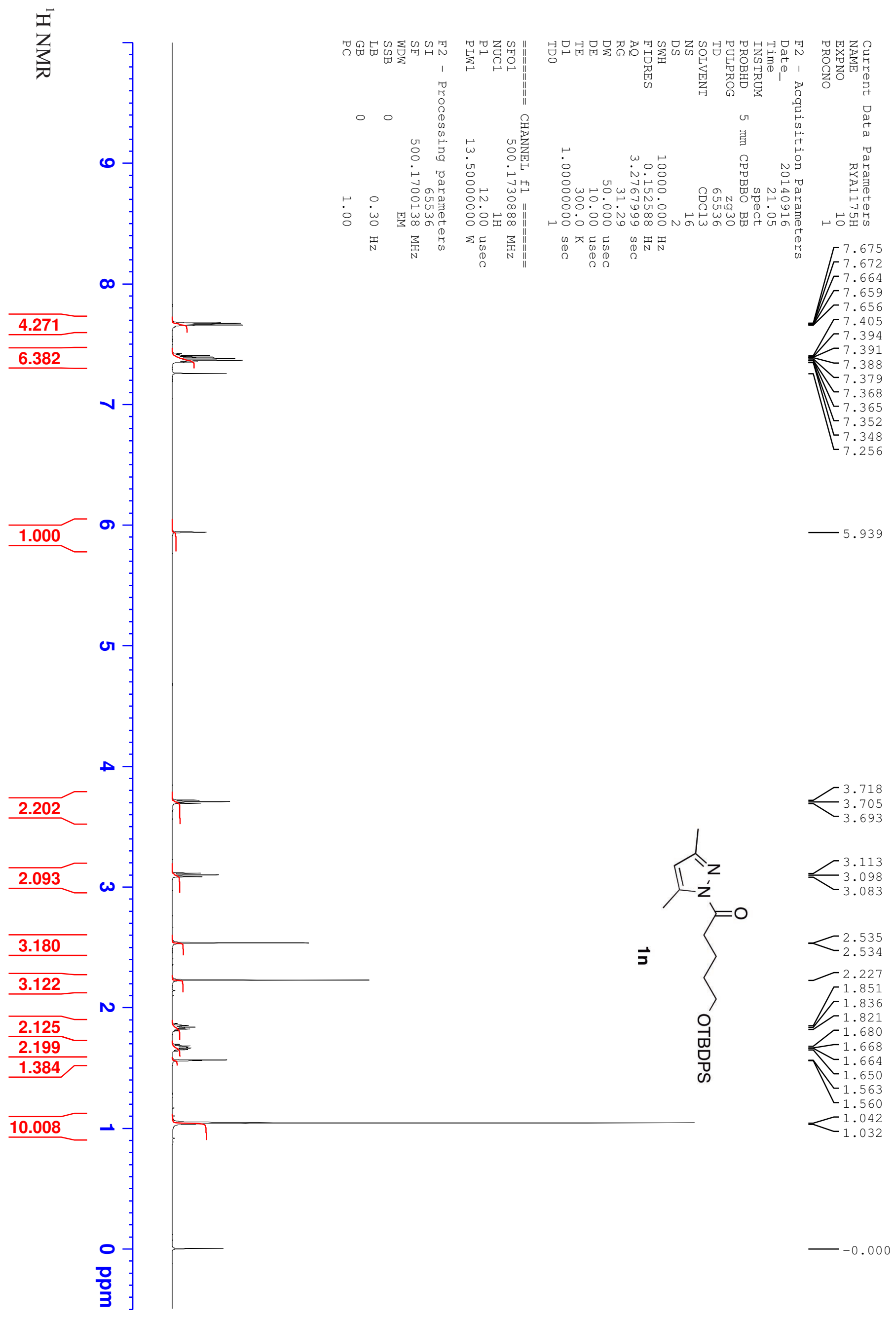


$\overbrace{0}^{\bar{\omega}}$

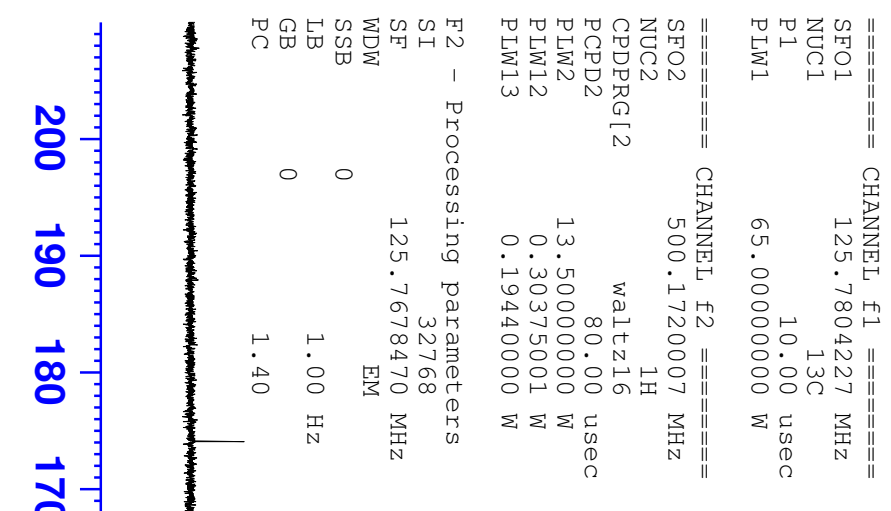

$\begin{array}{lll} & \\ 0 & 0.00\end{array}$

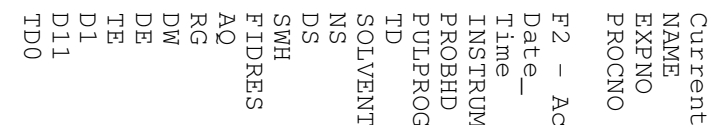

174.018

$\vec{\sigma}$

ले

뭉

$\vec{\omega}$

$\overrightarrow{\tilde{\nu}}$

$\vec{\Delta}$

$\overrightarrow{8}$

8

ஃ

㖫

8

엉

t

$\omega$

ง

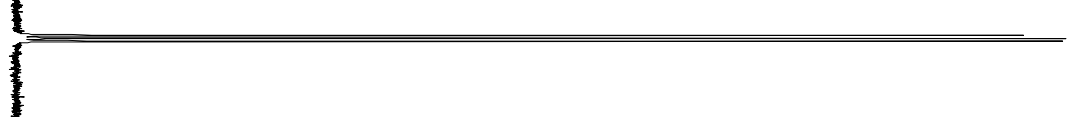

$-151.681$

$-143.948$

$-135.585$

134.024

129.510

$-110.913$

虽

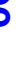

8

韦

青 


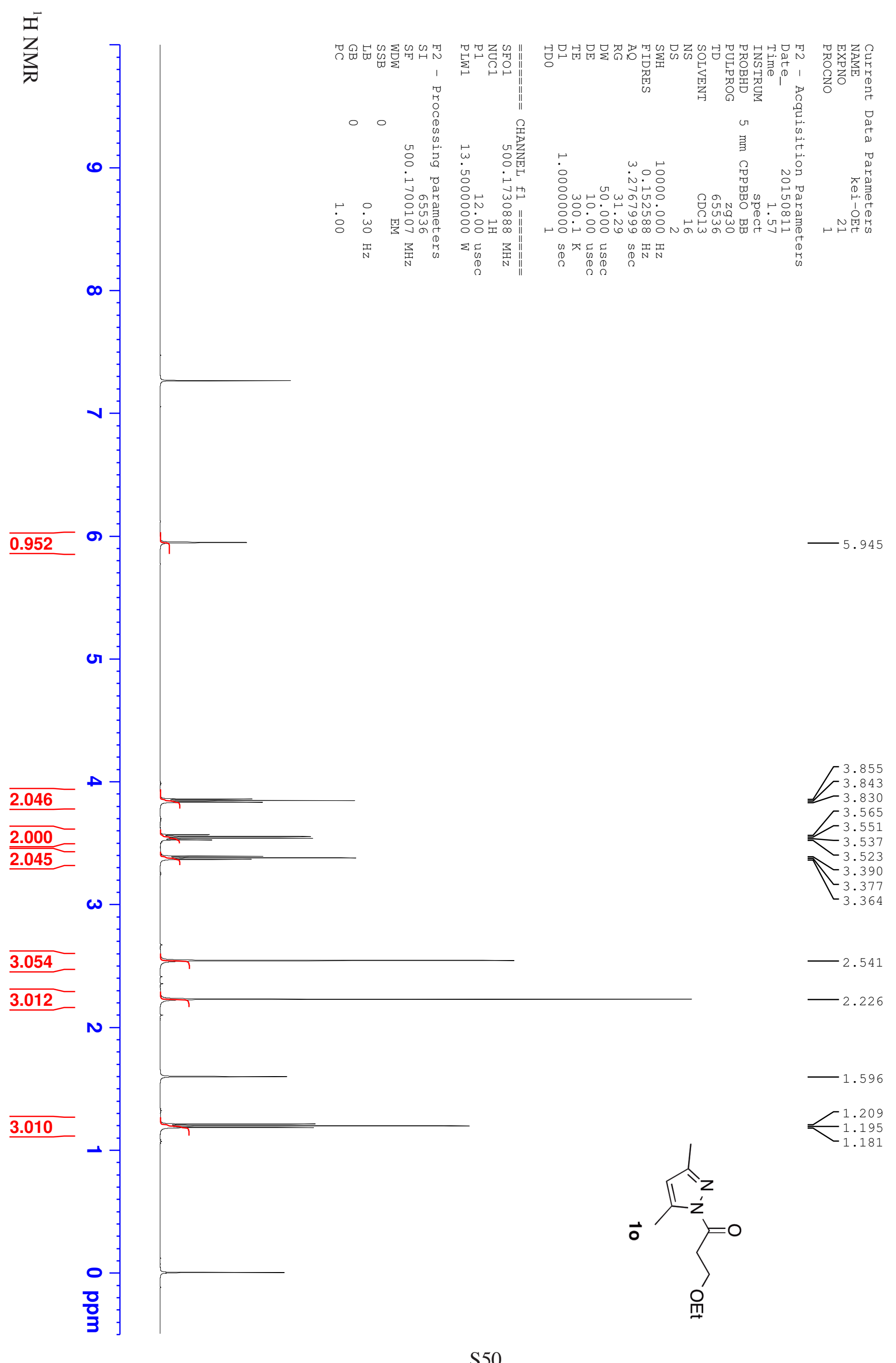


$\overbrace{}^{2}$

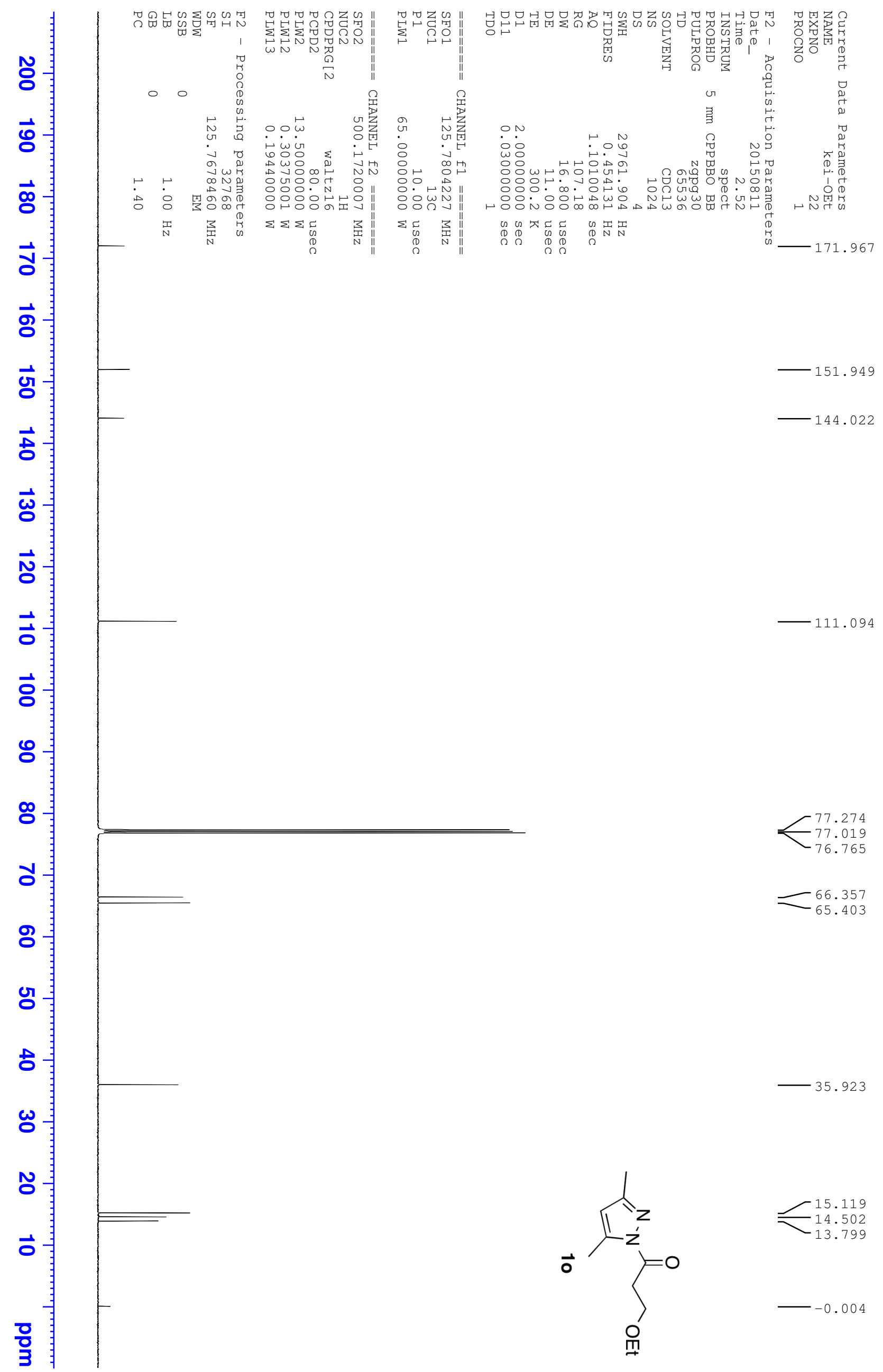




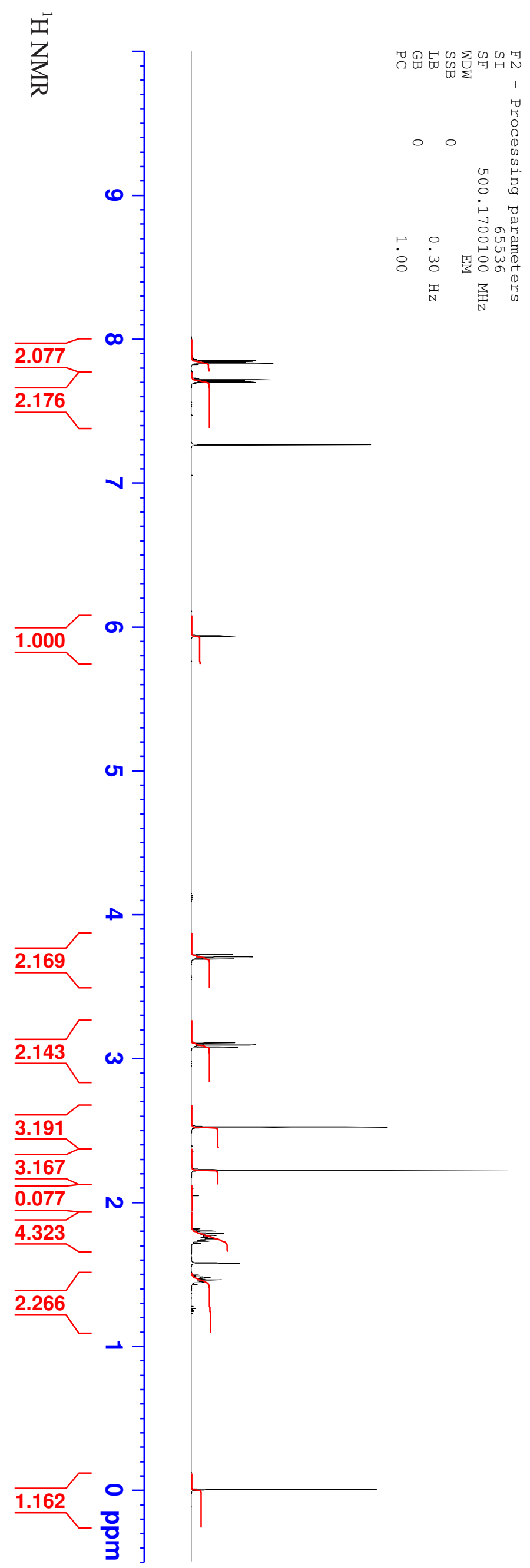

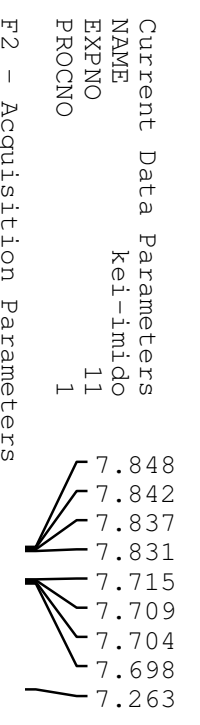

$+5.934$

$-5.932$

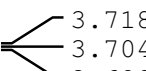

3.704
3.689

3.106 3. 092 3.076

$-2.521$

2.519

2. 222

1.798

1.782

1.767
-1.744

$-1.575$

1.574

1.459

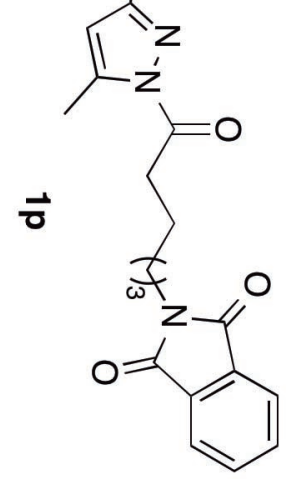
$-0.262$ $-0.350$ 
$\stackrel{2}{2}^{2}$

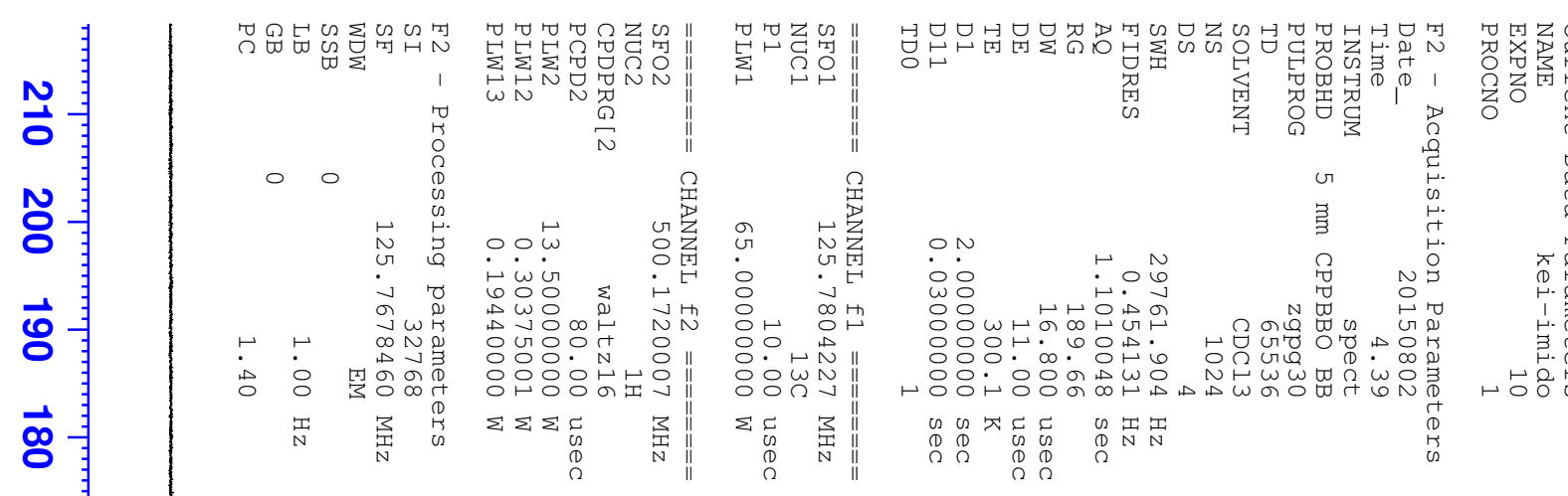

式青

$-173.822$

168.428

$\vec{\circ}$

ज्ञ

$\vec{t}$

$\vec{\omega}$

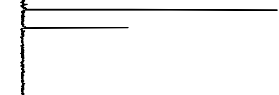

$-133.852$

132.190

$-123.624$

123.183

$\vec{O}$

$\vec{\partial}$

$\overrightarrow{8}$

8

ø

o

8

잉

\&

$\omega$

잉

o

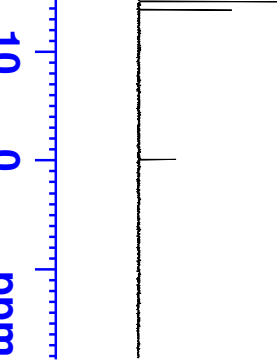

$-110.966$

$-77.272$

77.018

76.764

$-37.864$

35.028

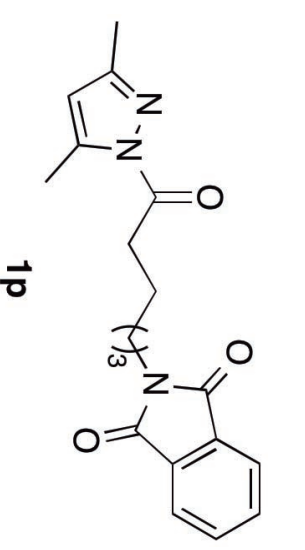

28.450
-26.421

$\simeq 14.562$

13.791

$-0.004$ 


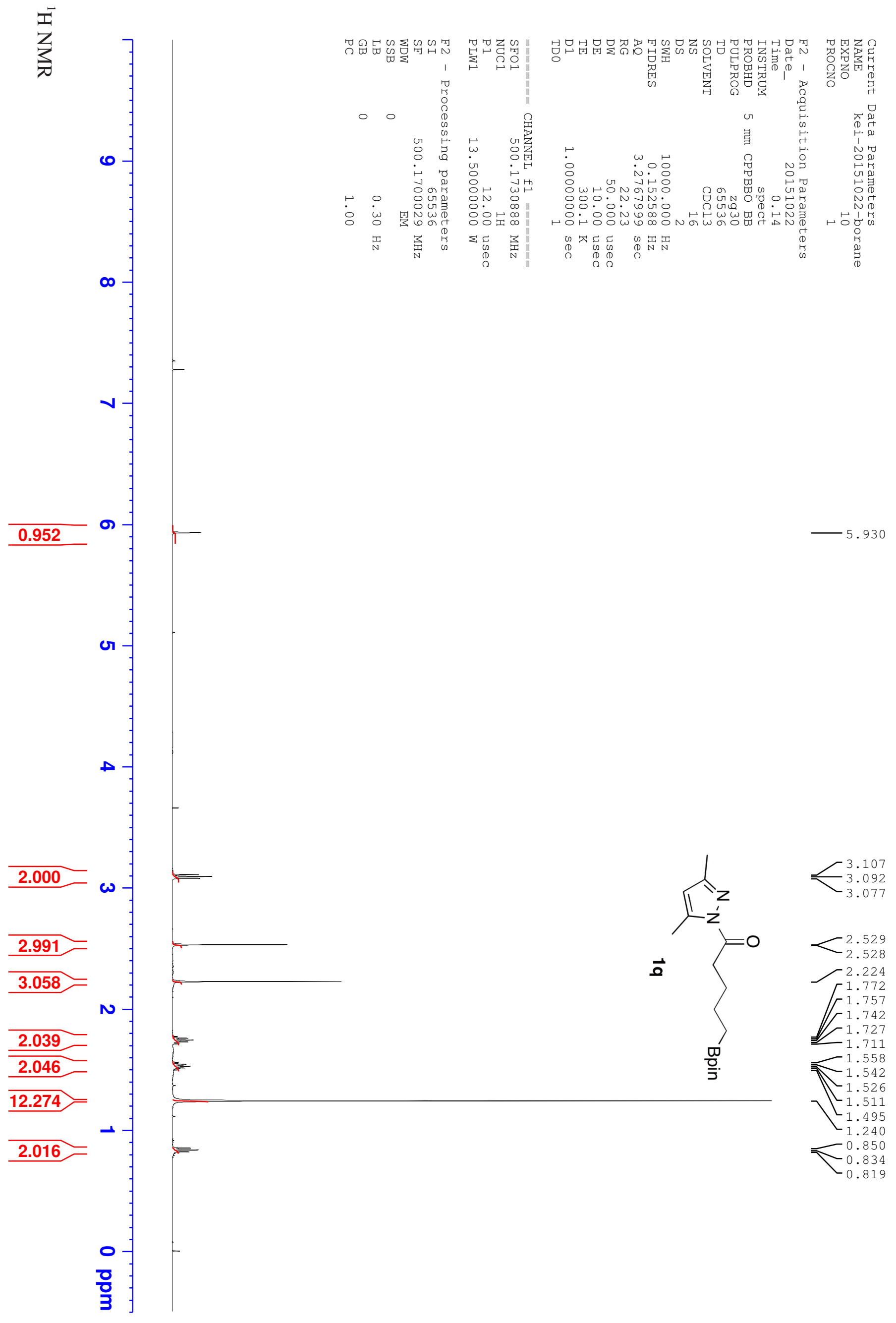


$\overbrace{}^{2}$

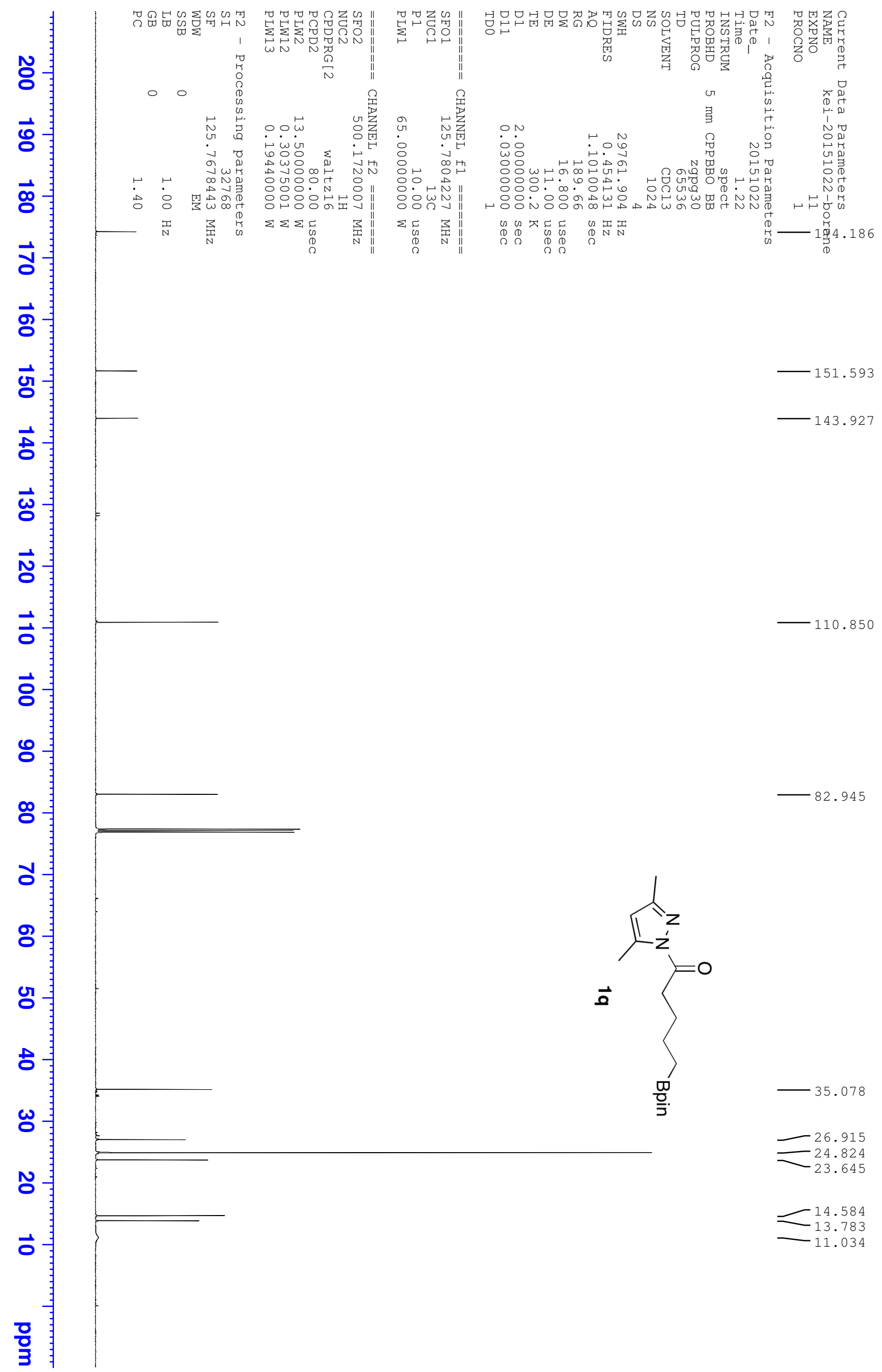




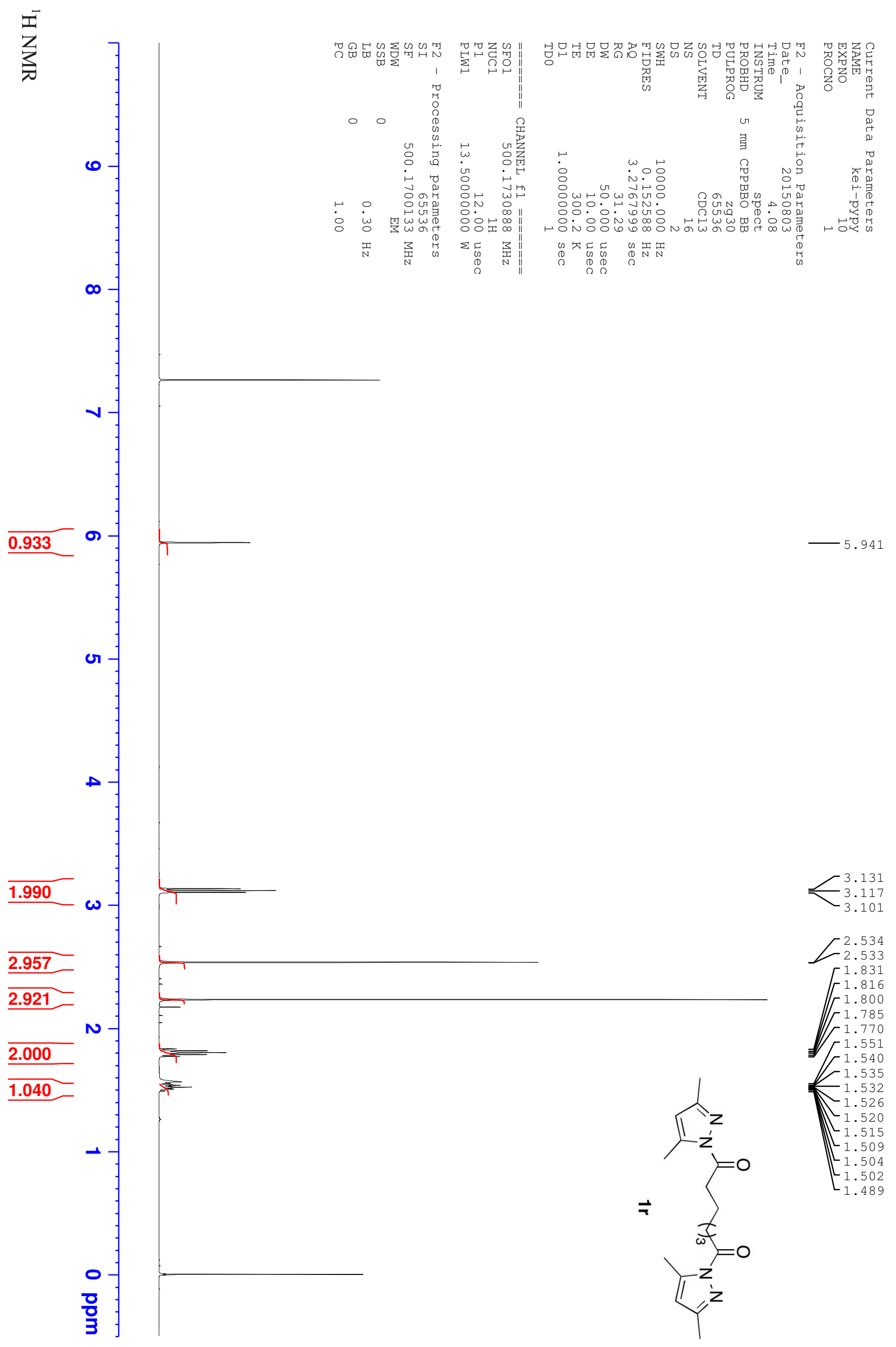


$\overbrace{}^{2}$

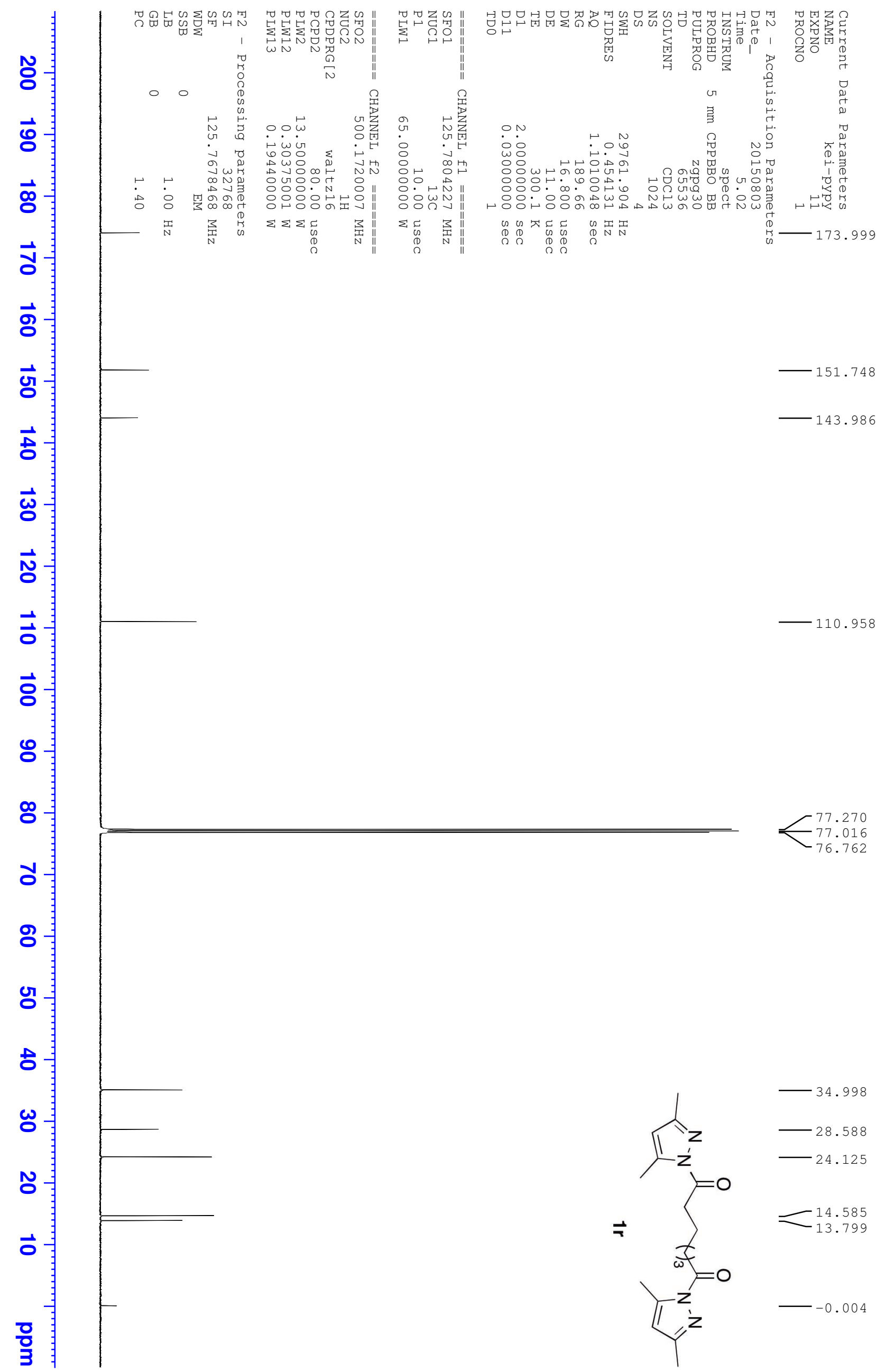




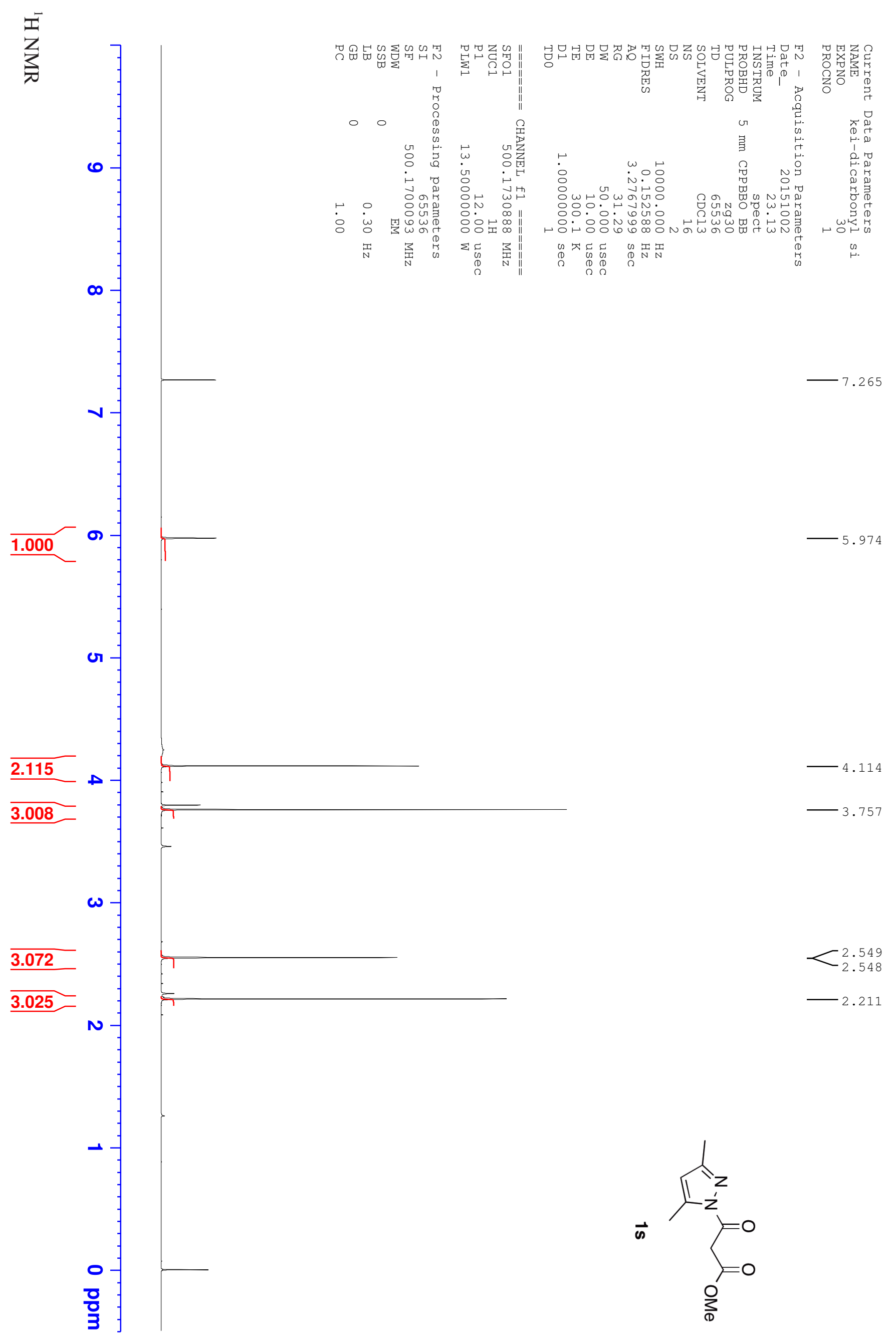


$\overbrace{}^{3}$

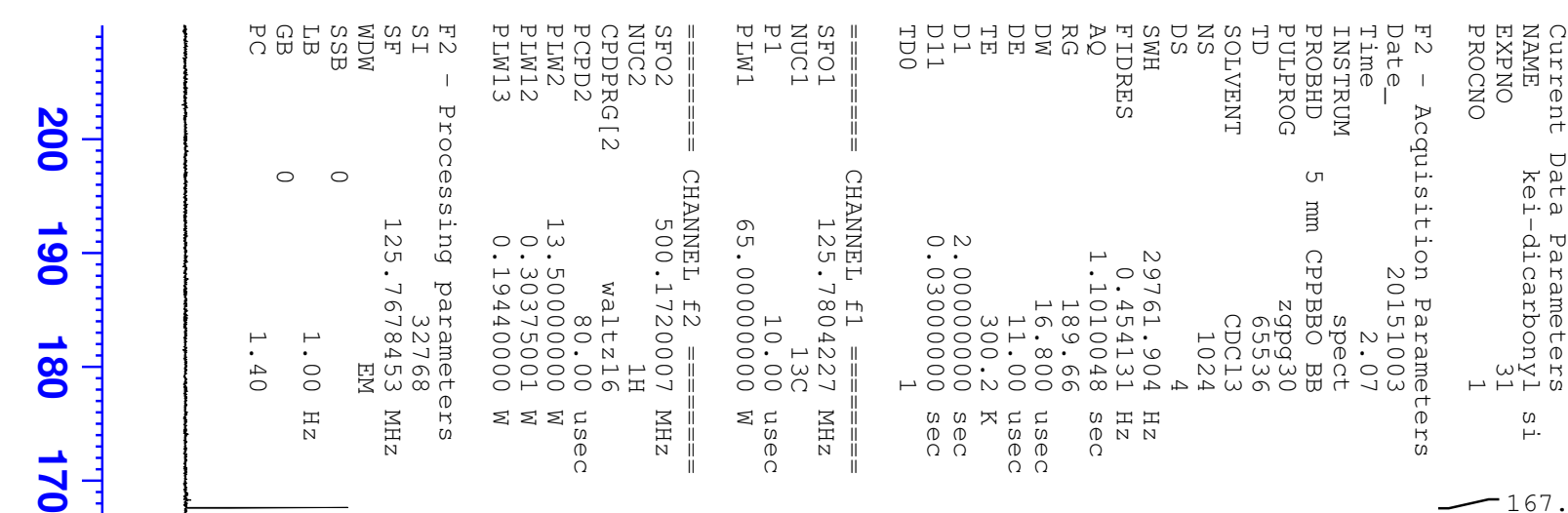

aे

홍

방

$\overrightarrow{\mathrm{t}}$

$\vec{\omega}$

$\overrightarrow{0}$

$\overrightarrow{0}$

$\overrightarrow{8}$

8

๓

¿

8

잉

\&

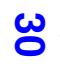

ก
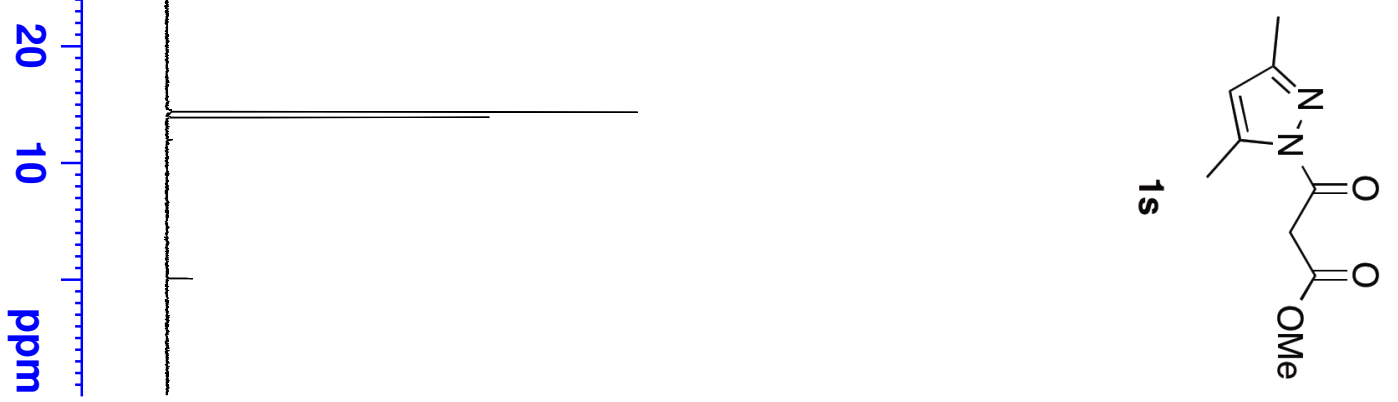

$-52.482$

$-42.840$

$\sim 14.279$ 


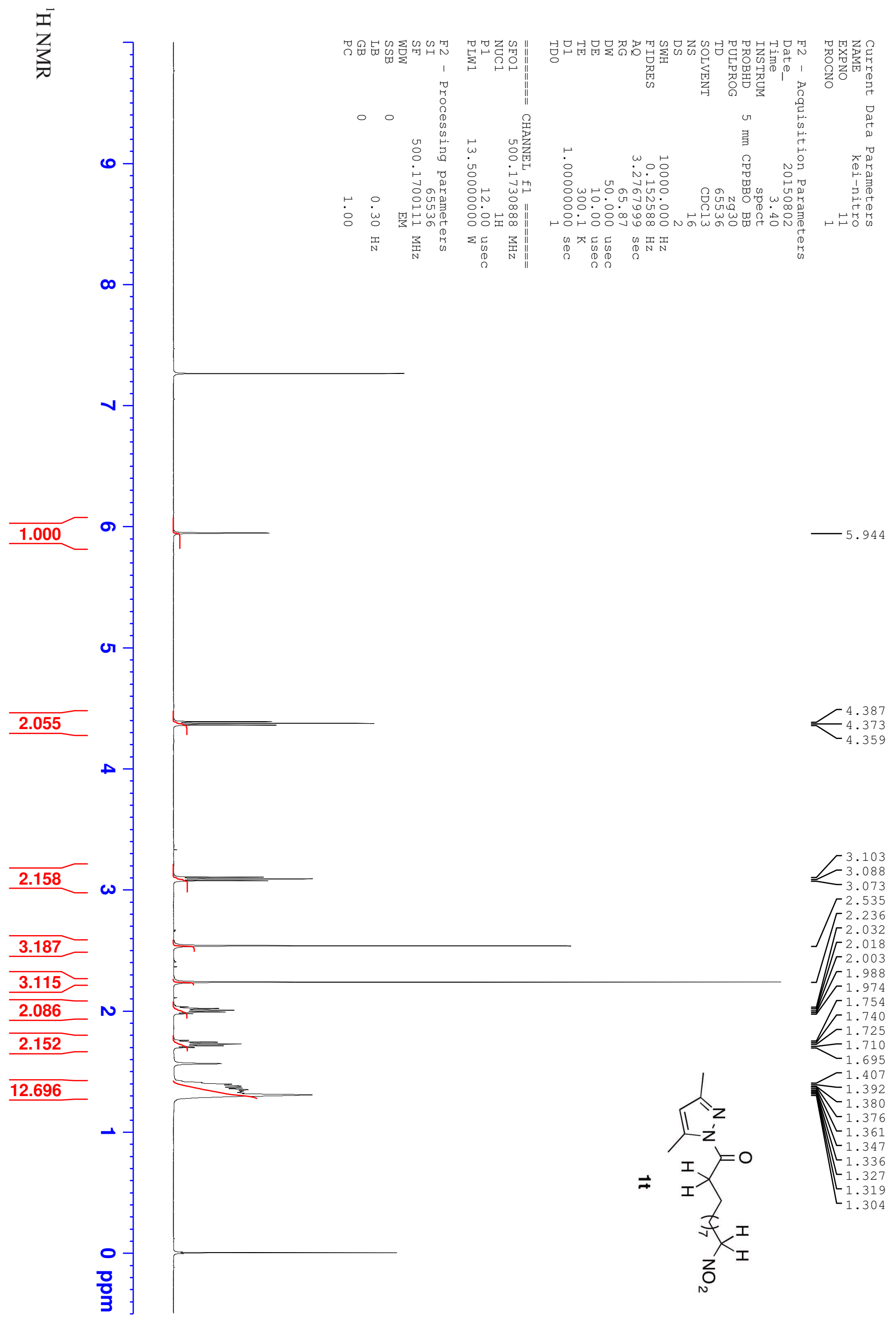


$\overbrace{}^{2}$

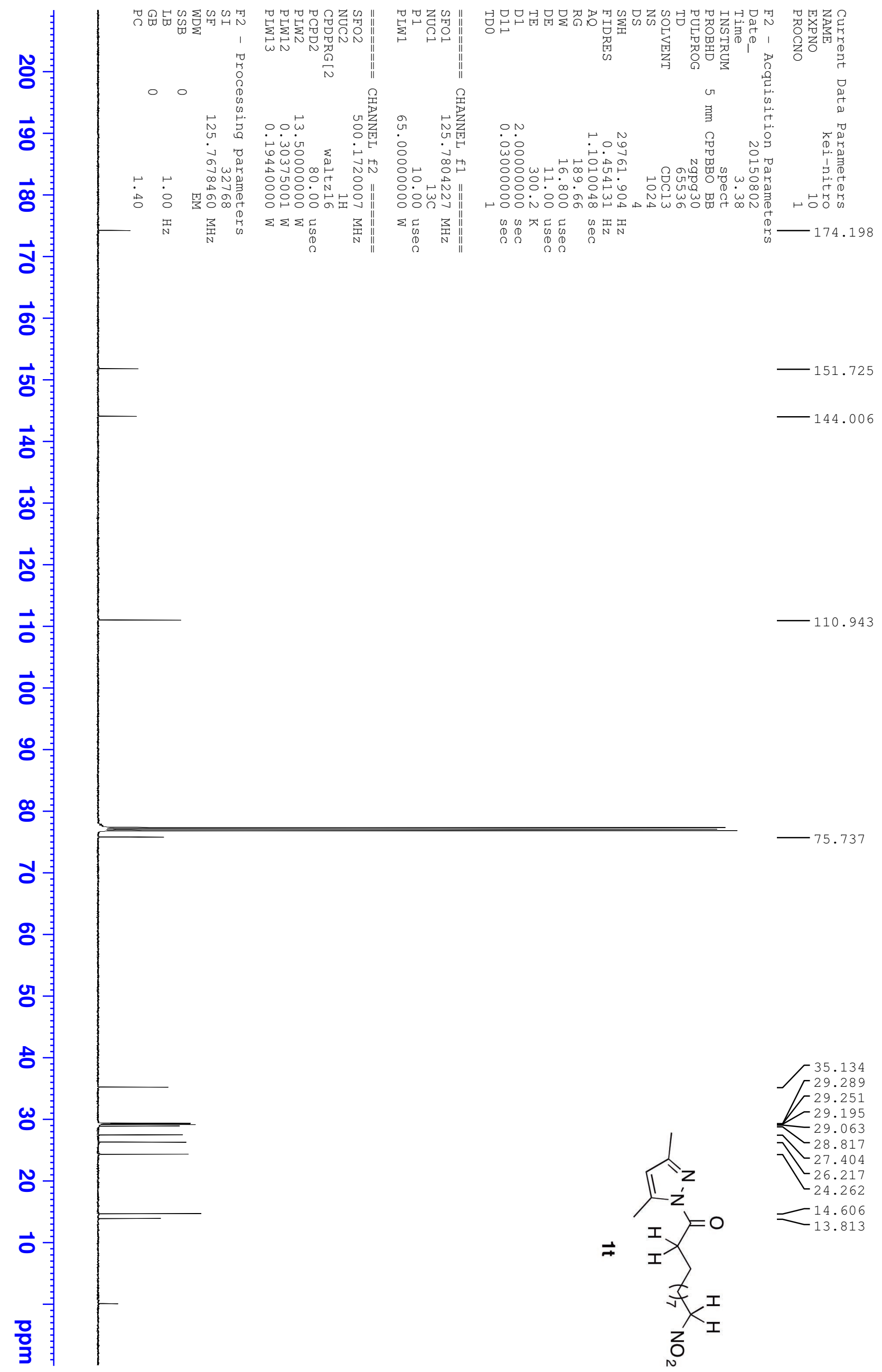




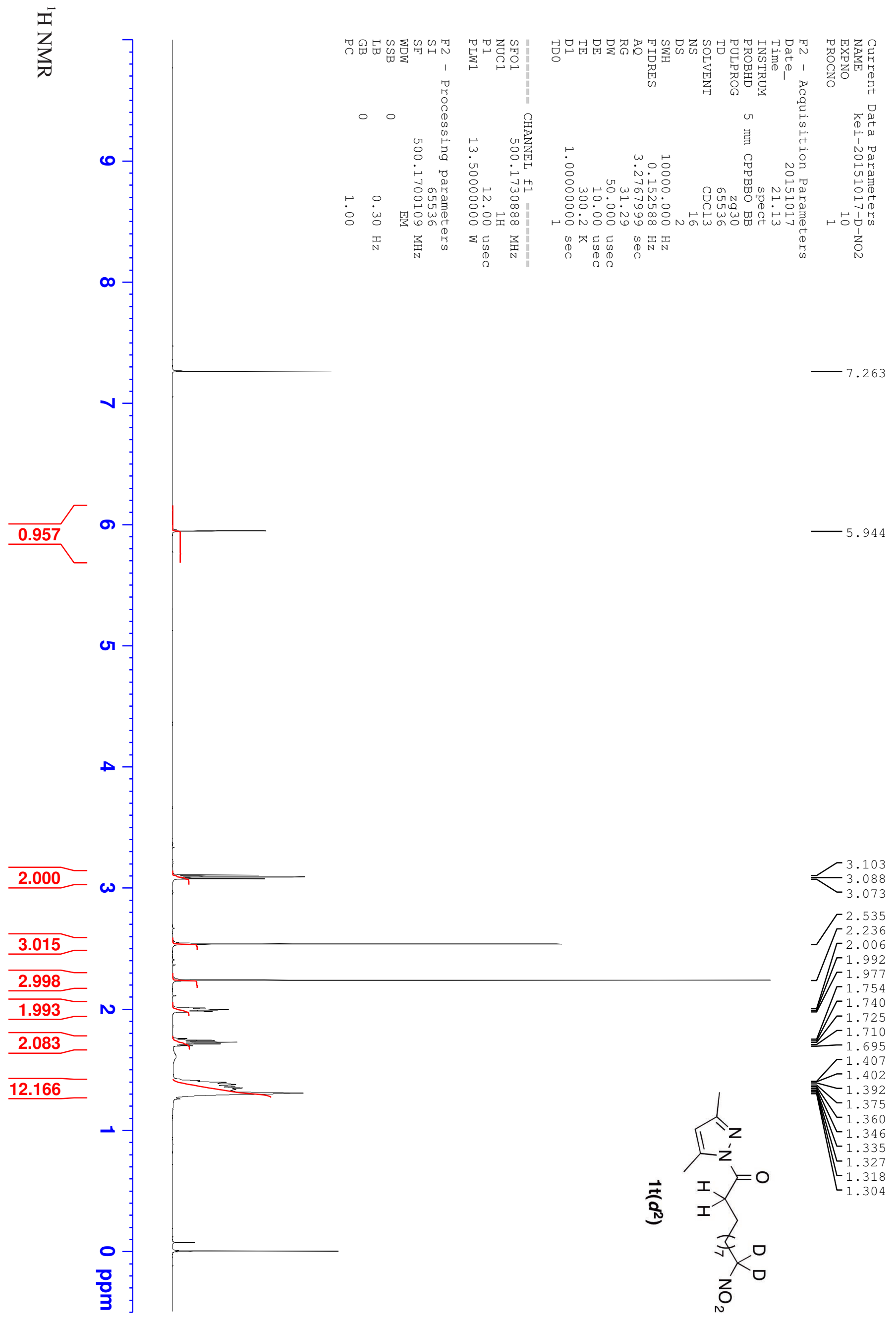


$\overbrace{}^{2}$

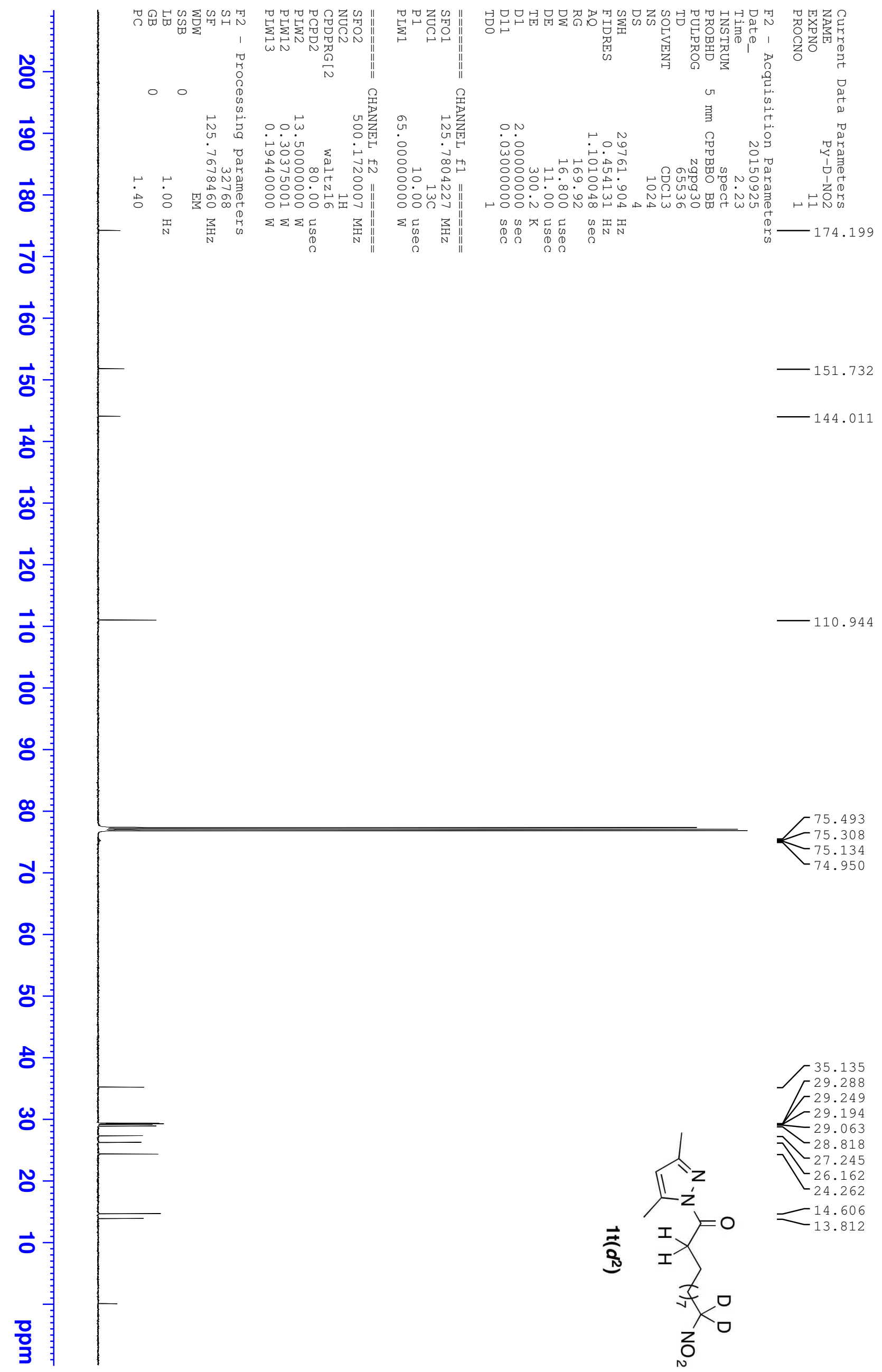



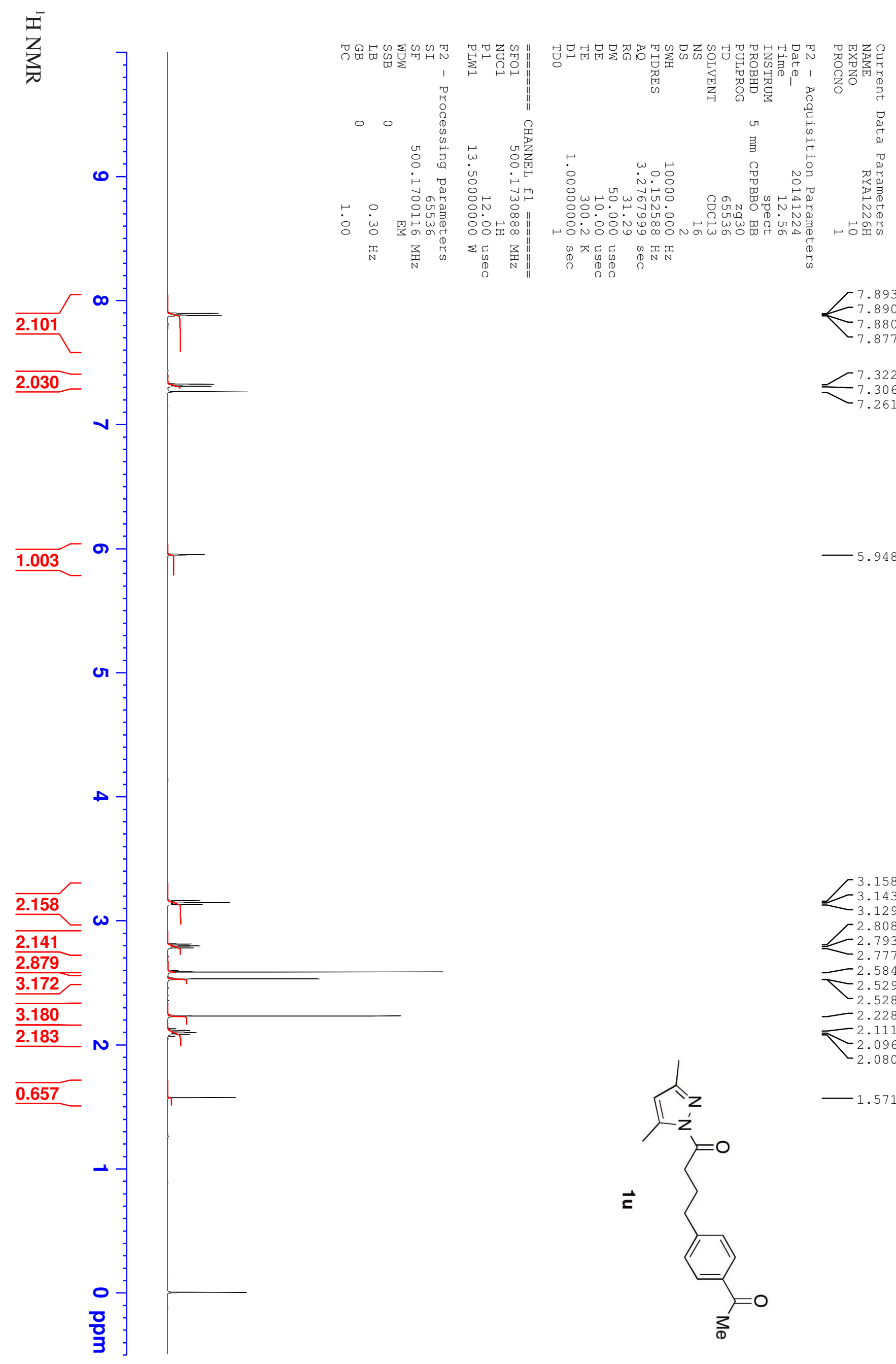

7.893

$-7.890$

7.880
7.877

7.322

7.306

3.129

2.808
-2.793

$-2.77$

2.584
-2.529

2.528

2.228

$-2.111$

$-2.096$

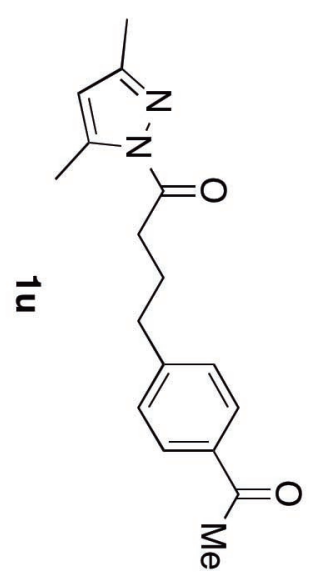

2.080 
$\overbrace{}^{2}$

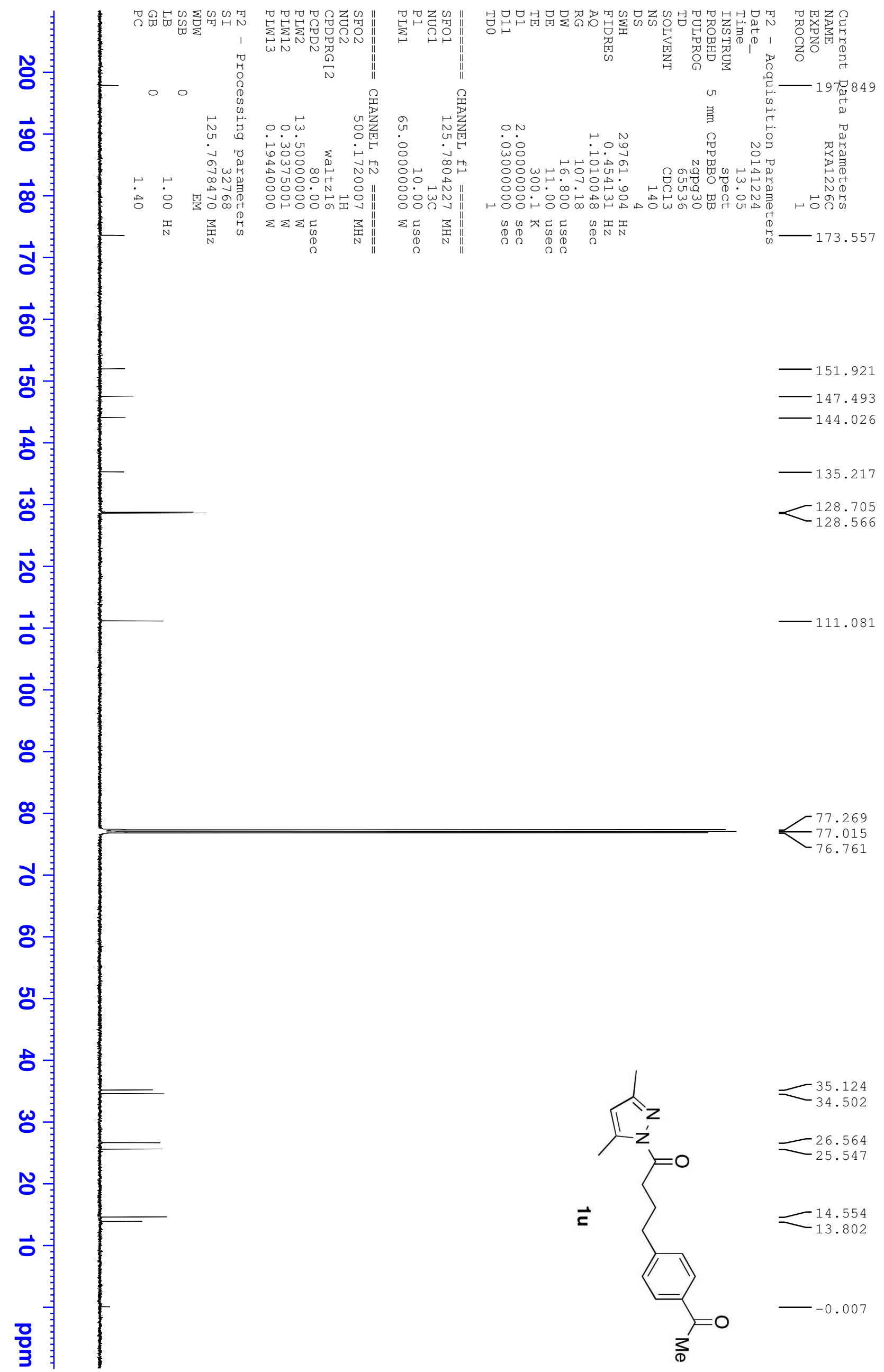




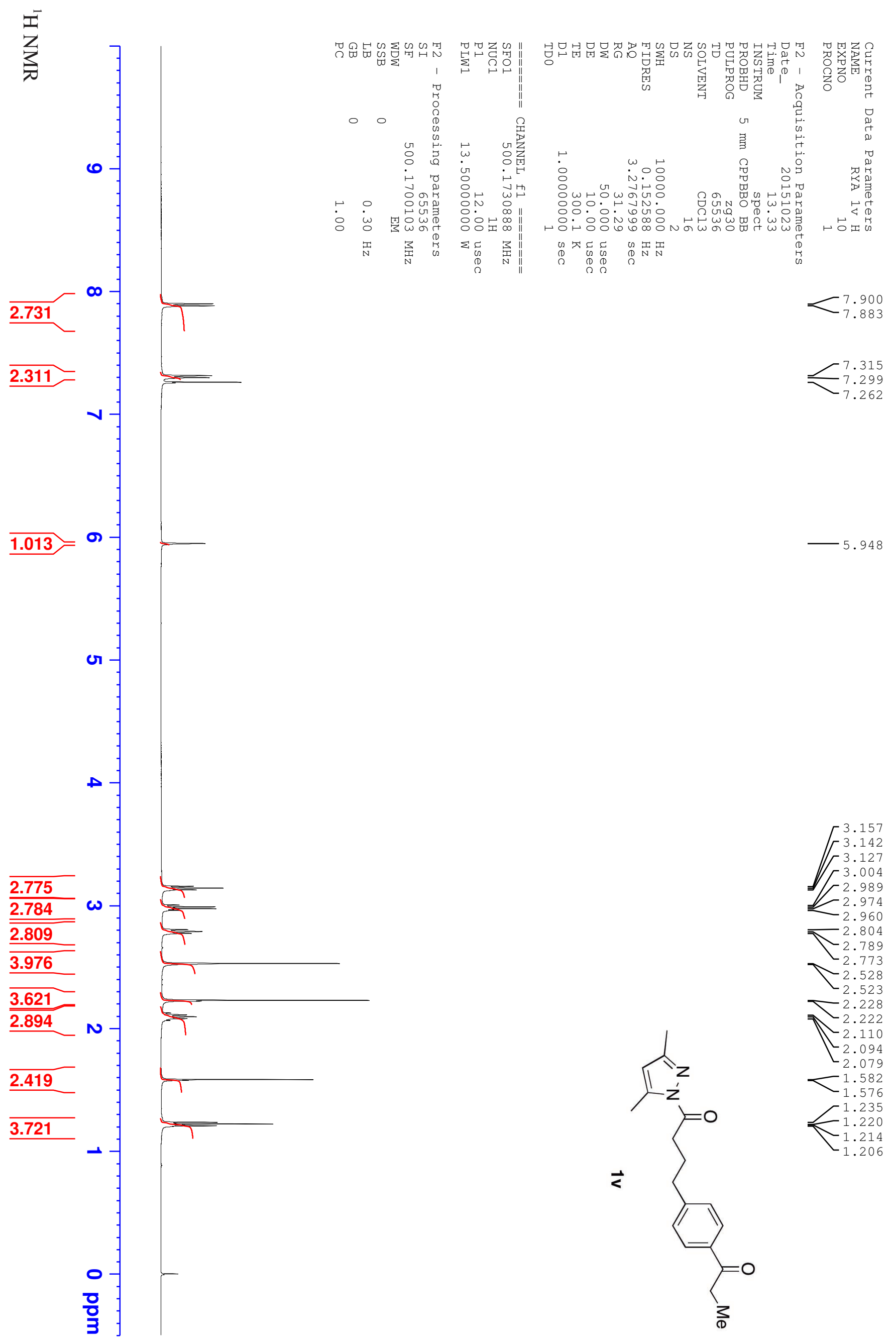


$\overbrace{}^{\bar{\omega}}$

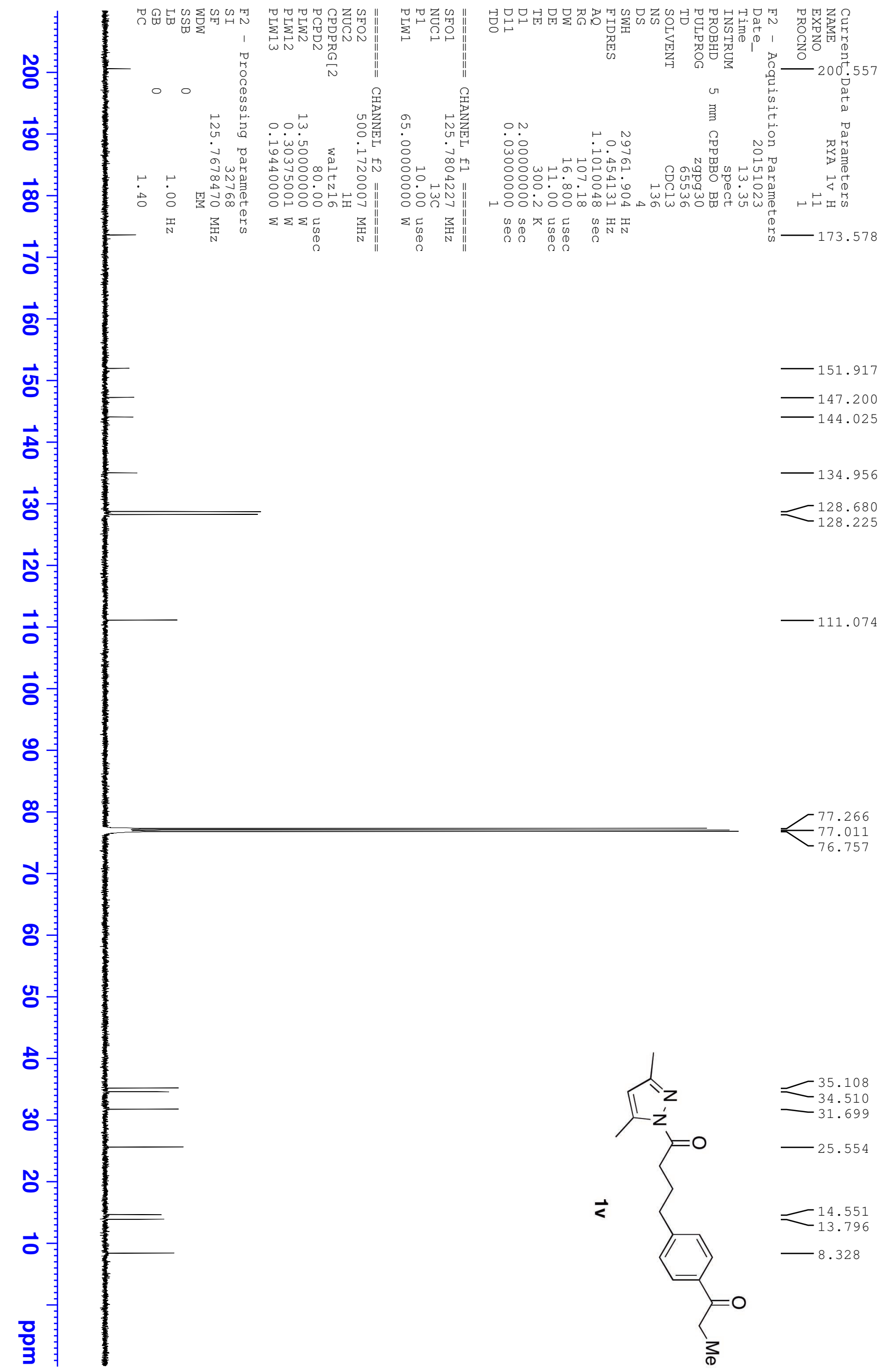



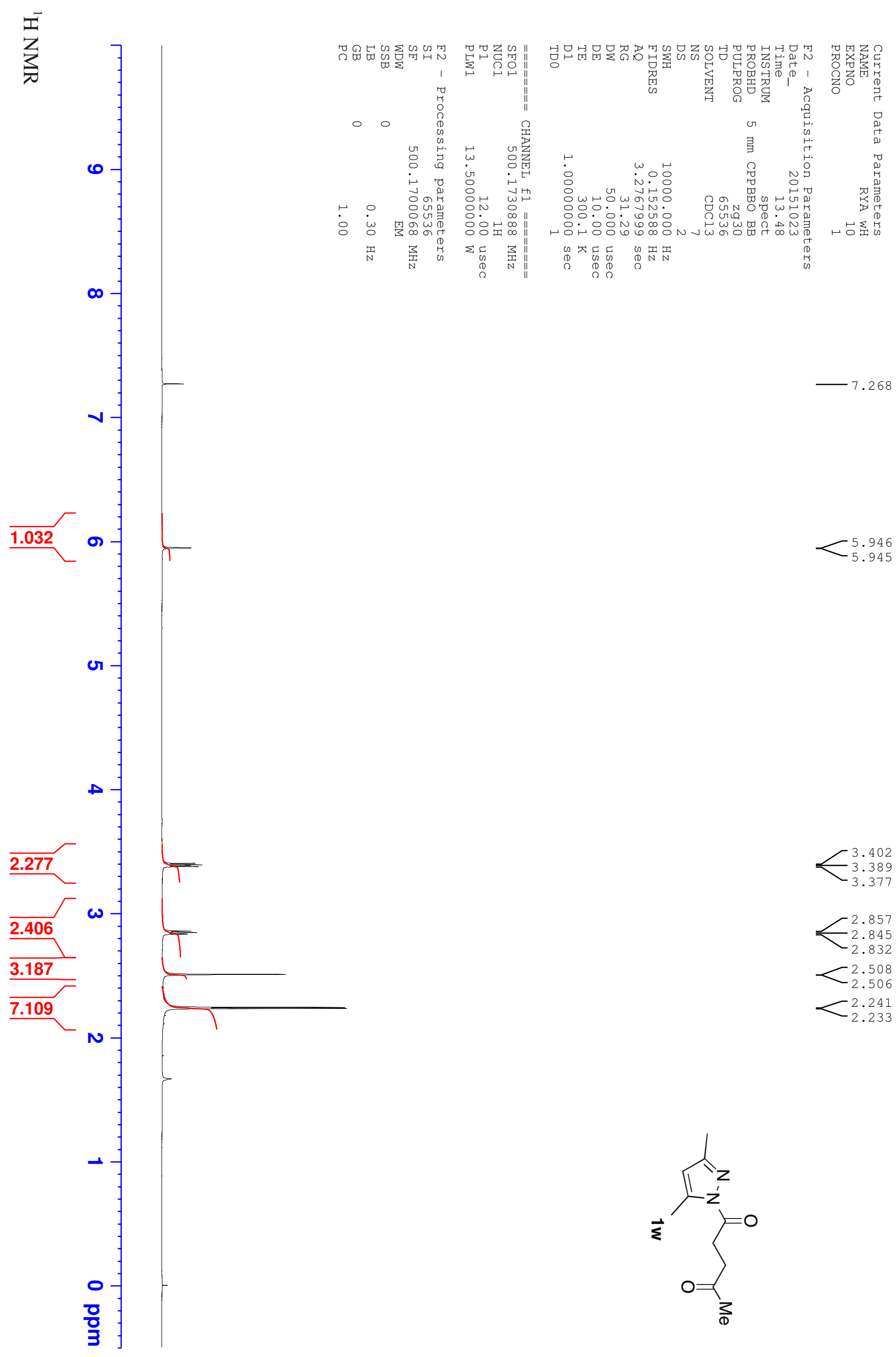

2.857

2.845

2.832

$-2.508$

$-2.506$

$<2.241$

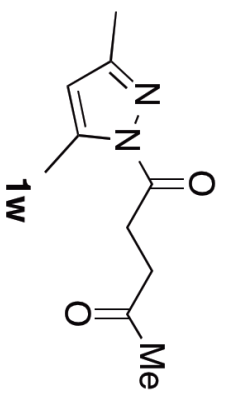


$\overbrace{}^{\bar{\omega}}$

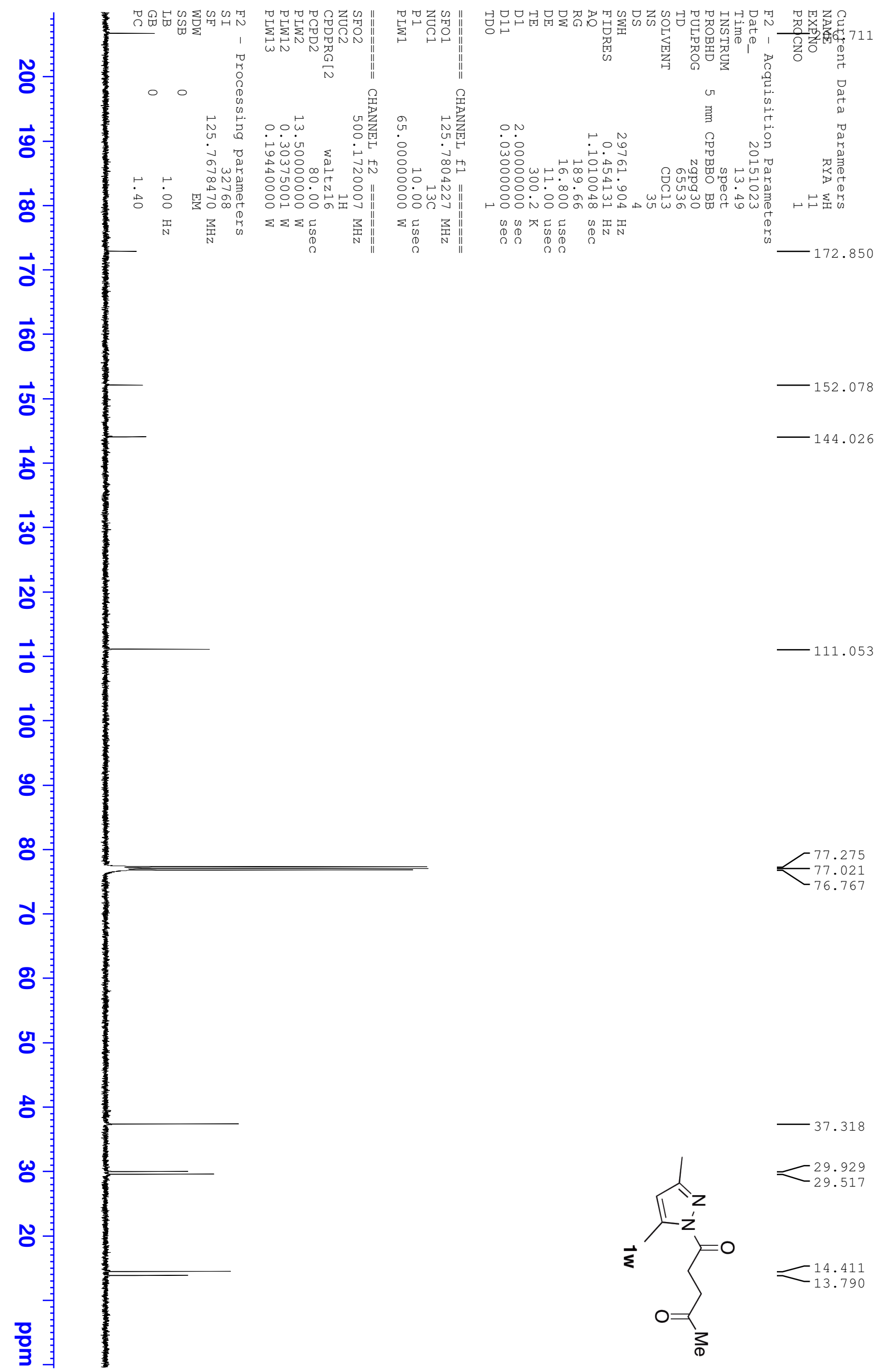




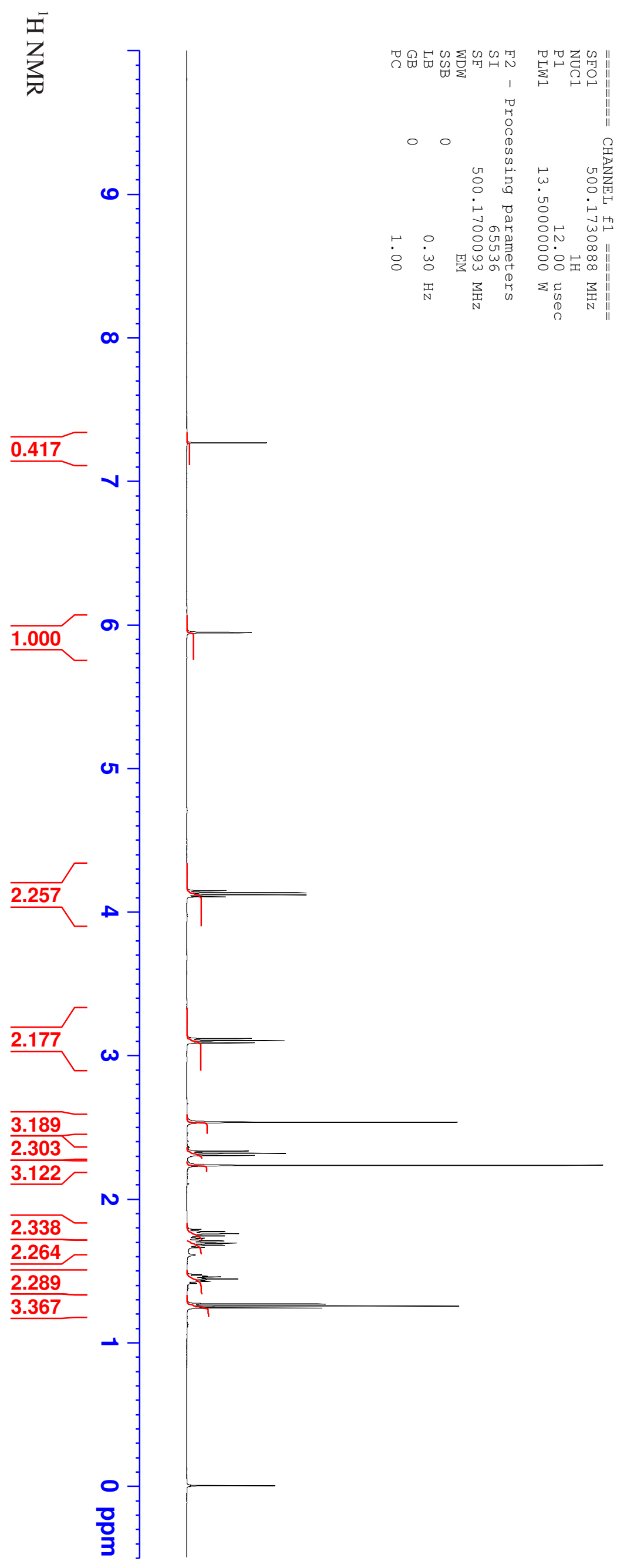

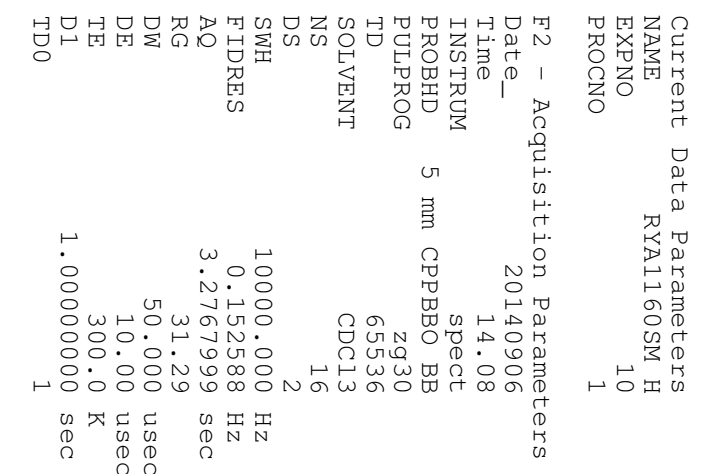

r.145

4. 131

4.117

4.102

3.115

3.100

2.533

$\digamma^{2.531}$

$r^{2.331}$

2.300

2.232

1.771

1.756

$F^{1.740}$

1.706
-1.690

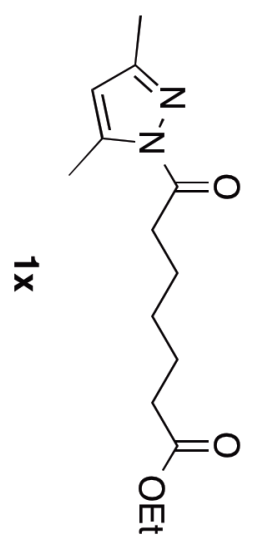

$-1.675$

$-1.460$

$-1.455$

$-1.453$

$\left[\begin{array}{l}1.440 \\ 1.425\end{array}\right.$

$[1.425$

1.266

1.256

$\left[\begin{array}{l}1.252 \\ 1.238\end{array}\right.$ 
$\overbrace{}^{\bar{\omega}}$

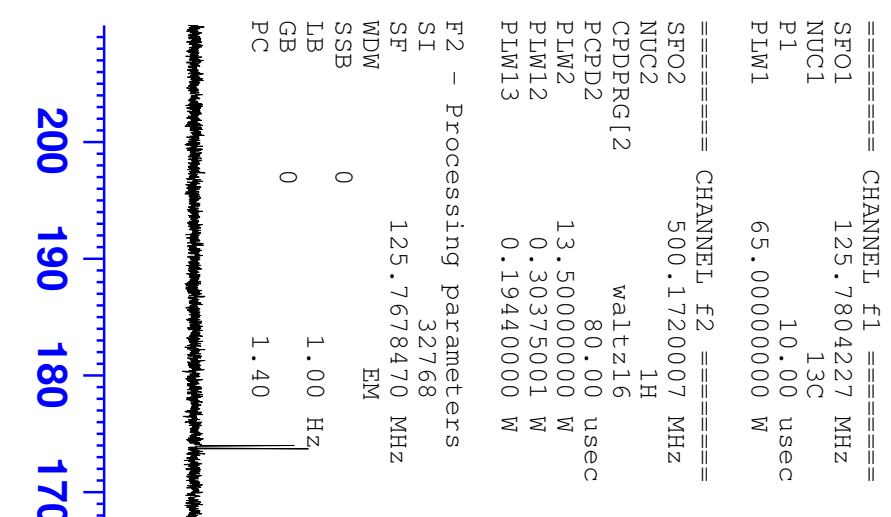

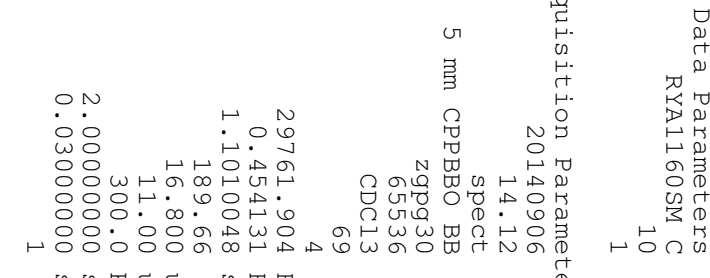

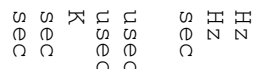

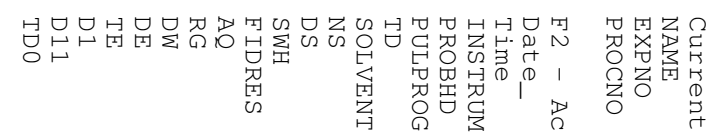

173.927
$-\quad 173.666$

$\vec{\circ}$

ज़

$\overrightarrow{0}$

$\overrightarrow{\text {. }}$

$\overrightarrow{\text { N }}$

$\vec{a}$

웅

๕

$\infty$

$\infty$ 畨

ปั

8

잉

을

$\omega$

ธิ

o

g
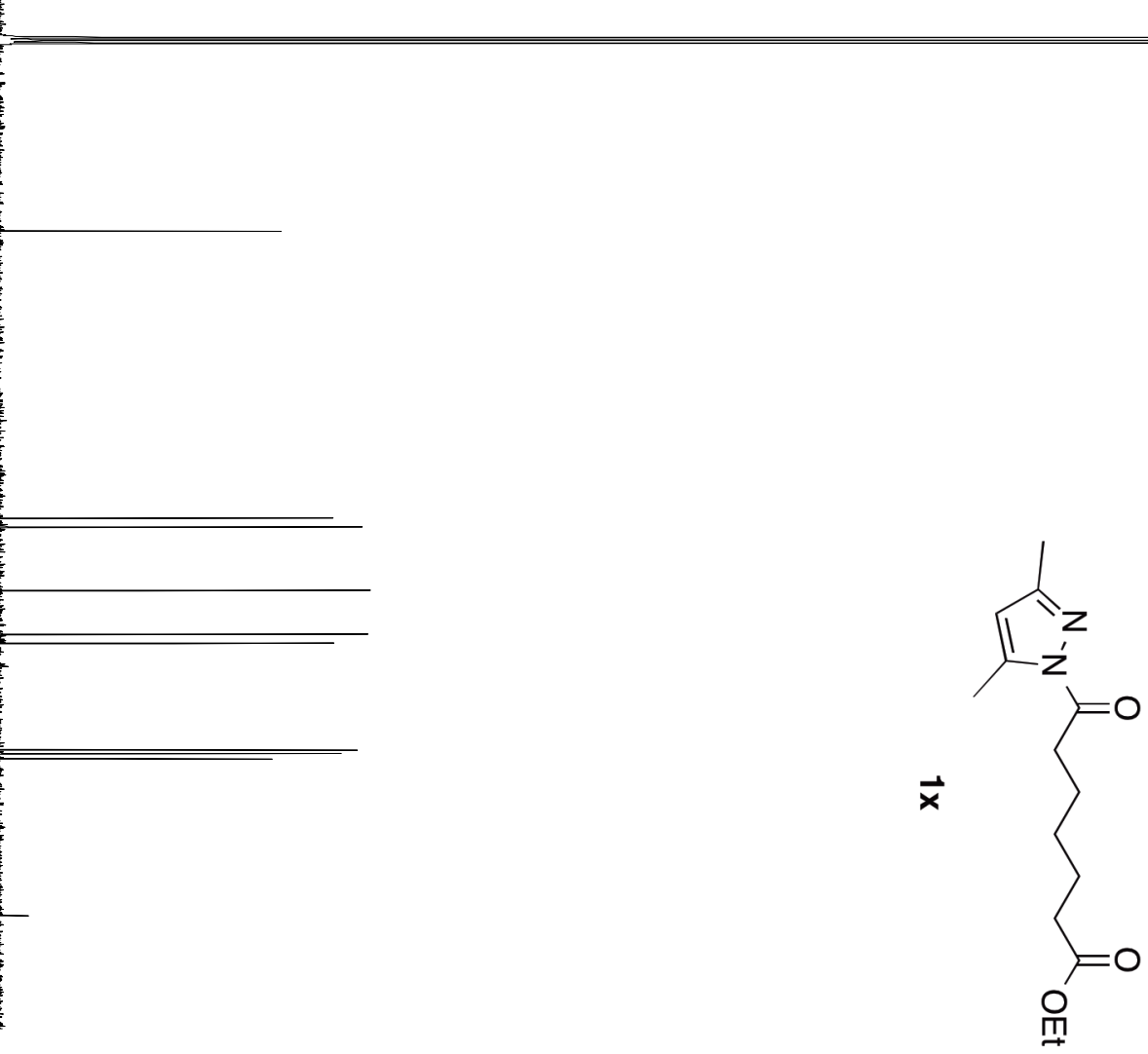

34.950
${ }_{34.184}$

38.587

$\begin{array}{r}28.587 \\ -\quad 24.737 \\ \hline\end{array}$

23.943

$\begin{array}{r}14.569 \\ -14.245 \\ \hline\end{array}$

14.245
13.790

$-0.012$

㽞 


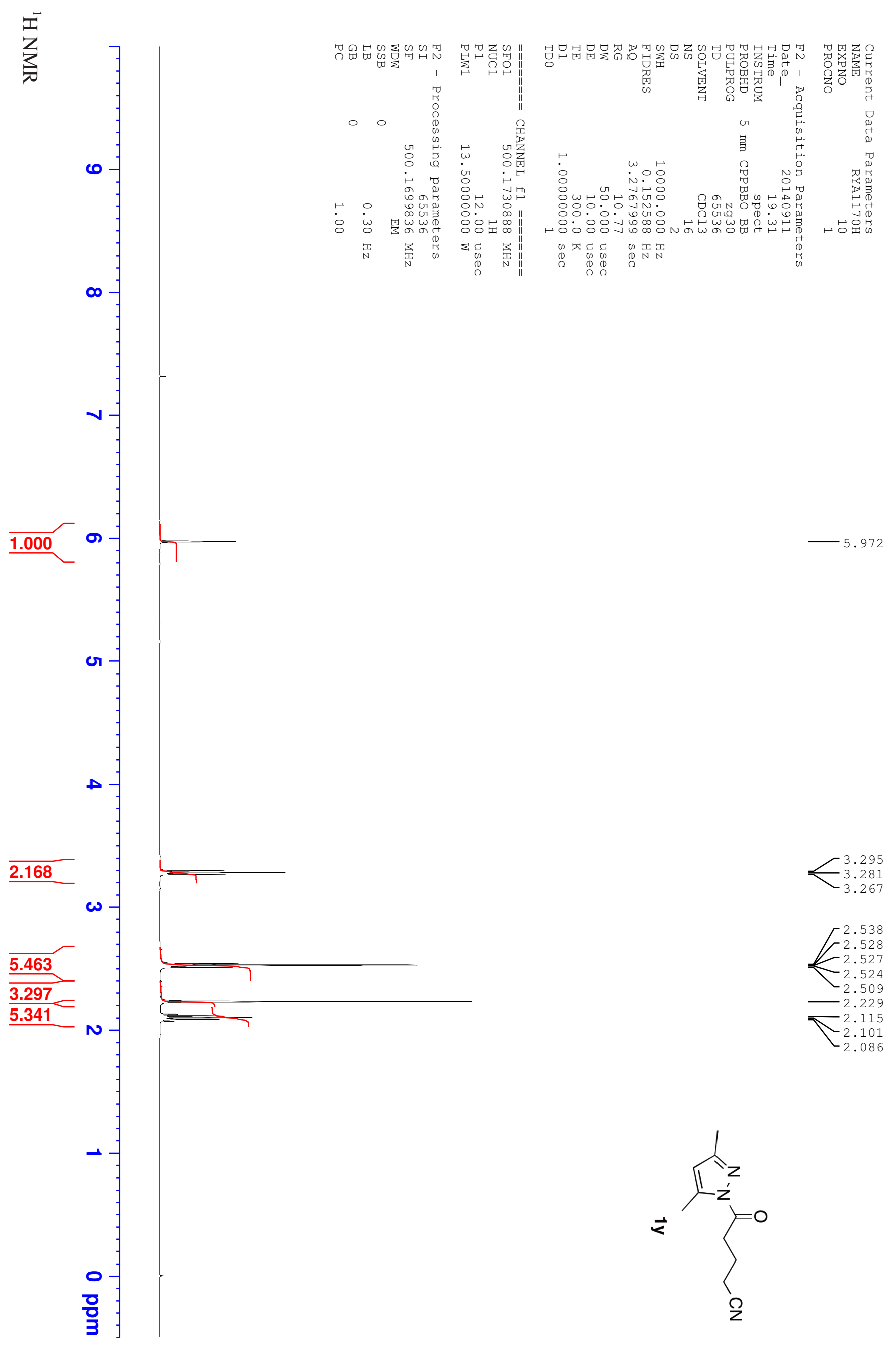


$\overbrace{}^{2}$

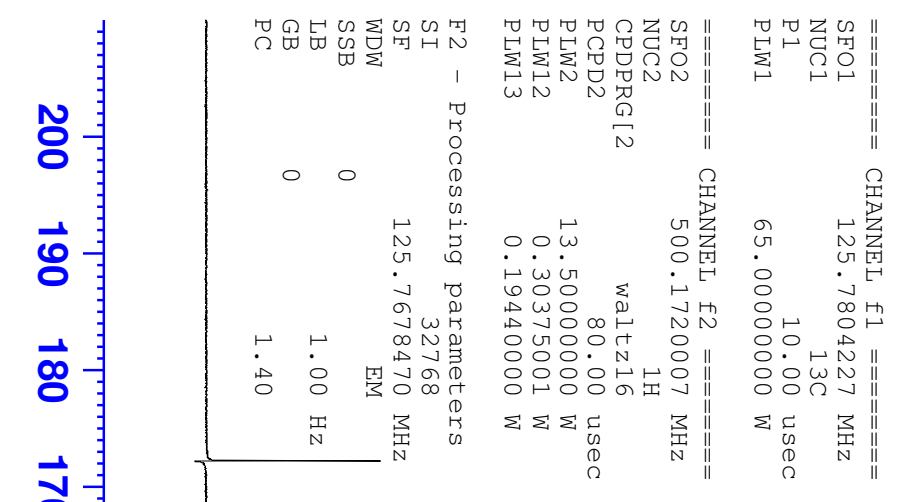

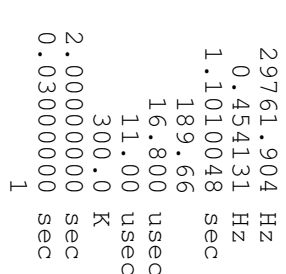

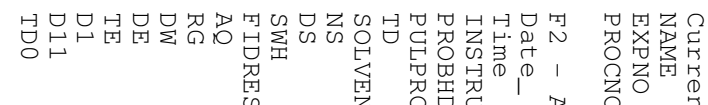

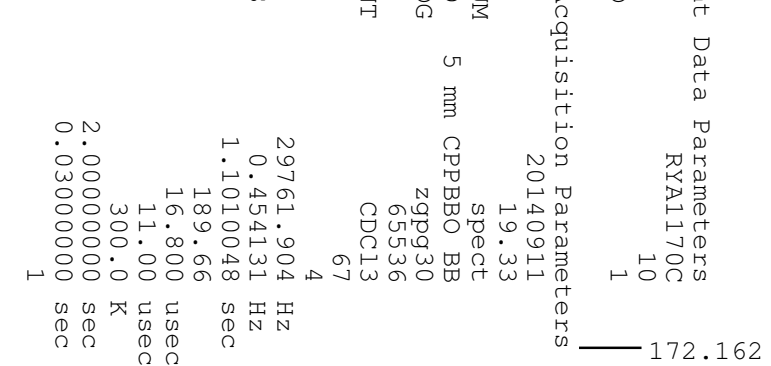

$\overrightarrow{\text { g }}$

ज़

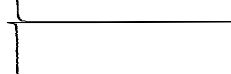

$-152.273$

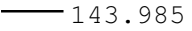

$\overrightarrow{0}$

$\vec{\omega}$

$\overrightarrow{\mathrm{o}}$

$\overrightarrow{0}$

$\overrightarrow{8}$

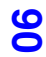

\&

ㄱ

o

\&

잉
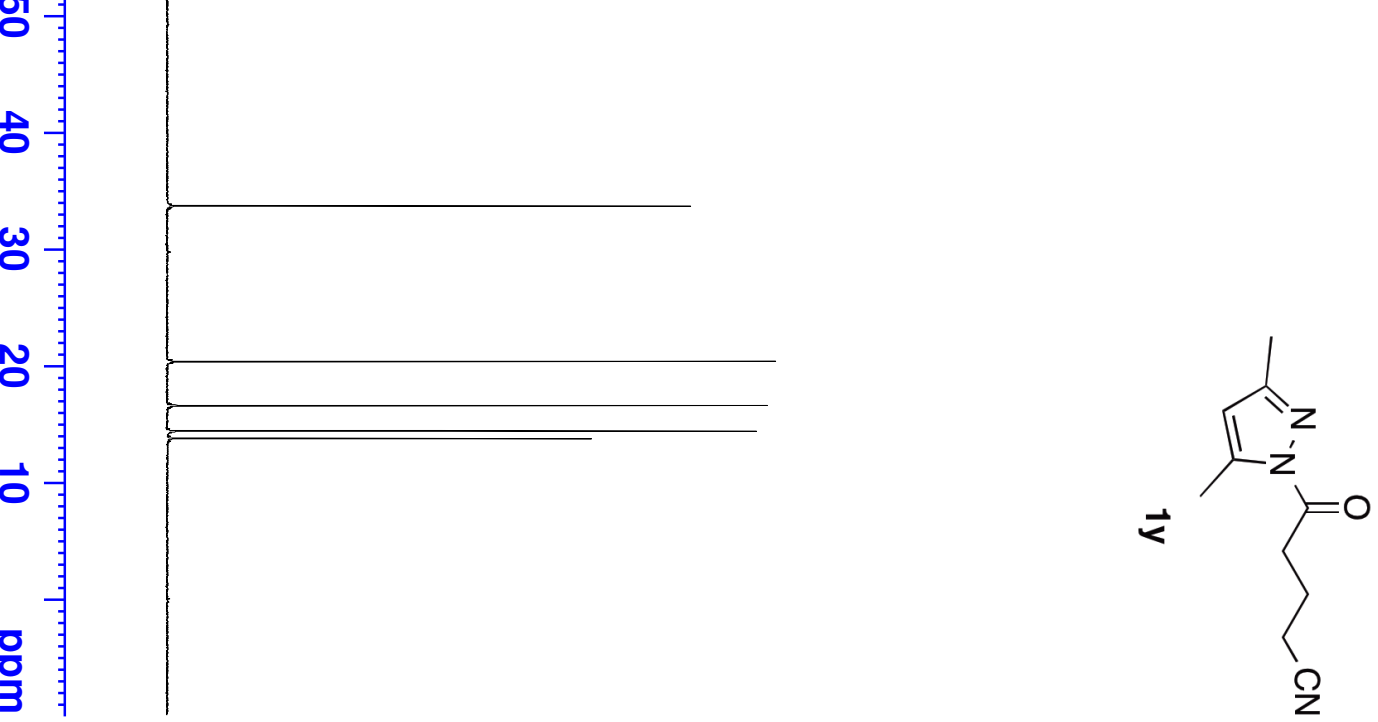

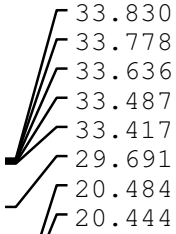

20.311

16.742

16.665

16.541
16.396

$-16.296$

14.543
14.361

14.359
14.159

13.913

$\left[\begin{array}{l}13.716 \\ 13.500\end{array}\right.$

$-0.055$ 


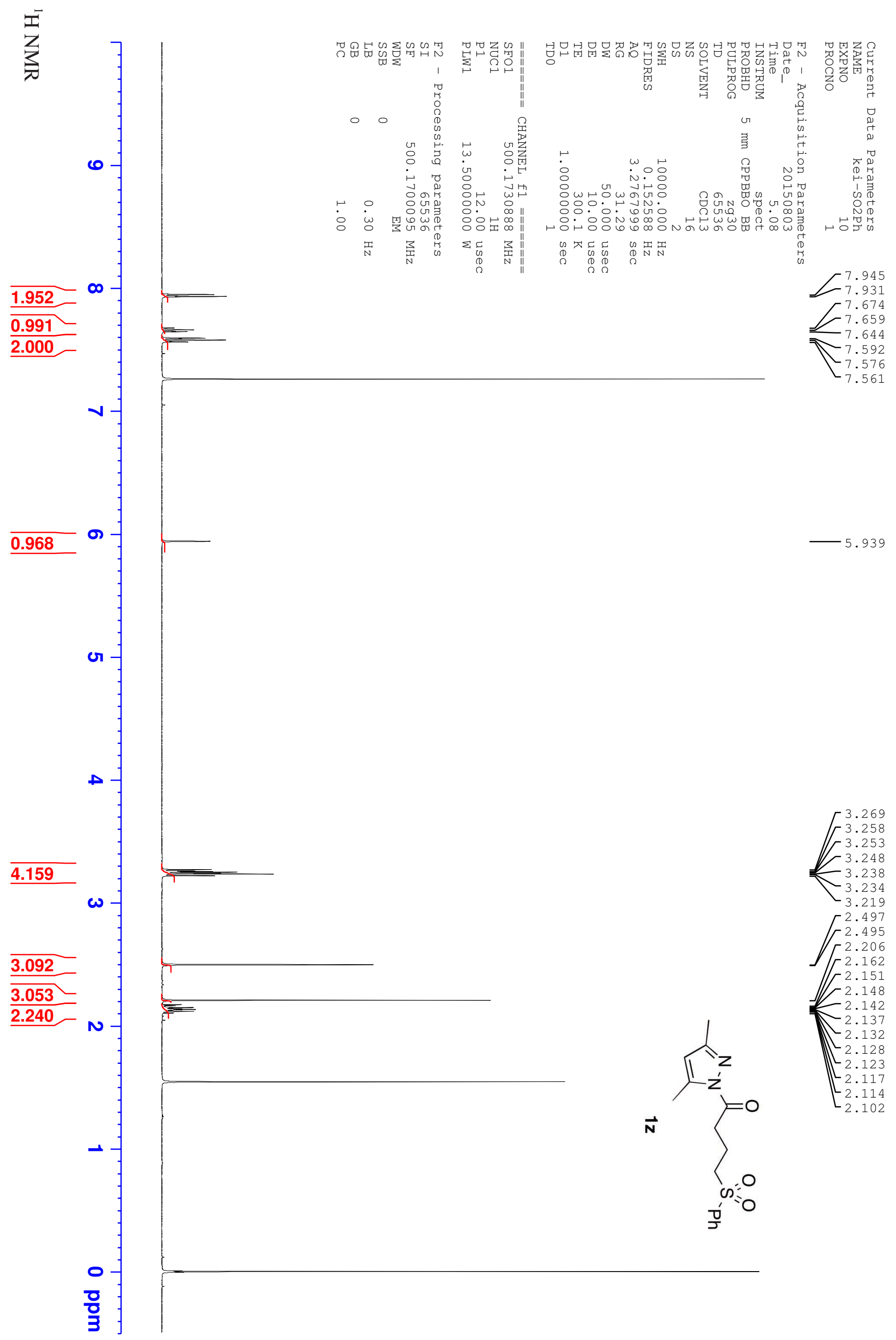


$\overbrace{}^{3}$

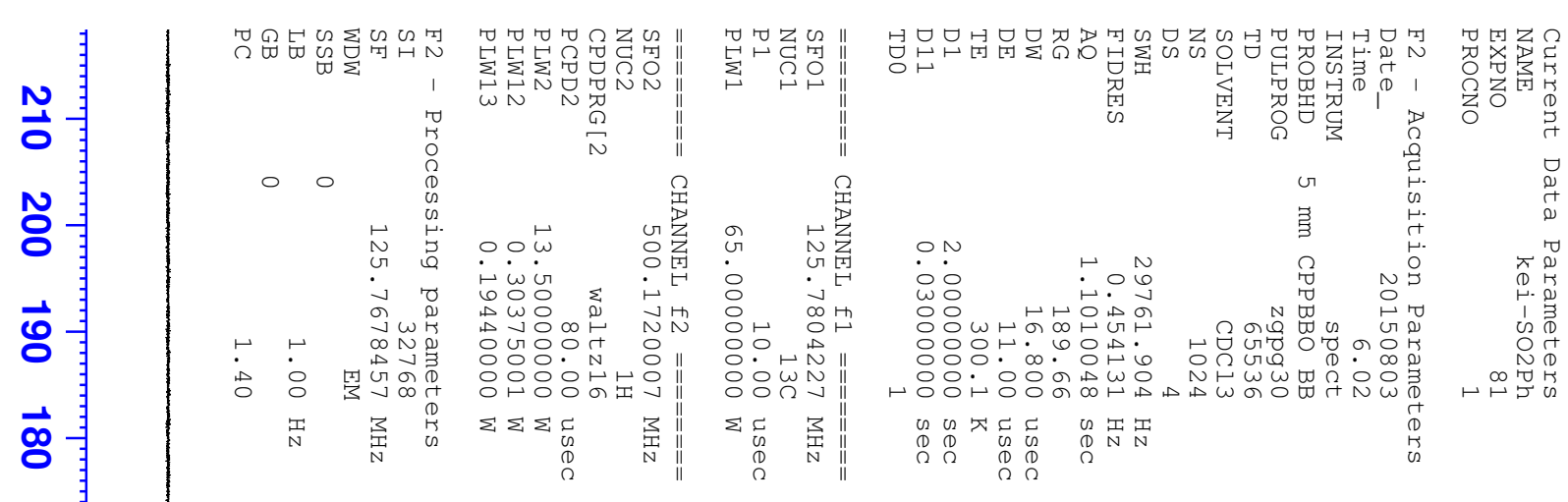

$\vec{\delta}$

$\overrightarrow{8}$

깅

$\overrightarrow{\mathrm{o}}$

$\vec{\omega}$

$\overrightarrow{\tilde{O}}$

$\overrightarrow{0}$

$\overrightarrow{8}$

8

ø

o

8

응

o

ఱ

ง
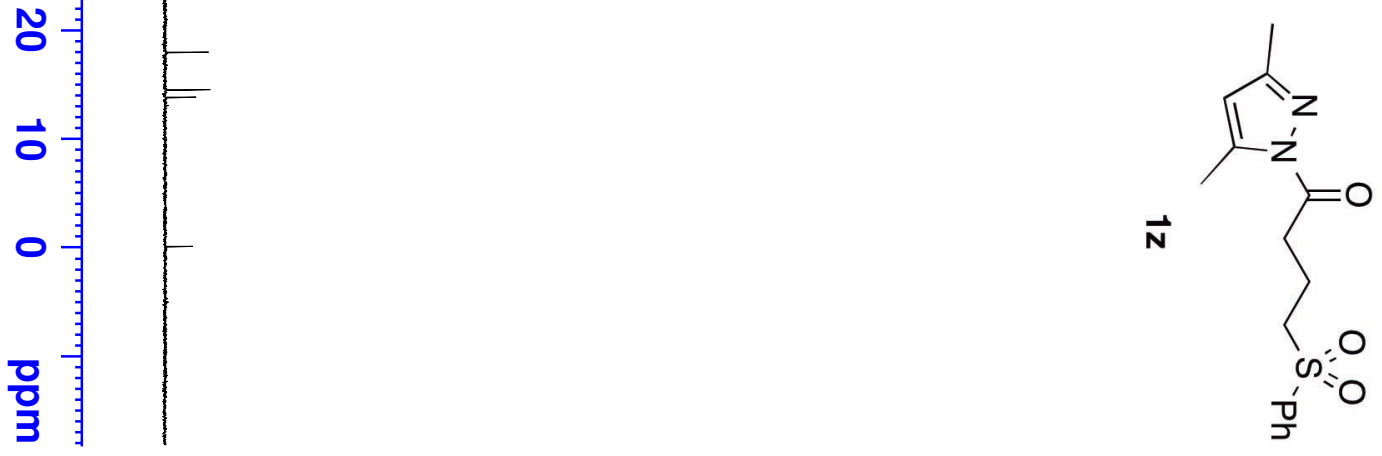

$-55.267$

$-33.441$

$-133.732$

-129.326
-128.150

$-111.294$

$-17.930$

$\sim 14.427$

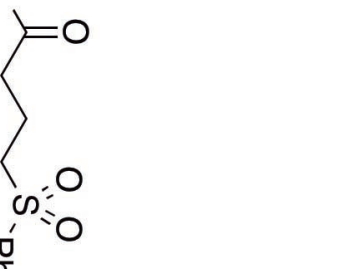




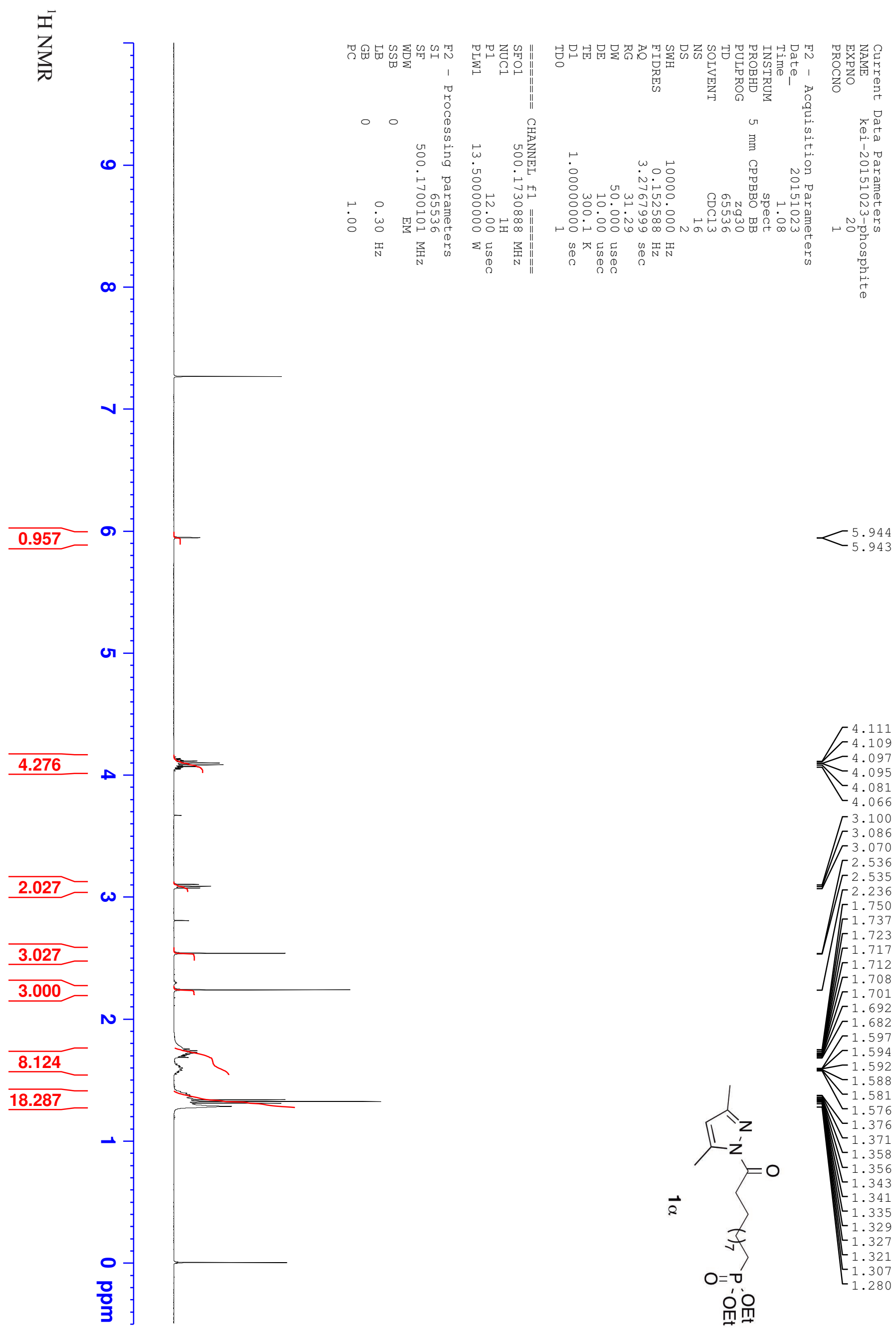


$\overbrace{}^{2}$
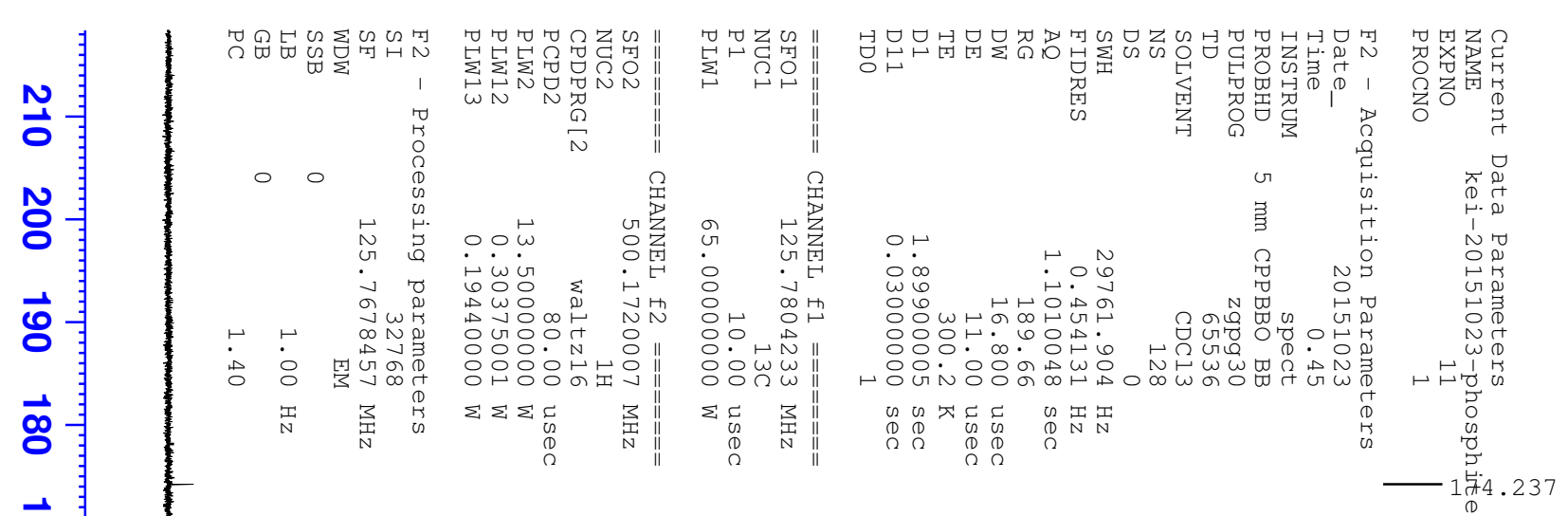

$-151.712$

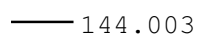

$-110.930$

$\overrightarrow{0}$

$\overrightarrow{\mathrm{s}}$

8

\&

ป

8

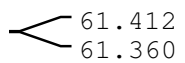

잉

b

w

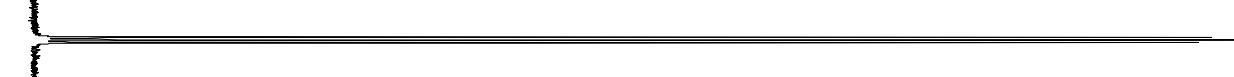

용

ก

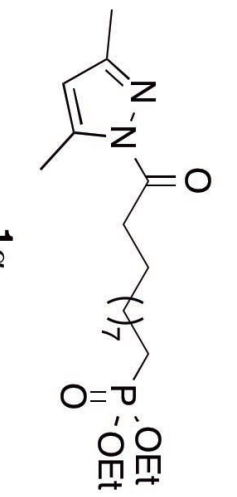

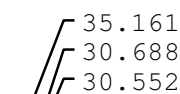

29.403
-29.352

29.352
29.121

$\begin{array}{r}29.121 \\ 29.091 \\ \hline\end{array}$

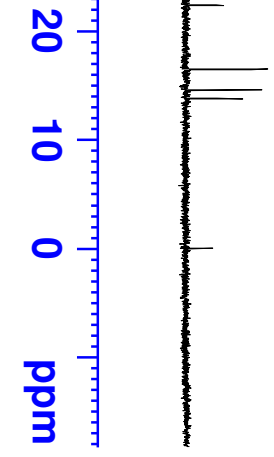

$\varlimsup^{26.27}$

25.156

24.301
-22.438

22.397

$-16.512$

$\left[\begin{array}{l}14.606 \\ 13.812\end{array}\right.$

13.812 


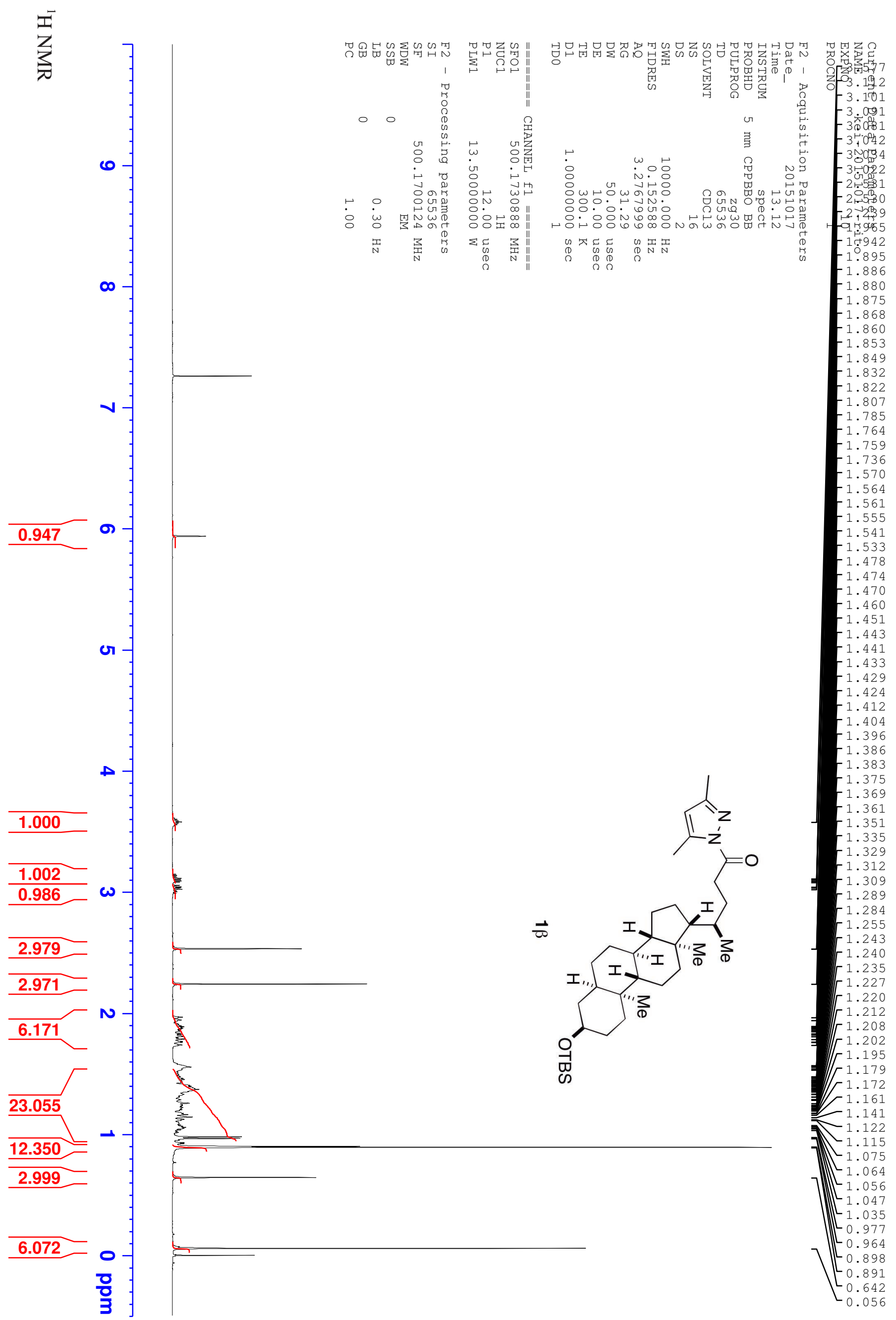


$\overbrace{0}^{\bar{\omega}}$

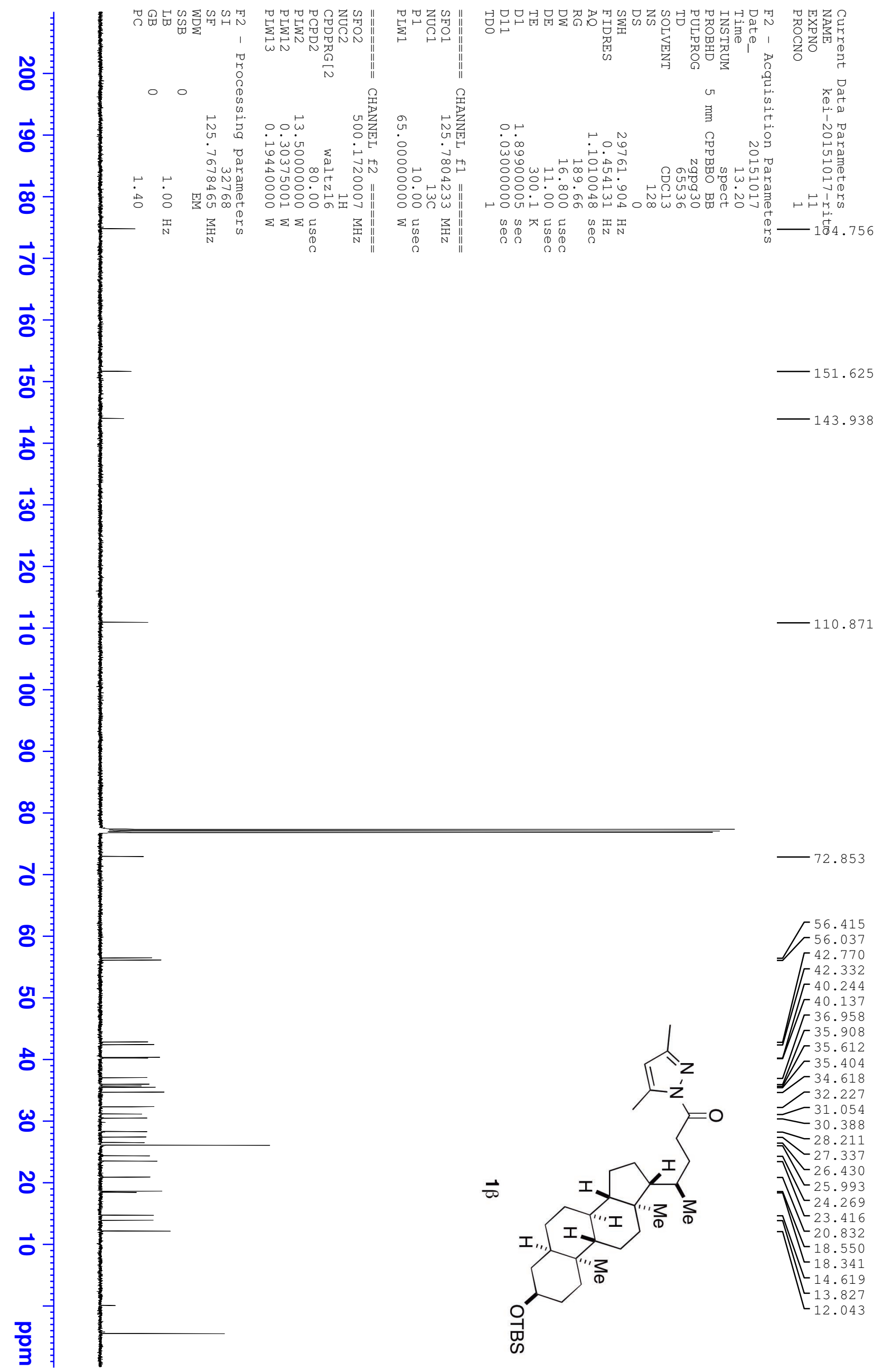




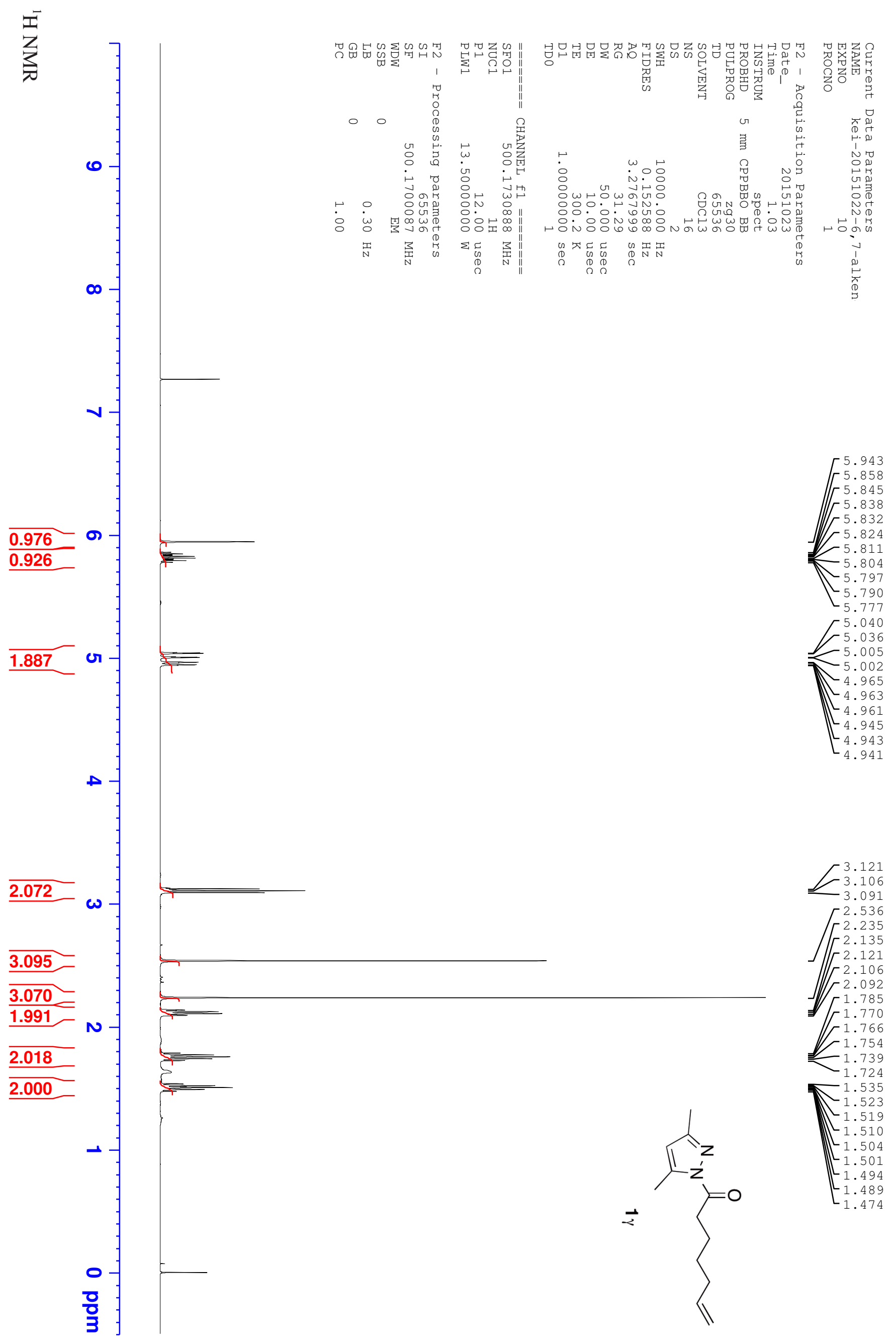


$\overbrace{0}^{\bar{\omega}}$

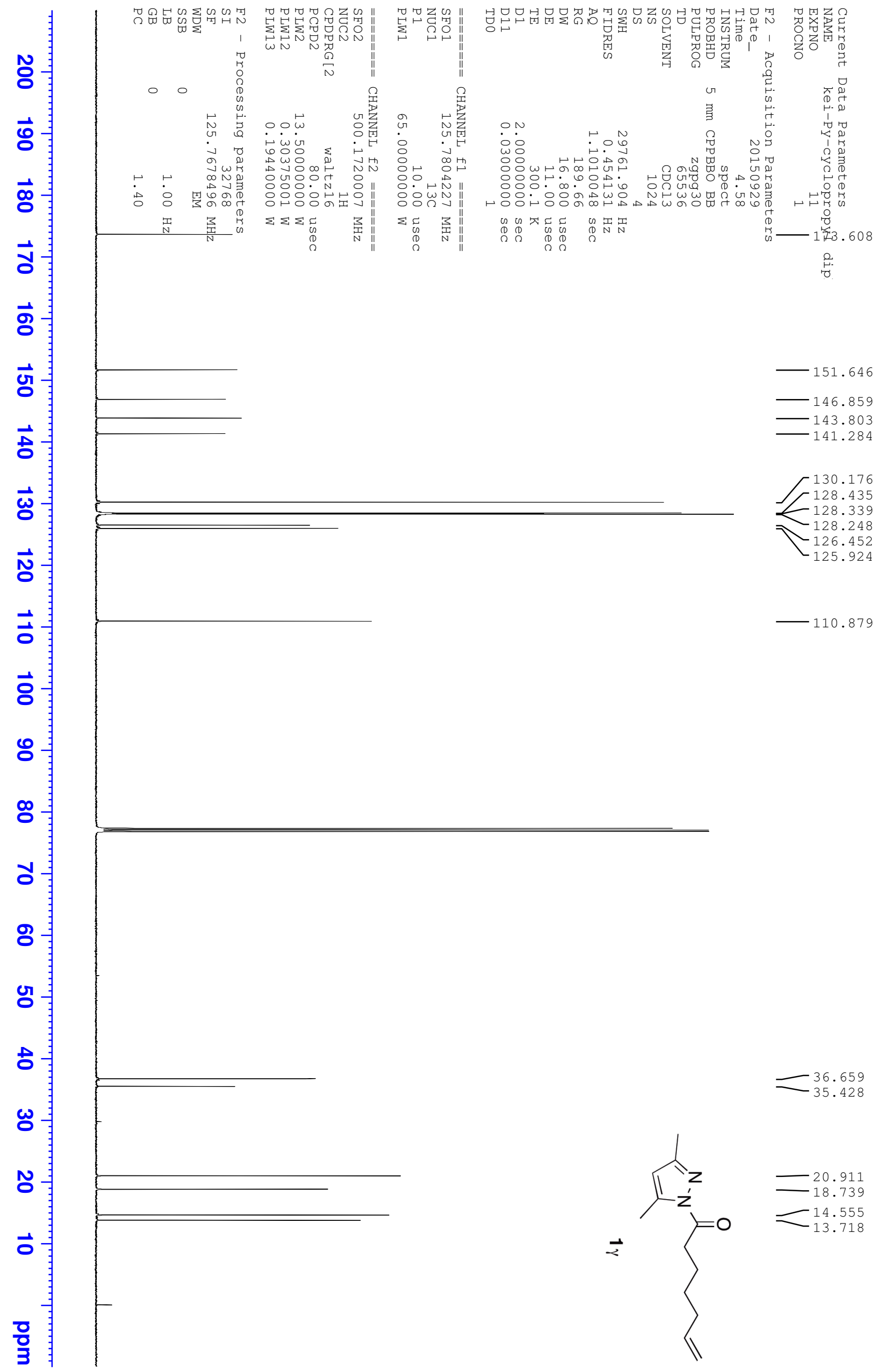




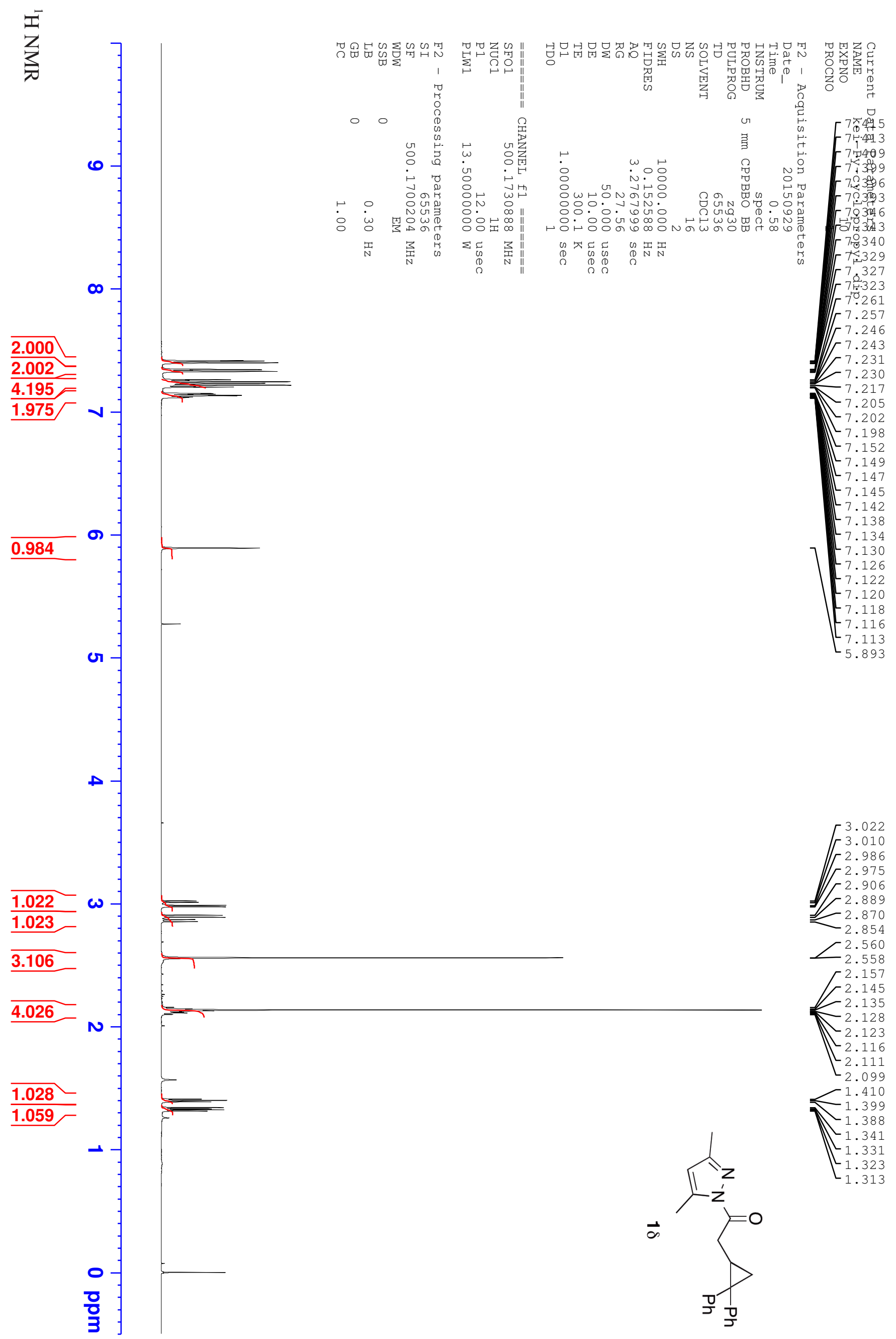


$\overbrace{0}^{\bar{\omega}}$

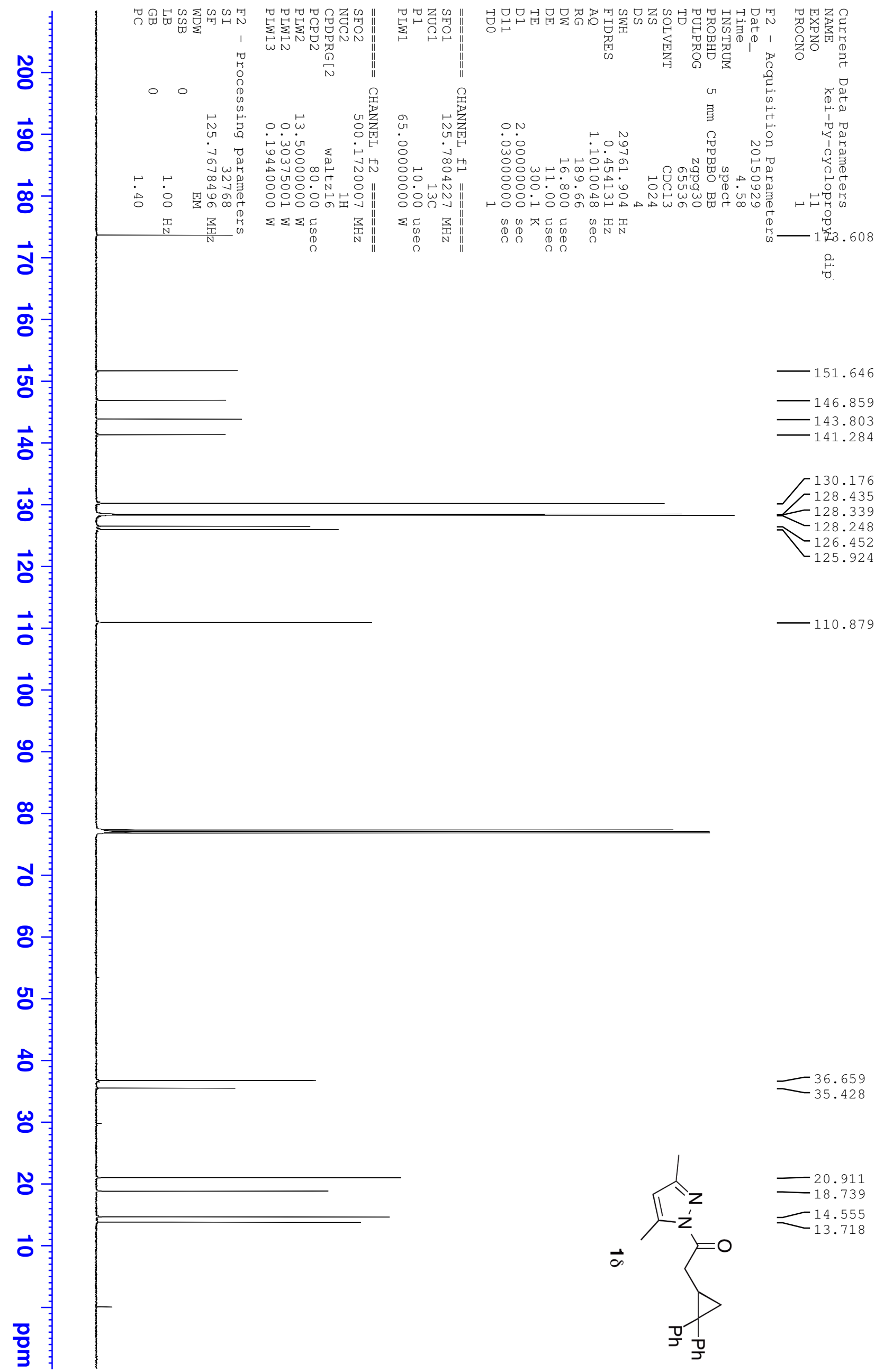




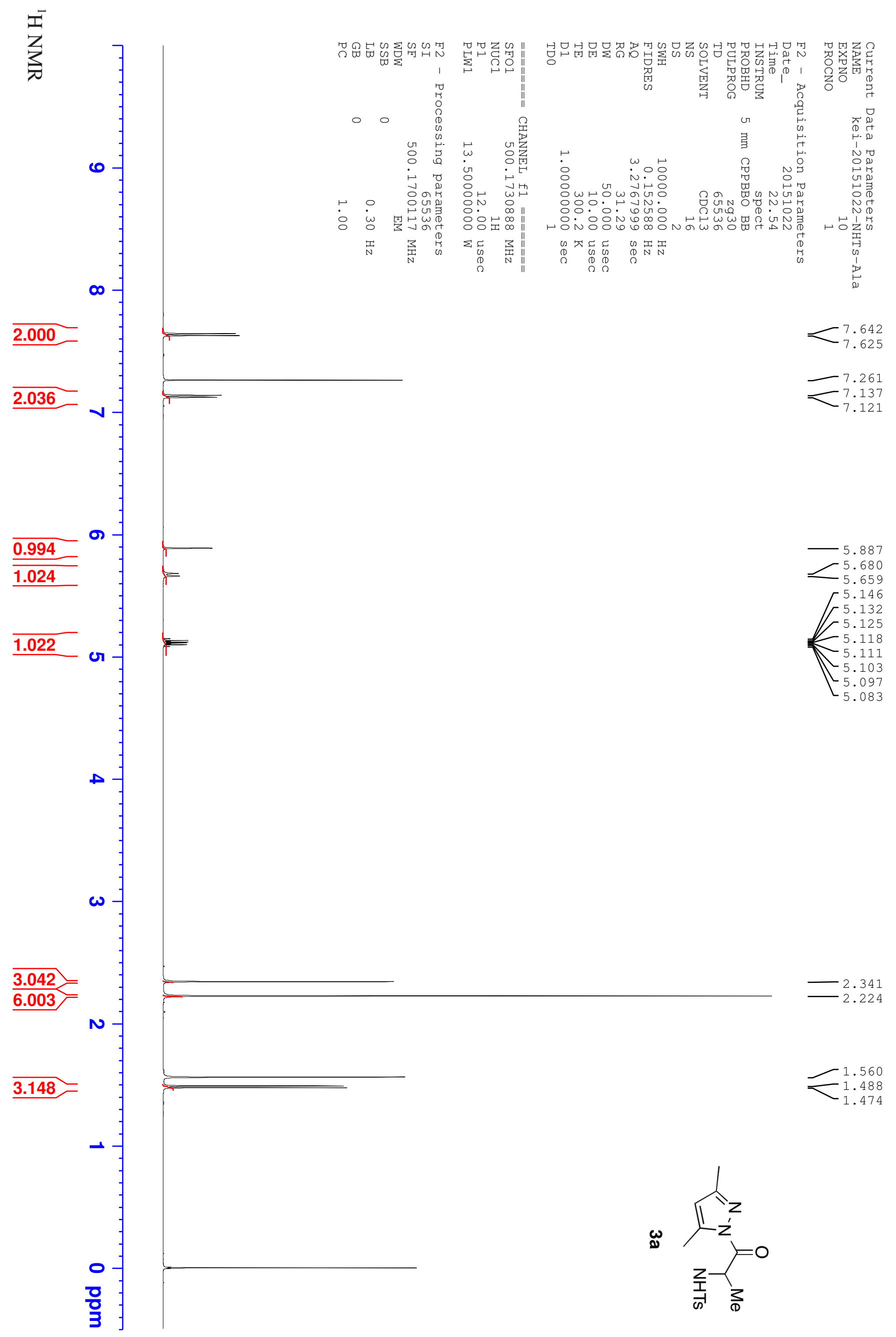


$\overbrace{}^{\bar{\omega}}$

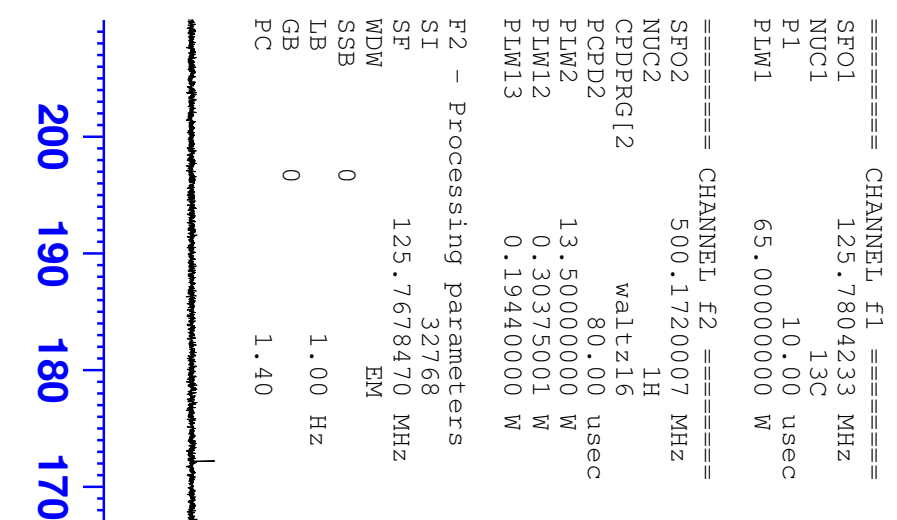

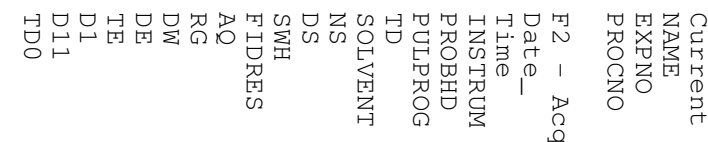

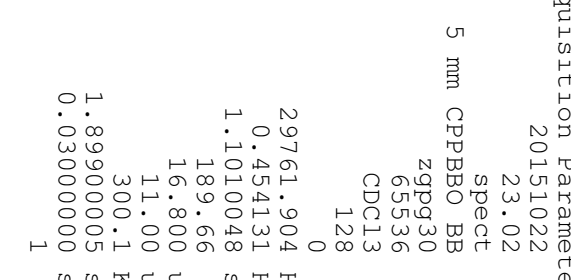

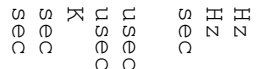

$\vec{\circ}$

ज्ञ

$\vec{p}$

$-144.387$

143.352

$-136.621$

$\vec{\omega}$

$-129.311$

127.252

$\overrightarrow{\text { O }}$

$\vec{\partial}$

$\overrightarrow{\mathrm{s}}$

8

๓

o

8

용

8

$\omega$

ก

唪

$\overrightarrow{0}$

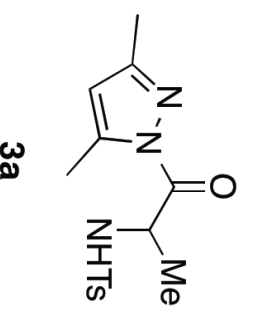

$-111.403$

$-77.265$

77.012
-76.758

$-52.357$

$=21.390$

20.273

$+13.830$

13.812

합 


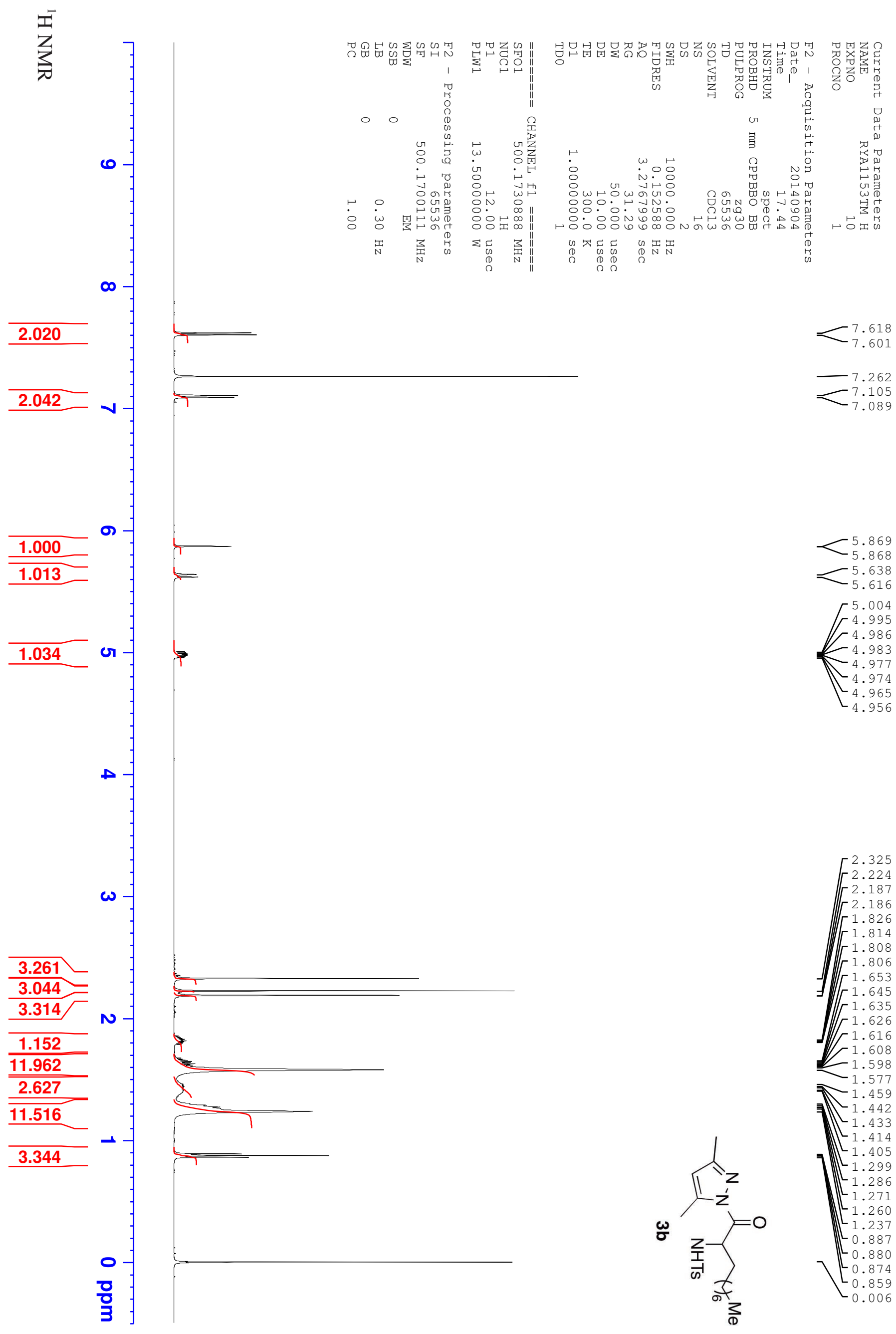


$\overbrace{0}^{\bar{\omega}}$

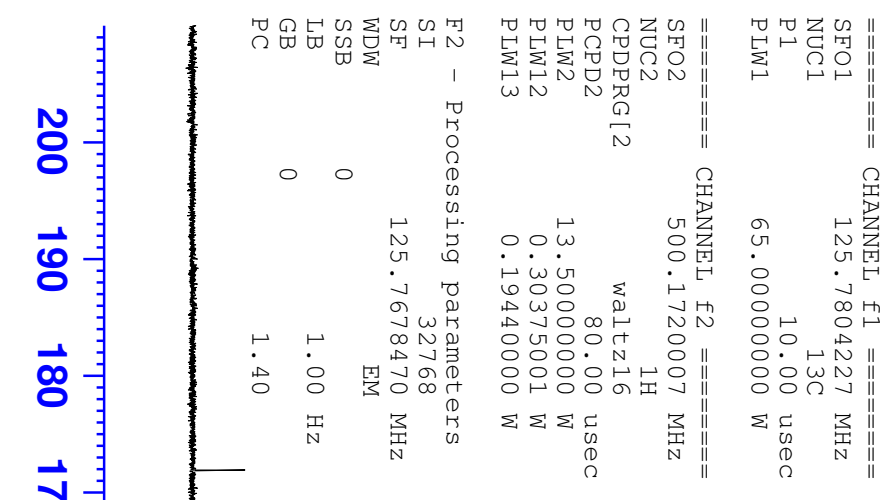

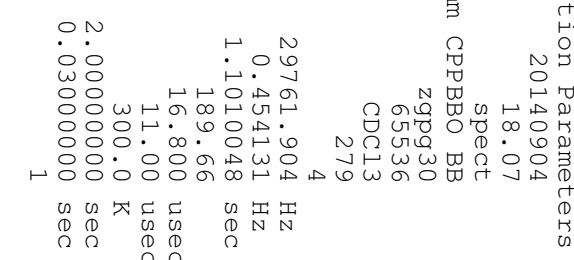

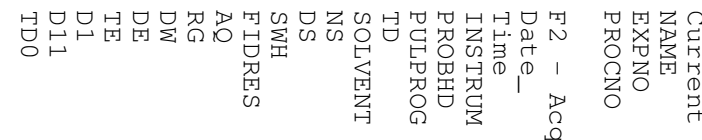

8

잉

o

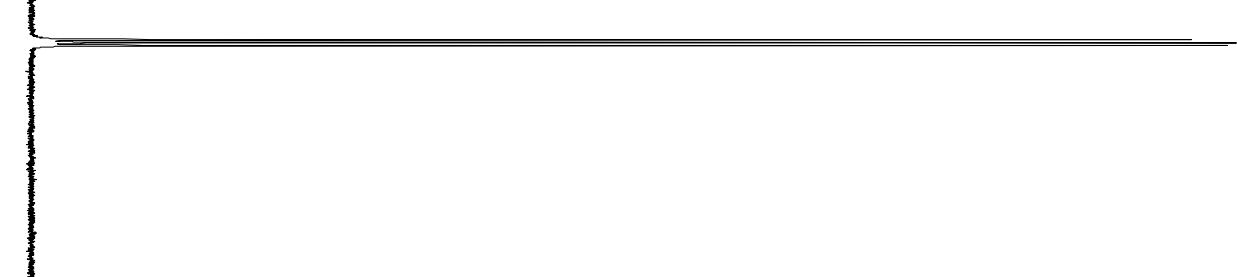




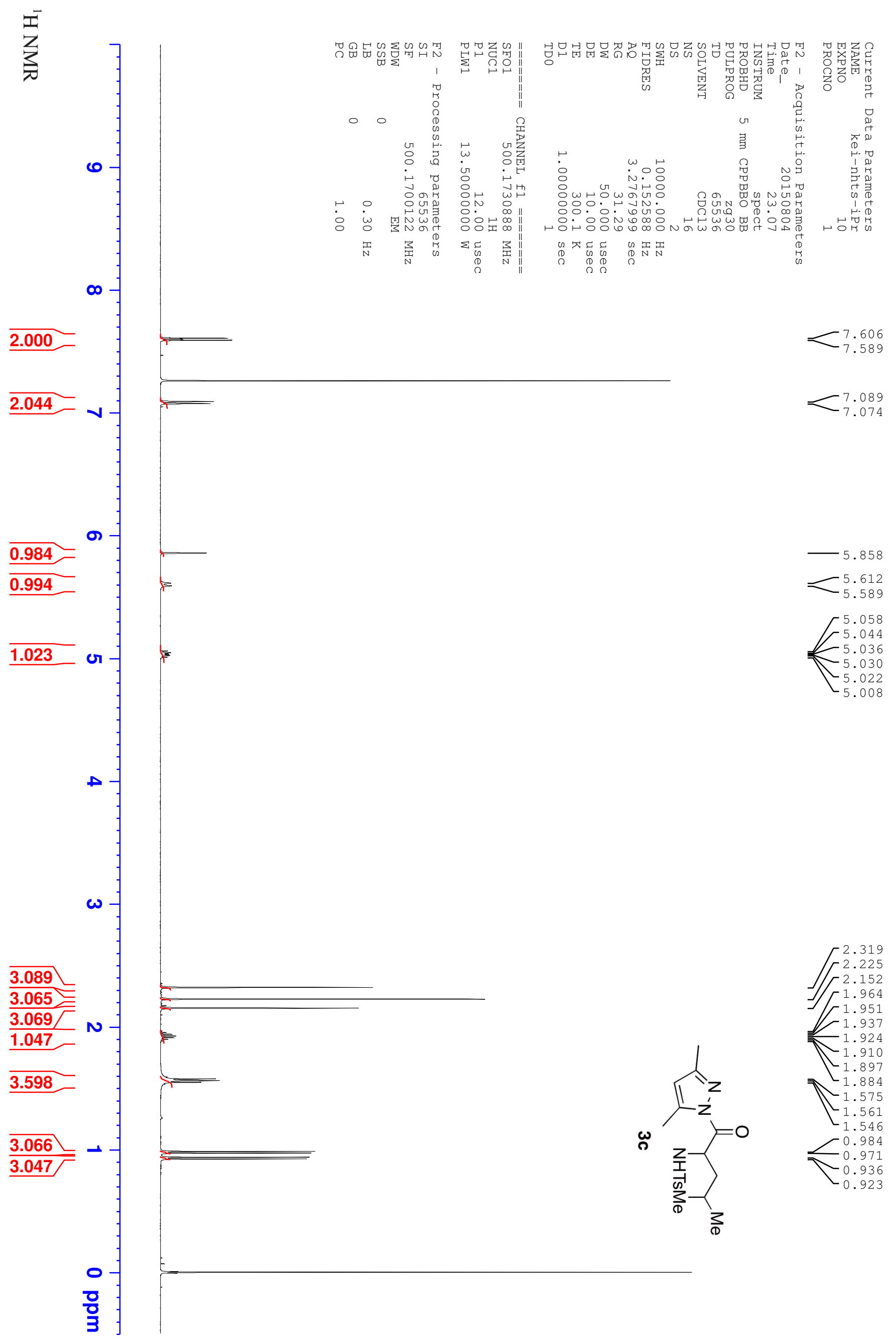


$\overbrace{}^{\bar{\omega}}$

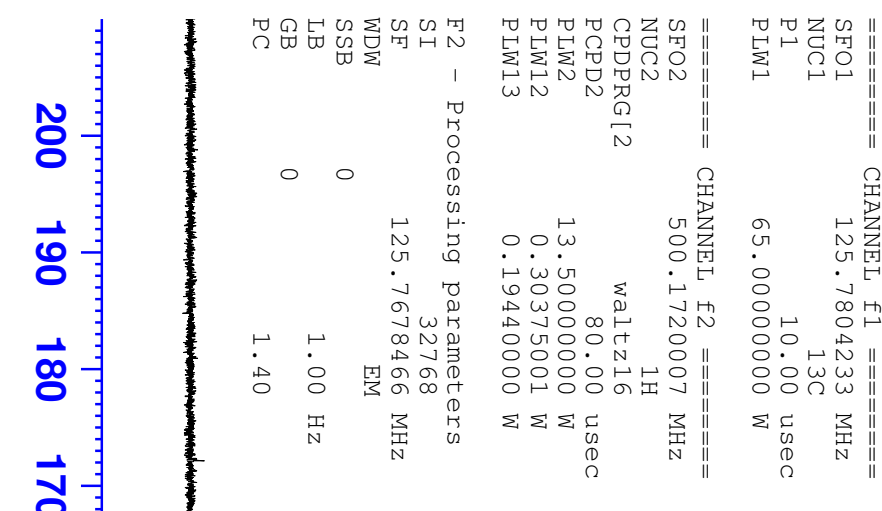

\section{,}

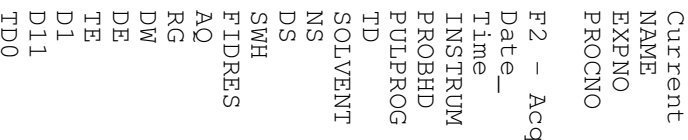

$\begin{array}{ll} & \\ & 3\end{array}$

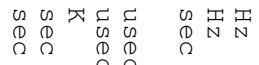

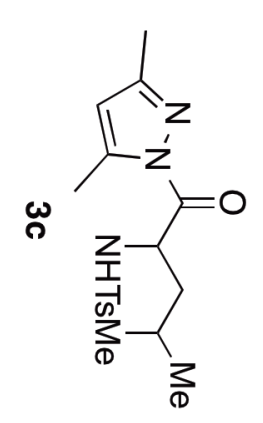

24.703
-23.136

24.703
23.1362

$-21.352$

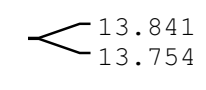




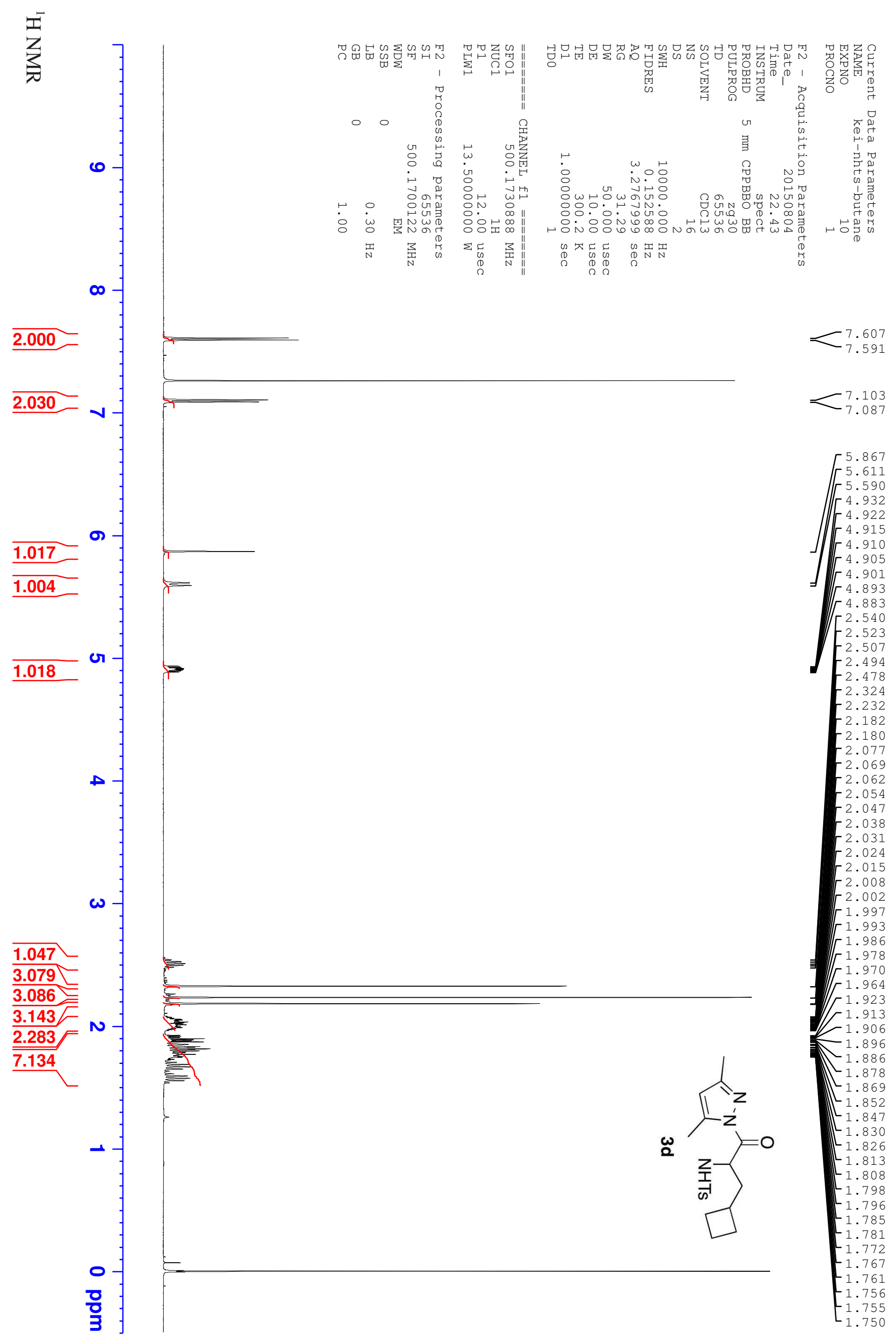


$\overbrace{}^{2}$

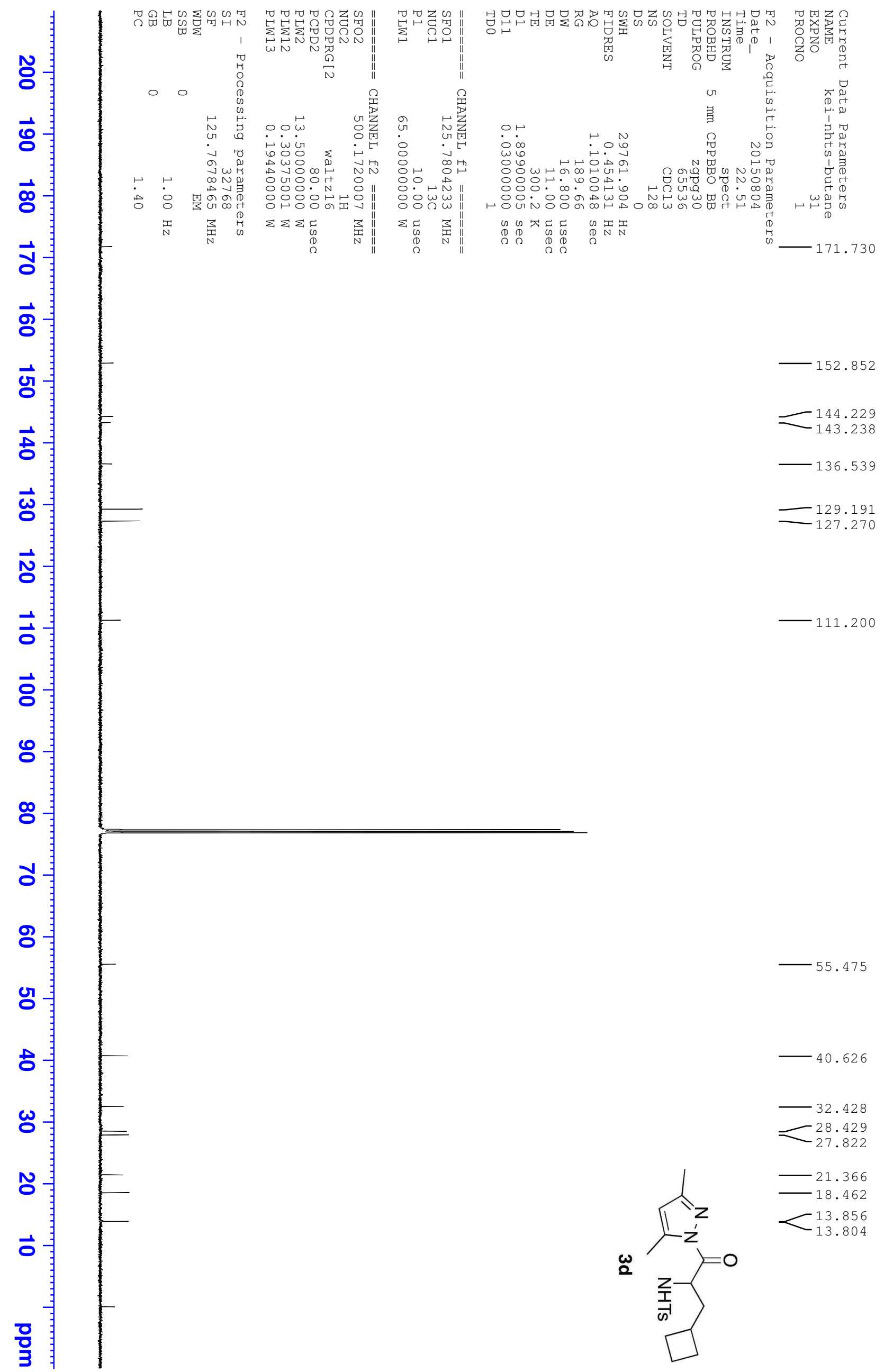




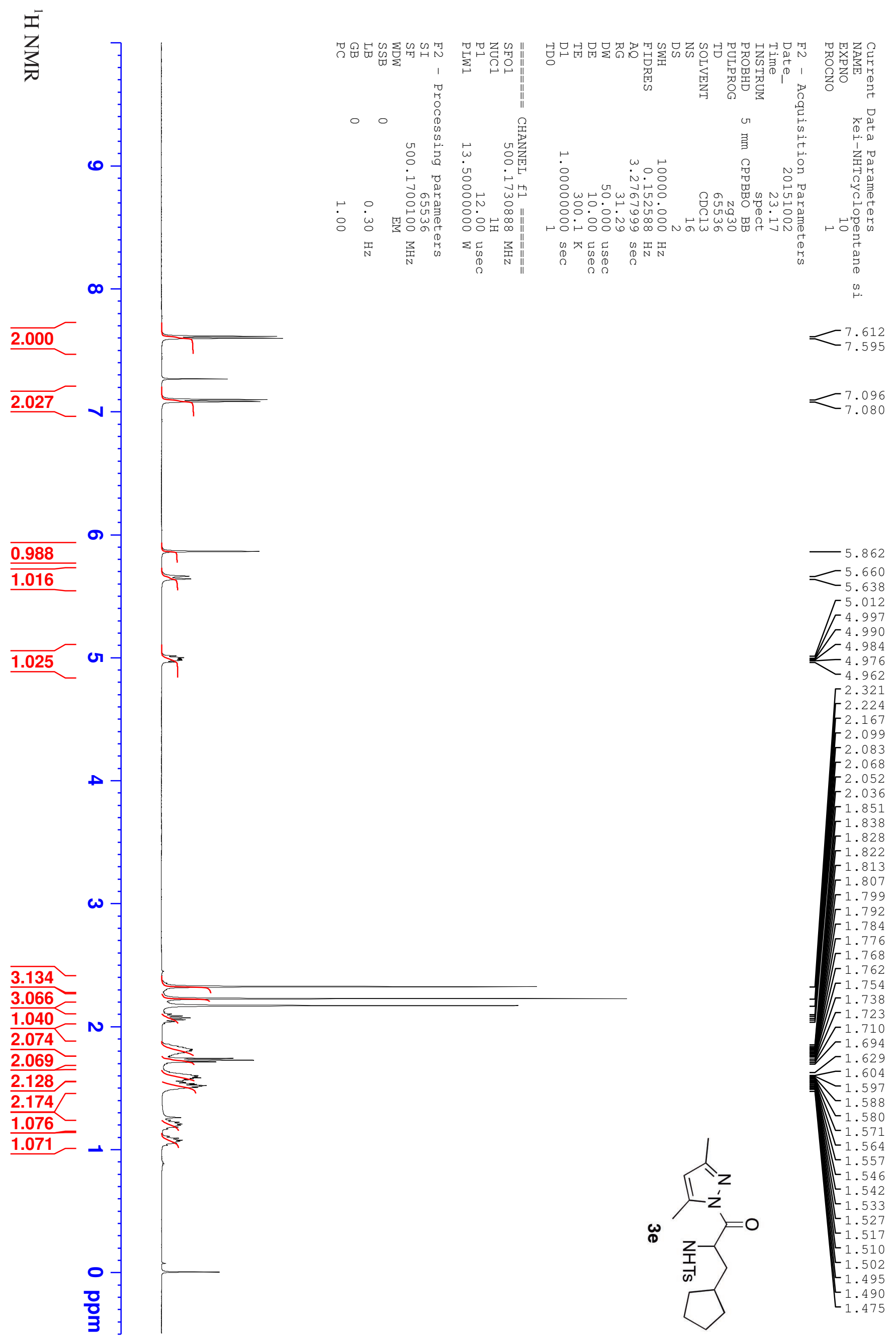


$\overbrace{}^{3}$

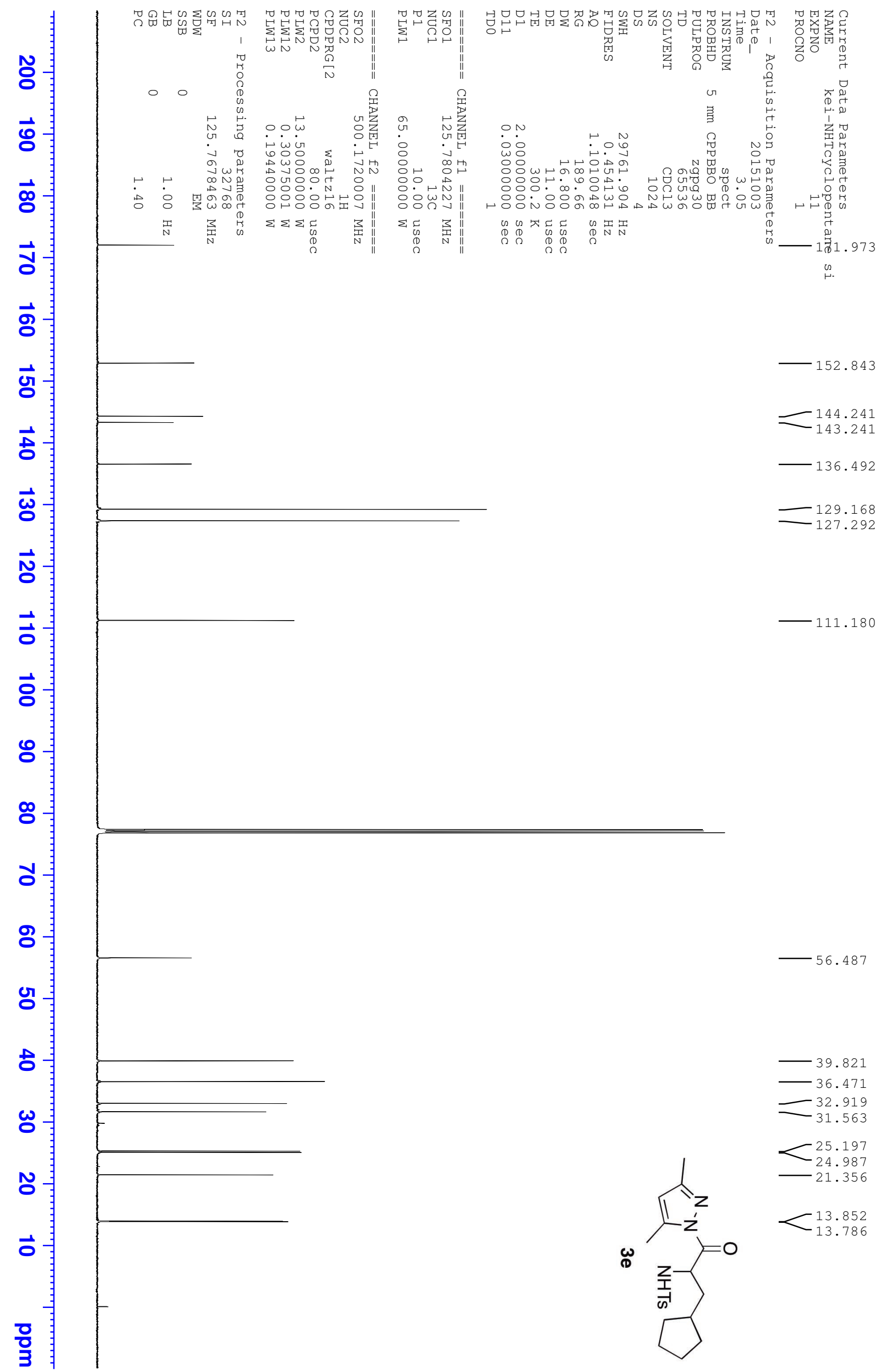




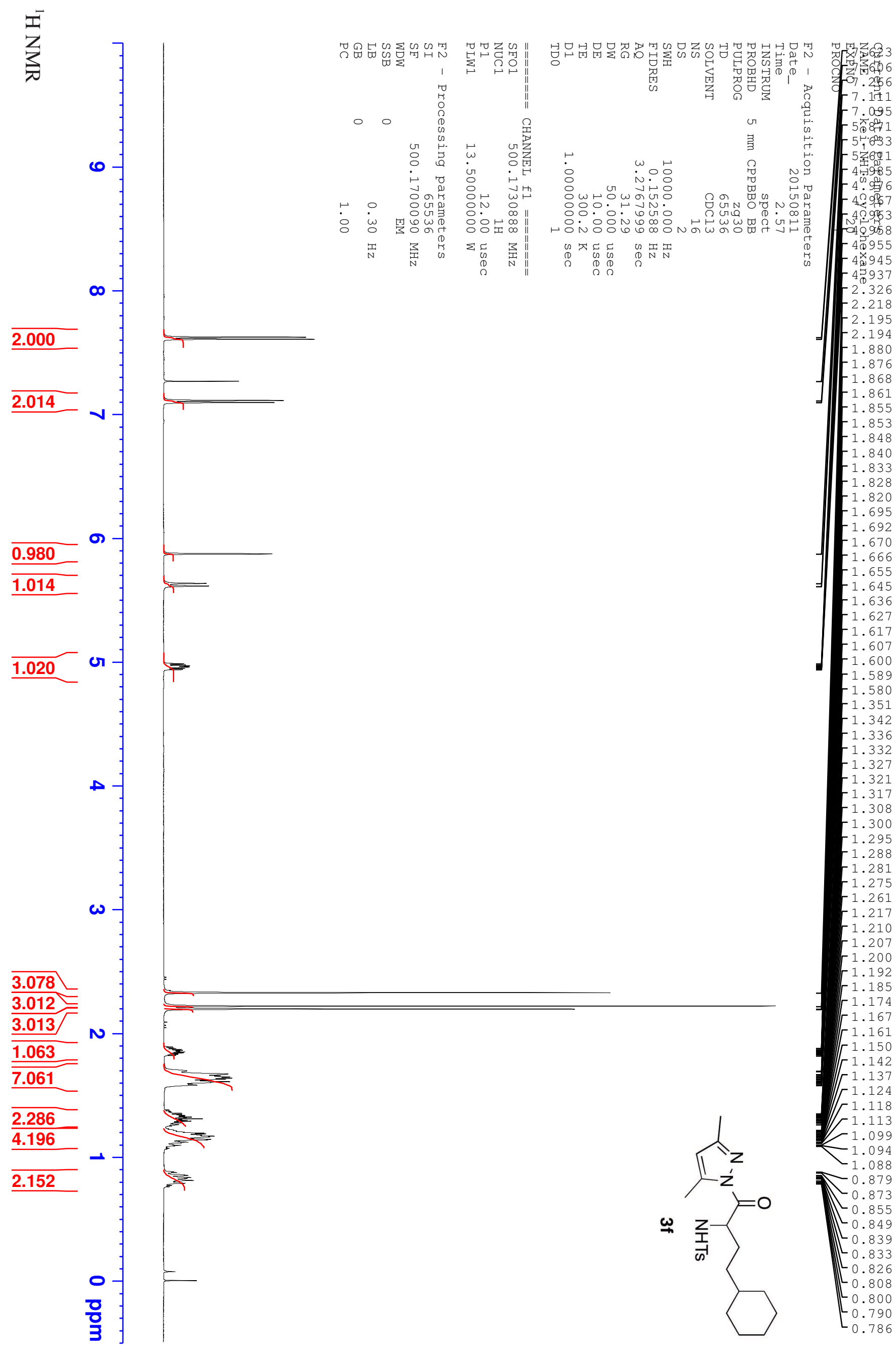


$\overbrace{}^{3}$

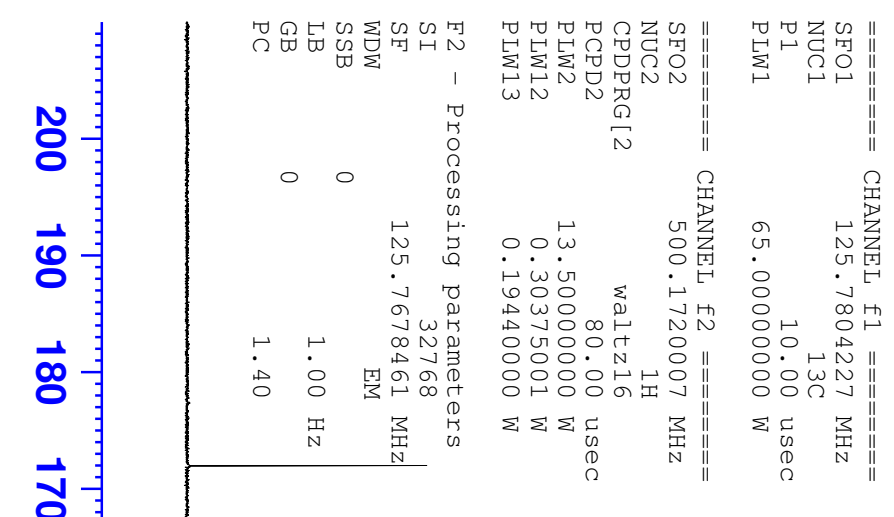

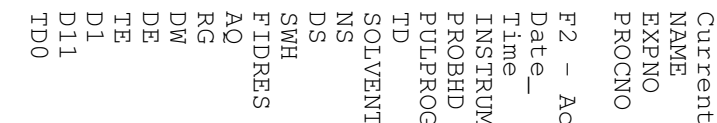

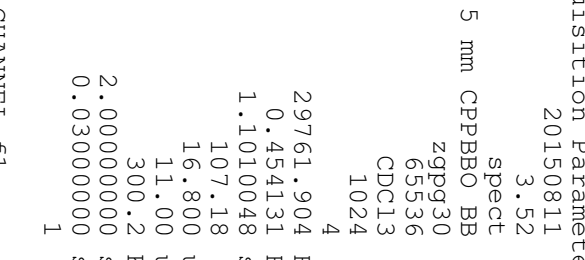

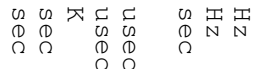
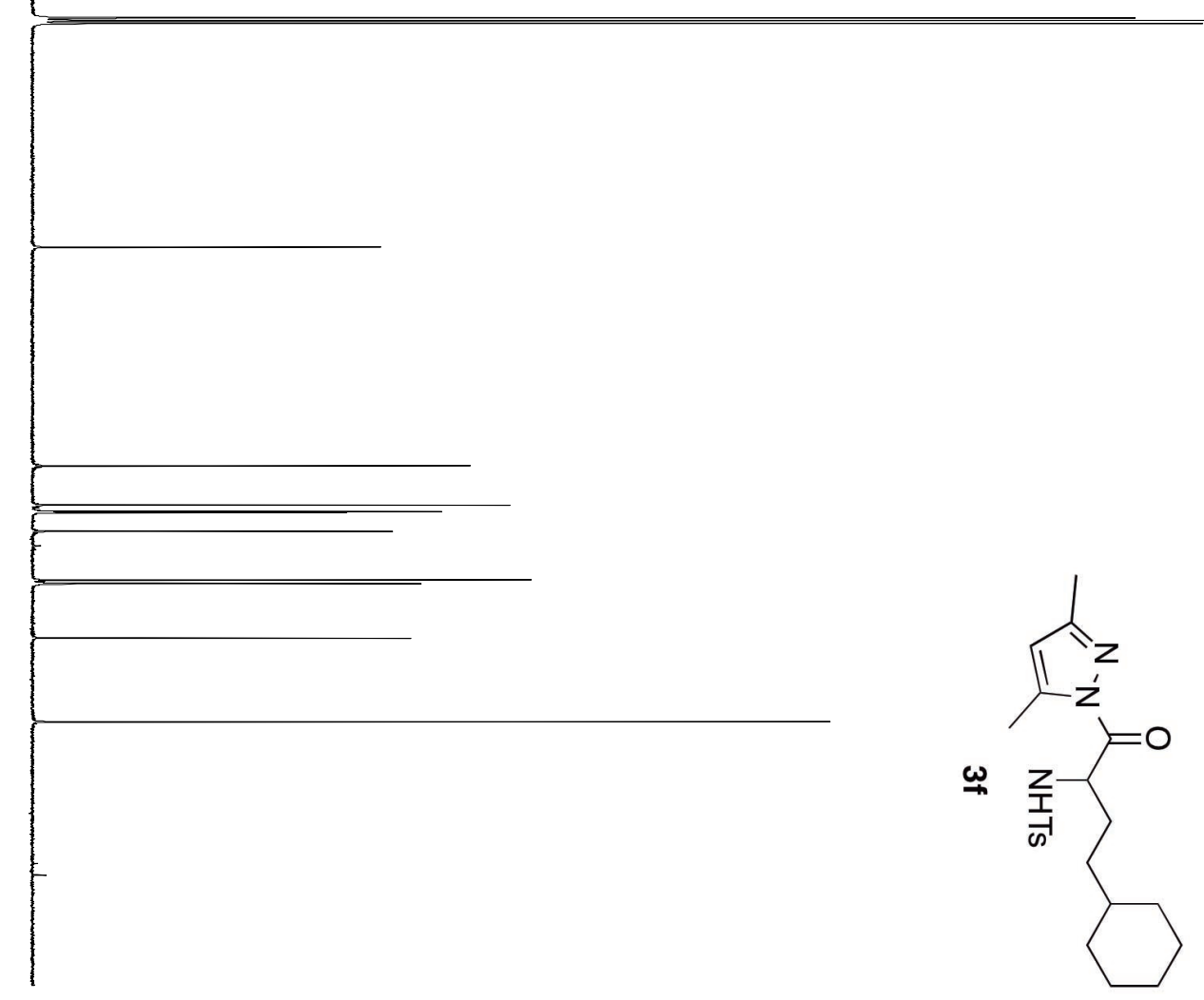

- 36.885 33.346 32.784 32.687
30.981 30.981
-26.594

26.594

26.249

$-21.364$ 


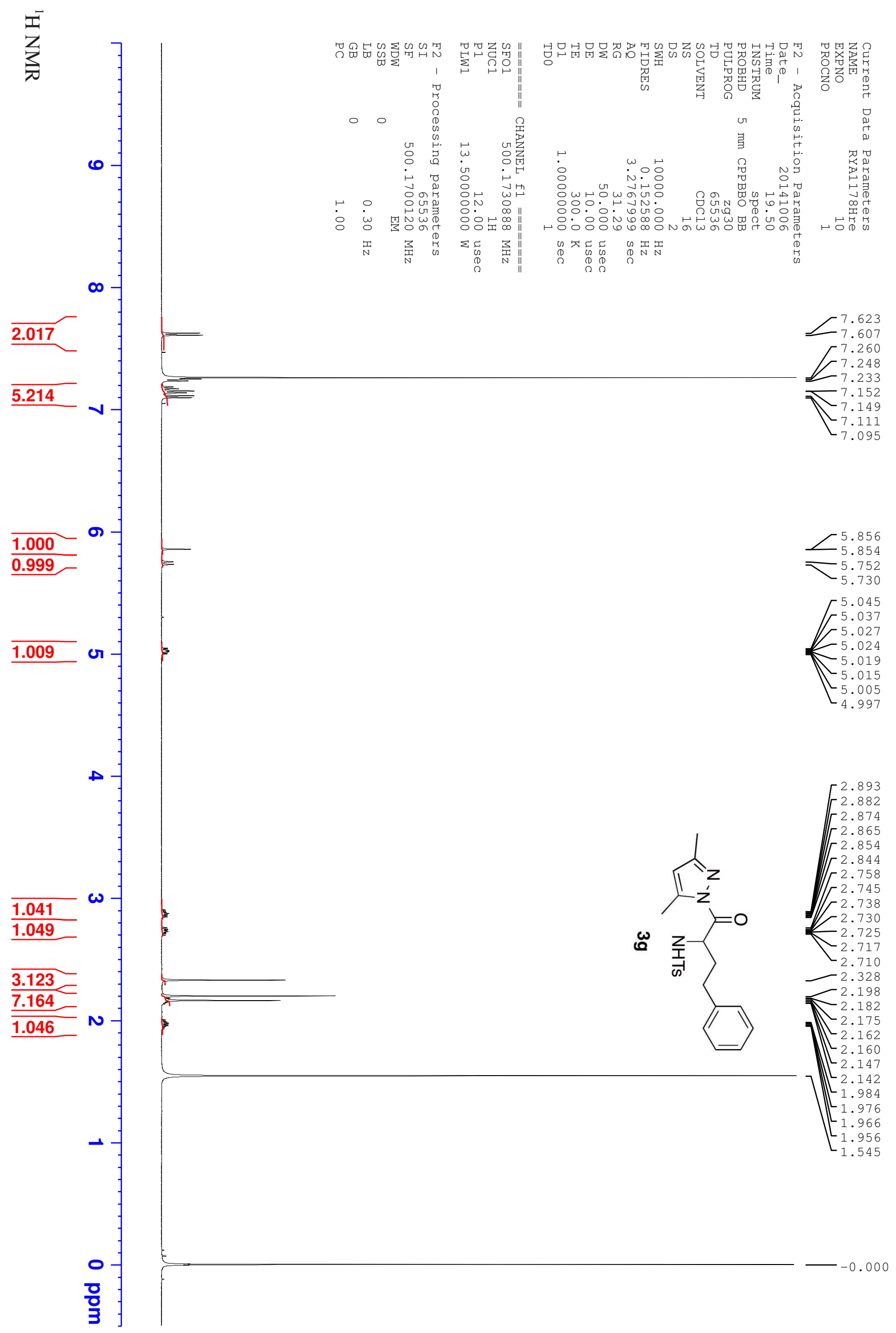


$\overbrace{}^{\bar{\omega}}$

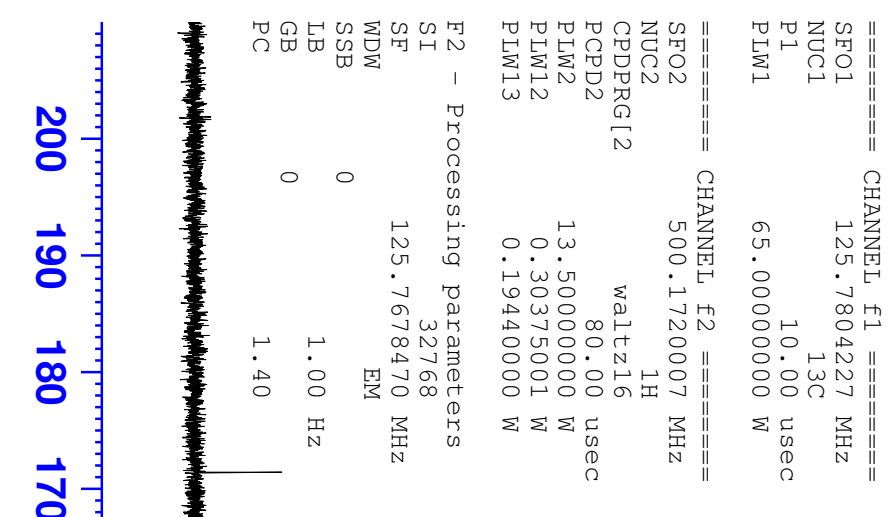

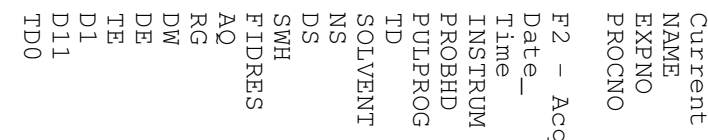

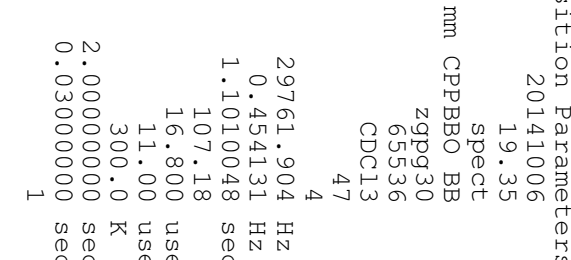

1.382

$\vec{\circ}$

ज्ञ

苦

$\simeq 144.294$

$-143.376$

$-136.445$

$\overrightarrow{\mathrm{\omega}}$

సัญ

$\vec{\Delta}$

$\overrightarrow{\mathrm{s}}$

8

๓

๖

8

g

to

ผ

กั

영

$\overrightarrow{0}$

흉

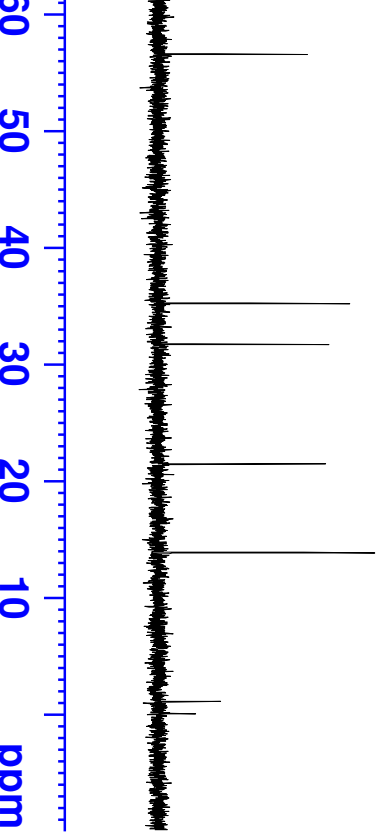

129.270

$-128.497$

$-127.298$

126.063

$-111.325$

$+77.273$

76.765

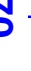




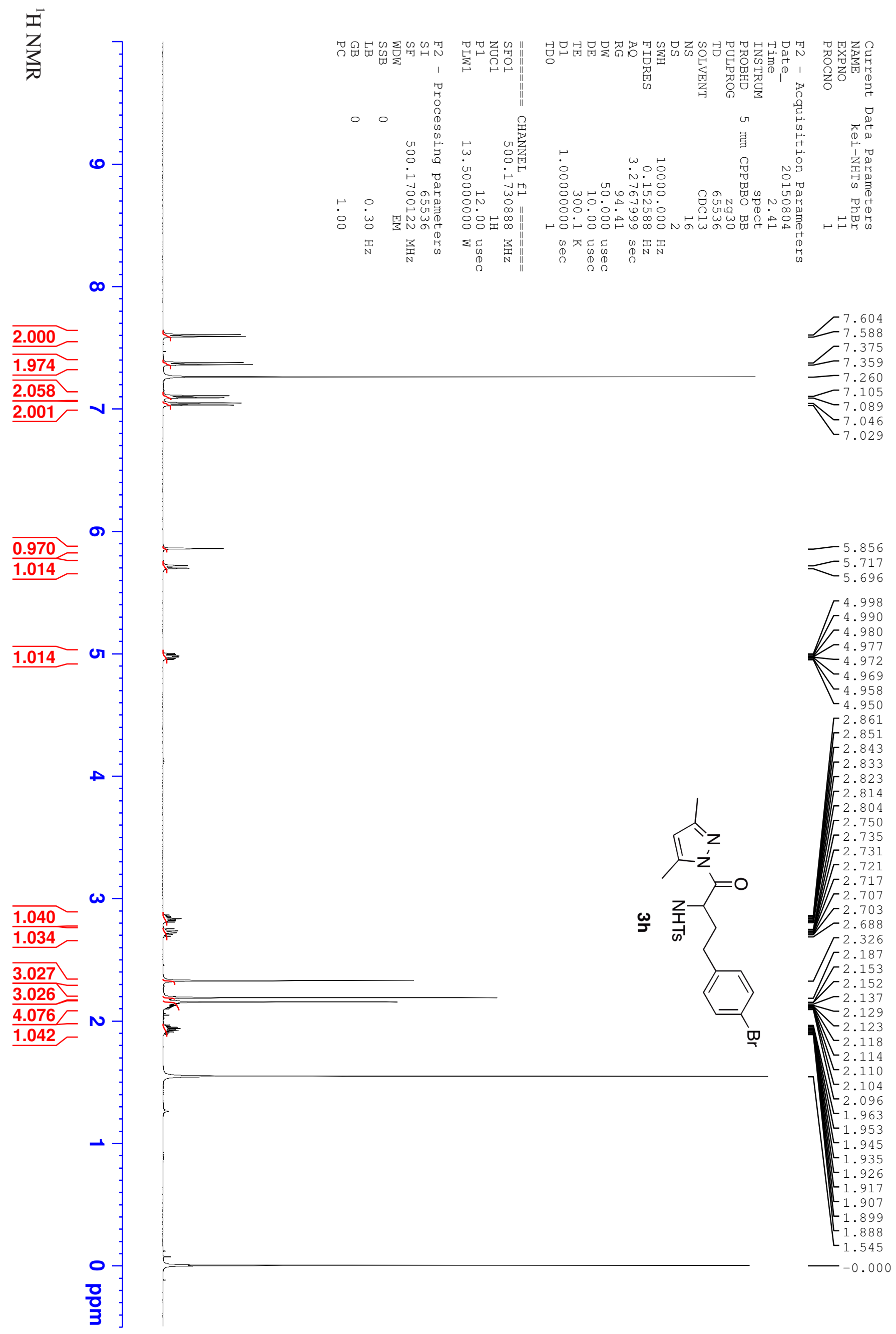


$\overbrace{}^{2}$

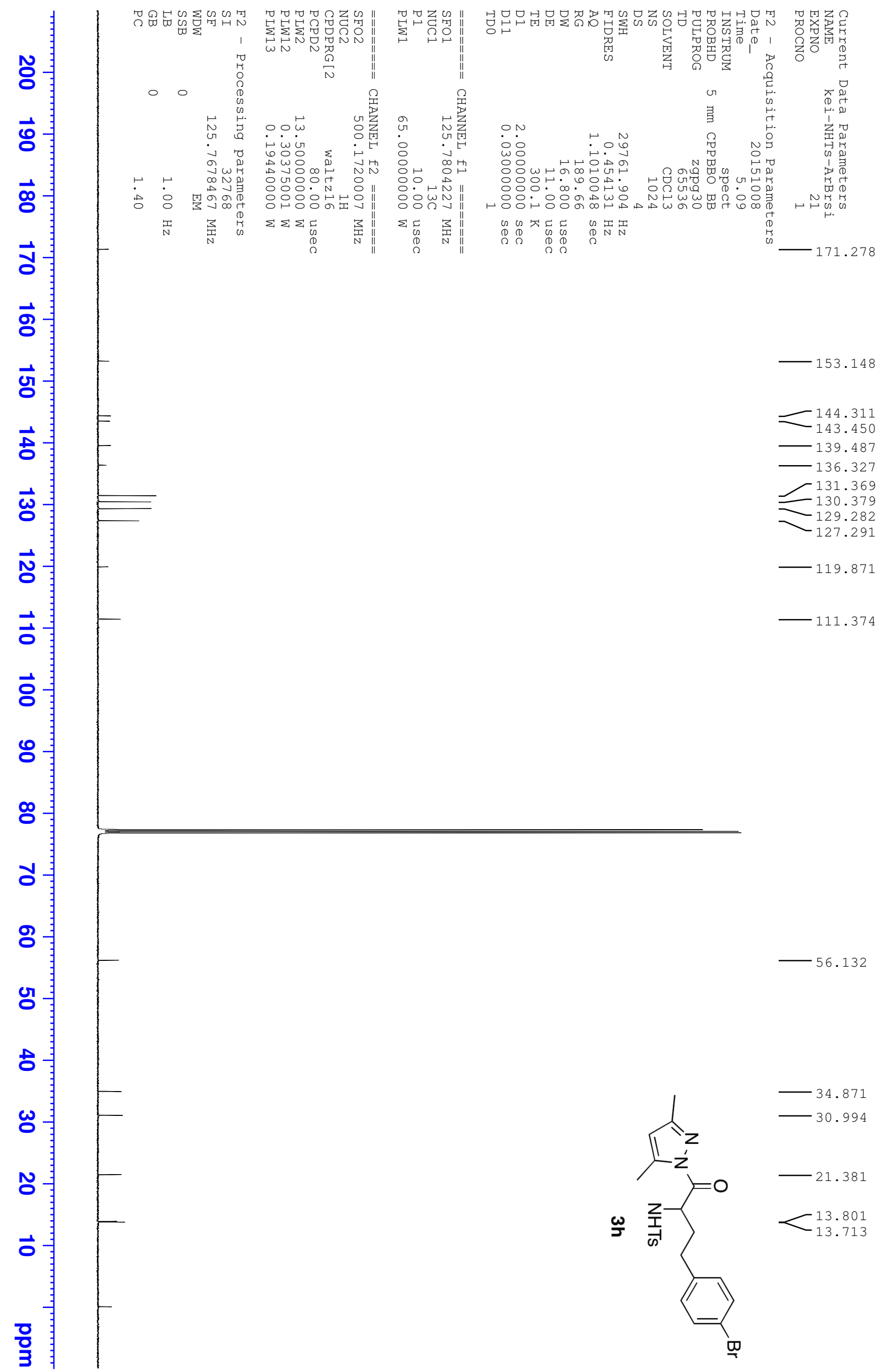




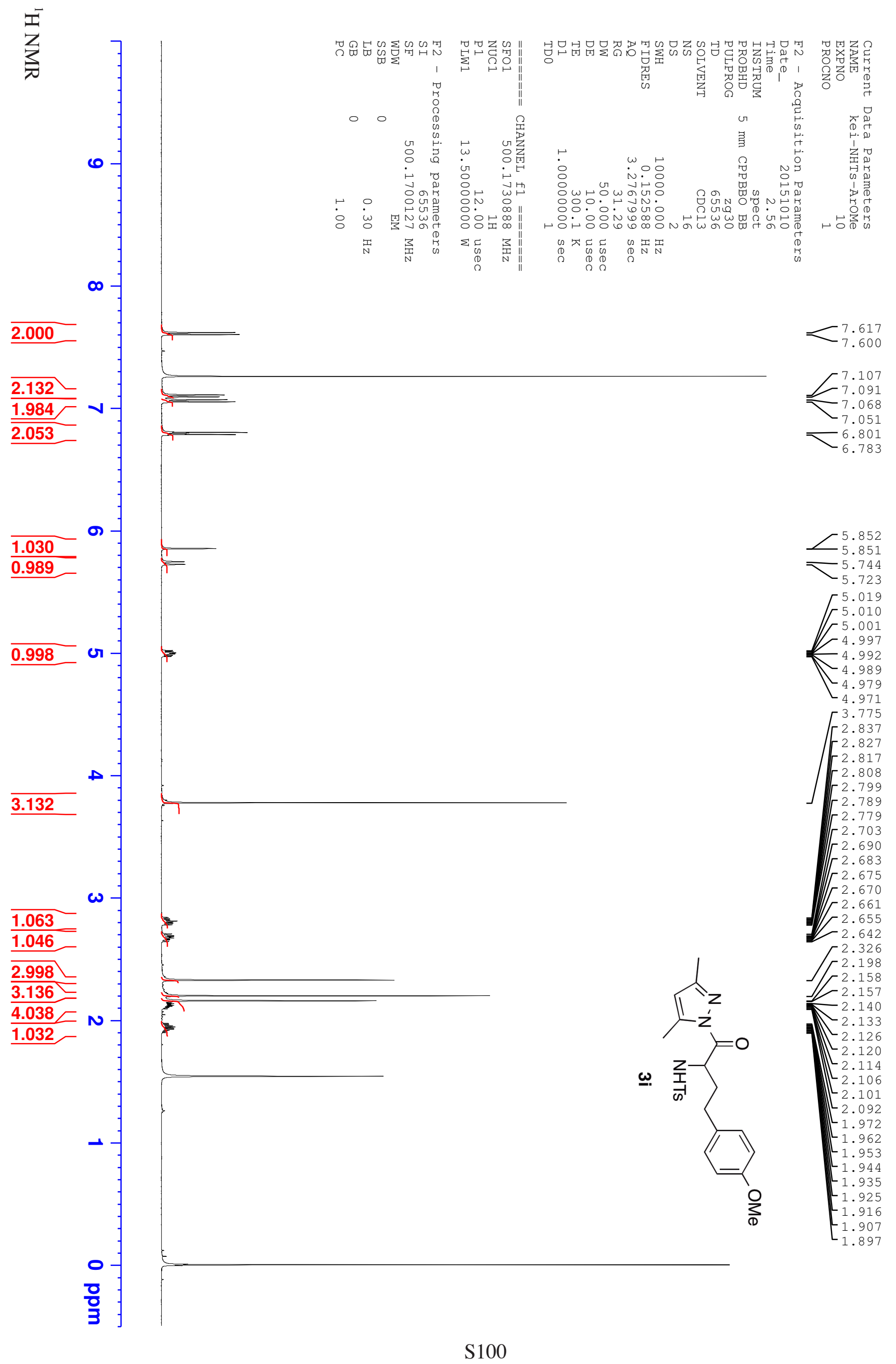


$\overbrace{}^{\bar{\omega}}$

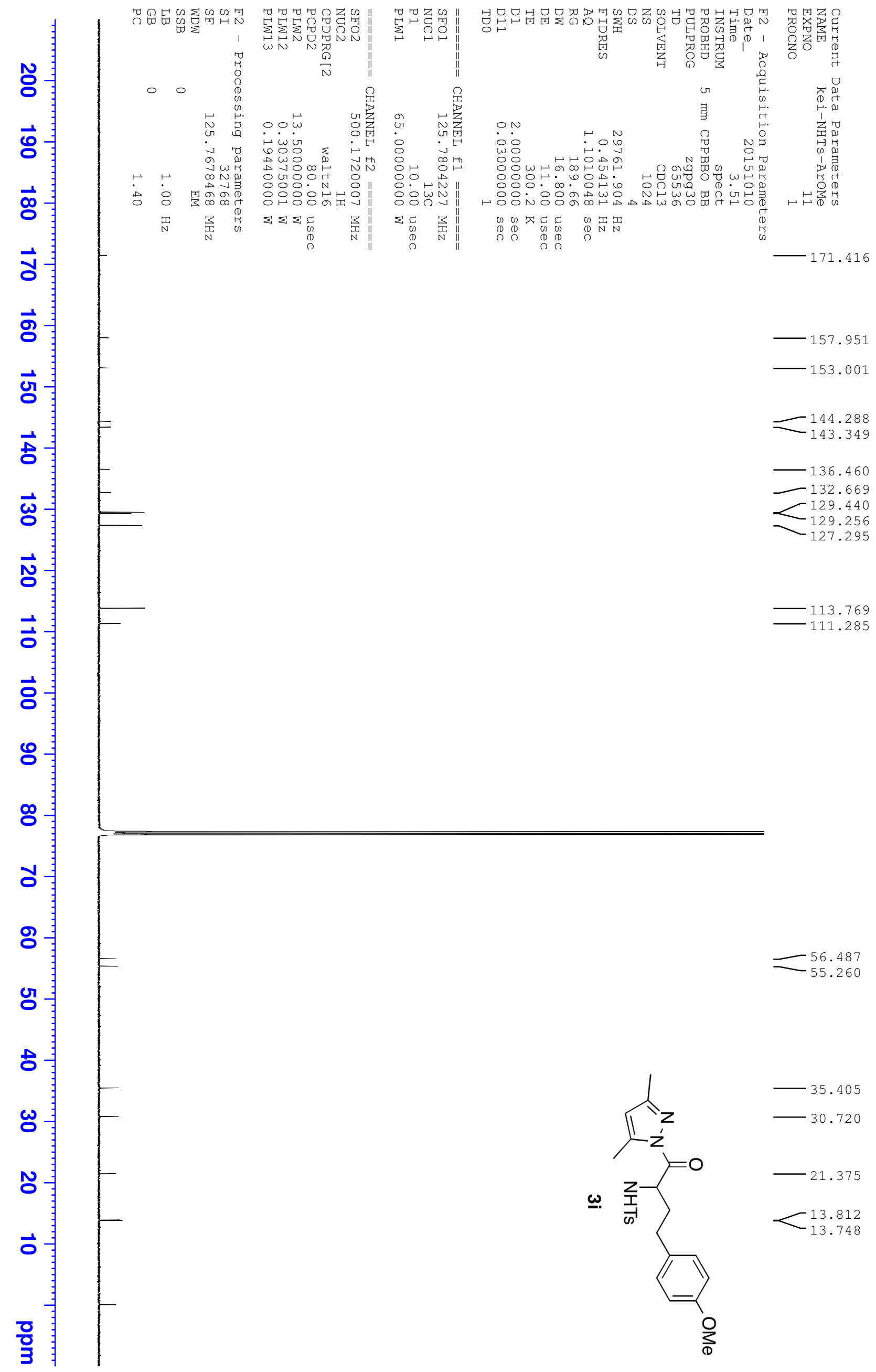




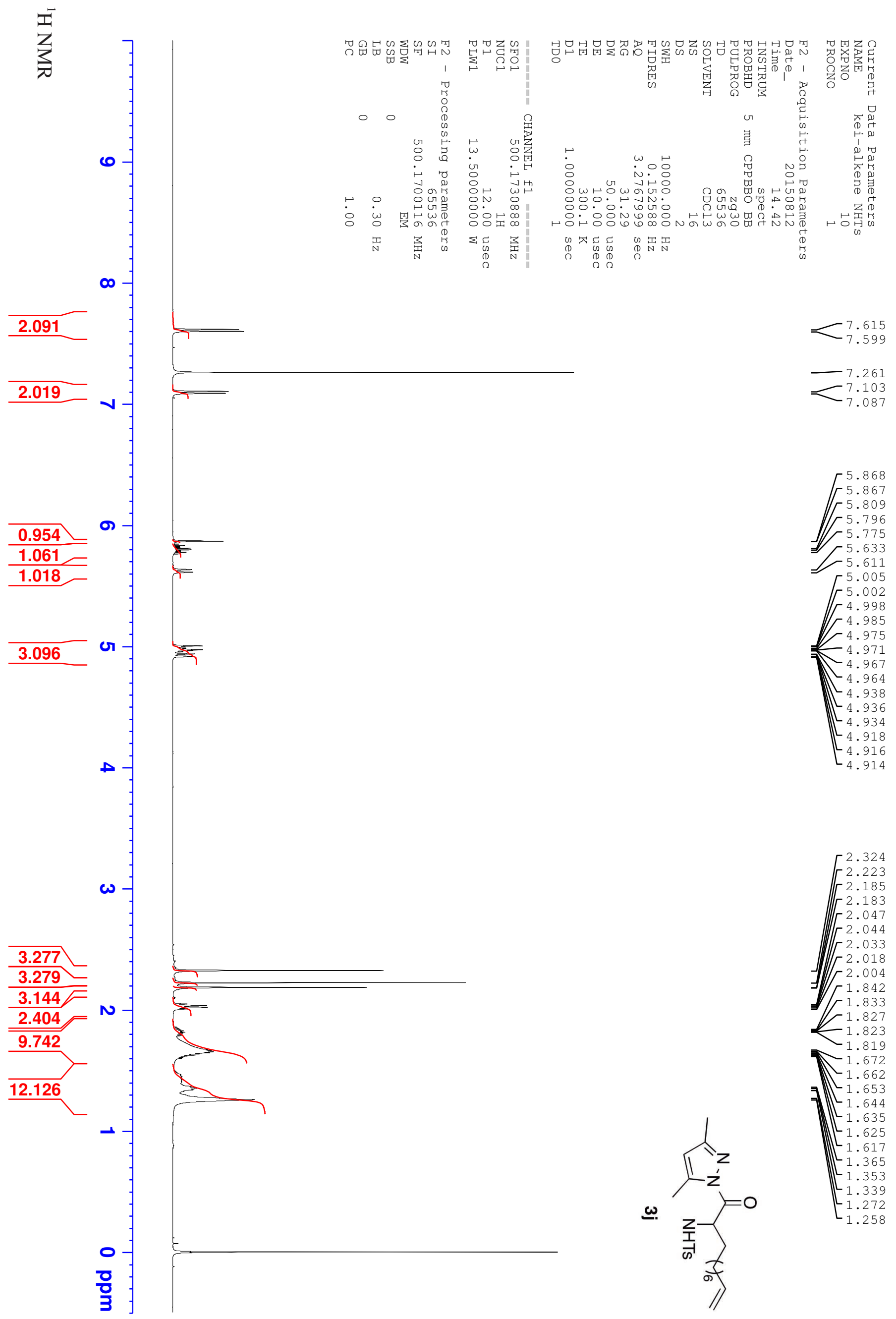


$\overbrace{}^{2}$

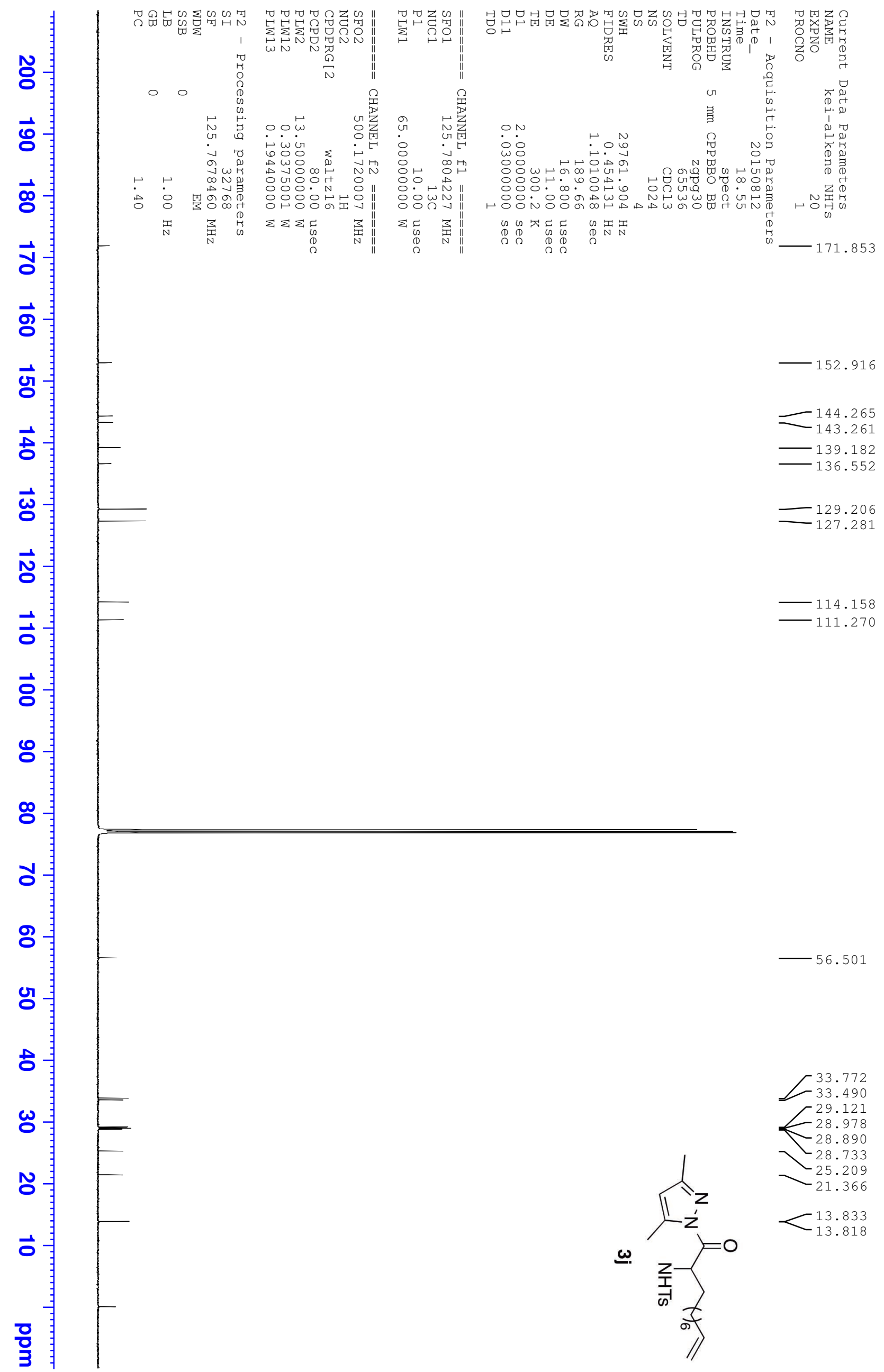




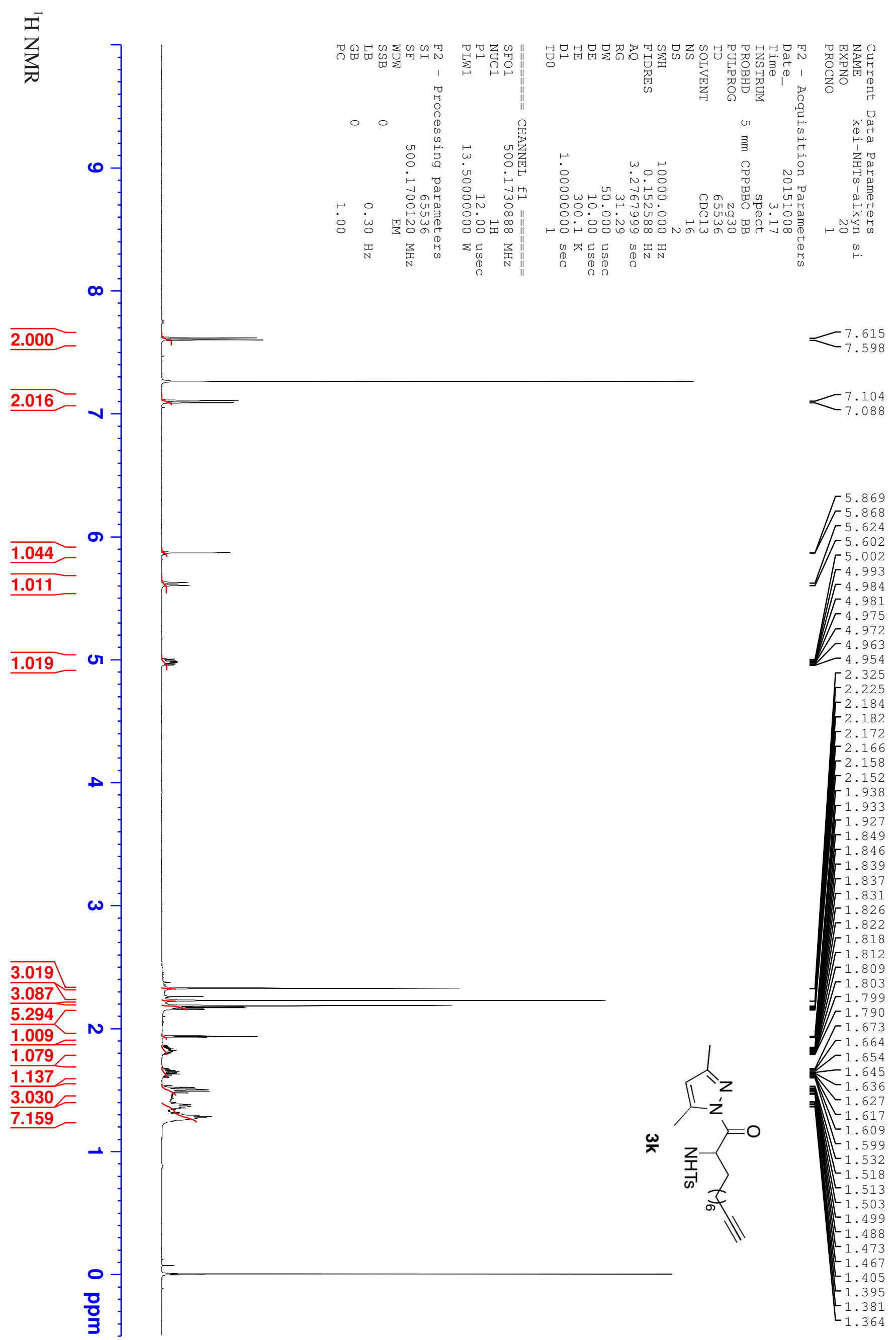


$\overbrace{}^{2}$

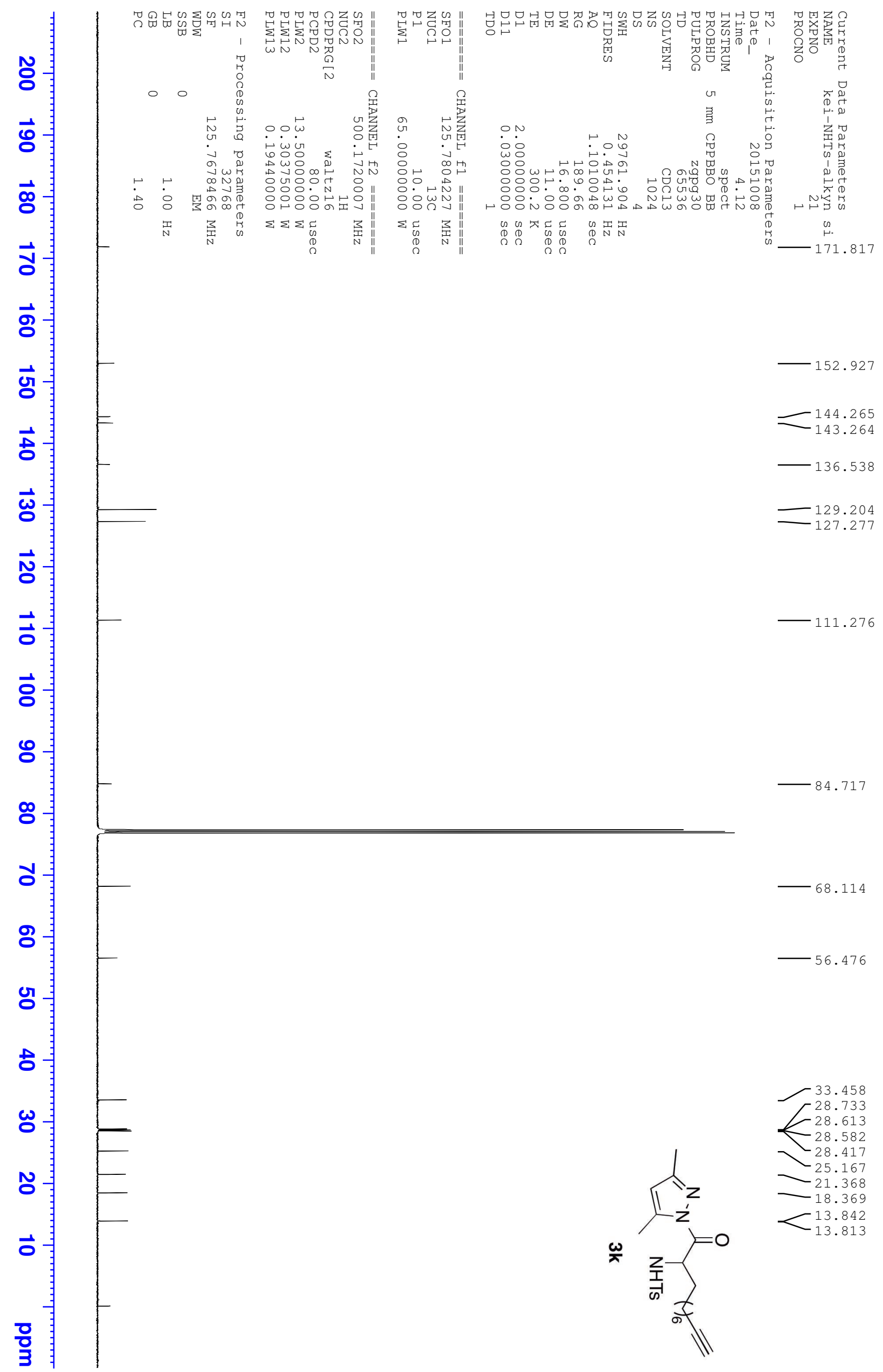



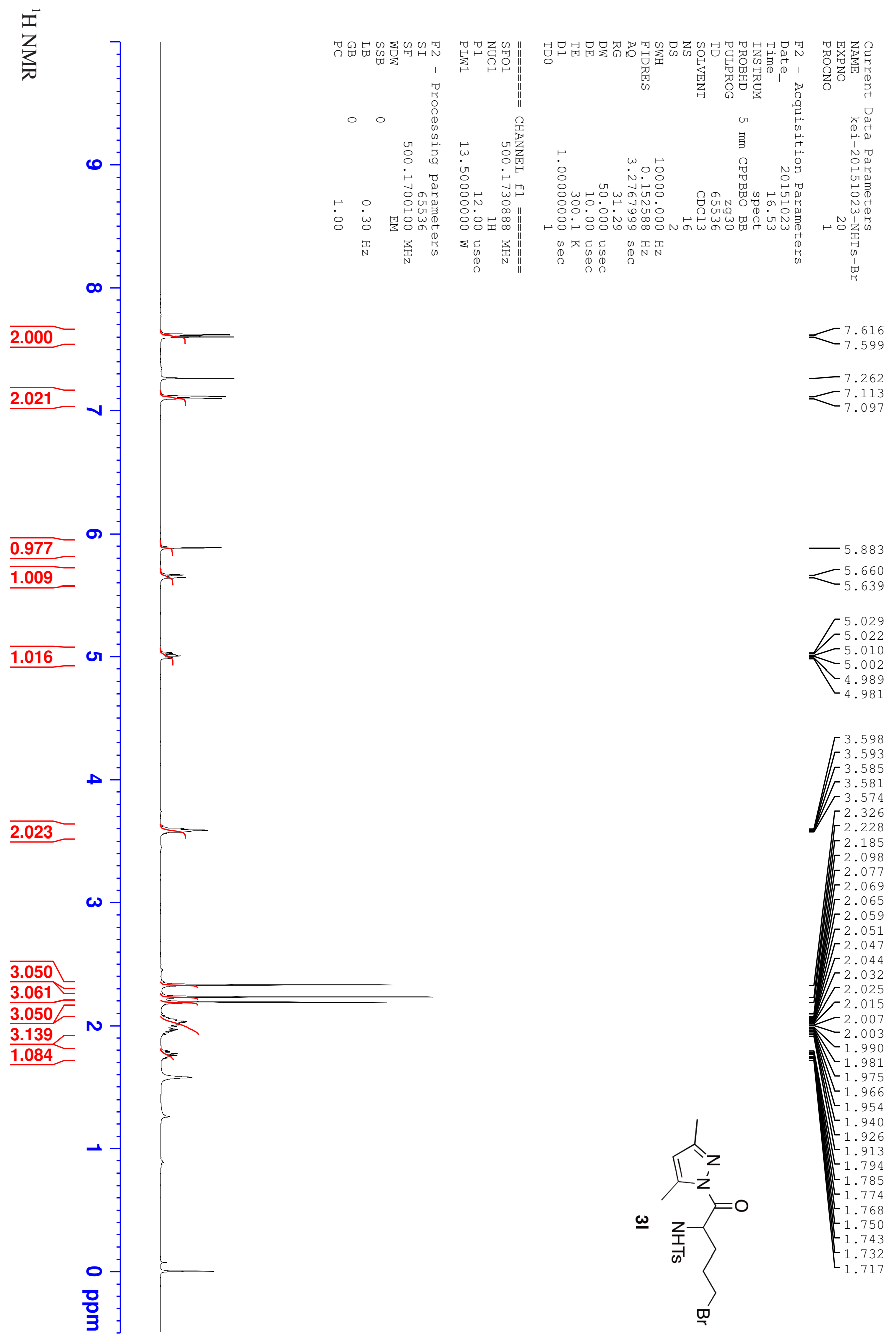
$\overbrace{}^{\bar{\omega}}$

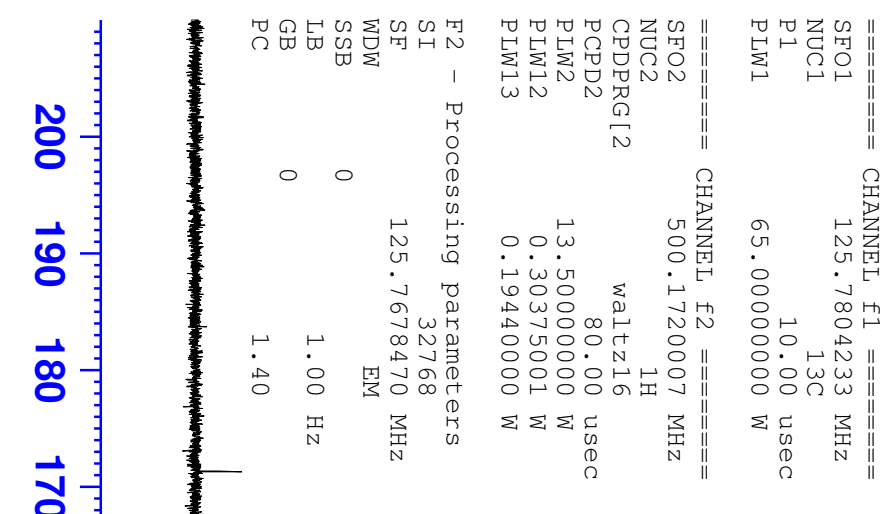

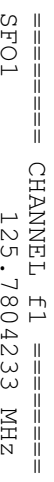

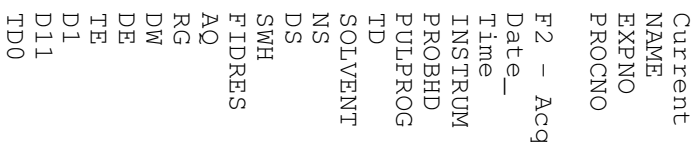

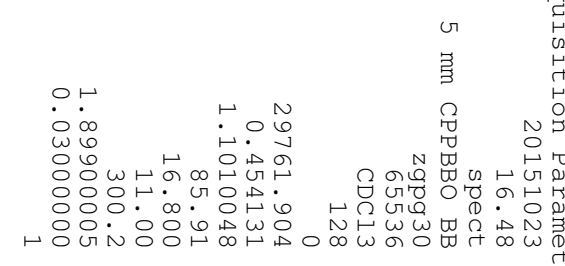

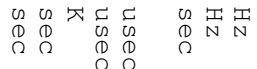

$\vec{\circ}$

ज्ञ

$\vec{f}$

$-144.318$

143.482

$-136.279$

$\vec{\omega}$

$\vec{N}$

$\vec{a}$

$\overrightarrow{\mathrm{s}}$

8

ฌ

$\infty$ 年

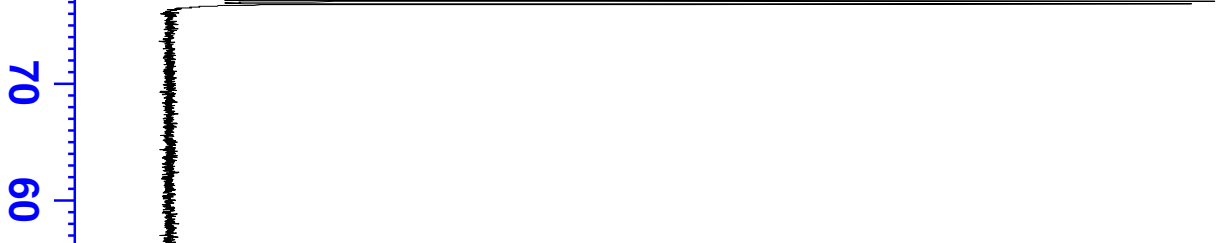

$-77.265$

77.011
76.758

8

gr

t

ผ

$\omega^{\prime} \quad$

30.813

28.502

ก

บ

$\overline{1}$

$\overrightarrow{0}$

응

苇 


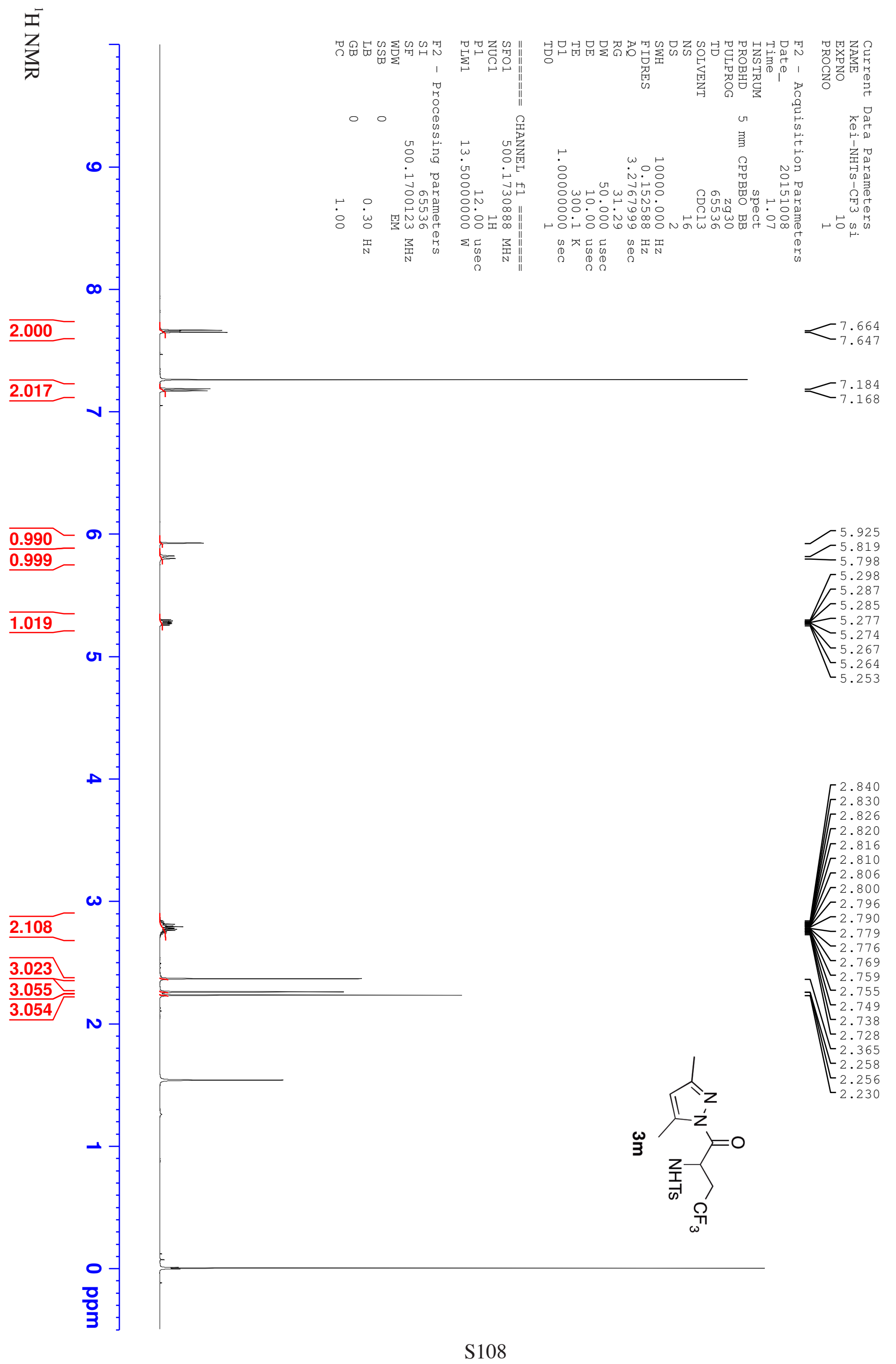


$\overbrace{0}^{\bar{\omega}}$

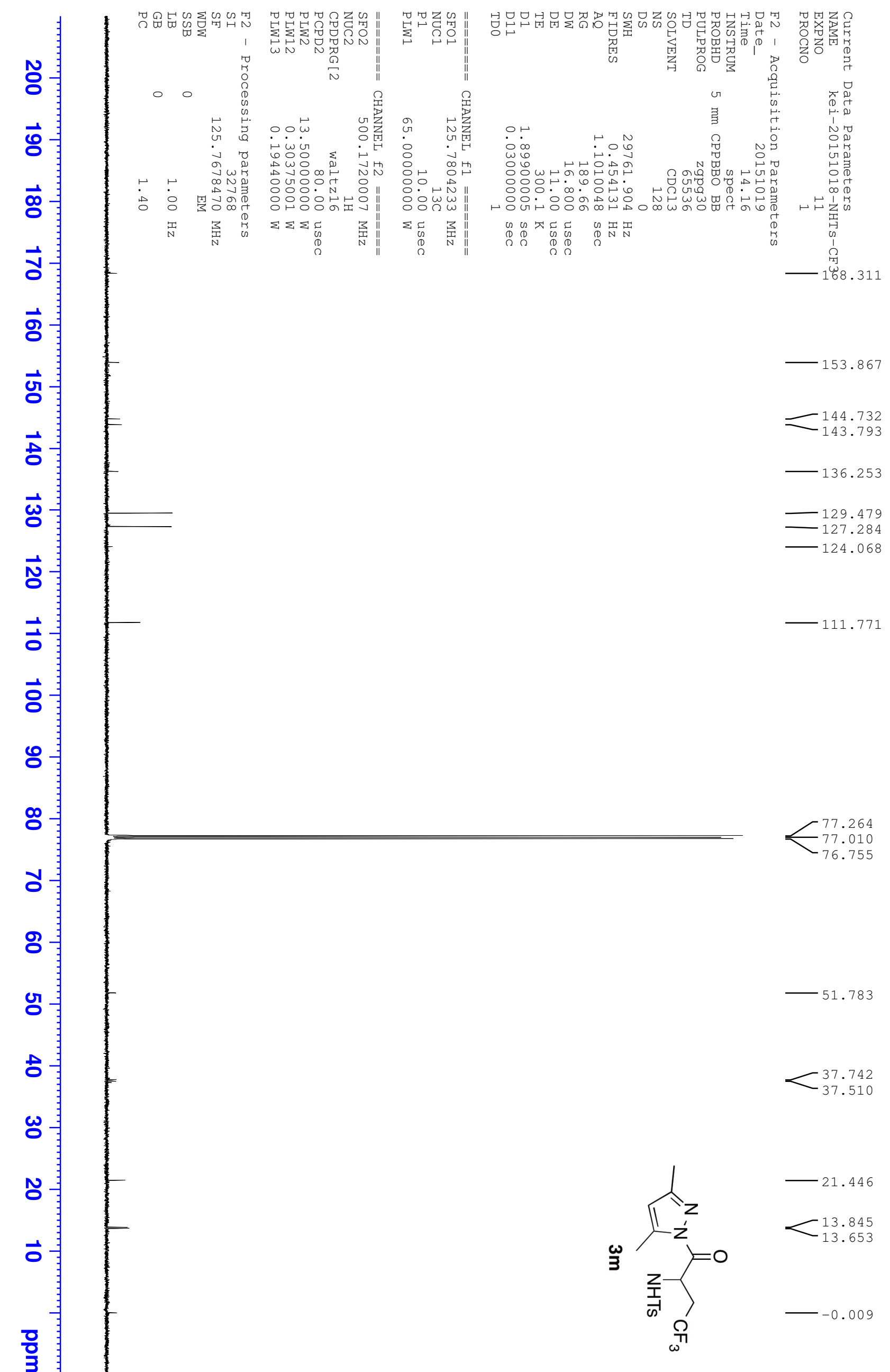




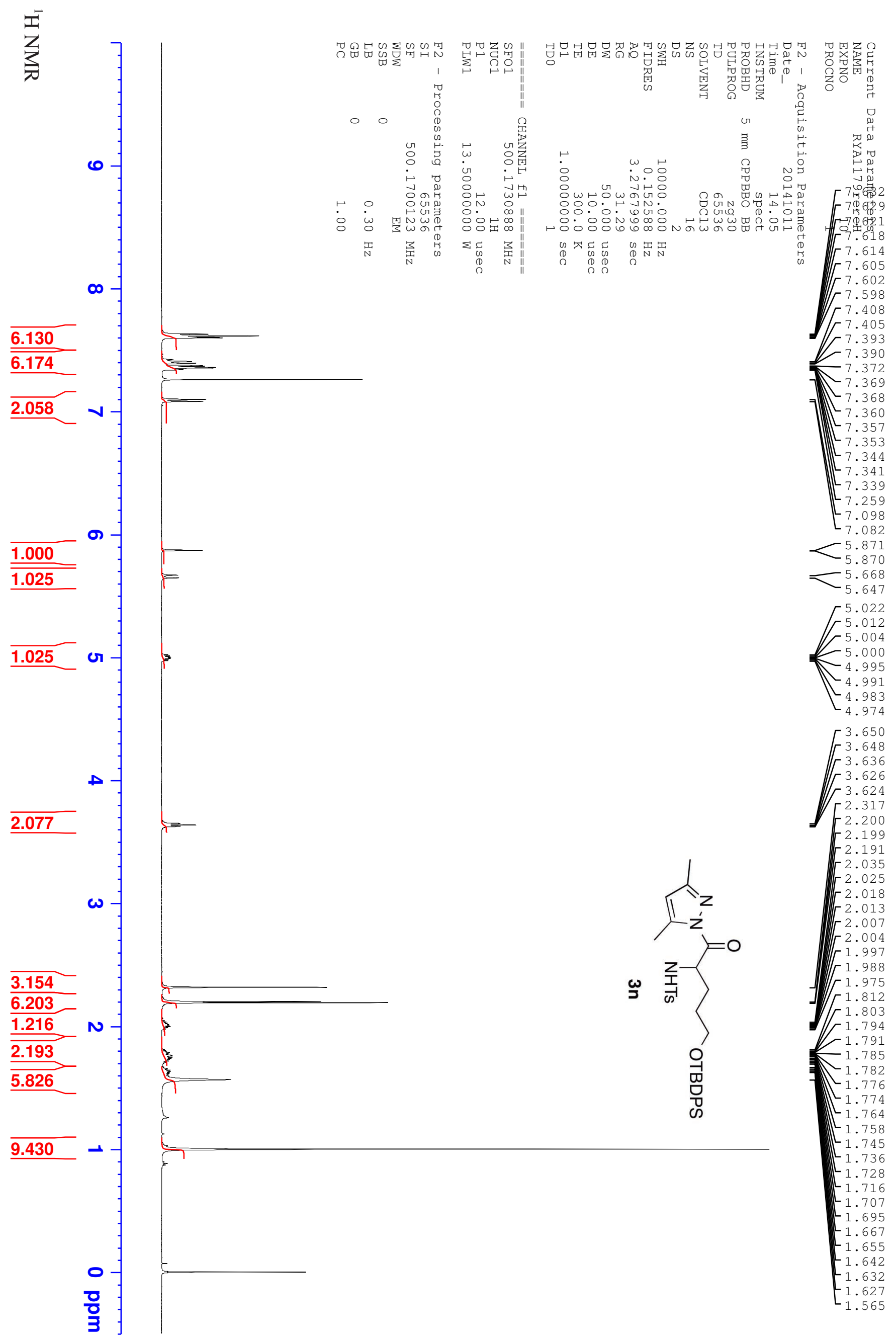


$\overbrace{}^{\bar{\omega}}$

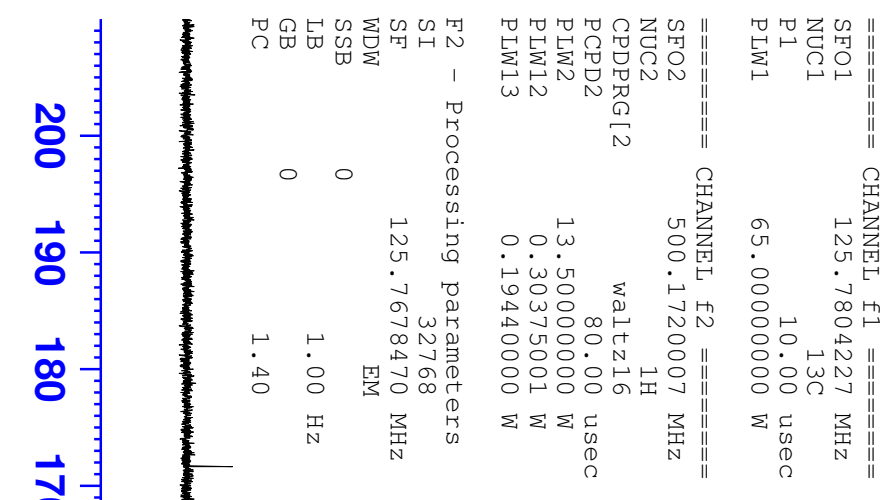

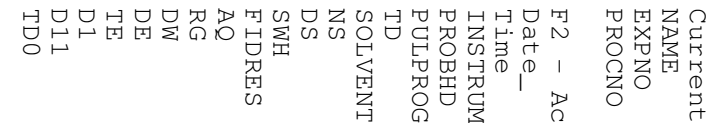

$\begin{array}{lll} & \\ & \end{array}$

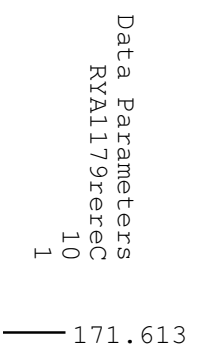

$\overrightarrow{8}$

ज़

$\overrightarrow{0}$

$\vec{\omega}$

E

ज

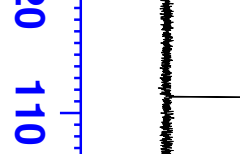

$\overrightarrow{8}$

๕

$\infty$

ชั

용

章

잉

s

w

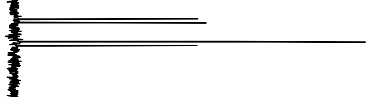

152.969

$-144.260$

143.281

$-136.530$

135.548

133.834

133.834
-129.229

129.229
-127.612

$\longleftarrow 127.612$

127.271

年

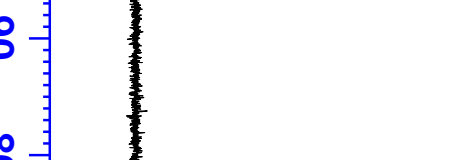

$-111.303$

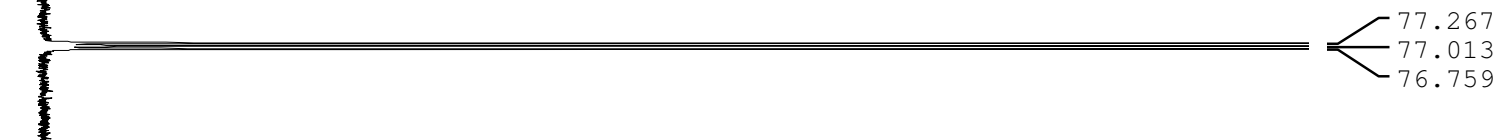

ก

ก

o
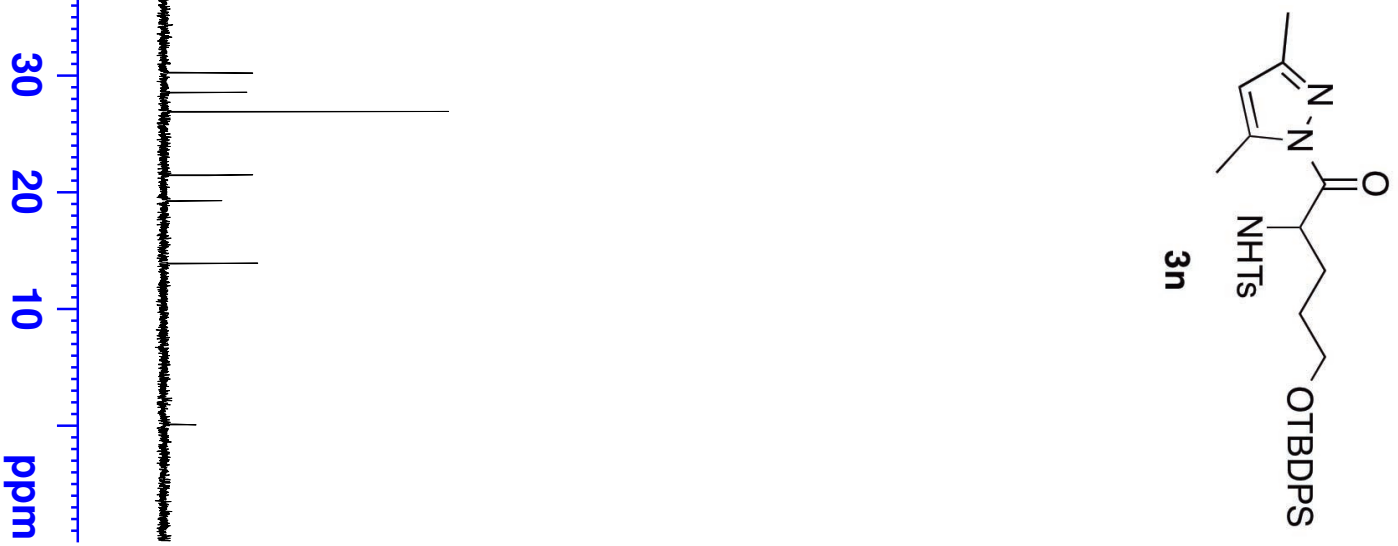

$-30.132$

$\begin{array}{r}-30.132 \\ -28.41 \\ \hline\end{array}$

21.372

$-19.166$

$+13.830$

13.805

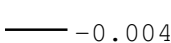



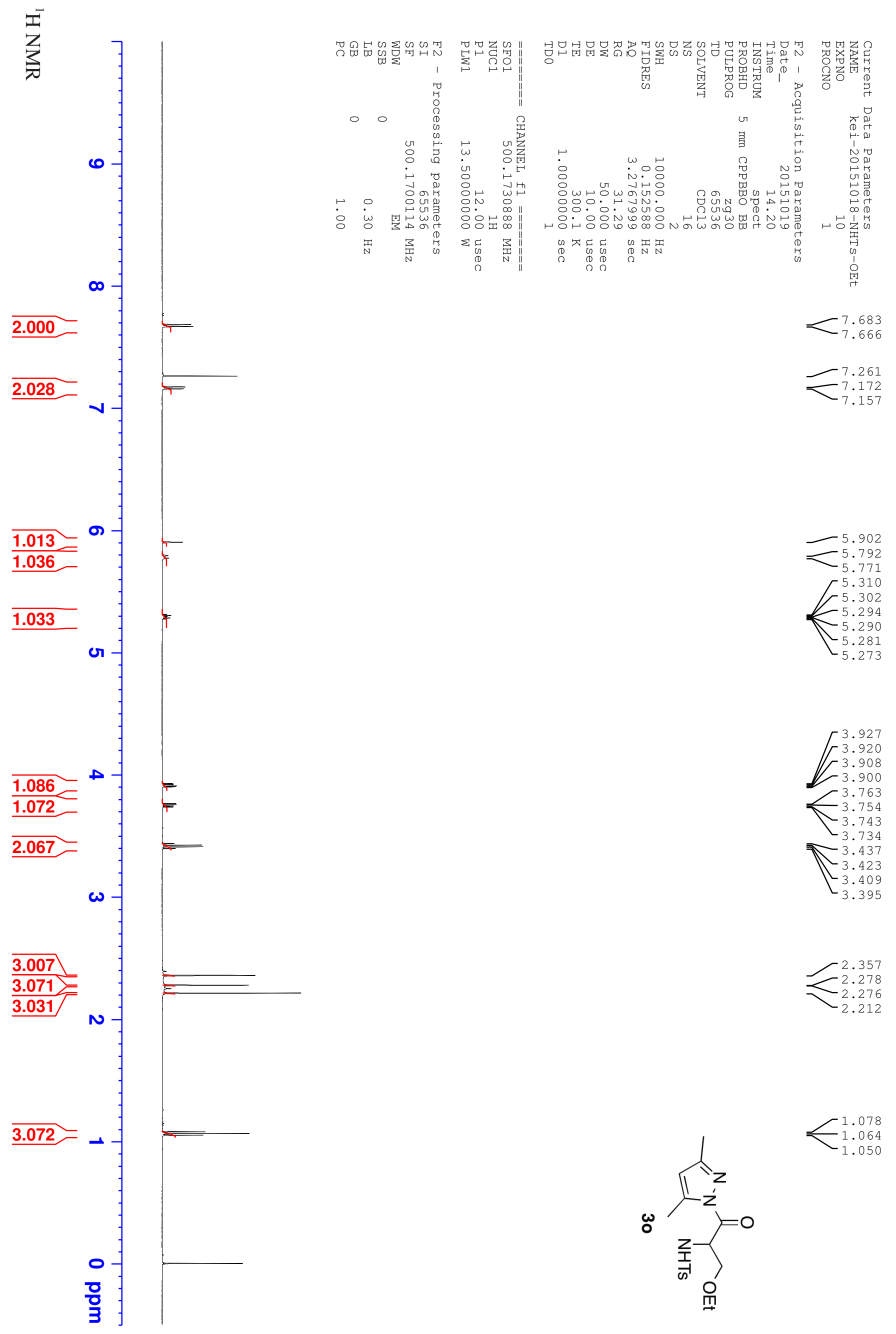

$-5.902$

$\widetilde{\gamma} .792$

5.310

5.302
-5.294

5.294
-5.290

5.290
-5.281

5.273

3.927

$F^{3.927} .920$

3.908
-3.900

3.900
-3.763

3.763
-3.754

$-3.743$

$-3$.

$-3.423$

3.409

3.395

$-2.357$

2.357
2.278

$-2.276$

2.212 
$\overbrace{}^{2}$

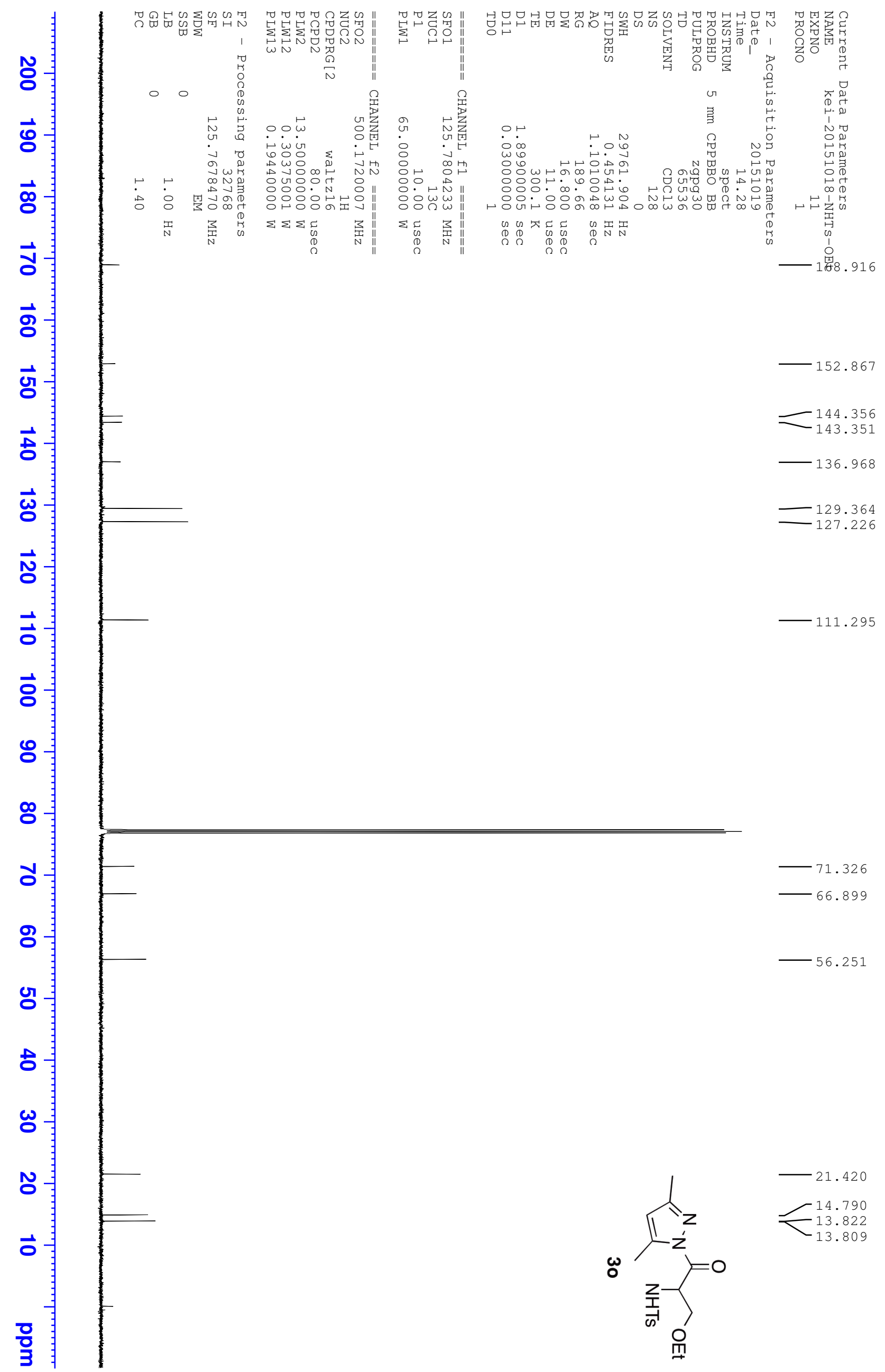



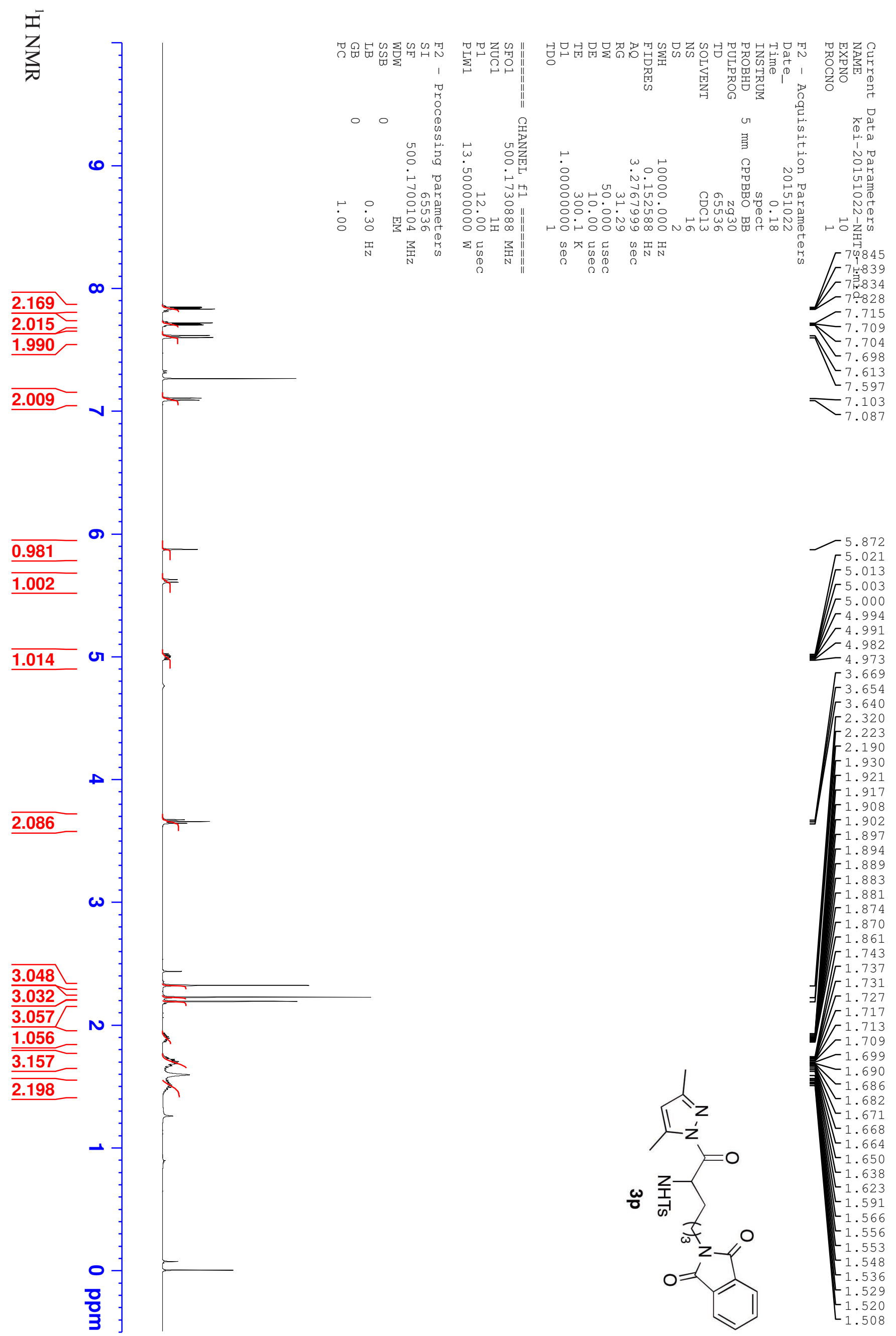
$\overbrace{0}^{\bar{\omega}}$
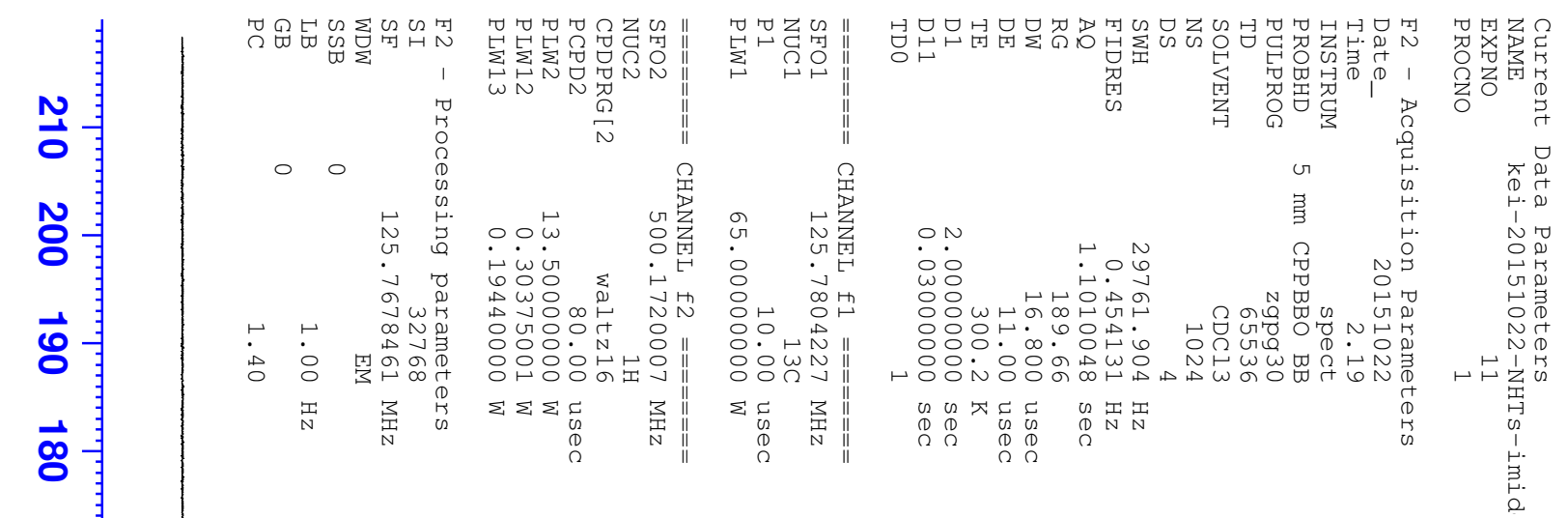

$\vec{\delta}$

$\vec{\circ}$

ज़

古

$\vec{\omega}$

$\vec{N}$

$\vec{\partial}$

$\overrightarrow{8}$

8

ø

ปั

8

잉

to

홍

$\omega$

ธ

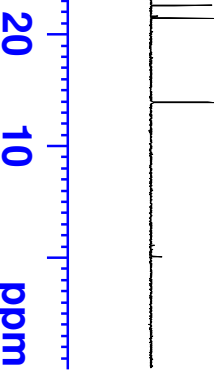

171.562
-168.371

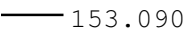

$-144.286$

$-143.321$

$-136.486$

$-133.879$

$-132.159$

$-127.289$

123.212

青

|

$-111.392$

$-56.168$

용

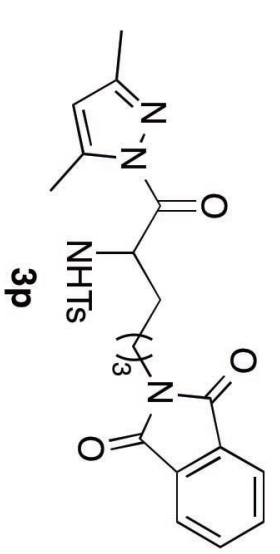

$-37.441$

$-32.798$

27.686

-22.499
-21.372

$<13.841$

$-13.802$ 


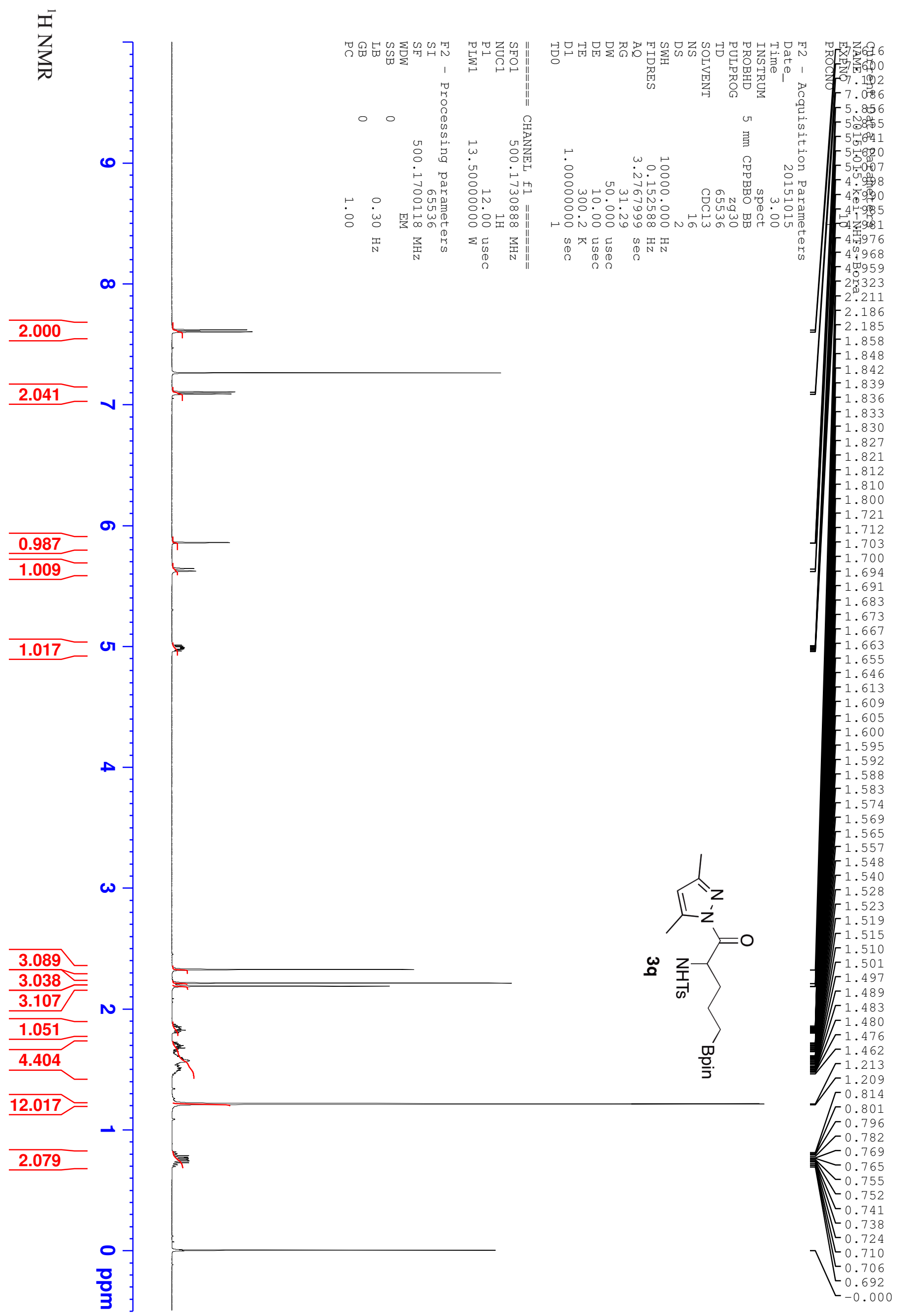


$\overbrace{}^{\bar{\omega}}$

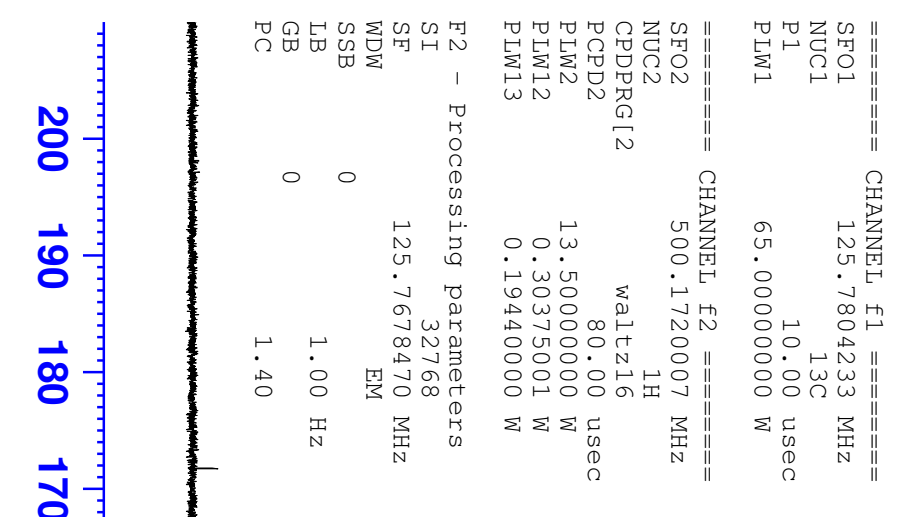

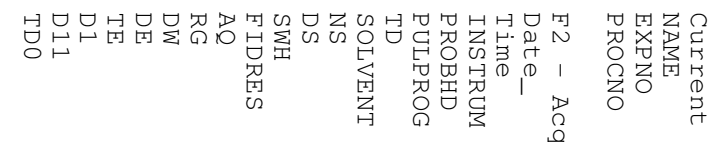

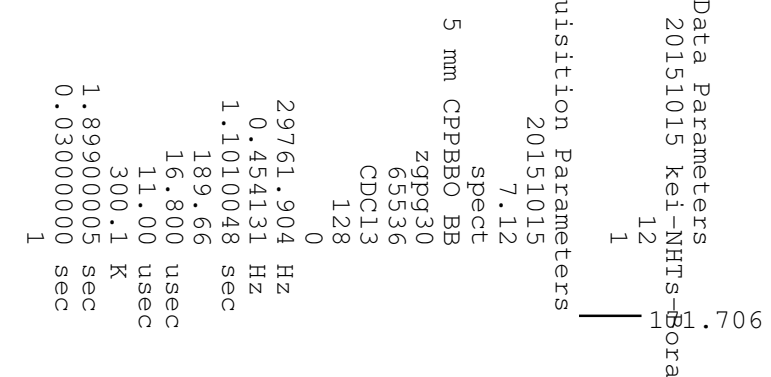

$\overrightarrow{8}$

방

$\overrightarrow{\mathrm{t}}$

144.233
-143.177

$-136.608$

$-129.188$

127.290

$\vec{\circlearrowleft}$

孛青

$-111.177$

$\overrightarrow{\mathrm{s}}$

8

ஃ

ชั

8

잉

잉

t

ผ

กั

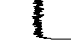

$-82.987$

$-56.584$

$-35.932$
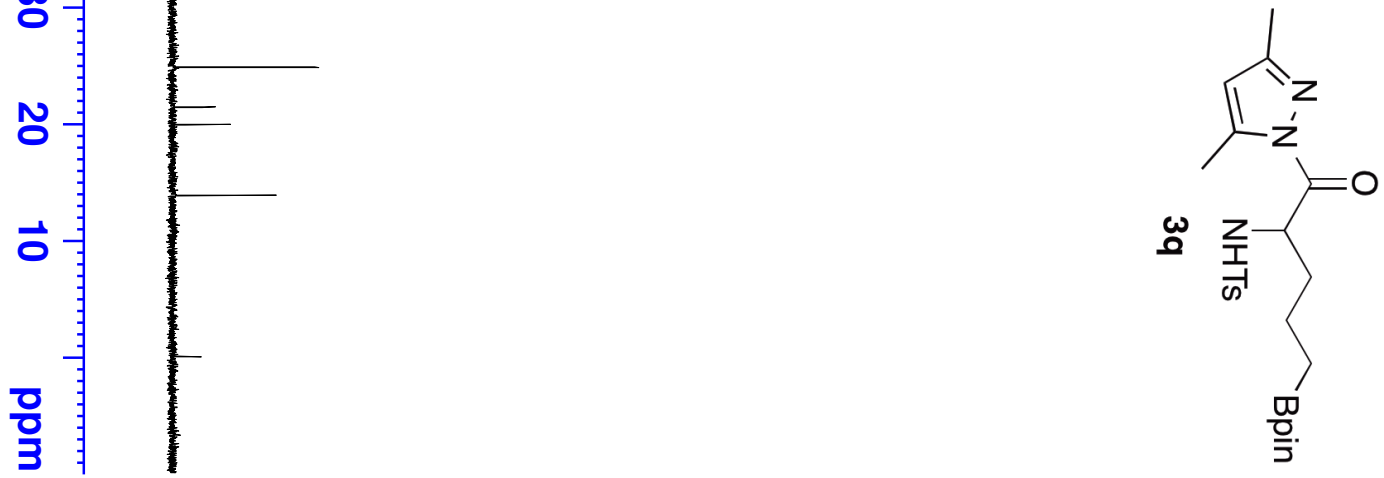

$-24.799$

$<24.786$

-21.368
-19.874

$-13.816$ 


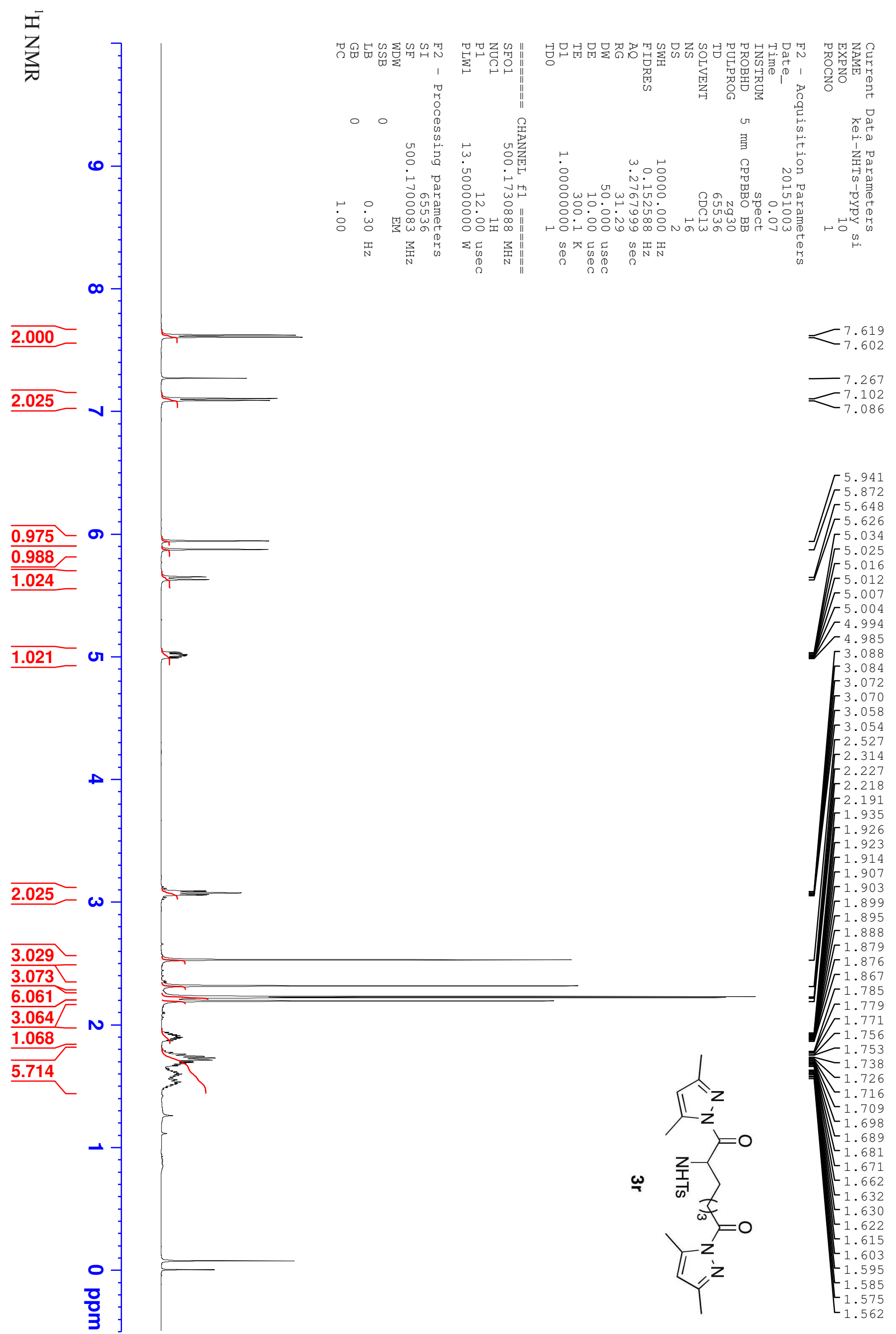


$\overbrace{}^{2}$

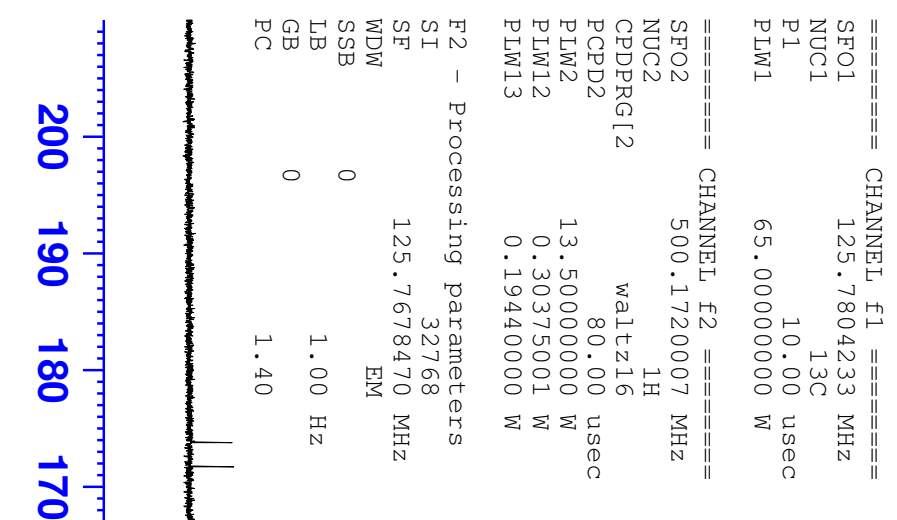

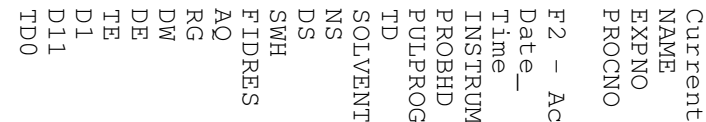

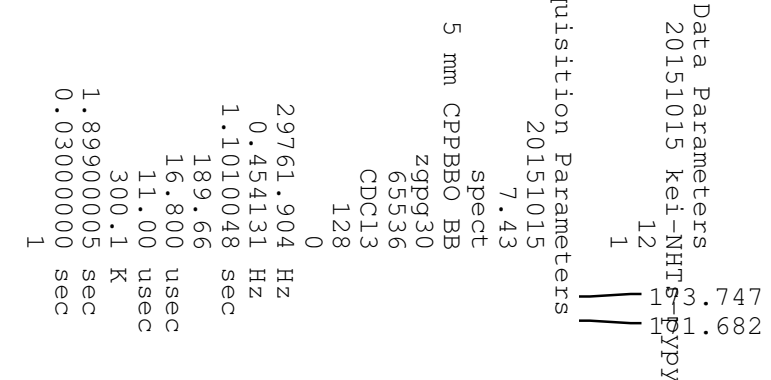

$\vec{\circ}$

ज़

$\overrightarrow{0}$

$\overrightarrow{\mathrm{O}}$

$\overrightarrow{0}$

$\overrightarrow{8}$

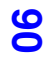

ஜ

ชั

ᄋ

당

b

ம

ง

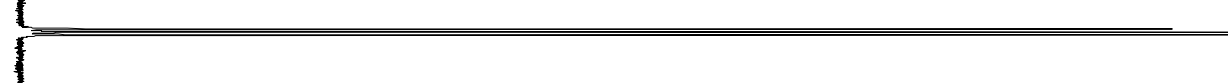




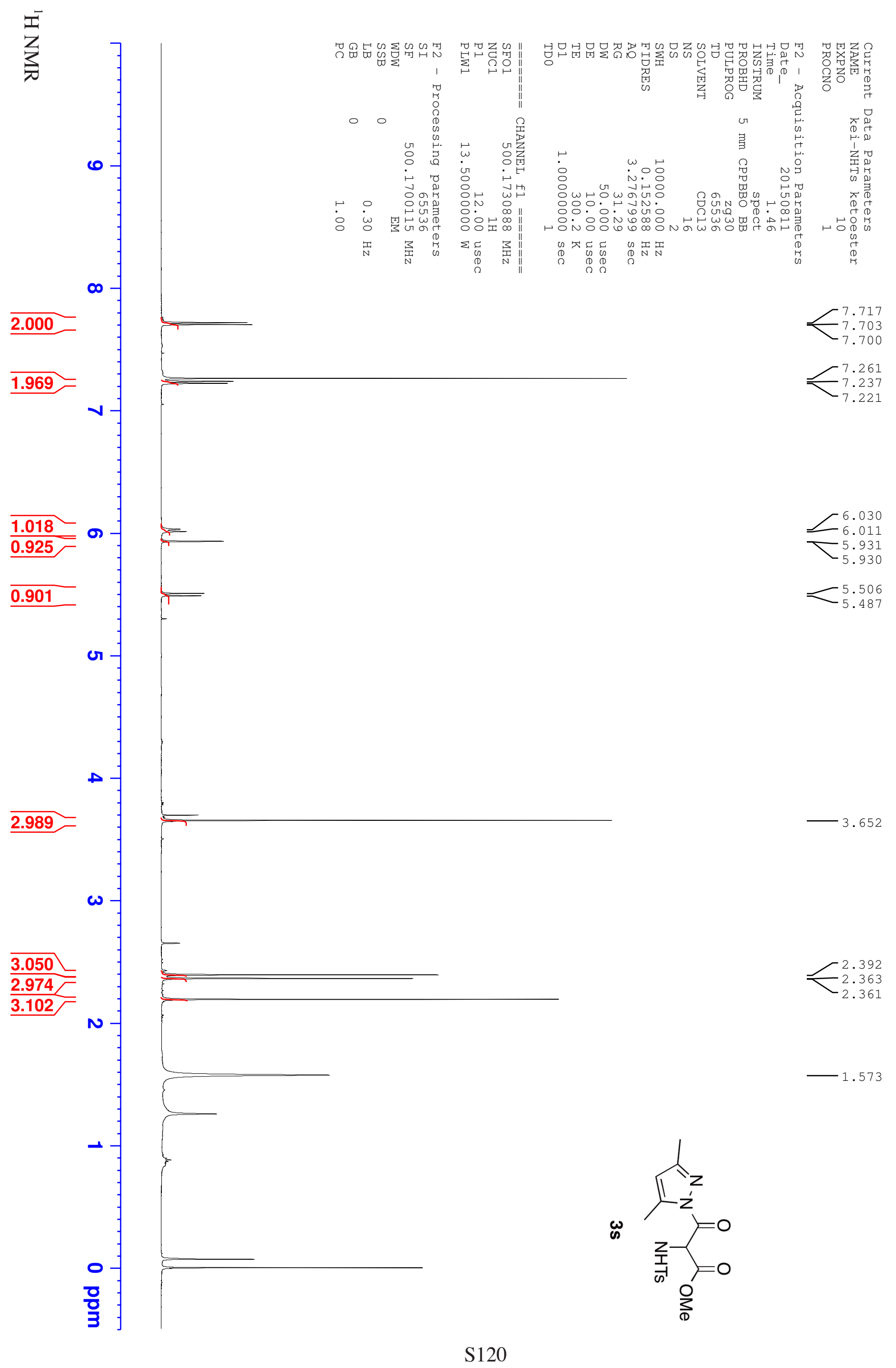


$\overbrace{}^{2}$

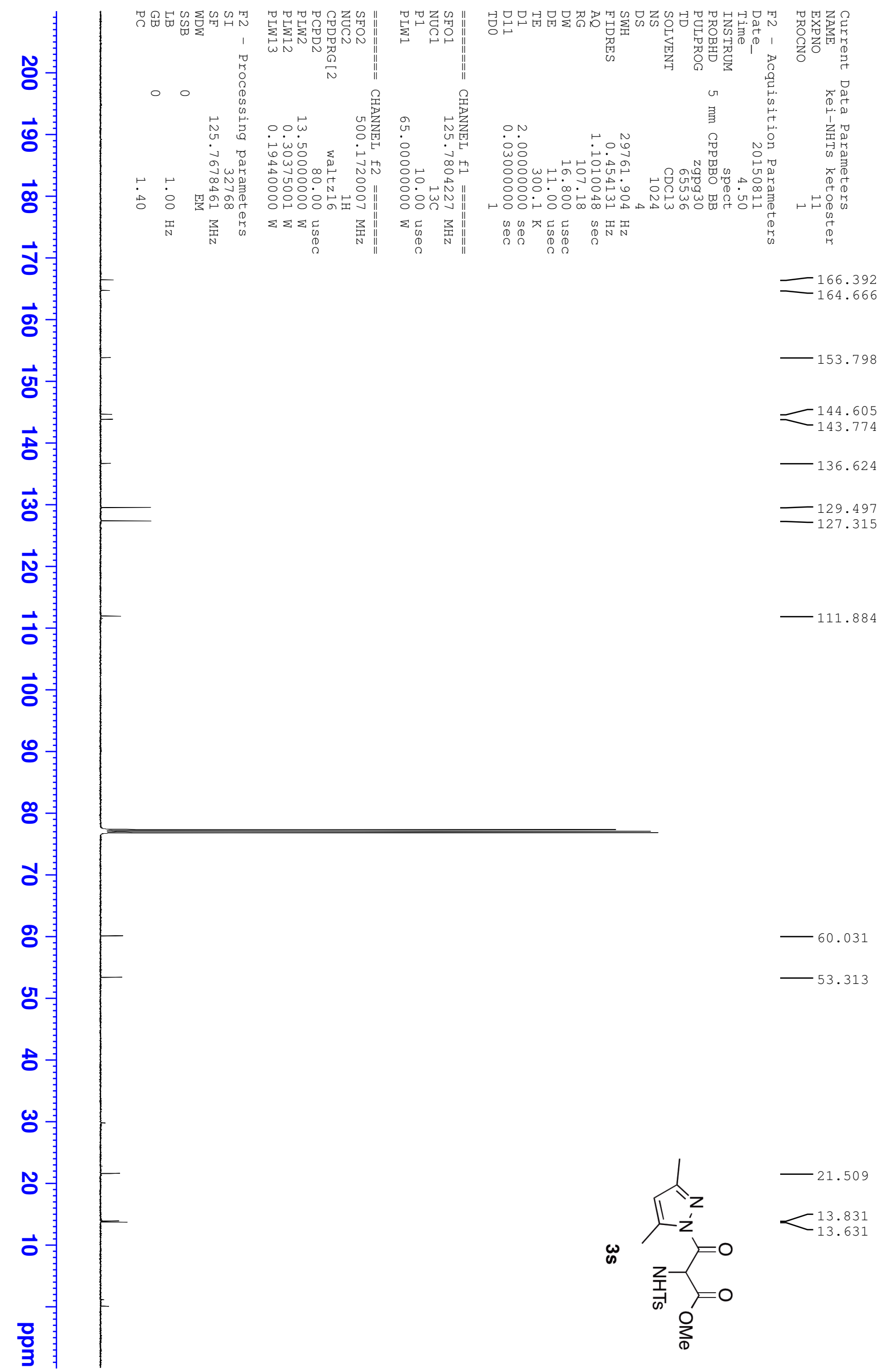




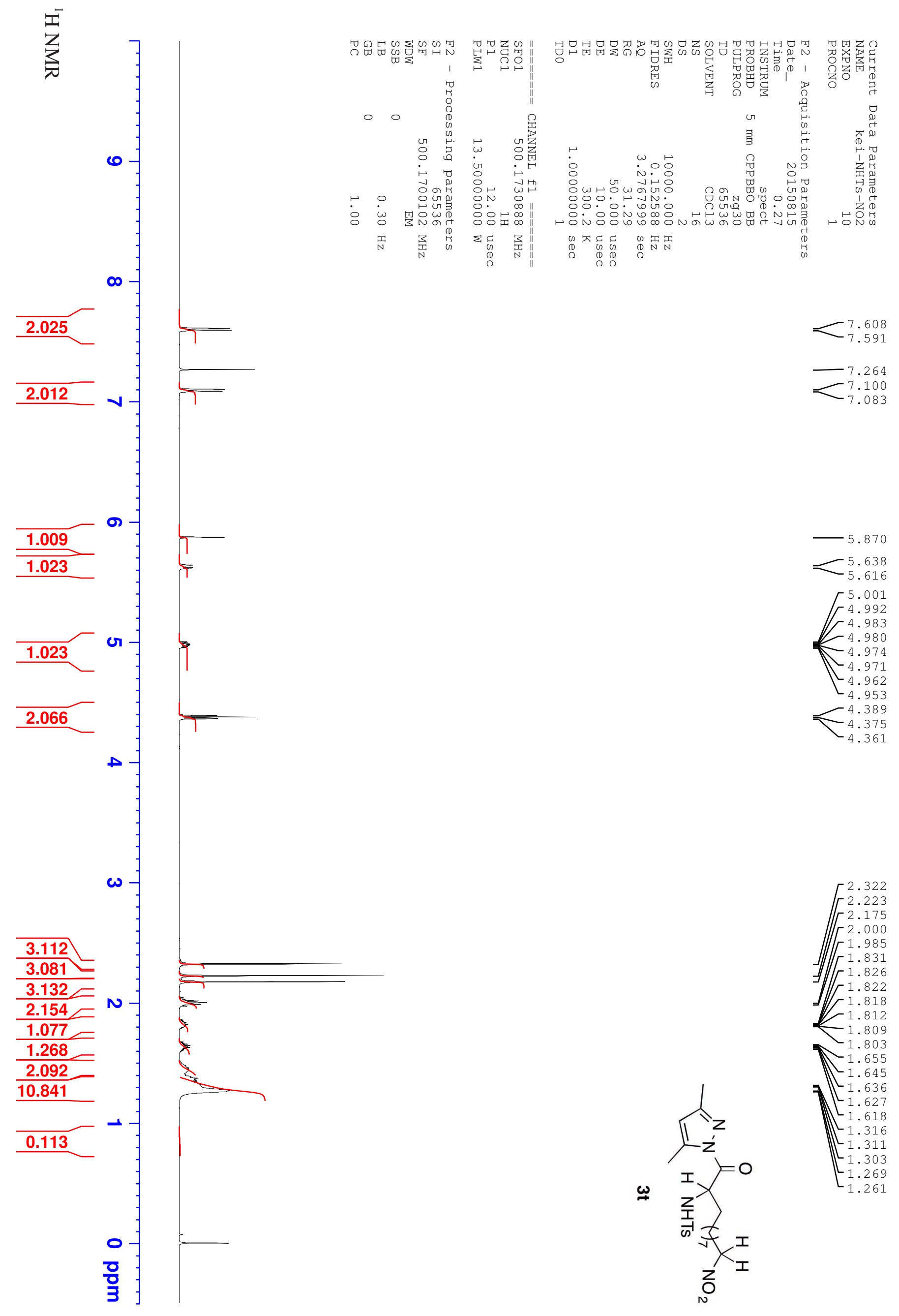


$\overbrace{}^{2}$

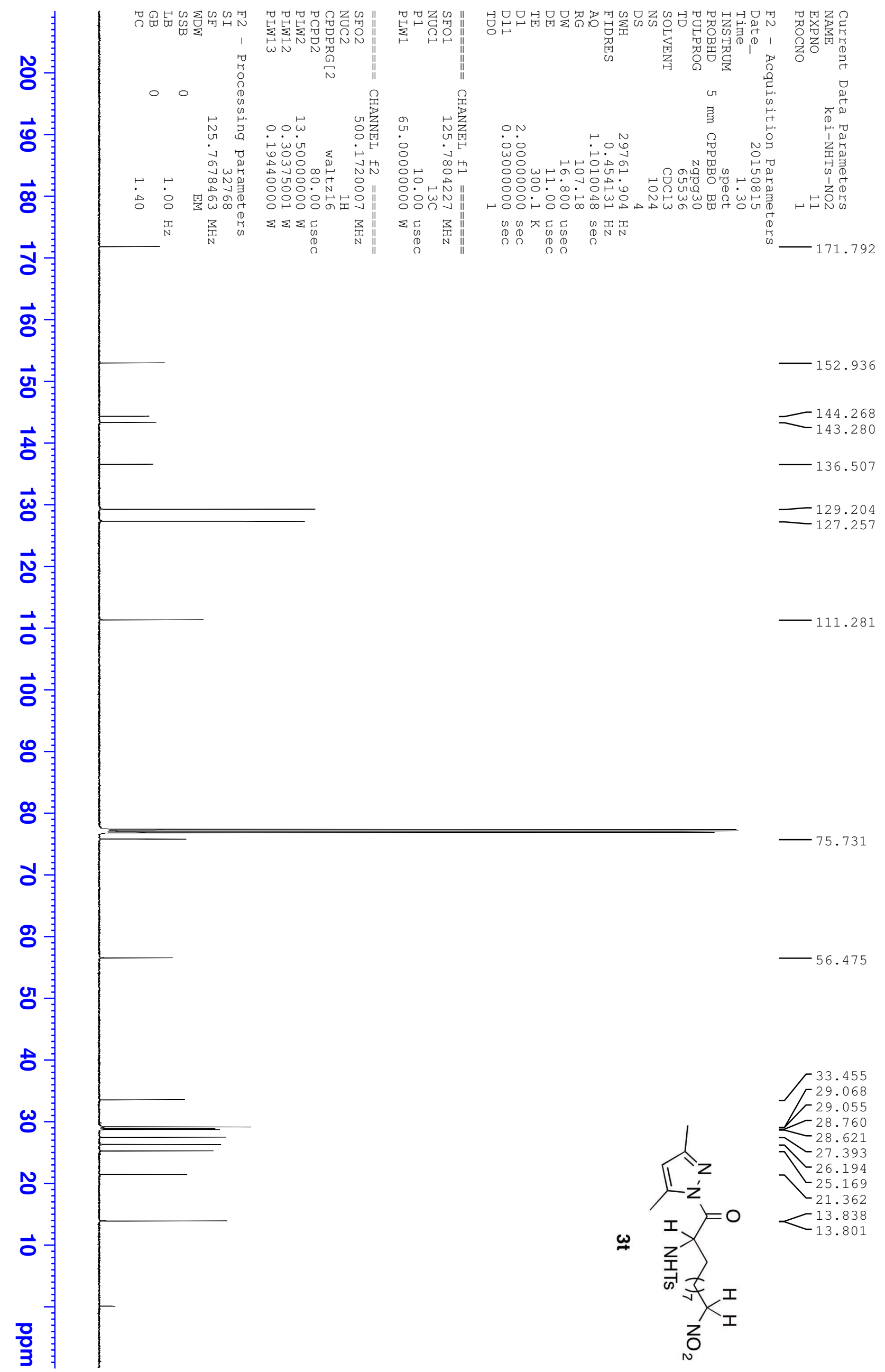




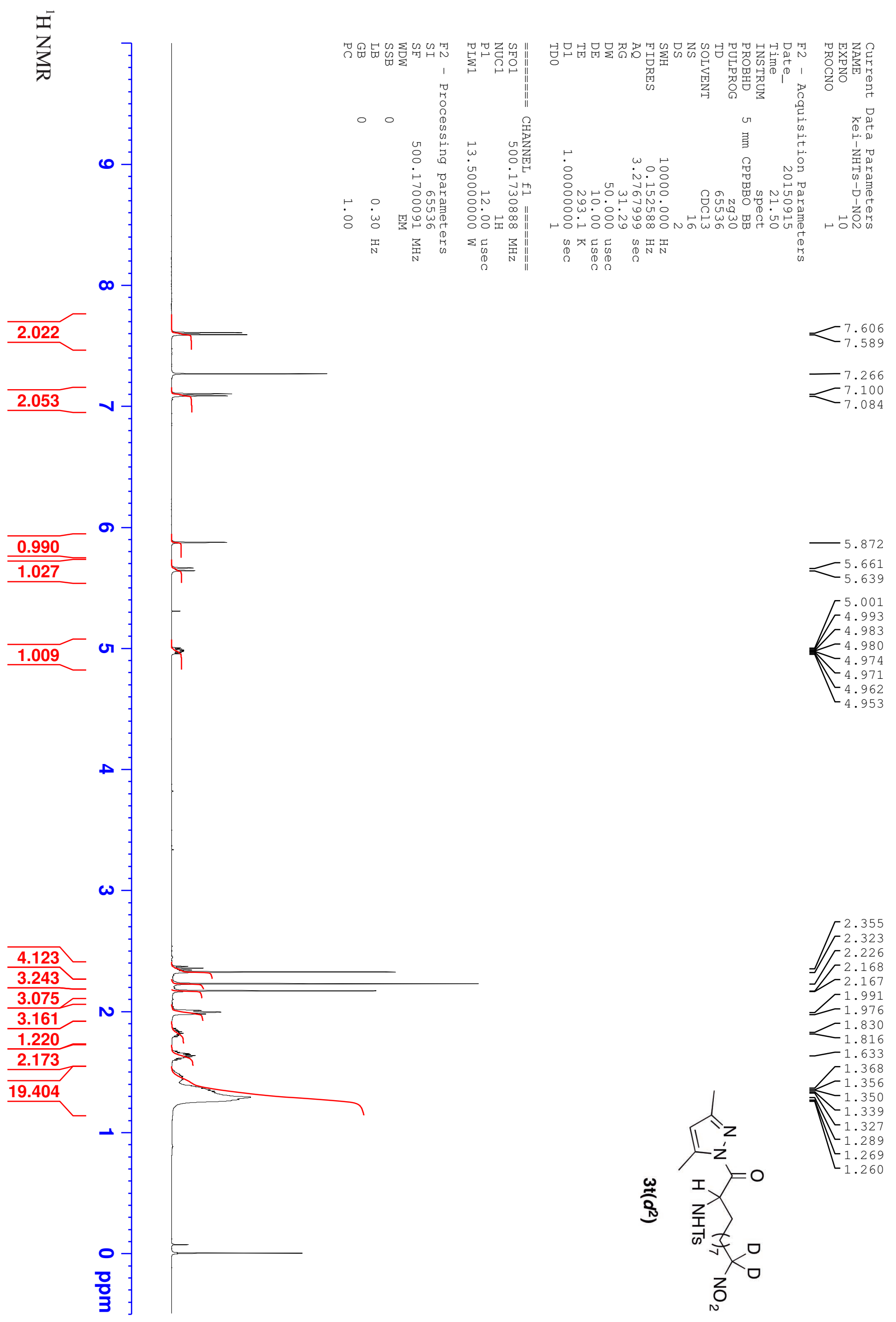


$\overbrace{}^{\bar{\omega}}$

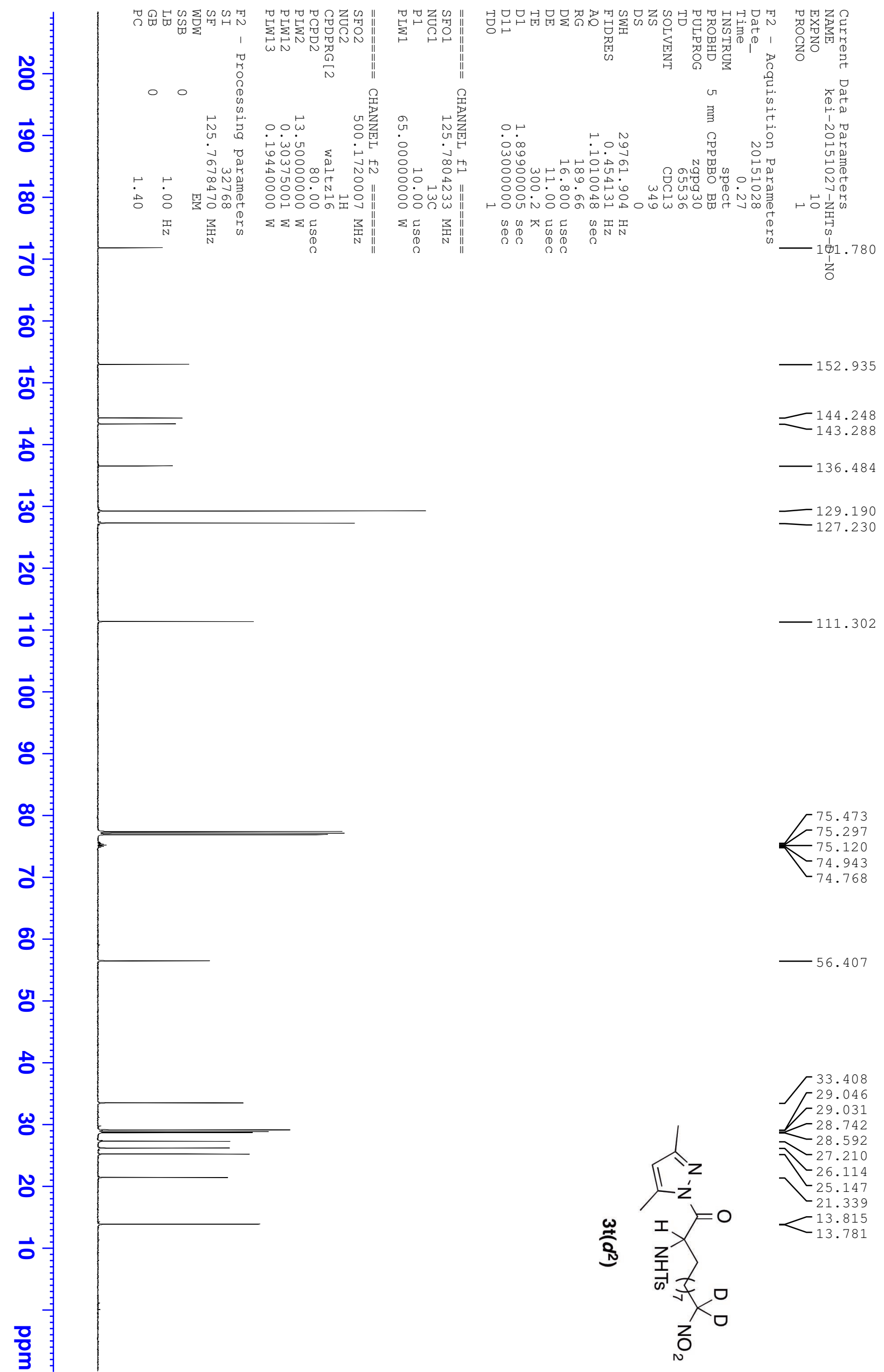



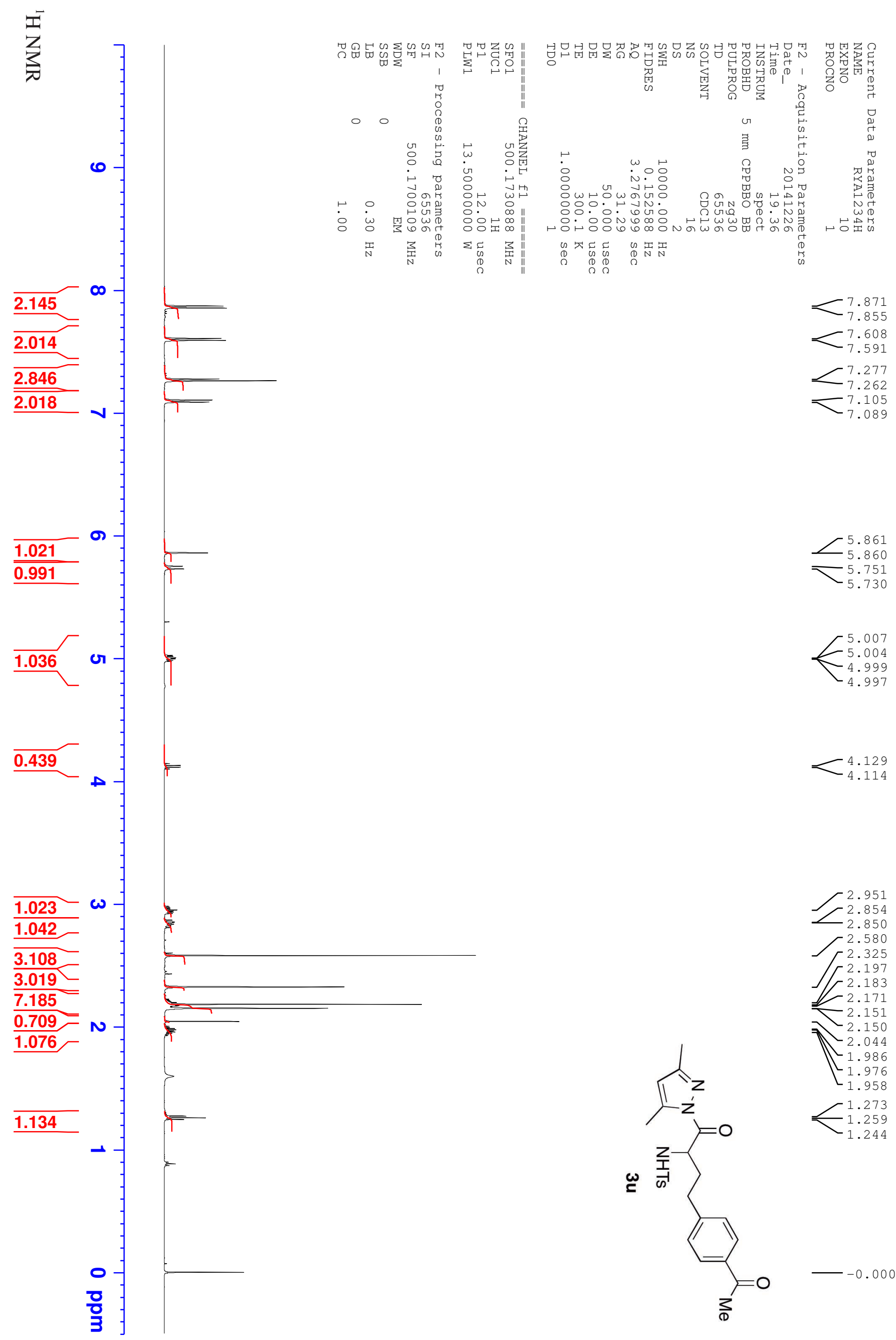

5.007

5.004

$-4.999$

4.997

$\leftarrow 4.129$

4.114

2.951

2.854

2.850

2.580

2.325

$r^{2.197}$

$r_{2.171}^{2.183}$

$-2.151$

$-2.150$

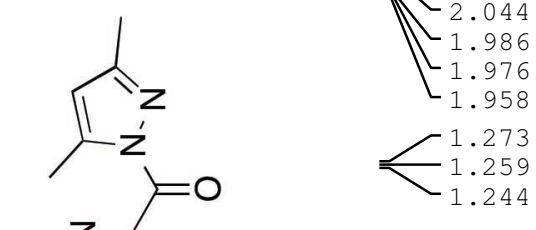


$\overbrace{}^{\bar{\omega}}$

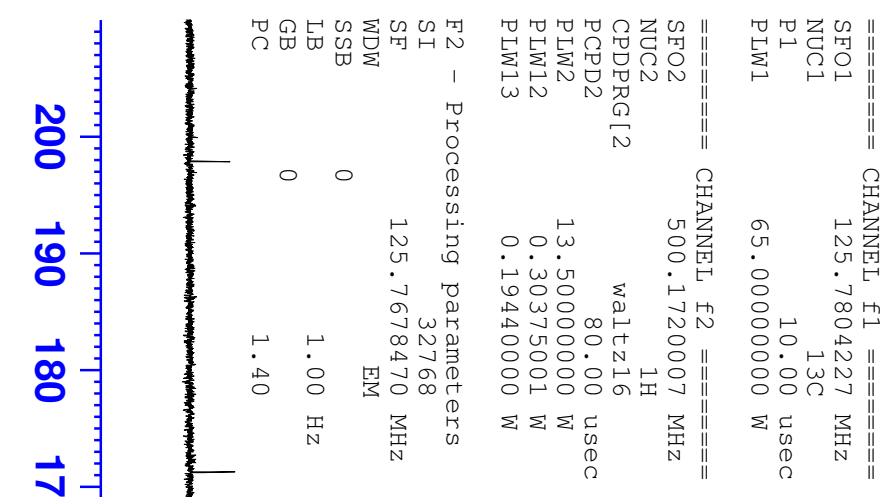

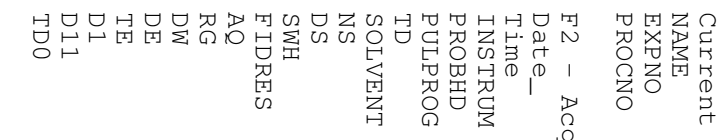

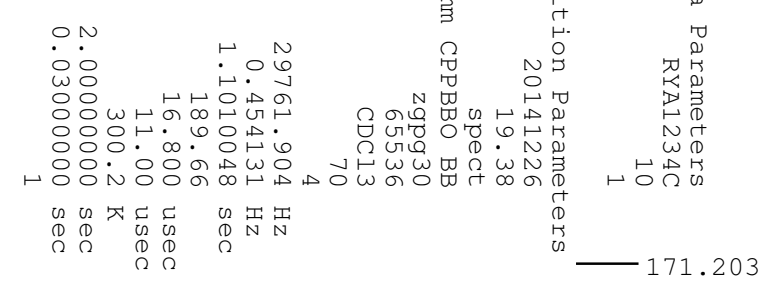

- 153.202

जू

흥

के

ట్

$\overrightarrow{0}$

$\overrightarrow{\text { N }}$

$\overrightarrow{0}$

$\overrightarrow{8}$

8

ஜ

ป

용

잉

8

๘

$\mathbb{N}_{z}$

N
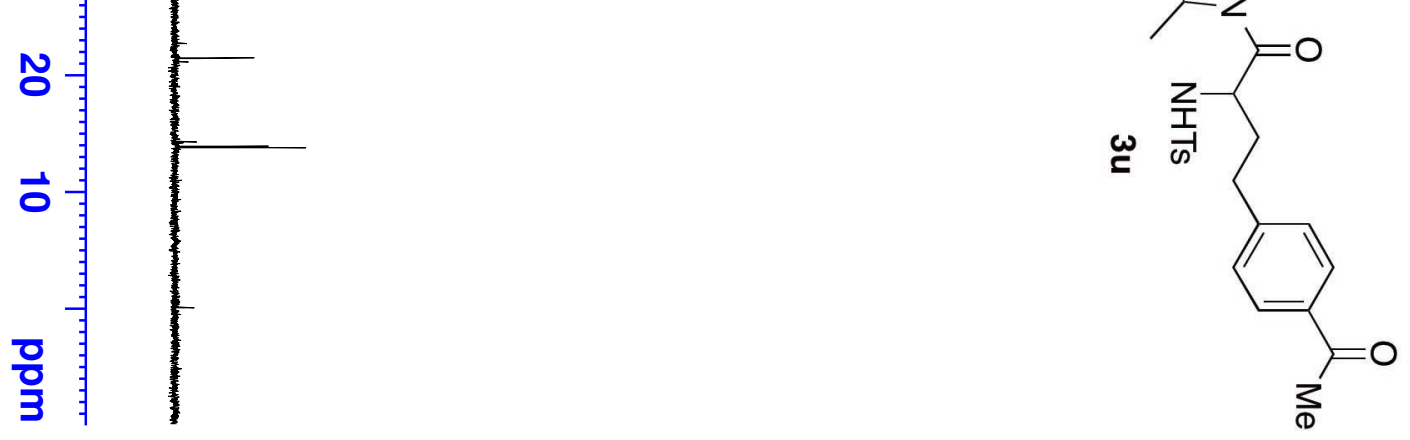

$-34.617$

$-31.627$

$-26.572$

$-21.377$

$+13.809$

$-13.717$ 

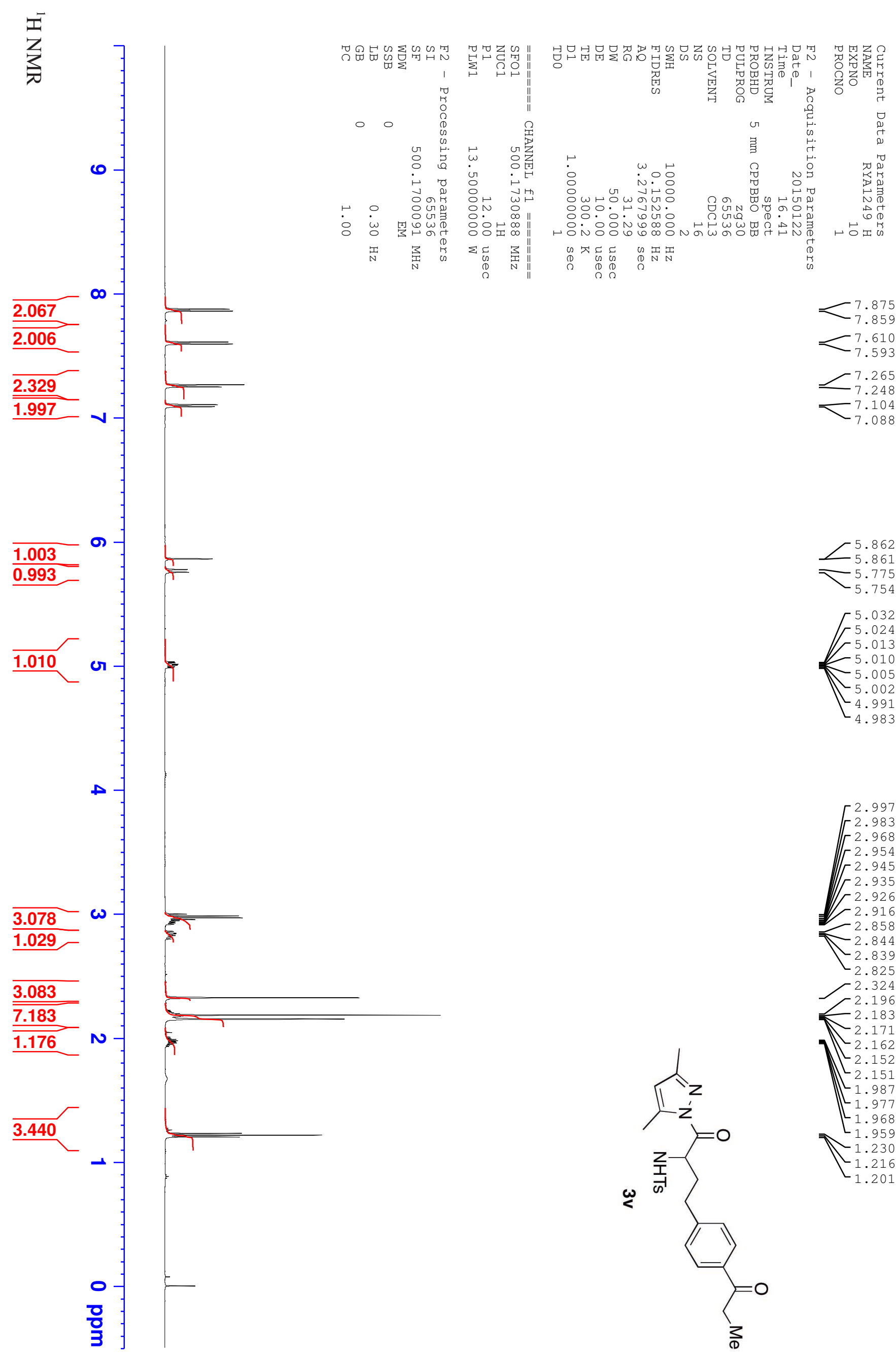

5.862 5.861

$-5.775$

5.754

$\Gamma .032$

5.024

5.013

5.010
-5.005

$-5.002$

$-4.991$

4.983

2.997

2.968

2.954

I 2.945

2.935

$F^{2.935}$

2.916

2.858
-2.844
-2.839

$-2.839$

2.825

$-2.324$

2.196

2.183

$\mathbb{L}-2.171$

$-2.152$

2.151

L 1.987

- 1.977

1.968

1.959

$-1.216$

1.201

๗

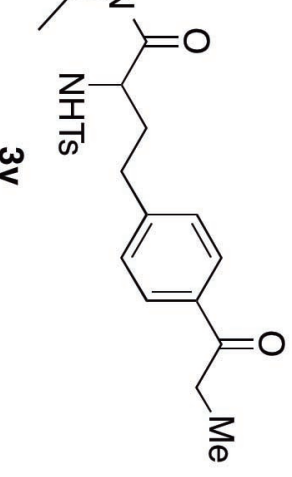


$\overbrace{}^{2}$
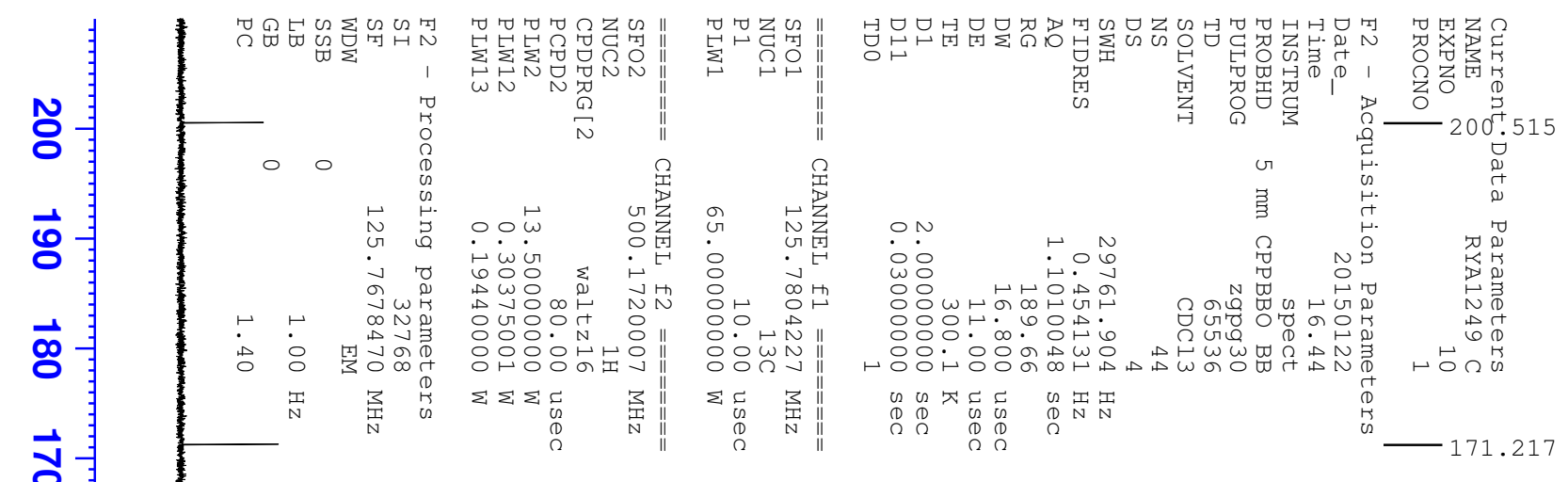

홍

ज्ञ

룽

$\overrightarrow{\mathrm{O}}$

$\vec{a}$

$\overrightarrow{8}$

$\mathscr{8}$

$\infty$

$\checkmark$

용

잉

뭉

ผ

ง

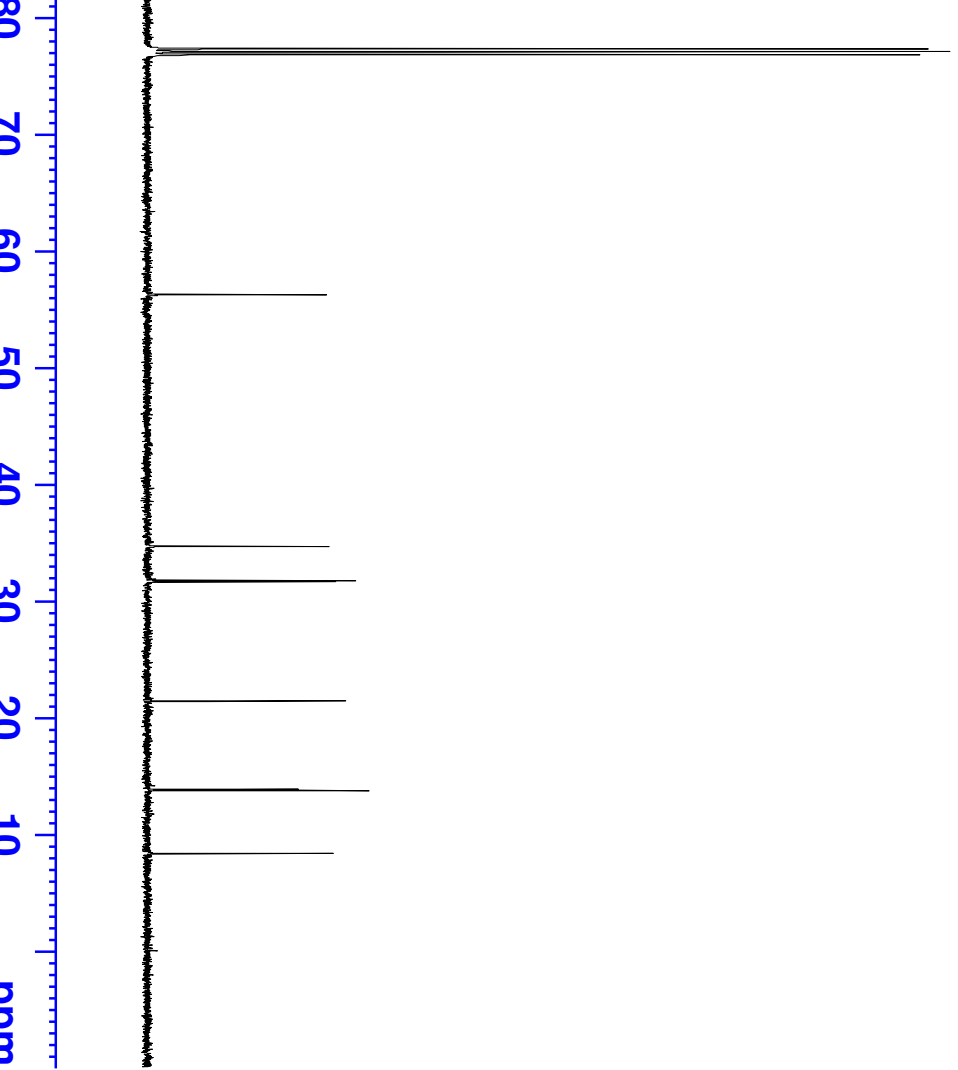

$-153.191$

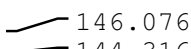

144.316

143.471

$-136.322$

$-135.043$

$-129.287$

$-128.787$

$\checkmark 127.272$

- 111.420

年

8

ㄱ․ㄱ

$-56.210$

o

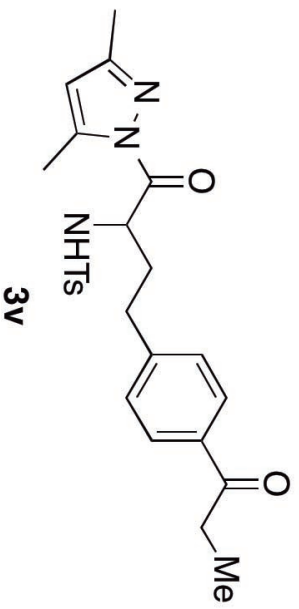

$-34.625$

$\leftarrow 31.706$

77.037
76.783 


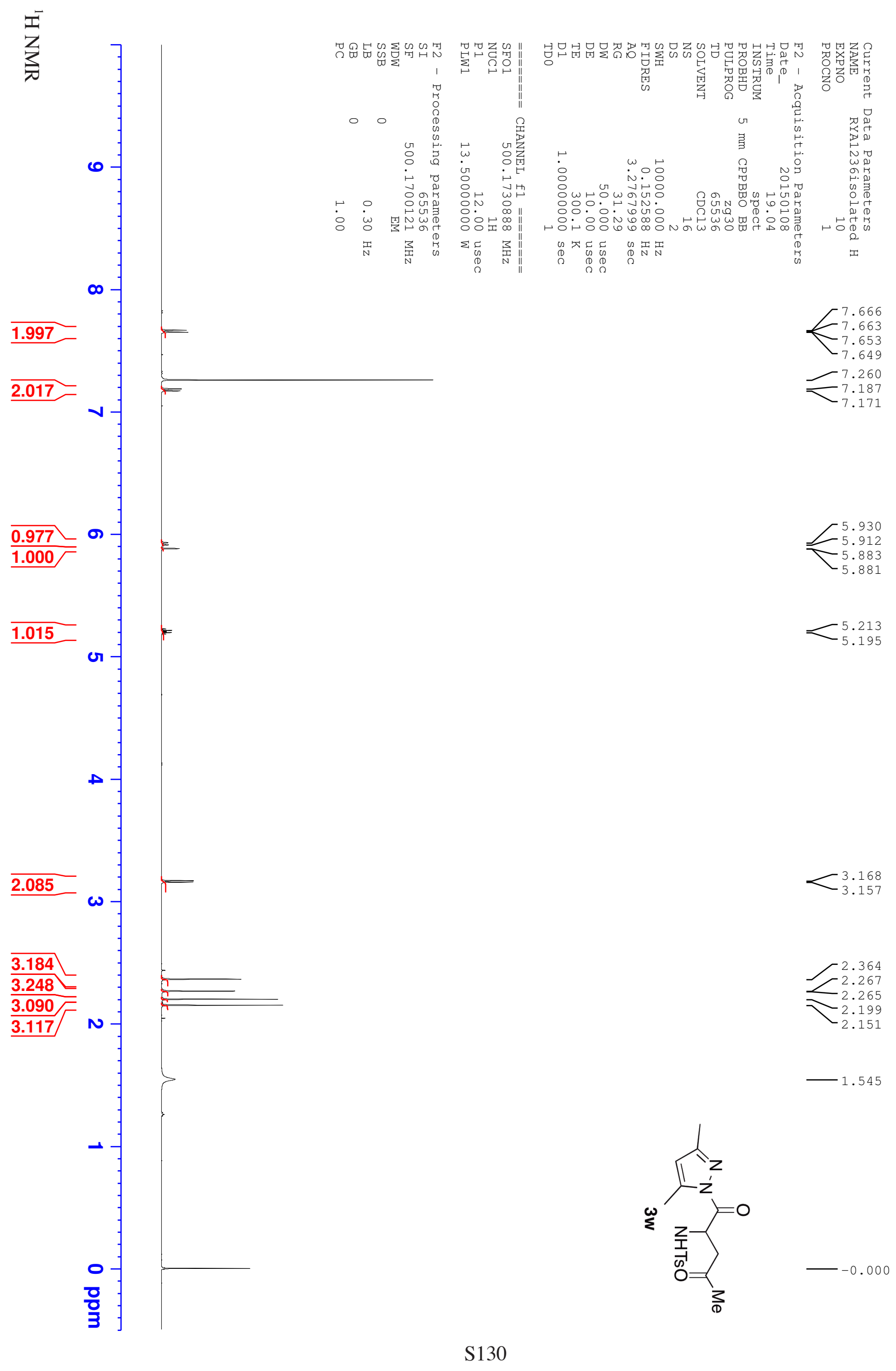


$\overbrace{}^{2}$

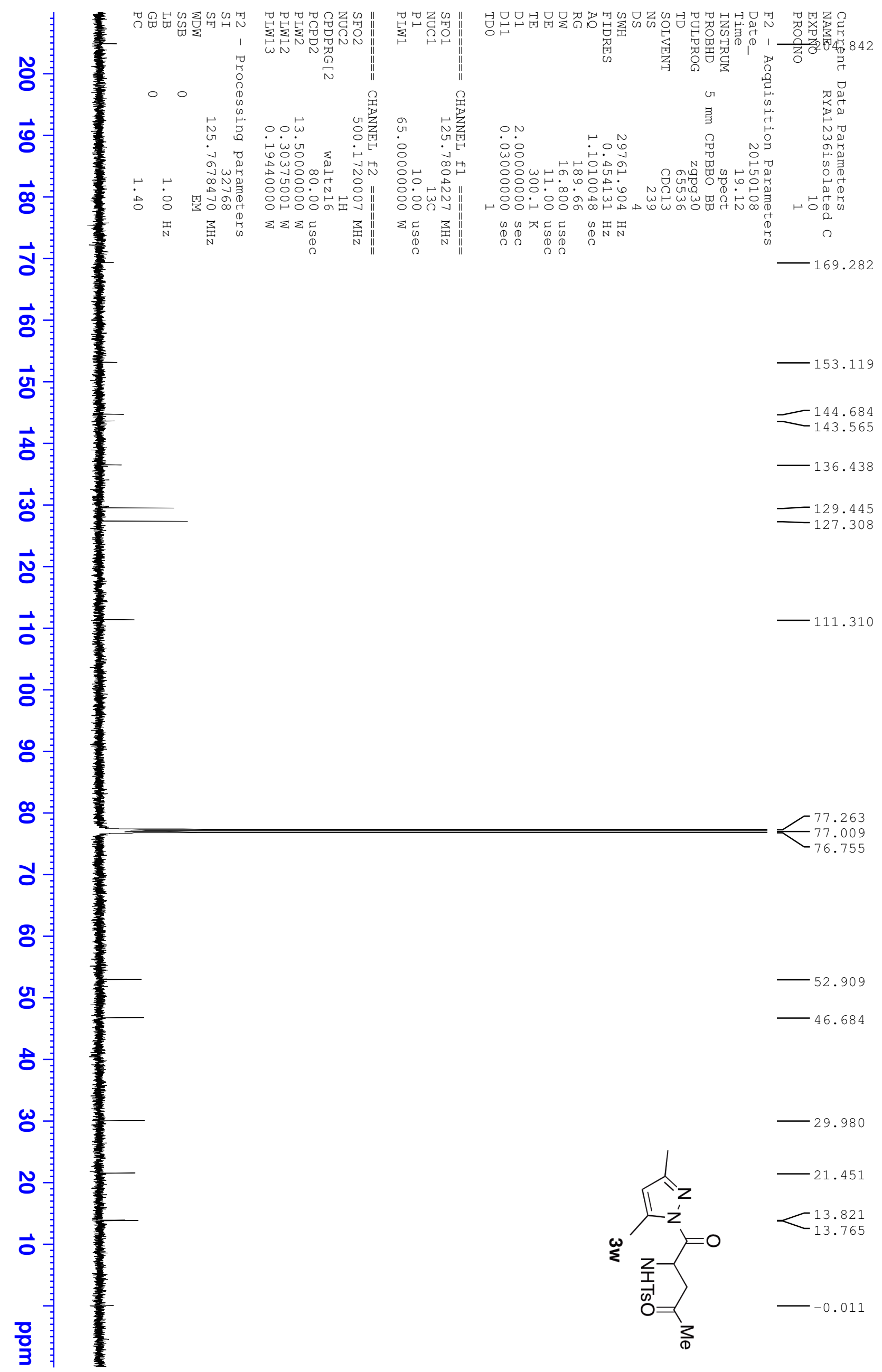



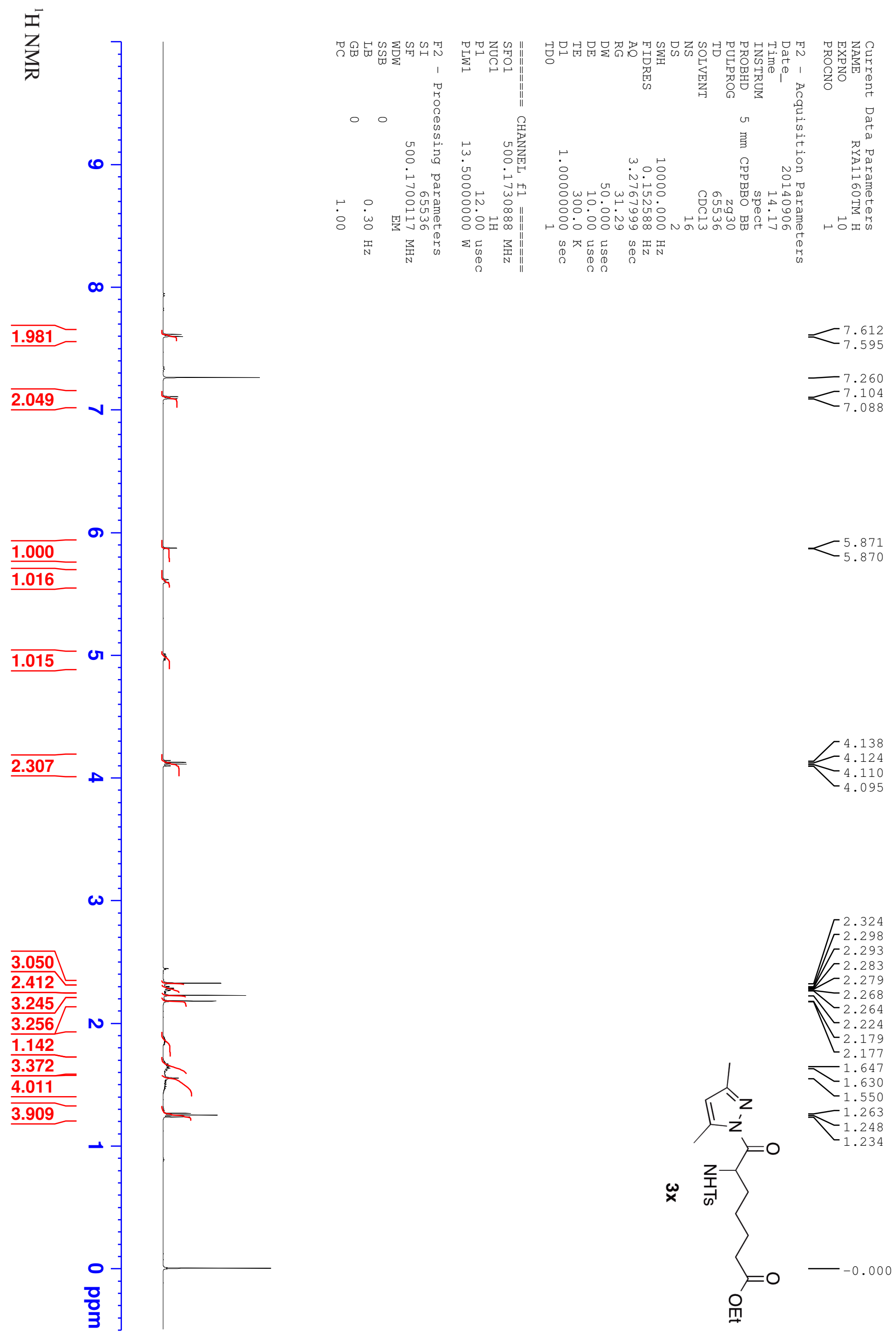
$\overbrace{0}^{\bar{\omega}}$

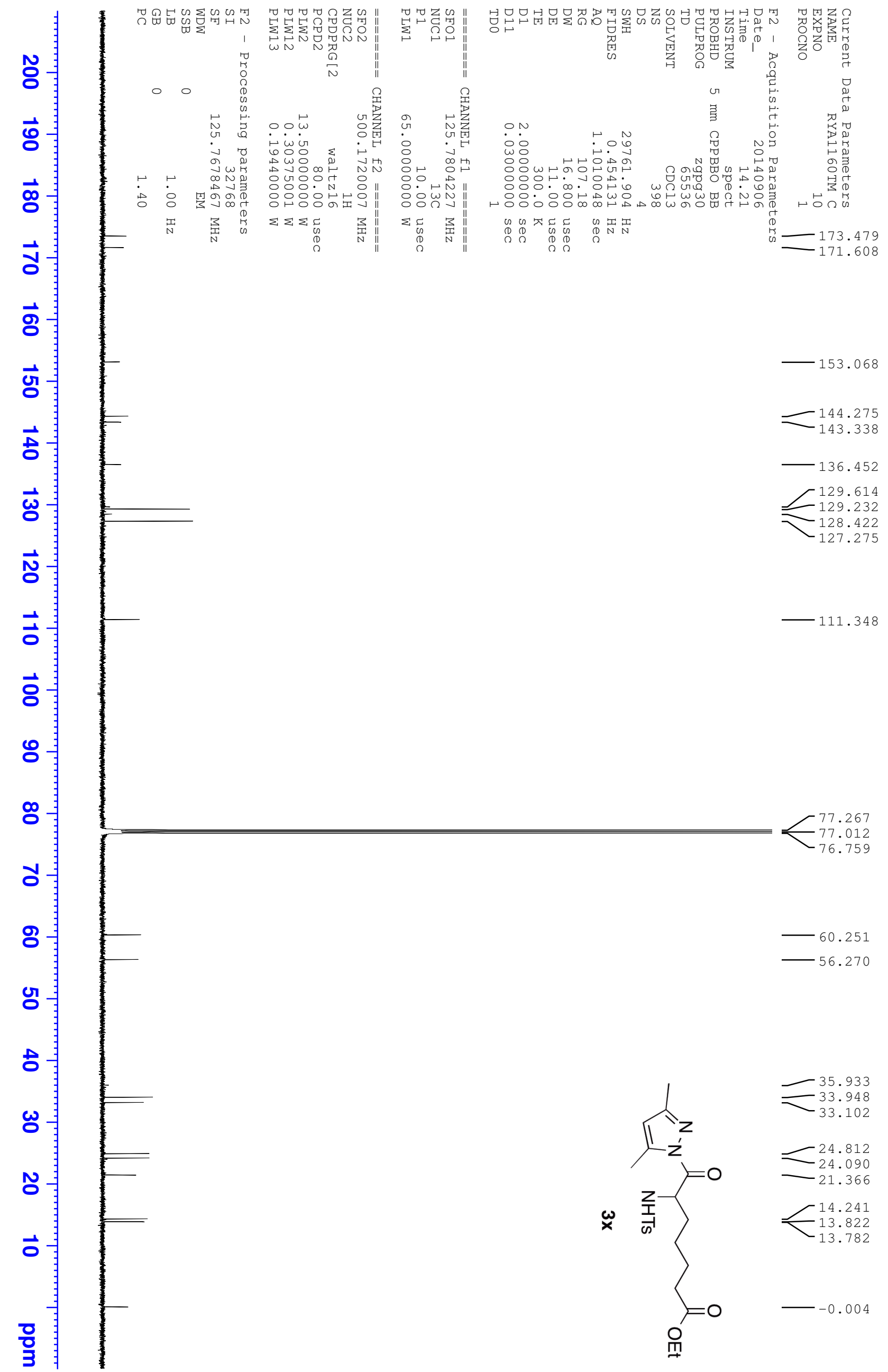




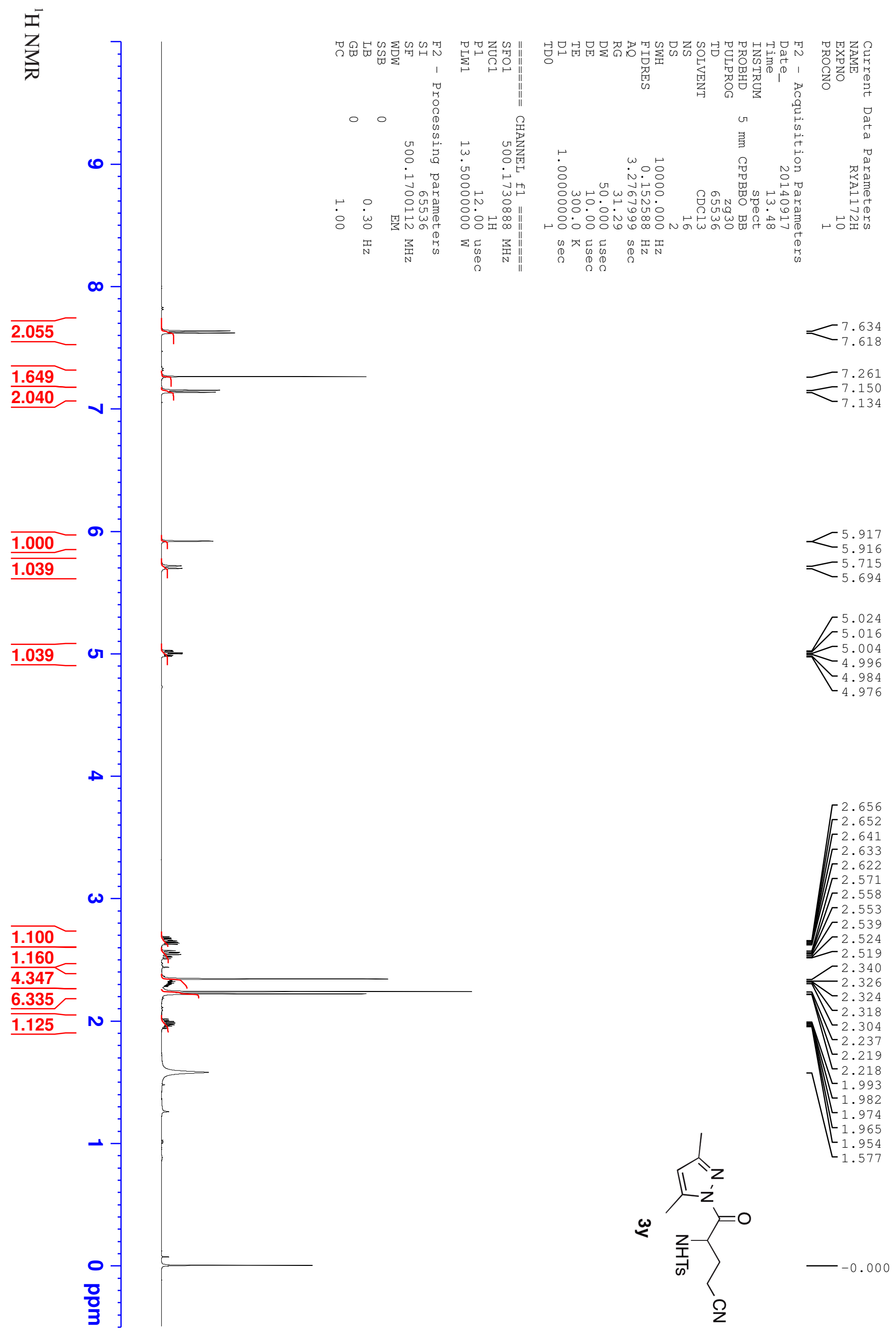


$\overbrace{}^{2}$

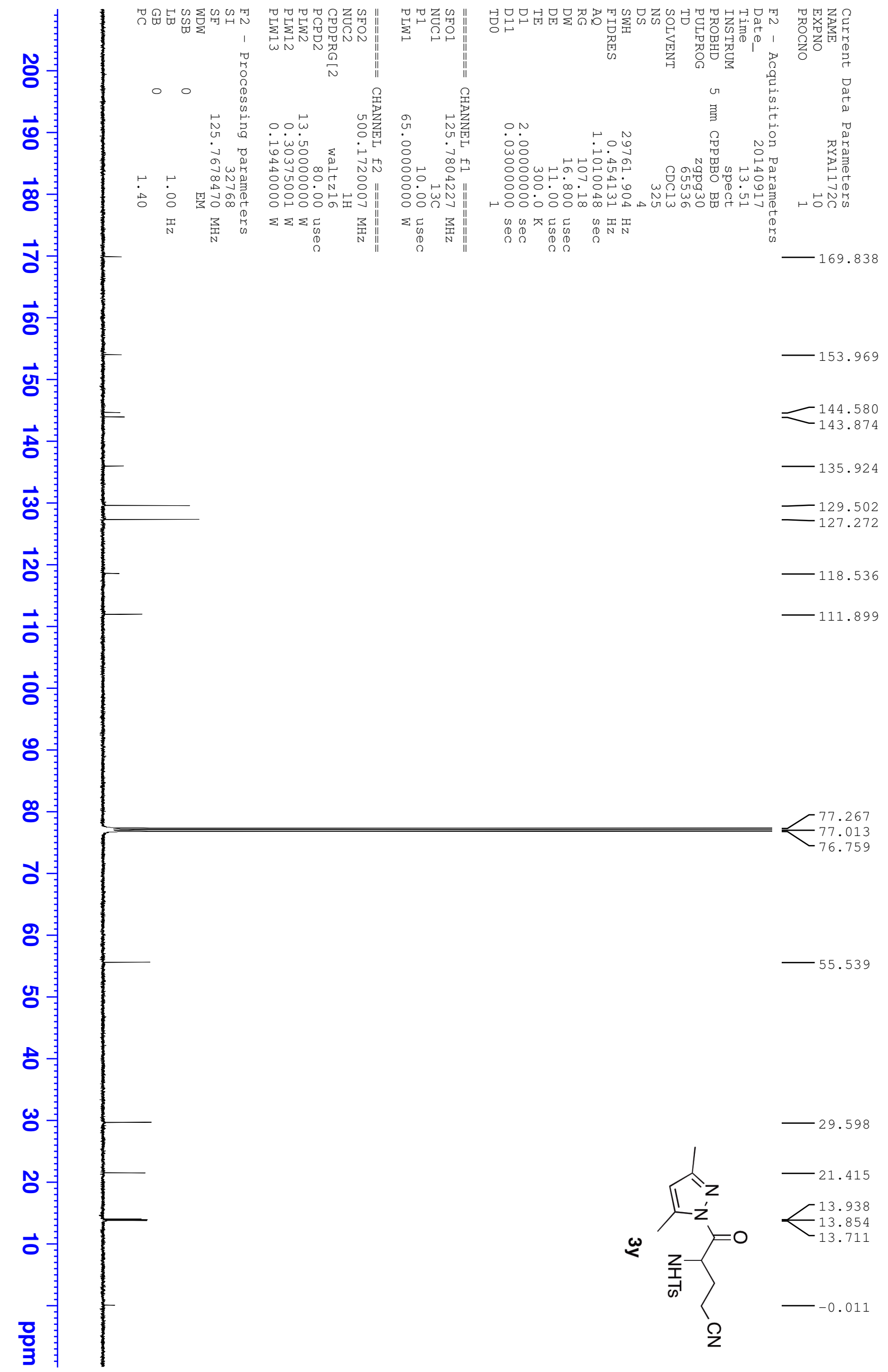




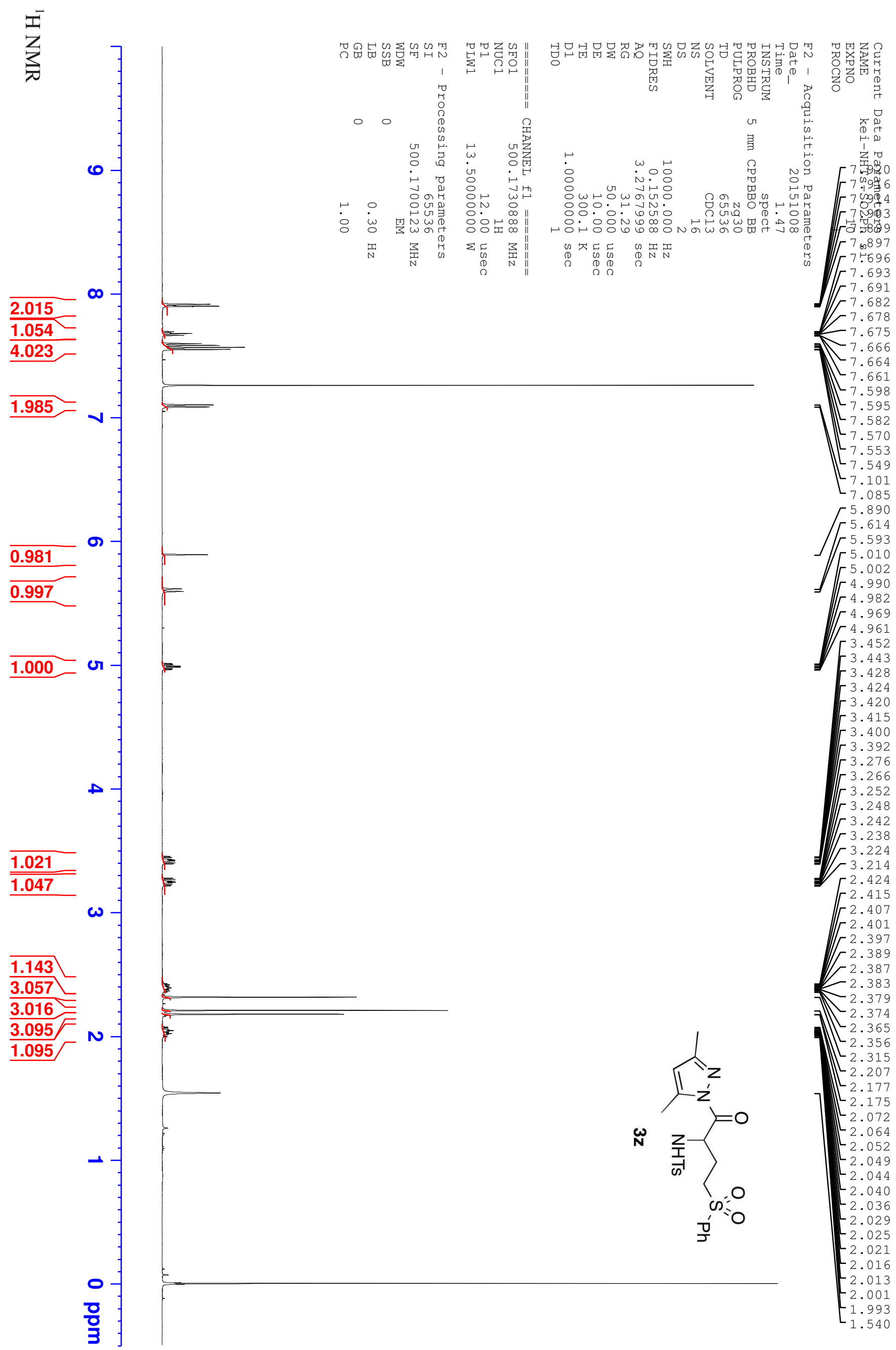


$\overbrace{}^{2}$

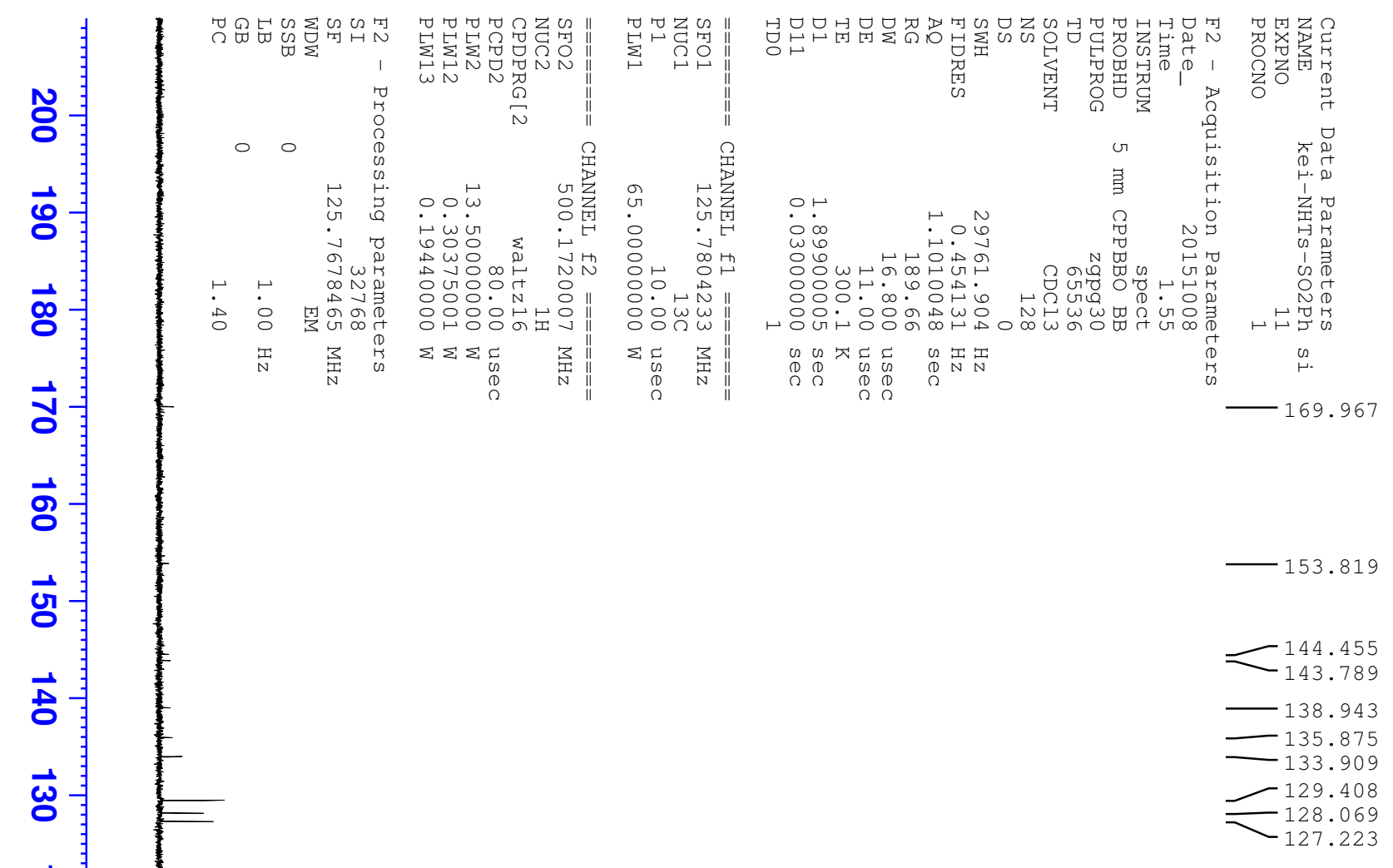

$\vec{\circlearrowleft}$

$\vec{\Delta}$

$\overrightarrow{8}$

8

ஃ

¿

8

잉

8

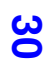

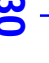

凊

$\overrightarrow{0}$

응 青

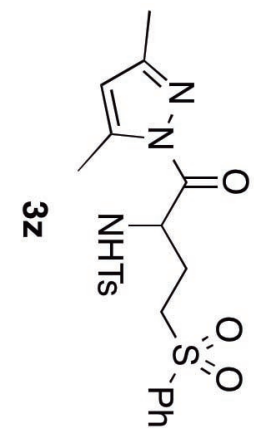

$-26.496$

$-21.389$

$\leftarrow 13.856$

$-13.682$ 


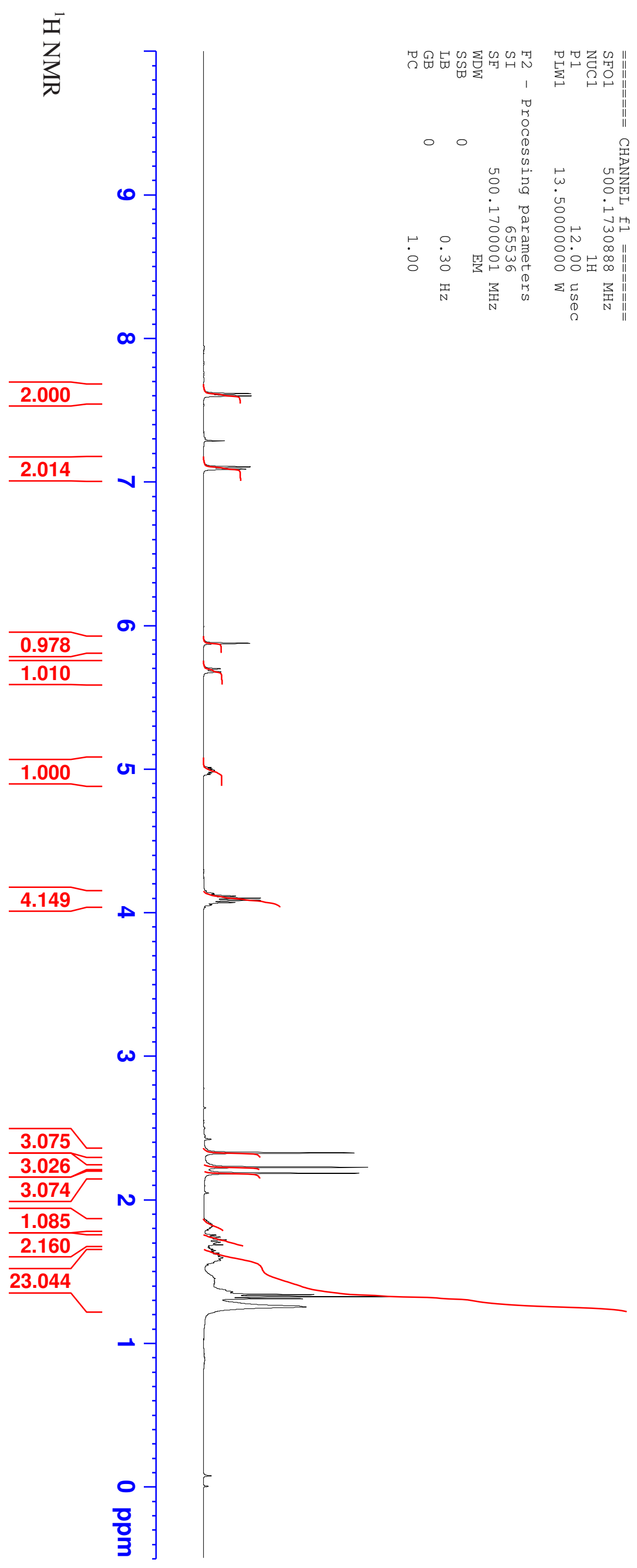

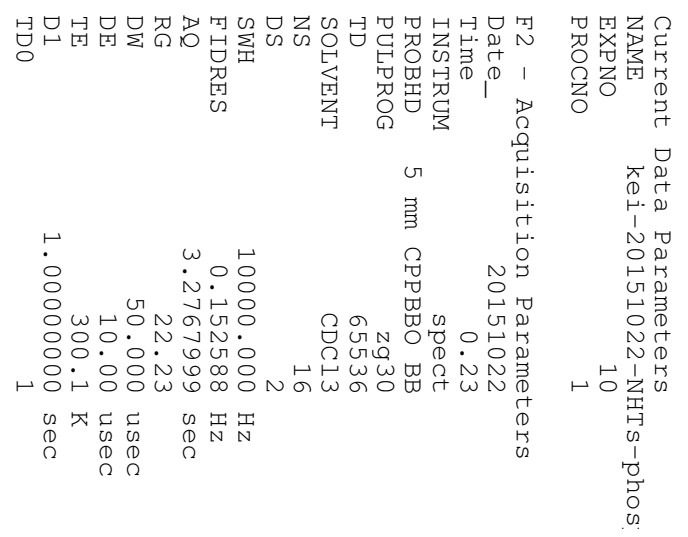

$-7.612$

7.596

7.102

7.086

$-5.873$

$\begin{array}{r}5.696 \\ \hline\end{array}$

5.674

4.999

4.989

4.981

4.112

4.097

4.083

4.068

4.068

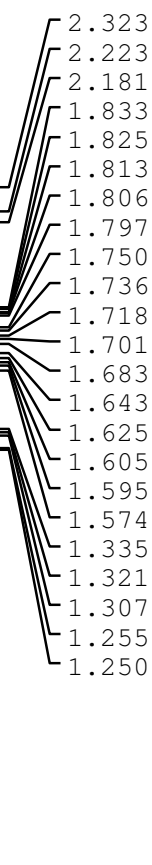

ón 
$\overbrace{}^{\bar{\omega}}$
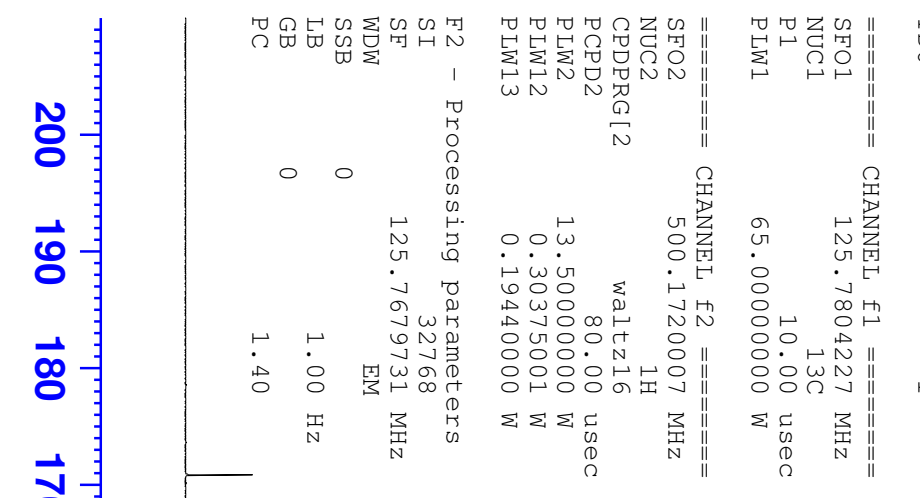

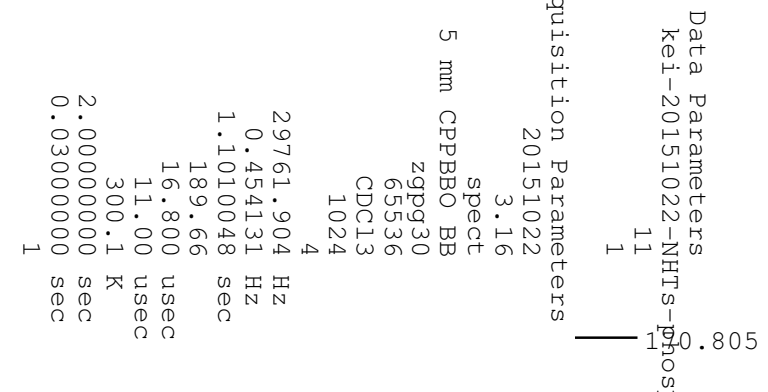

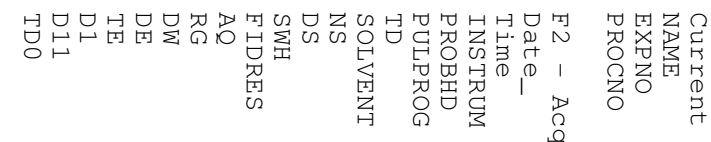

$\vec{\circ}$

ज्ञ

$\vec{f}$

$\bar{F}$

$-143.226$

$-142.236$

$-135.526$

$\vec{\omega}$

$+$

$-128.171$

26.235

$\overrightarrow{\text { N }}$

$\overrightarrow{\overrightarrow{0}}$

$\overrightarrow{8}$

8

ø

o

8

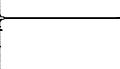

-60.399
-60.348

$-55.429$

응

to

๗

ก

o

응

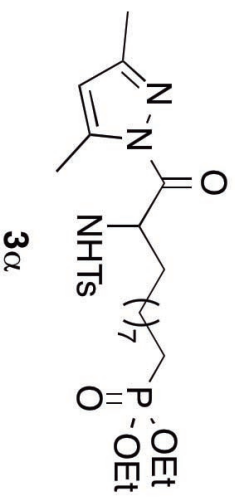

32.444
29.635

29.635
29.499

28.217
28.184

$r^{28.184}$

- 28.035

25.210
-24.198

24.198

724.095

-21.408
21.366

$\checkmark\lfloor-20.336$

$\left[\begin{array}{r}15.488 \\ 15.439\end{array}\right.$

12.810

12.787

임 


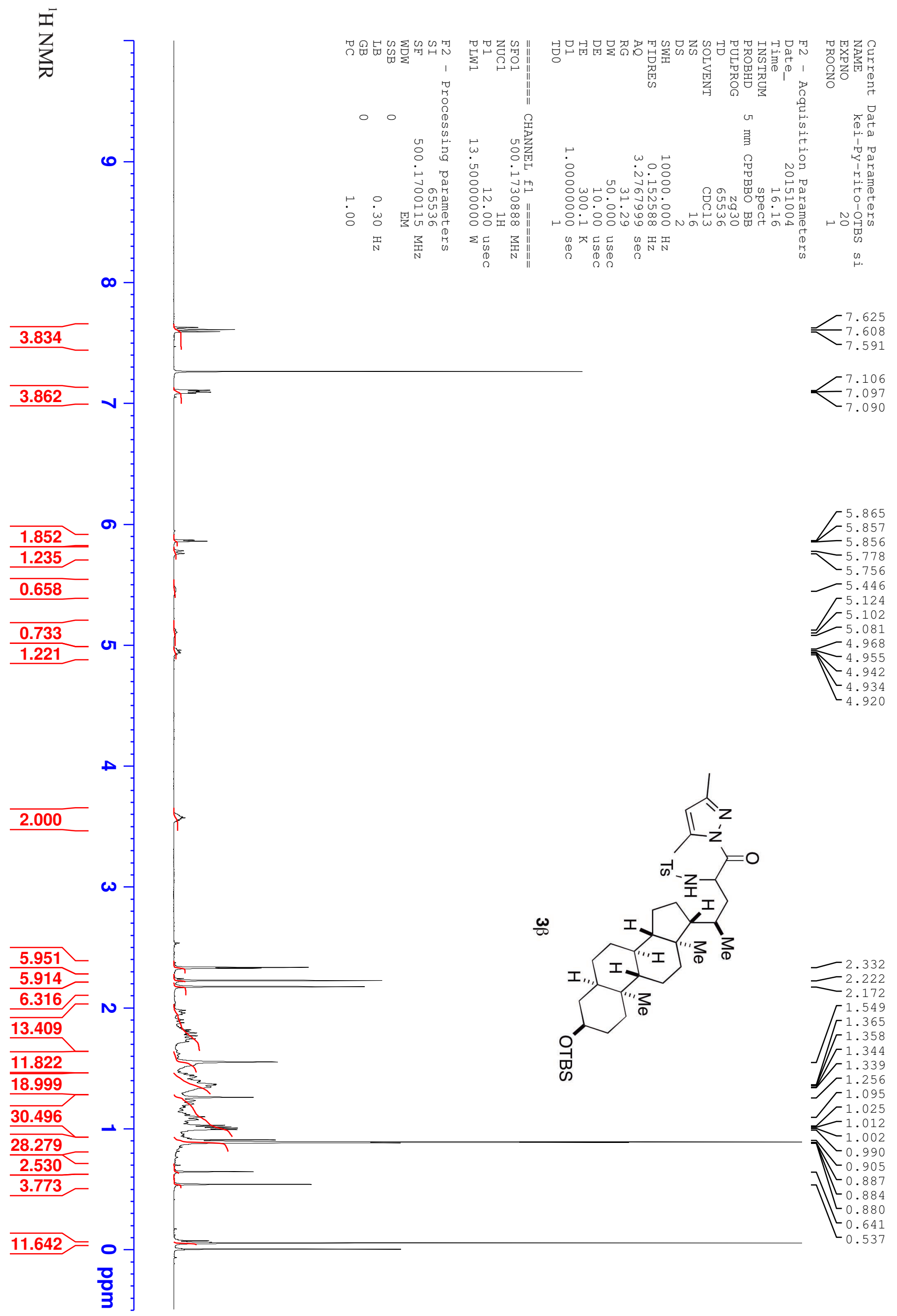


$\overbrace{}^{\bar{\omega}}$

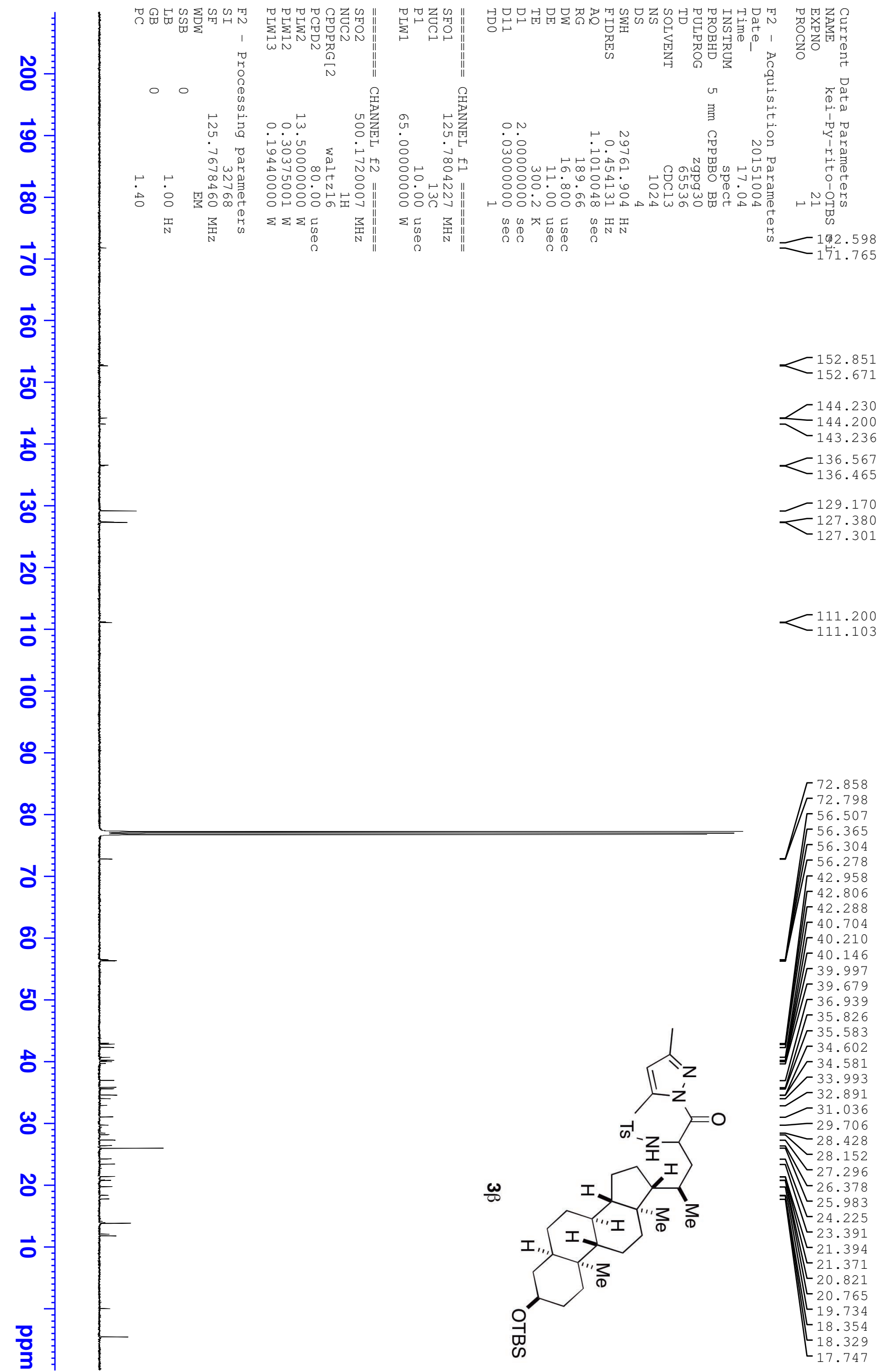




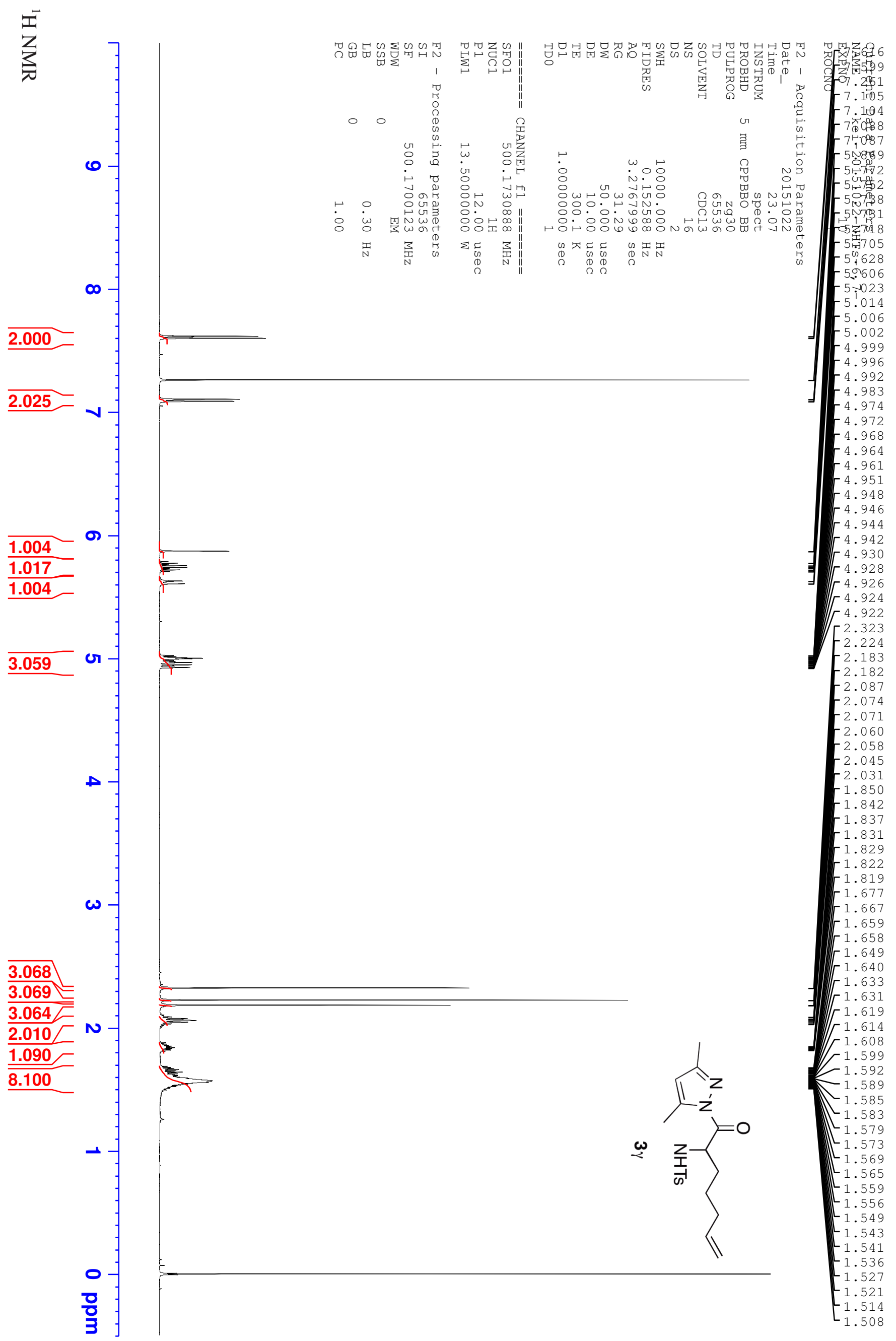


$\overbrace{}^{\bar{\omega}}$

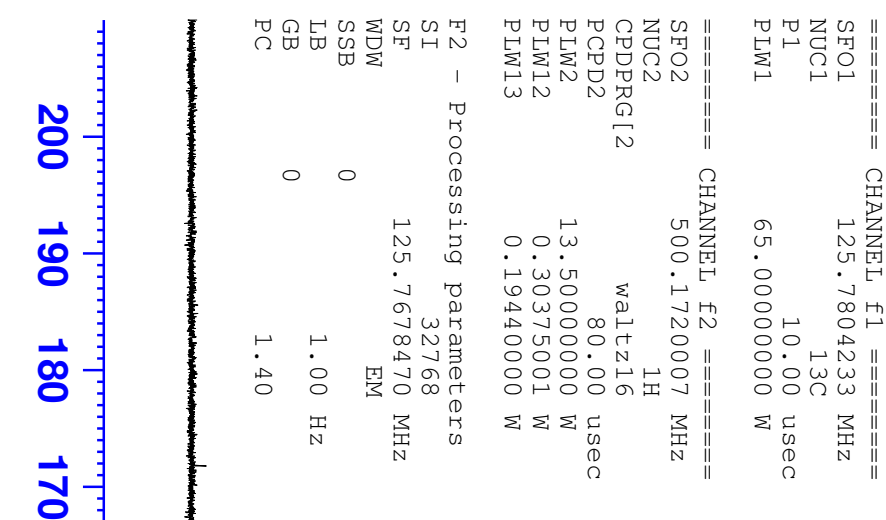

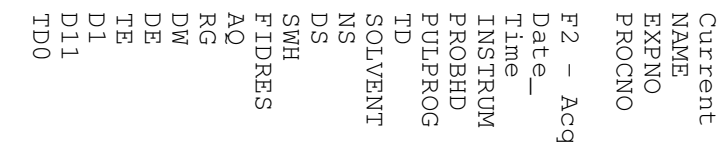

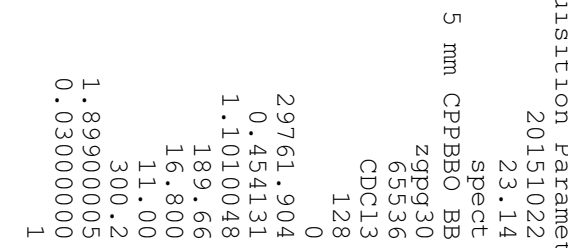

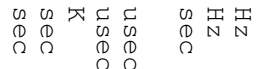

足

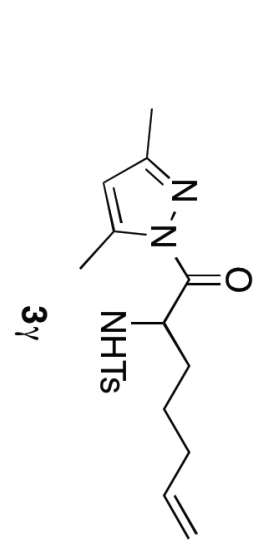

32.909
-32.817

$-24.531$ 